The copyright of this thesis vests in the author. No quotation from it or information derived from it is to be published without full acknowledgement of the source. The thesis is to be used for private study or noncommercial research purposes only.

Published by the University of Cape Town (UCT) in terms of the non-exclusive license granted to UCT by the author. 


\section{Development of a System for Testing Grid-Connected Permanent Magnet Wind Generators}

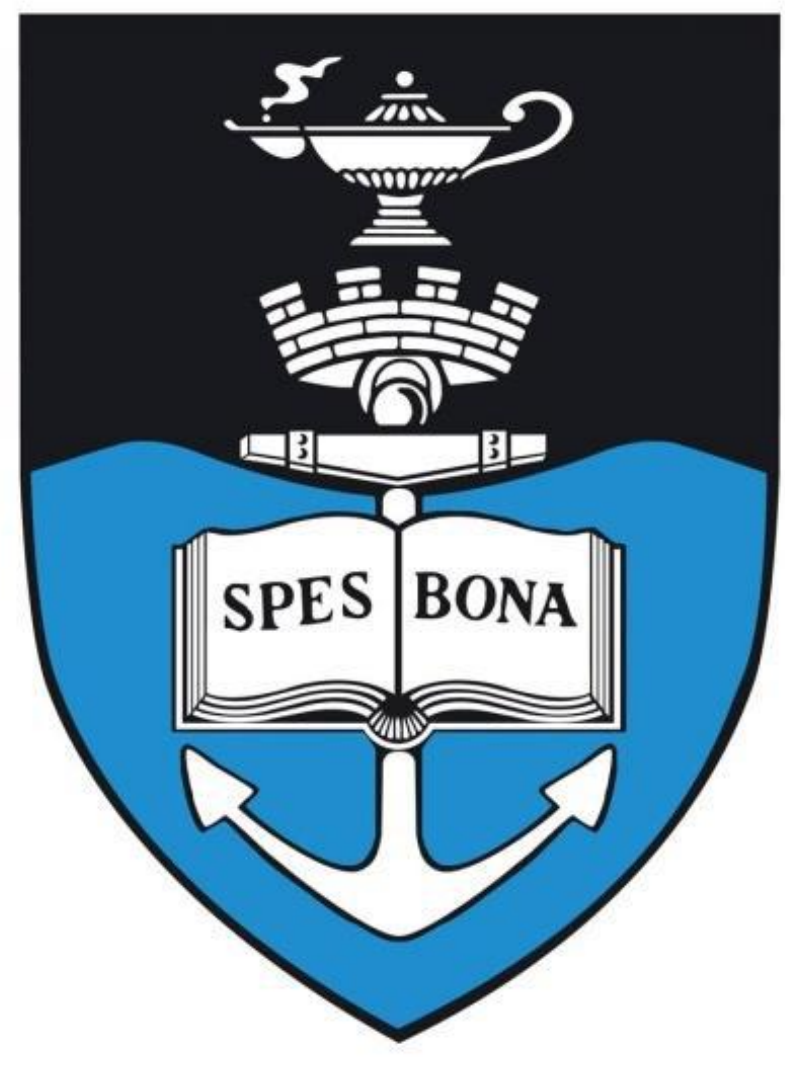

\section{By: Jacques Gerard de la Bat}

Thesis submitted to the Department of Electrical Engineering, University of Cape Town, in complete fulfilment of the requirements for the degree of Master of Science in Electrical

Engineering

28th of October 2011 


\section{Declaration}

This dissertation is submitted to the Department of Electrical Engineering, University of Cape Town, in complete fulfilment of the requirements for the degree of Master of Science in Electrical Engineering. It has not been submitted before for any degree or examination at this or any other university.

"I know the meaning of plagiarism and declare that all the work in the document, save for that which is properly acknowledged, is my own."

J.G. de la Bat

28th of October 2011 


\section{Acknowledgments}

I would like to thank my supervisors Dr. M.A. Khan and Dr. P. Barendse for their guidance and understanding throughout the last two years.

Special thanks go to Chris Wozniak and Phillip Titus, who were patient and helpful when things did not go as planned with the experimental setup. Without them I would not have been able to complete this work.

To my fellow members of the AMES group, especially Hartmut Jagau, Jonothan Nye, Dr Richard Okou, Chris de Beer, Direshni Reddy and Henry Liu for all the time that was set aside to help me.

To my friends and family, thank you for your support. I am looking forward to a bright future with all of you. 


\section{Abstract}

Renewable energy will be included in the South African Energy Mix over the next two decades. The introduction of renewable energy will reduce South Africa's carbon emissions and also stimulate the economy through job creation as well as creating a local manufacturing sector. South Africa has a large coastal region which is ideal for wind energy deployment. The integration of wind power into the grid needs to be understood as well as the possible problems associated with it. The objective this thesis is to develop a laboratorybased system which can serve as a tool for studying non-ideal conditions associated with the integration of grid-connected Permanent Magnet (PM) wind generators. In particular, non-ideal conditions associated with the grid in the form of unbalanced supply voltages or the turbine in the form of turbulence and other effects.

The main emphasis of the work presented in this thesis was to develop a thorough understanding and implementation of a grid-connected PM Wind Generator system. A turbine emulator was developed using an 8-pole induction machine, which was coupled directly to the PM wind generator. Turbine effects that were investigated include: wind shear and tower shadow. Wind turbulence was also implemented on the turbine emulator.

The PM generator was connected to the grid through a back-to-back converter and a $\mathrm{LCL}$ filter. A step-by-step approach was used for the filter design. Both sides of the converter were controlled using vector control. A control systems analysis approach was used to tune the controller parameters.

All system components were simulated and the results were verified through experimental testing. The system performance was tested with all the turbine effects as well as turbulence. Further tests were conducted on the effect of unbalanced supply voltage conditions using standard vector control as well as dual vector current control.

It was found that that the dual vector control technique produced balanced currents under grid conditions that resembled a Type B voltage dip. Furthermore, it was found that maximum power point tracking was successfully implemented and the system was found to be stable and operate at an efficiency of $80 \%$ during turbulent conditions. 


\section{Table of Contents}

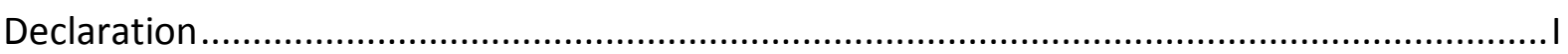

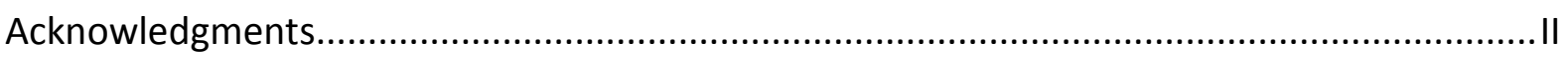

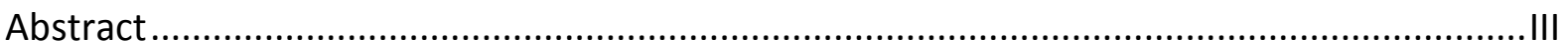

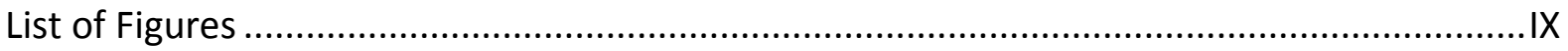

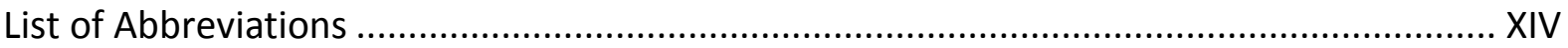

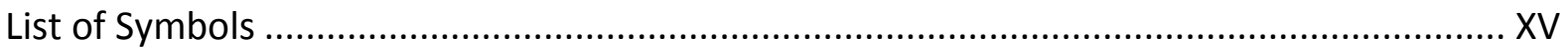

INTRODUCTION

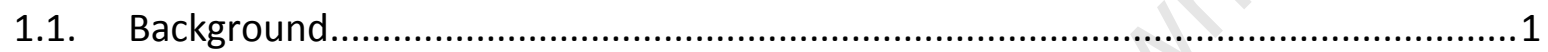

1.1.1. Electricity Generation in South Africa......................................................

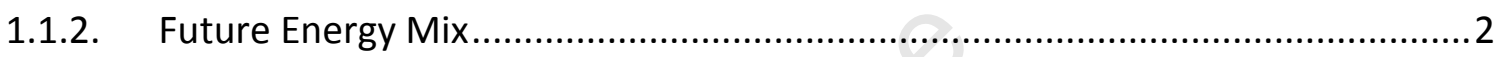

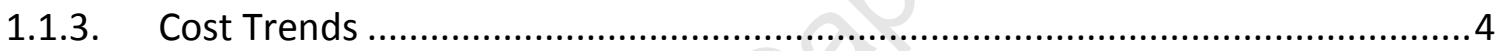

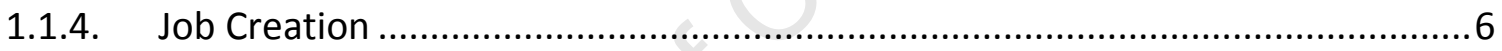

1.1.5. Near Term Grid Integration Issues............................................................

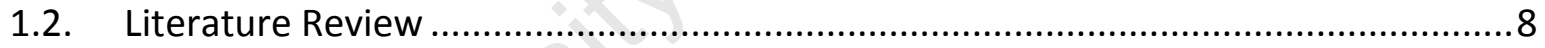

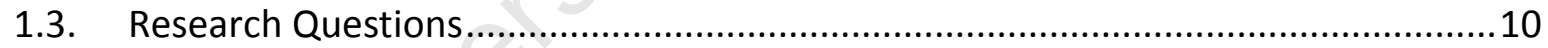

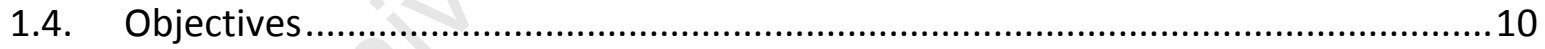

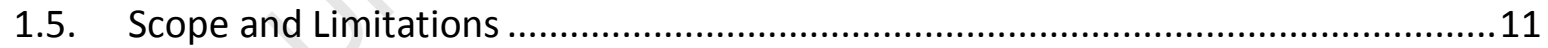

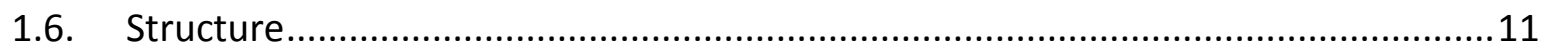

WIND ENERGY CONVERSION SYSTEM THEORY DEVELOPMENT .......................................12

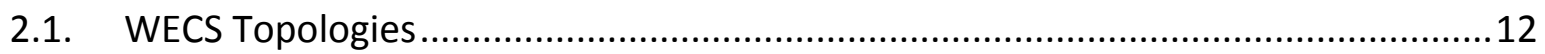

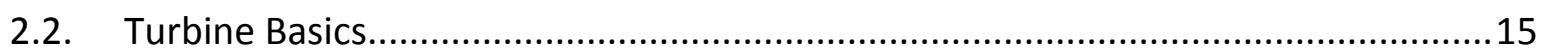

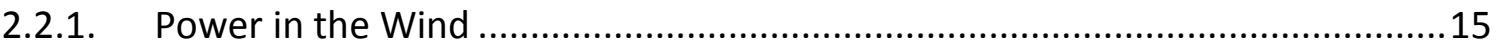

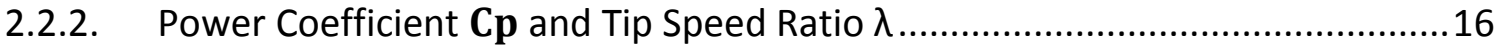

2.2.3. Maximum Power Point Tracking (MPPT) .................................................. 17

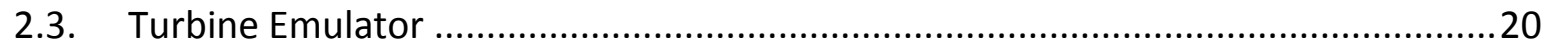




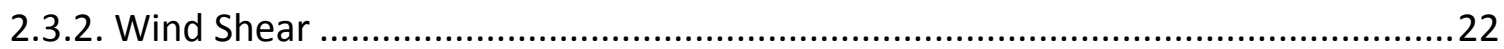

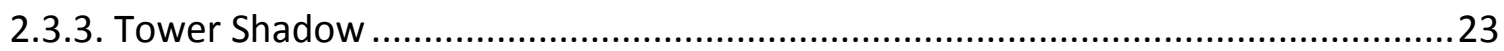

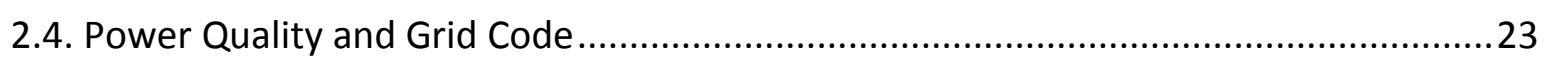

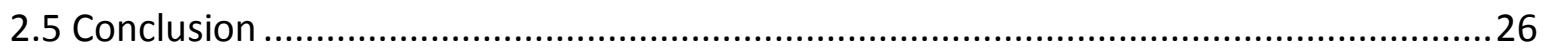

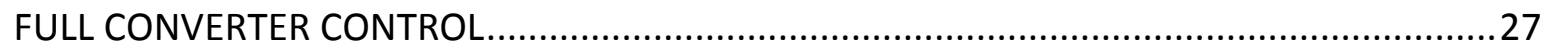

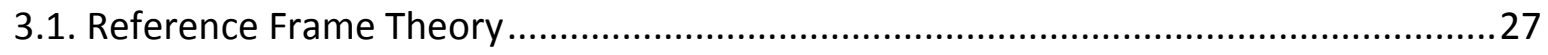

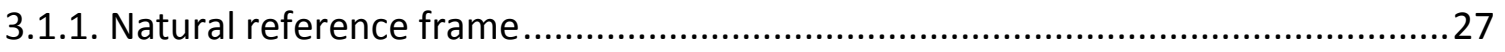

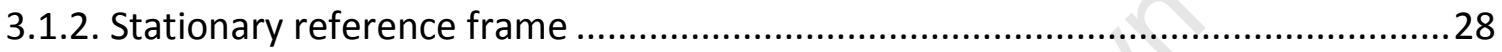

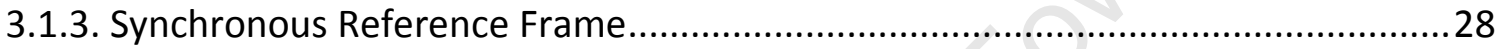

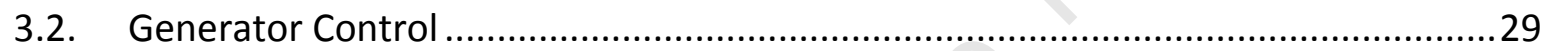

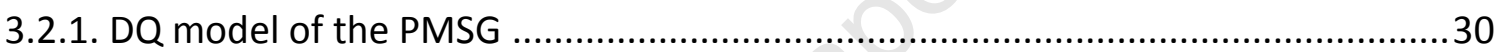

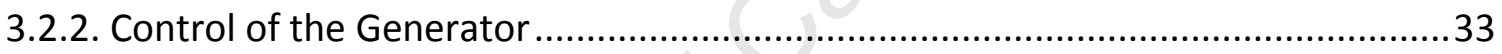

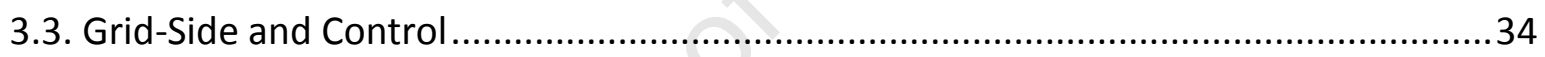

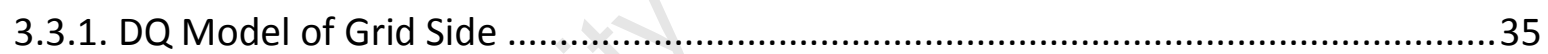

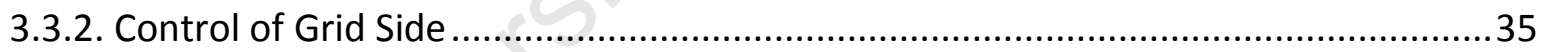

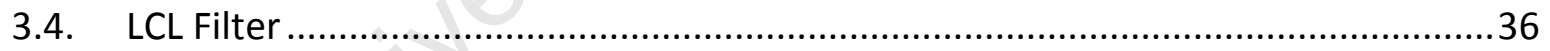

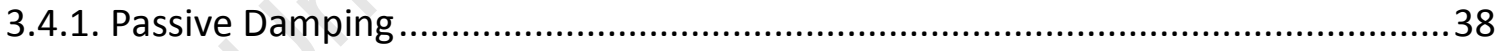

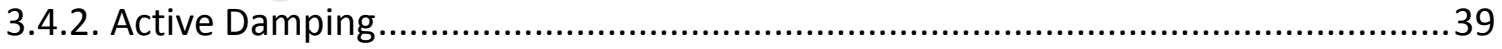

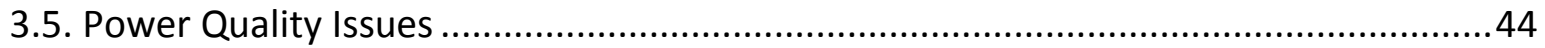

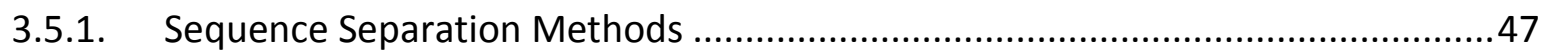

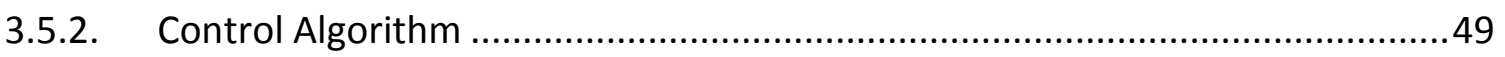

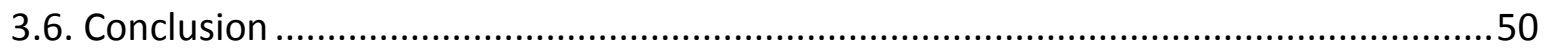

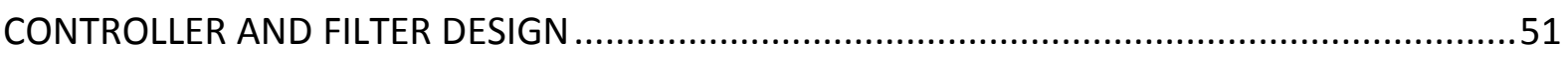

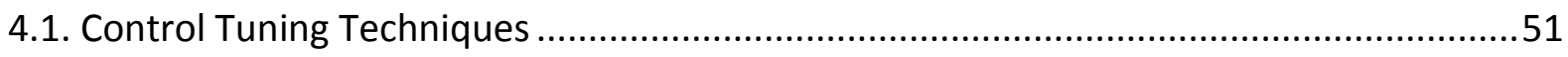

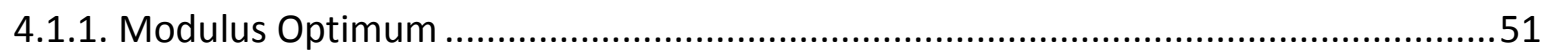




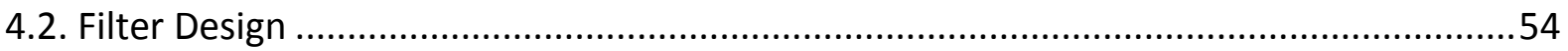

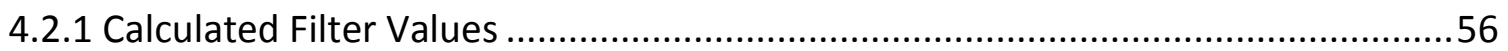

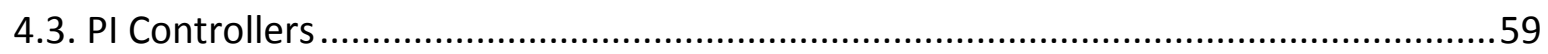

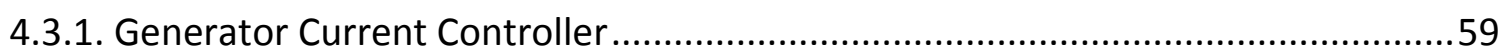

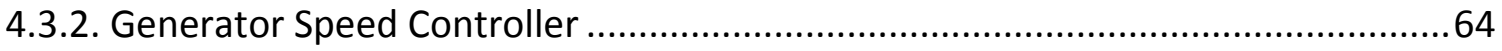

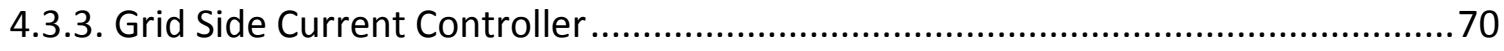

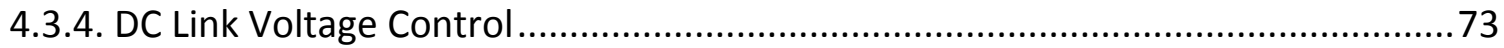

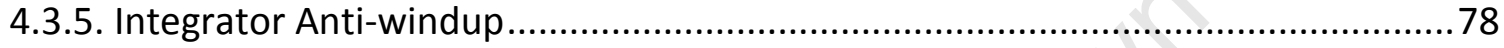

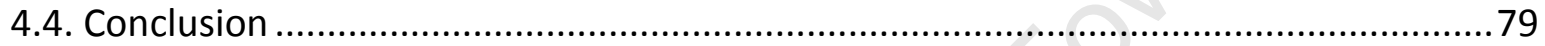

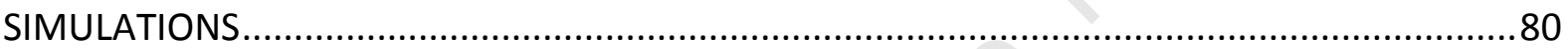

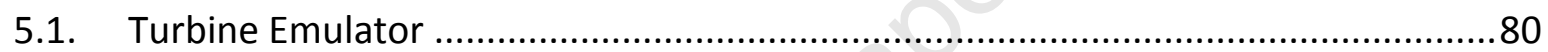

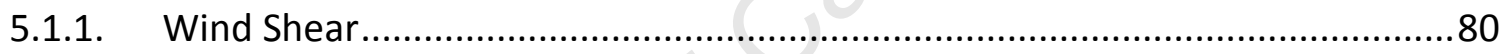

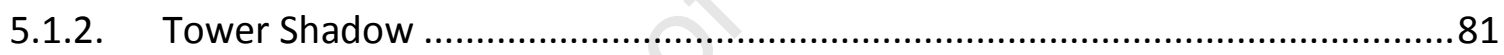

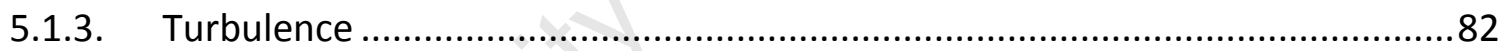

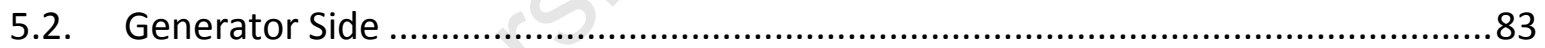

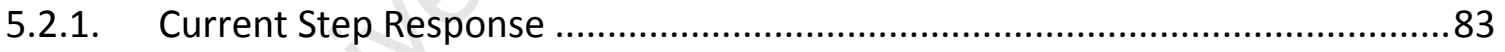

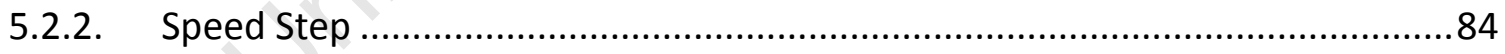

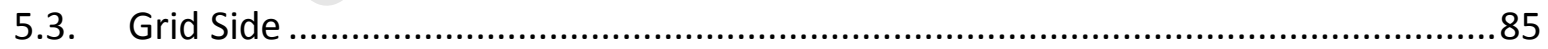

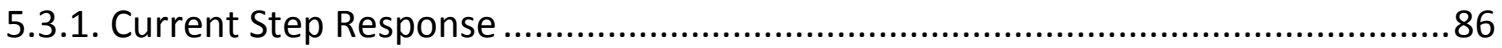

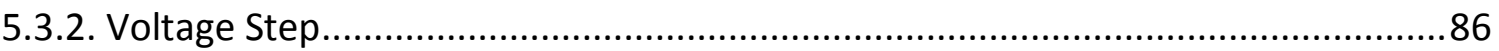

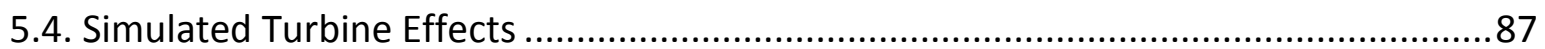

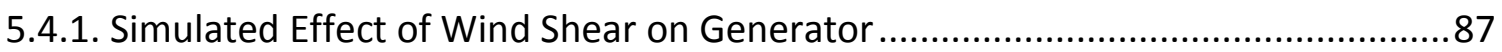

5.4.2. Simulated Effect of Tower Shadow on Generator .................................................... 88

5.4.3. Simulated Effect on Generator with all Turbine Effects......................................89

5.5. Control Effectiveness Under Unbalanced Operating Conditions...........................90 


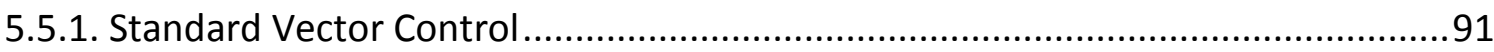

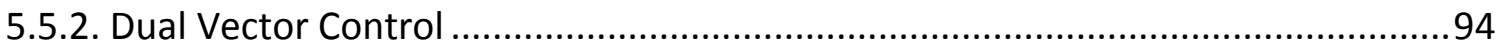

5.6. Simulated Machine MPPT Under Turbulent Conditions .............................................98

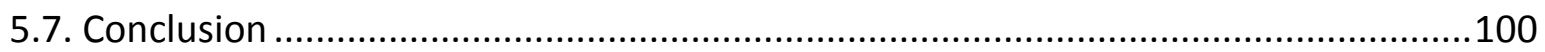

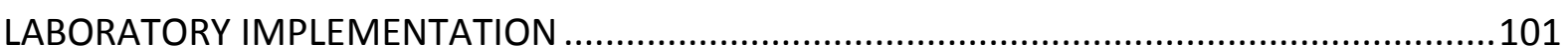

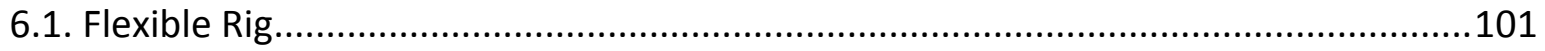

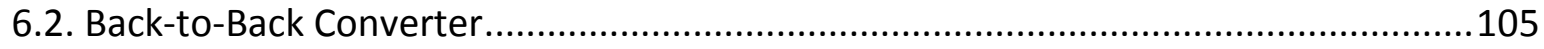

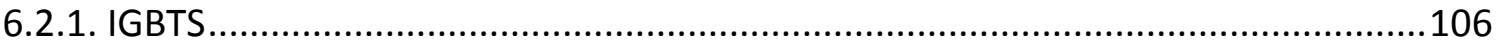

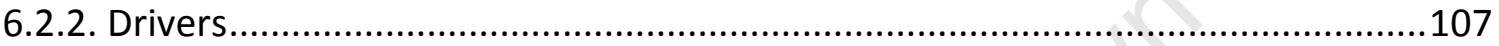

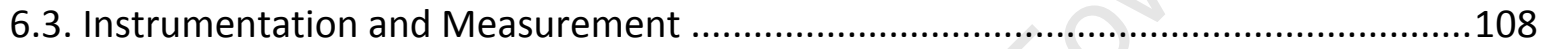

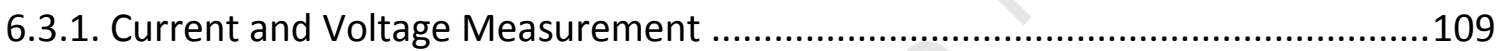

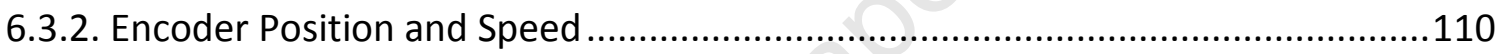

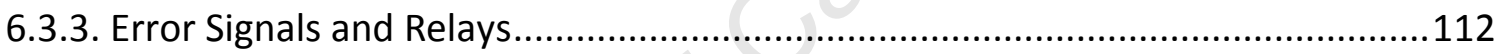

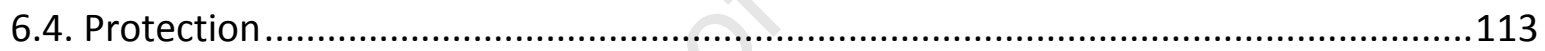

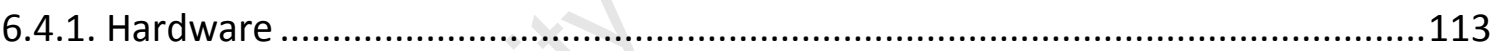

6.4.2. Software

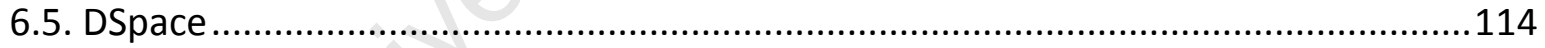

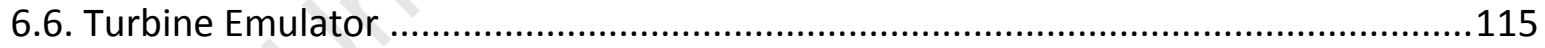

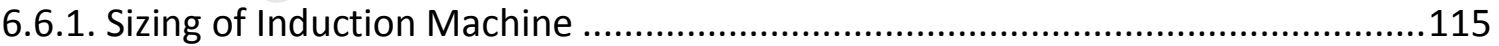

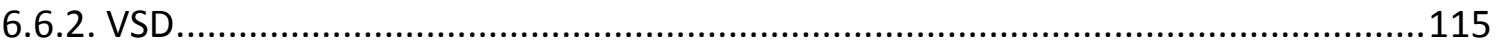

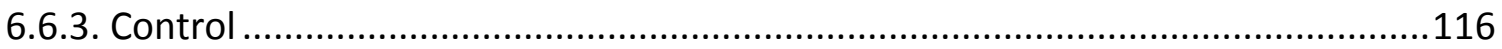

6.7. Implementation of Power Quality Effects.............................................................116

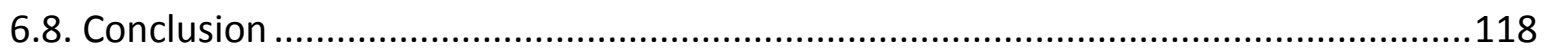

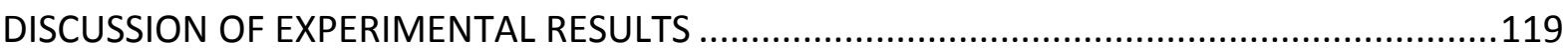

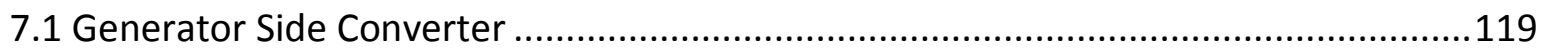

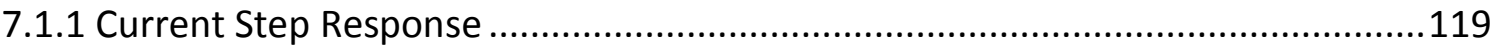


7.1.2. Speed Step Response .120

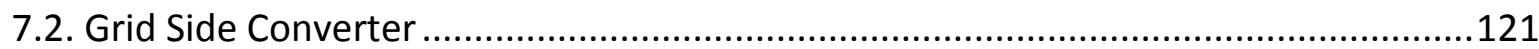

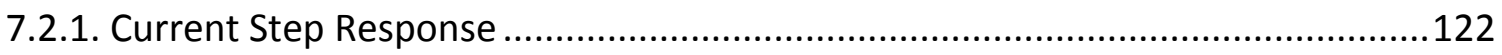

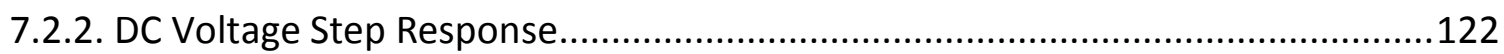

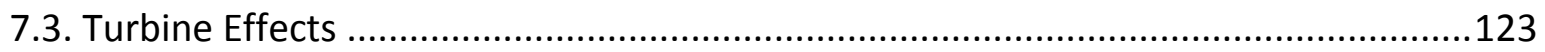

7.3.1. Experimental Effects of Wind Shear on the Generator....................................124

7.3.2. Experimental Effects of Tower Shadow on the Generator ................................125

7.3.3. Experimental Effect on Generator with All Turbine Effects ..............................126

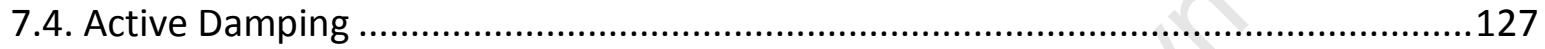

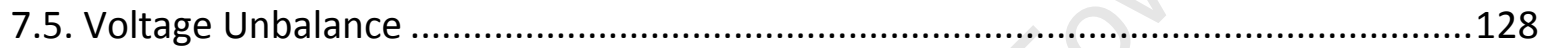

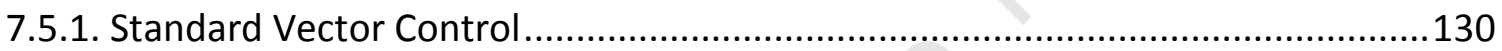

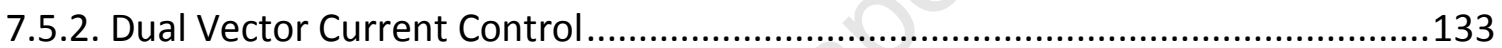

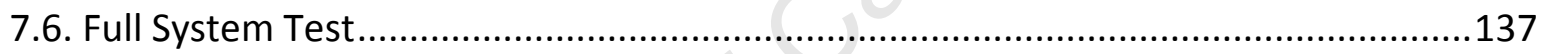

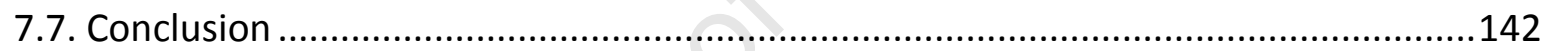

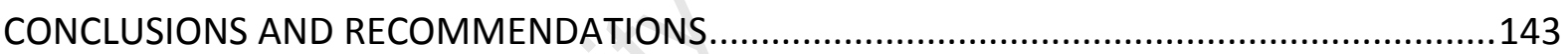

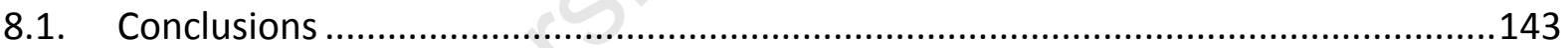

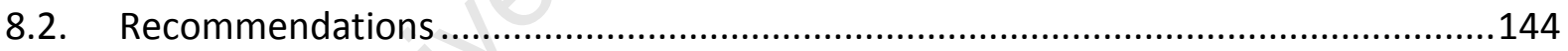

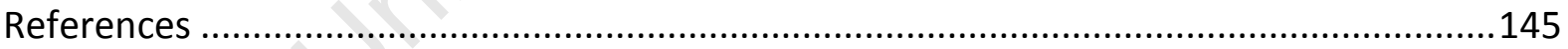

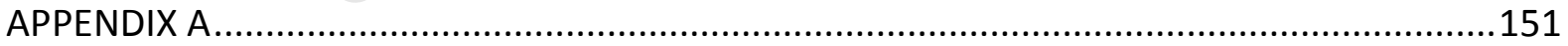

Procedure 1:

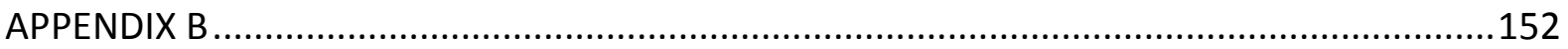




\section{List of Figures}

Figure 1: Million ton of CO2 emission from the burning of fossil fuels in 2006 [4] ................2

Figure 2: Average annual wind speeds at $10 \mathrm{~m}$ above ground in $\mathrm{ms}-1$ [7] ........................... 4

Figure 3: Jobs by Technology and Type 2010, 2020 and 2030 [11] .......................................

Figure 4: Induction Generator with a Back-to-Back Converter Topology ..............................12

Figure 5: Doubly-Fed Induction Generator with a Back-to-Back Converter Topology............13

Figure 6: Permanent Magnet Synchronous Machine with a Diode Rectifier-VSI Converter

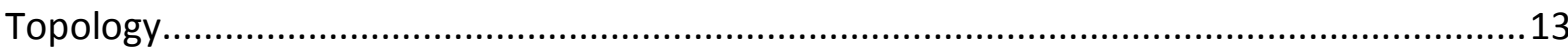

Figure 7: Permanent Magnet Synchronous Machine with a Diode Rectifier-Chopper-VSI Converter Topology 14

Figure 8: Permanent Magnet Synchronous Machine with a Back-to-Back Converter Topology .14

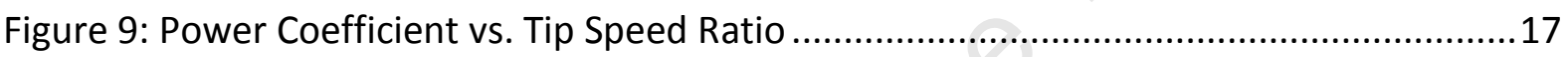

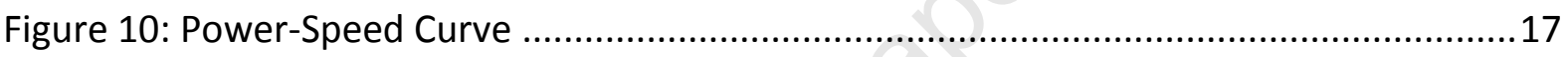

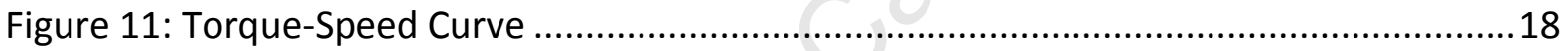

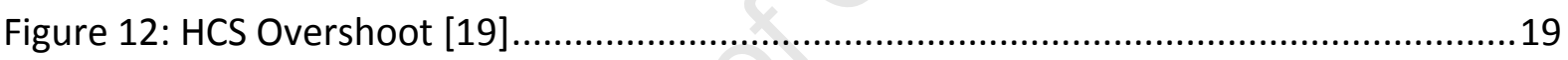

Figure 13: Direction Error for a Change in Wind Speed [19] ..............................................20

Figure 14: Requirements for Reactive Power Support During Voltage Drops [24] ................24

Figure 15: Low Voltage Ride Through Capability for a Wind Turbine [24] .............................24

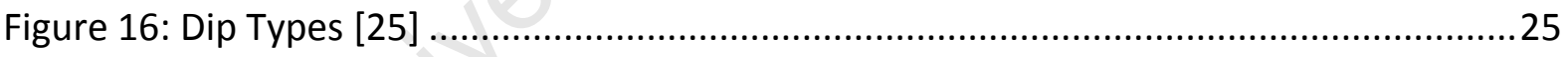

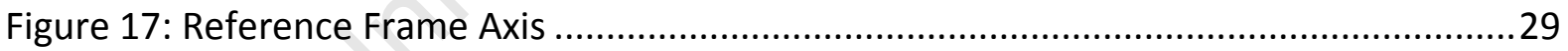

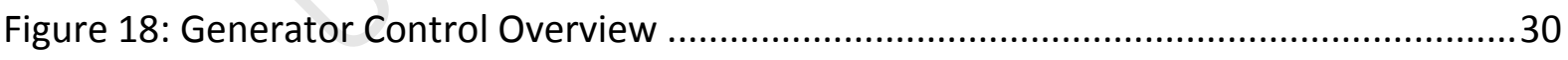

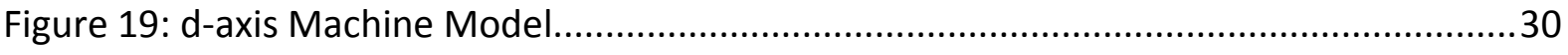

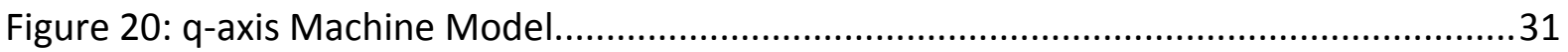

Figure 21: Detailed Generator Control Algorithm with MTA implemented..........................33

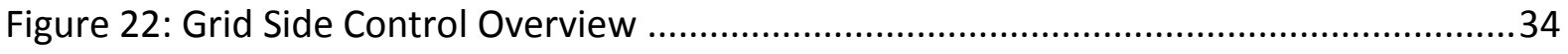

Figure 23: Detailed Control Algorithm for VOC with UPF control ........................................36

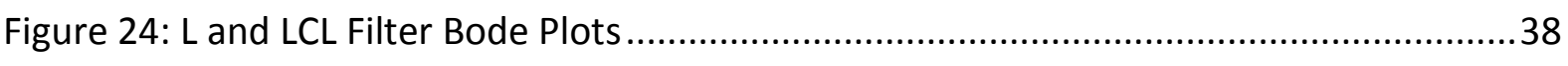

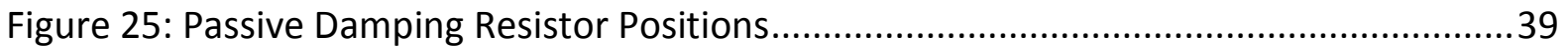

Figure 26: Block Diagram Implementation of Series Virtual Resistor [32] ............................40

Figure 27: Block Diagram Implementation of Parallel Virtual Resistor [32] .........................40 
Figure 28: Implementation of Lead-Lag Active Damping

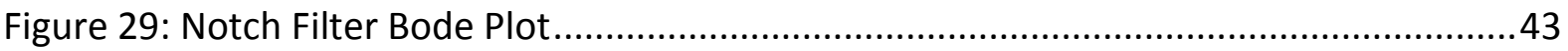

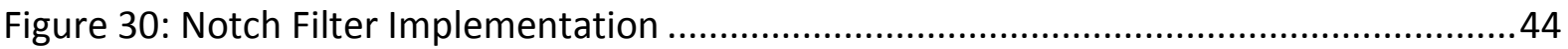

Figure 31: Comparison of Vd for Balanced and Unbalanced Voltages ..................................45

Figure 32: Positive and Negative Sequence Components of Unbalanced Three-Phase

Currents .46

Figure 33: Effect of Voltage Unbalance in the Stationary Reference Frame ..........................46

Figure 34:Filter Sequence Separation Method ................................................................. 48

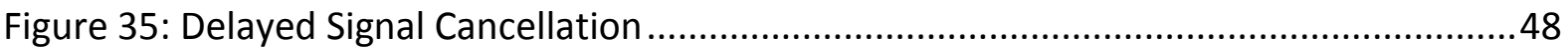

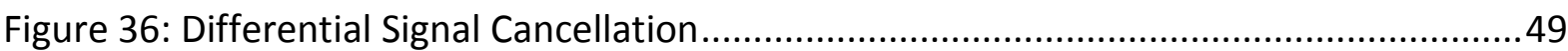

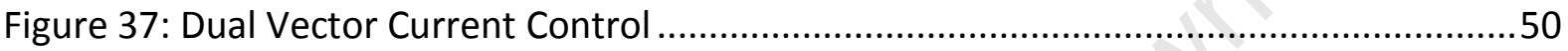

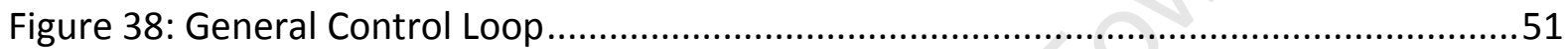

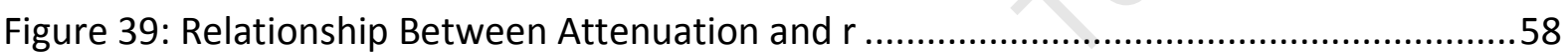

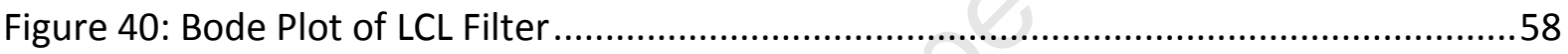

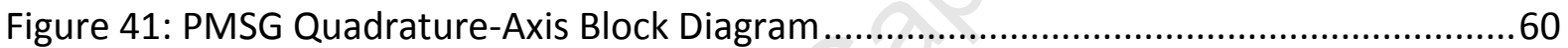

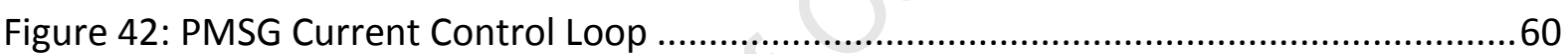

Figure 43: PMSG Current Control Loop Root Locus.............................................................63

Figure 44: PMSG Current Control Loop Bode Plot ..................................................................64

Figure 45: Comparison of Step Response between Current Loop and Approximation ..........65

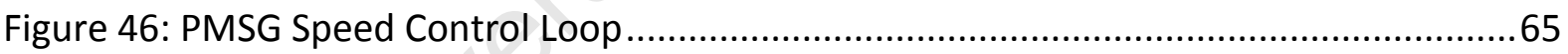

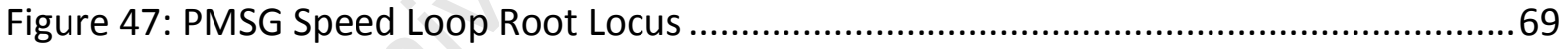

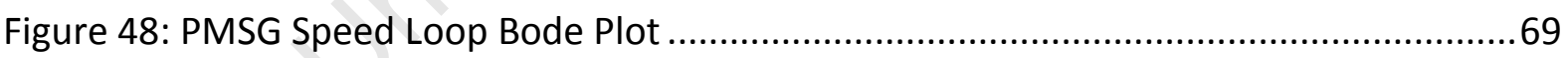

Figure 49: Grid Current Loop Block Diagram .................................................................... 70

Figure 50: Grid Current Loop Root Locus............................................................................ 72

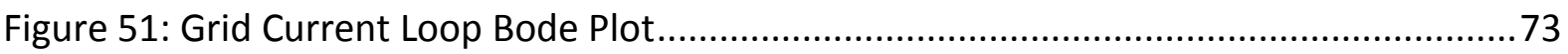

Figure 52: DC Link Voltage Loop Block Diagram ................................................................ 73

Figure 53: DC Link Voltage Loop Root Locus........................................................................ 76

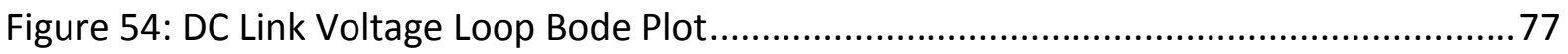

Figure 55: Standard PI and Anti-Windup PI Controller Responses.......................................78

Figure 56: Simulated Normalised Torque Reference for Turbine Emulator with Wind Shear 81

Figure 57: Simulated Normalised Torque for Tower Shadow ............................................82

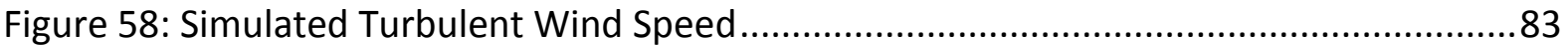


Figure 59: Simulated PMSG Current Step Response

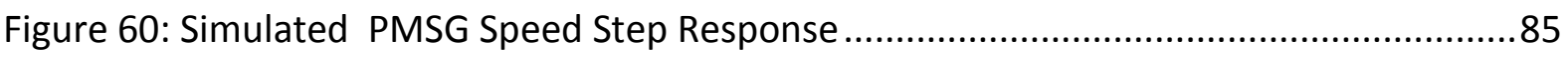

Figure 61: Simulated Grid Current Step Response ......................................................... 86

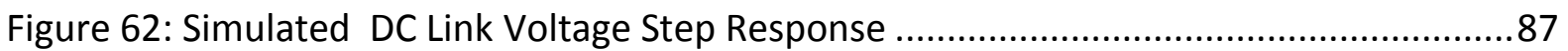

Figure 63: Simulated Effect of Wind Shear on Generator .................................................. 88

Figure 64: Simulated Effect of Tower Shadow on Generator ............................................89

Figure 65: Simulated Generator Parameters for All Turbine Effects .....................................90

Figure 66: Simulated Grid Current with Balanced Grid Voltages .......................................91

Figure 67: Simulated DQ Currents with Balanced Grid Voltages........................................92

Figure 68: Simulated Grid Currents with Type B Dip .........................................................93

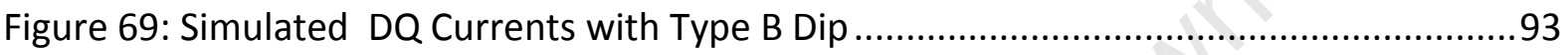

Figure 70: Simulated Dual Vector Control Grid Currents Balanced Grid Voltages .................94

Figure 71: Simulated Dual Vector Control DQ+ Currents with Balanced Grid Voltages..........95

Figure 72:Simulated Dual Vector Control DQ- Currents with Balanced Grid Voltages ...........95

Figure 73:Simulated Dual Vector Control Grid Currents with Type B Dip ............................96

Figure 74:Simulated Dual Vector Control DQ+ Currents with Type B Dip.............................97

Figure 75:Figure 74:Simulated Dual Vector Control DQ- Currents with Type B Dip ..............97

Figure 76: Simulated Wind Speed Turbulence with a Nominal Speed of $7 \mathrm{~m} / \mathrm{s}$....................98

Figure 77: Simulated Comparison Between Actual and Reference PMSG Speed ..................99

Figure 78: Simulated Cp During Turbulent Conditions ....................................................100

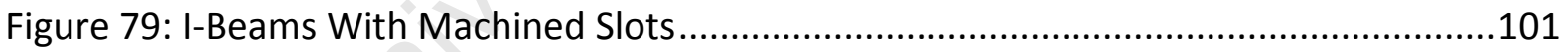

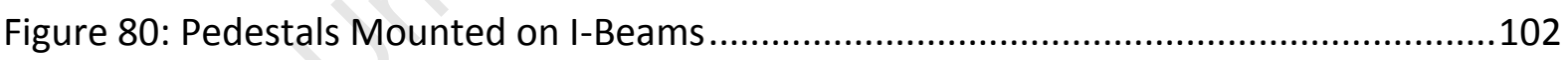

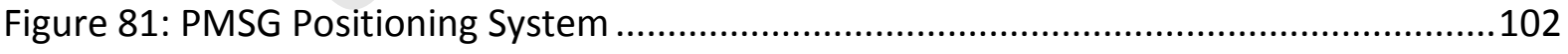

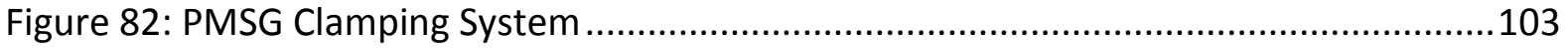

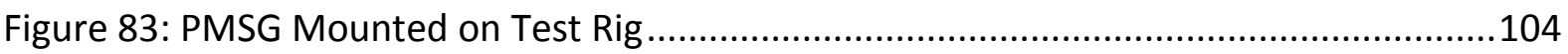

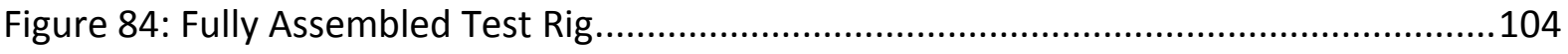

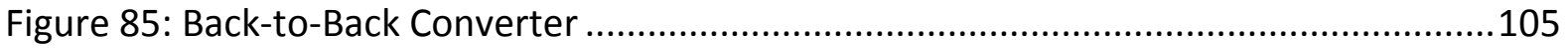

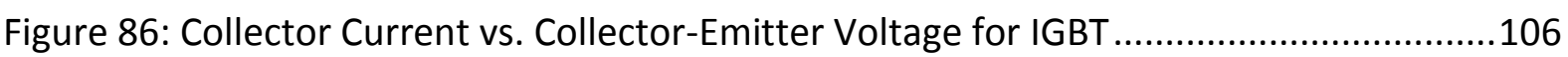

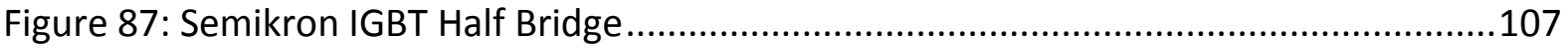

Figure 88: Semikron Driver Mounted on Converter .......................................................108

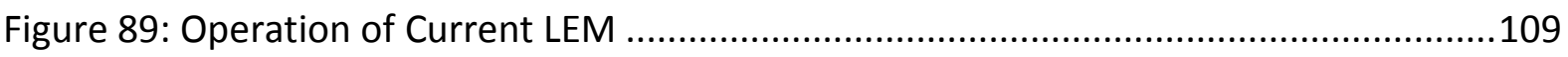

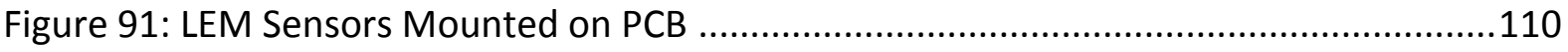




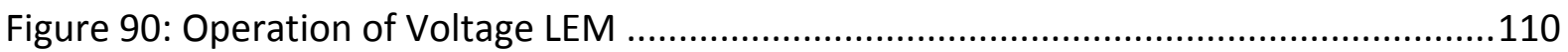

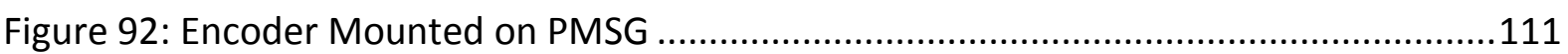

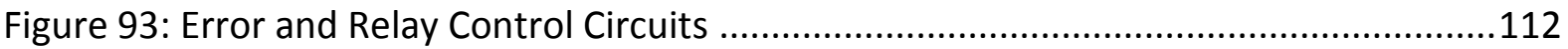

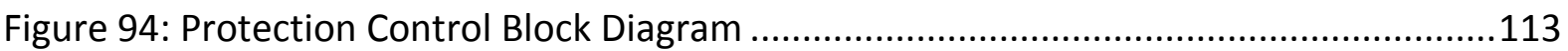

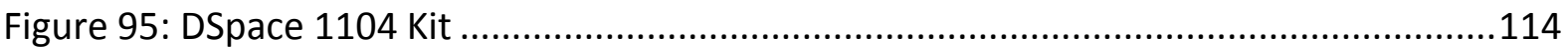

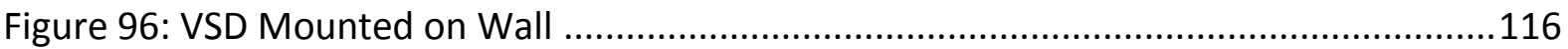

Figure 97: Dip Simulation Setup Block Diagram ..................................................................117

Figure 98: Dip Simulation Variable Transformers in Laboratory Setup ...............................117

Figure 99: Experimental Generator Current Step Response ….........................................120

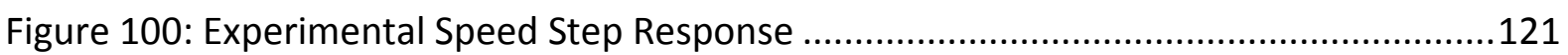

Figure 101: Experimental Grid Side Current Step Response ............................................122

Figure 102: Experimental DC Link Voltage Step Response ..............................................123

Figure 103: Experimental Effect of Wind Shear on Generator Parameters .........................124

Figure 104: Experimental Effect of Tower Shadow on Generator Parameters .....................125

Figure 105: Experimental Effect on Generator of All Turbine Effects .................................126

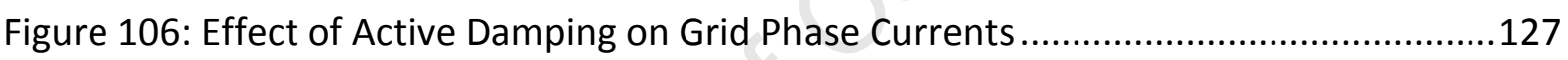

Figure 107: Grid DQ Currents with Active Damping Toggled On and Off...........................128

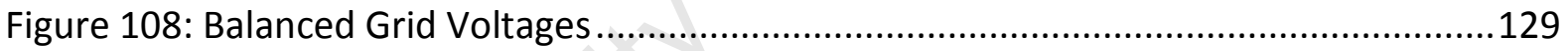

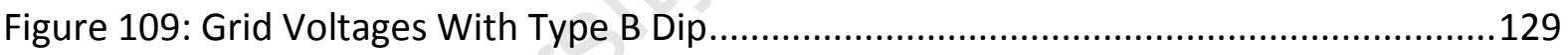

Figure 110: Experimental Grid Phase Currents Using Standard Vector Control with Balanced

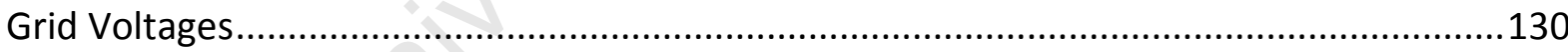

Figure 111: Experimental Grid DQ Currents Using Standard Vector Control with Balanced Grid Voltages.

Figure 112: Experimental Grid Phase Currents Using Standard Vector Control with Type B Dip

Figure 113: Experimental Grid DQ Currents Using Standard Vector Control with Type B Dip 132

Figure 114: Dual Vector Current Control Grid Phase Currents with Balanced Grid Voltages .134

Figure 115: Dual Vector Current Control Positive Sequence DQ Currents with Balanced Grid Voltages 134 
Figure 116: Dual Vector Current Control Negative Sequence DQ Currents with Balanced Grid

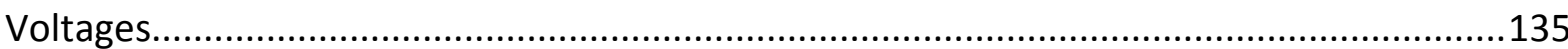

Figure 117: Dual Vector Current Control Grid Phase Currents with Type B Dip ..................136

Figure 118: Dual Vector Current Control Positive Sequence DQ Currents with Type B Dip.136

Figure 119: Dual Vector Current Control Negative Sequence DQ Currents with Type B Dip137

Figure 120: Experimental Wind Speed Under Turbulent Conditions .................................138

Figure 121: Comparison of Reference Speed with Actual Generator Speed .......................139

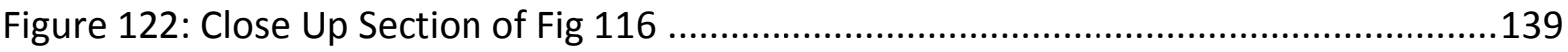

Figure 123: Experimental Power Coefficient Under Turbulent Conditions ...........................140

Figure 124: System Power at Various Stages During Turbulent Conditions..........................141

Figure 125: Experimental DC Link Voltage Under Turbulent Conditions ............................142

Figure 126: Rotor alignment with d-axis (a) and q-axis (b) [47] .......................................151

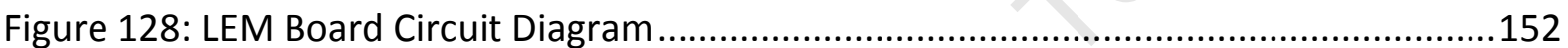

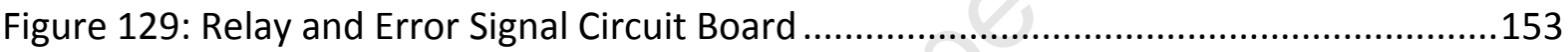




\section{List of Abbreviations}

\begin{tabular}{|c|c|c|}
\hline$A D C$ & - & Analogue to Digital Converter \\
\hline $\mathrm{CO}_{2}$ & - & Carbon Dioxide \\
\hline DAC & - & Digital to Analogue Converter \\
\hline DFIG & - & Doubly Fed Induction Generator \\
\hline EMF & - & Electromotive Force \\
\hline GWC & - & Growth Without Constraint \\
\hline $\mathrm{HCS}$ & - & Hill Climb Search \\
\hline IEA & - & International Energy Agency \\
\hline IG & - & Induction Generator \\
\hline IGBT & - & Insulated Gate Bipolar Transistor \\
\hline 10 & - & Input Output \\
\hline IPMSG & - & Interior Permanent Magnet Synchronous Generator \\
\hline IRP & - & Integrated Resource Plan \\
\hline LMA & - & Loss Minimisation Algorithm \\
\hline MPPT & - & Maximum Power Point Tracking \\
\hline MTA & - & Maximum Torque per Ampere \\
\hline O\&M & - & Operating and Maintenance \\
\hline $\mathrm{PI}$ & - & Proportional Integral \\
\hline PM & - & Permanent Magnet \\
\hline PMSG & - & Permanent Magnet Synchronous Generator \\
\hline PWM & - & Pulse Width Modulation \\
\hline REFIT & - & Renewable Energy Feed In Tariff \\
\hline RPM & - & Revolutions Per Minute \\
\hline SVPWM & - & Space Vector Pulse Width Modulation \\
\hline THD & - & Total Harmonic Distortion \\
\hline TSR & - & Tip Speed Ratio \\
\hline TTL & - & Transistor to Transistor Logic \\
\hline UPF & - & Unity Power Factor \\
\hline VC & - & Vector Control \\
\hline VSC & - & Voltage Source Converter \\
\hline WECS & - & Wind Energy Conversion System \\
\hline
\end{tabular}




\section{List of Symbols}

\begin{tabular}{|c|c|c|}
\hline$E_{\text {kinetic }}$ & - & Kinetic Energy \\
\hline $\mathrm{C}_{\mathrm{p}}$ & - & Power Coefficient \\
\hline $\mathrm{C}_{\text {pbetz }}$ & - & Theoretical Maximum Power Coefficient \\
\hline$B_{S}$ & - & Frictional Damping \\
\hline$C_{b}$ & - & Base Capacitance \\
\hline$C_{f}$ & - & Filter Capacitance \\
\hline$D_{p}$ & - & Pole Damping Factor \\
\hline$D_{z}$ & - & Zero Damping Factor \\
\hline$E_{n}$ & - & Line-to-line Voltage \\
\hline$F_{\text {res }}$ & - & Resonant Frequency \\
\hline$G_{M O}$ & - & Modulus Optimum Transfer Function \\
\hline$G_{r y}$ & - & Closed Loop Transfer Function \\
\hline$H_{F}$ & - & Shaping Filter \\
\hline$H_{L}$ & - & L-type Filter Transfer Function \\
\hline$H_{L C L}$ & - & LCL Filter Transfer Function \\
\hline$K_{i}$ & - & Integral Gain \\
\hline$K_{p}$ & - & Maximum Power Coefficient \\
\hline$K_{p}$ & - & Proportional Gain \\
\hline$L_{I}$ & - & Inverter Inductance \\
\hline$L_{T}$ & - & Total Filter Inductance \\
\hline$L_{b}$ & - & Base Inductance \\
\hline$L_{d}$ & - & d-axis Inductance \\
\hline$L_{g}$ & - & Grid Inductance \\
\hline$L_{\max }$ & - & Maximum Inductance \\
\hline$L_{q}$ & - & q-axis Inductance \\
\hline$P_{\text {actual }}$ & - & Actual Power \\
\hline$P_{b e t z}$ & - & Theoretical Maximum Attainable Power \\
\hline$P_{\text {grid }}$ & - & Real G rid Power \\
\hline$P_{\max }$ & - & Maximum Power \\
\hline$P_{m p p t}$ & - & Maximum Power Point \\
\hline$P_{n}$ & - & Rated Power \\
\hline$P_{\text {parallel }}$ & - & Power lost with Parallel Passive Damping \\
\hline
\end{tabular}




$$
\begin{aligned}
& P_{\text {series }} \quad \text { - Power lost with Series Passive Damping } \\
& P_{\text {total }} \quad \text { - Total Power } \\
& Q_{\text {grid }} \quad \text { - Reactive Grid Power } \\
& R_{T} \quad \text { - Total Parasitic Resistance } \\
& R_{d} \quad \text { - Damping Resister } \\
& T_{M O} \quad \text { - Modulus Optimum Time Constant } \\
& T_{d} \quad \text { - Lead-Lag Time Constant } \\
& T_{e} \quad-\quad \text { Electrical Torque } \\
& T_{g} \quad \text { - Generator Torque } \\
& T_{i} \quad-\quad \text { Integral Time Constant } \\
& T_{\text {load }} \quad-\quad \text { Load Torque } \\
& T_{\text {opt }} \quad \text { - Optimal Torque } \\
& V_{h} \quad-\quad \text { Wind Speed } \\
& V_{\text {CEstat }} \quad \text { - Collector-Emitter Monitoring Voltage } \\
& V_{\text {dconv }} \quad \text { - d-axis Converter Voltage } \\
& V_{\text {dgrid }} \quad \text { - } \mathrm{d} \text {-axis Grid Voltage } \\
& V_{\text {qconv }} \quad \text { - q-axis Converter Voltage } \\
& V_{\text {qgrid }} \quad \text { - } \text { q-axis Grid Voltage } \\
& W_{c u} \quad \text { - Copper Losses } \\
& Z_{b} \quad \text { - Base Impedance } \\
& a_{i} \quad-\quad \text { Coefficient of } \mathrm{C}_{\mathrm{p}} \\
& f_{\max } \quad-\quad \text { Maximum Frequency } \\
& i_{a} \quad \text { - Phase A Current Phasor } \\
& i_{b} \quad \text { - Phase B Current Phasor } \\
& i_{c} \quad \text { - Phase C Current Phasor } \\
& i_{d} \quad \text { - Direct-axis Current } \\
& i_{d-} \quad-\quad \mathrm{d} \text {-axis Negative Sequence Current } \\
& i_{d+} \quad-\quad \mathrm{d} \text {-axis Positive Sequence Current } \\
& i_{g}(h) \quad \text { - } \quad \text { Grid Harmonic Current } \\
& i_{q} \quad \text { - Quadrature-axis Current } \\
& i_{q-} \quad \text { - } \quad \text { q-axis Negative Sequence Current } \\
& i_{q+} \quad \text { - } \quad \text {-axis Positive Sequence Current } \\
& i_{s} \quad \text { - Current Space-Phasor } \\
& i_{\alpha} \quad \text { - Alpha-axis Current }
\end{aligned}
$$




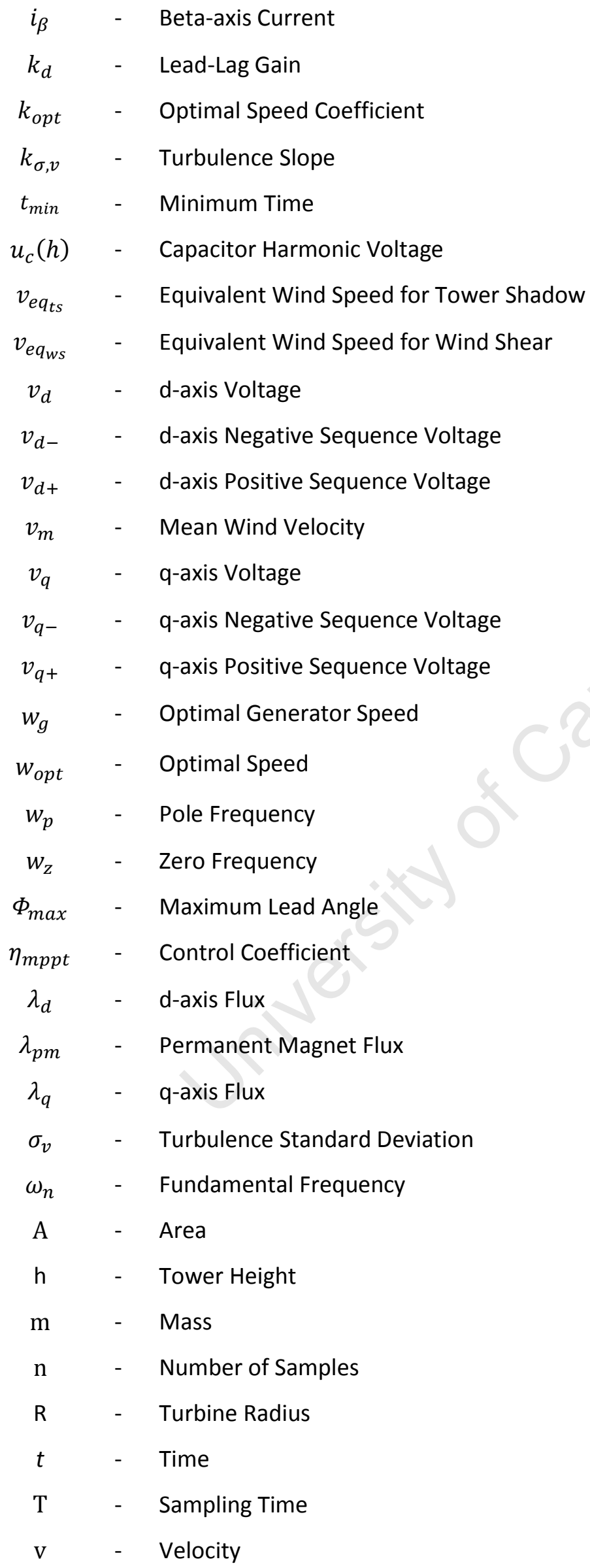




$\begin{array}{cll}\rho & - & \text { Air Density } \\ E & - & \text { Energy } \\ G & - & \text { Notch Filter Transfer Function } \\ J & - & \text { Inertia } \\ L & - & \text { Length of Turbulence } \\ L(s) & - & \text { Lead-Lag Transfer Function } \\ P & - & \text { Power } \\ R & - & \text { Resistance } \\ i(h) & - & \text { Inverter Harmonic Current } \\ p & - & \text { Number of Pole Pairs } \\ r & - & \text { Blade Radius } \\ v & - & \text { Wind Velocity } \\ w & - & \text { Turbine speed } \\ \alpha & - & \text { Emperical Wind Shear Component } \\ \zeta & - & \text { Damping Factor } \\ \theta & - & \text { Azimuthal Angle } \\ \lambda & - & \text { Tip Speed Ratio } \\ & & \end{array}$




\section{CHAPTER 1}

\section{INTRODUCTION}

\subsection{Background}

It is widely accepted that green-house gas (GHG) emissions from the combustion of fossil fuels for electricity production has contributed to global warming and climate change. Consequently, a global effort to meet our electricity needs from clean and renewable energy sources has emerged. At the same time, recent advances in technology has resulted in renewable energy becoming increasingly reliable and economically viable. The World Wildlife Fund (WWF) predicts that renewable energy could supply $100 \%$ of the world's electricity needs by 2050 [1]. South Africa's electricity demand is reaching the limit of its supply capacity and the generation infrastructure is aging. Thus, if no additional infrastructure is installed, there will be rolling blackouts predicted from 2011 until 2016, which will have a serious impact on the economy. These circumstances have led to significant research and development into augmenting South Africa's energy mix with renewable energy generation over the next 2 decades [2].

\subsubsection{Electricity Generation in South Africa}

South Africa has a population of 50 million occupying an area of $1.2 \times 10^{6} \mathrm{~km}^{2}$. It is a country which is rich in minerals, including platinum, gold, diamonds, coal, uranium, etc. The electricity generation, transmission and distribution infrastructure is owned and operated by the state utility, Eskom, which currently has an installed capacity of $43 \mathrm{GW}$. Over the past few years the electricity reserve margin has dropped to approximately $8 \%$ which is well below the accepted norm of $15 \%$ [3]. South Africa is enormously reliant on coal for its electricity production as illustrated in Table 1 below. As a result of this, South Africa is one of the largest contributors to world $\mathrm{CO}_{2}$ emissions from the combustion of fossil fuels. According to a study conducted in 2006, it ranking $15^{\text {th }}$ amongst the global $\mathrm{CO}_{2}$ emitters as illustrated in Figure 1 below [4]. 
Table 1: Current Energy Mix of South Africa [5]

\begin{tabular}{|l|l|l|}
\hline \multicolumn{2}{|l|}{ Generation Type } & MW $\%$ \\
\hline Coal & 37755 & 86 \\
\hline Nuclear & 1930 & 4.4 \\
\hline Gas & 2426 & 5.5 \\
\hline Pumped Storage & 1400 & 3.4 \\
\hline Wind & 3 & 0.007 \\
\hline
\end{tabular}

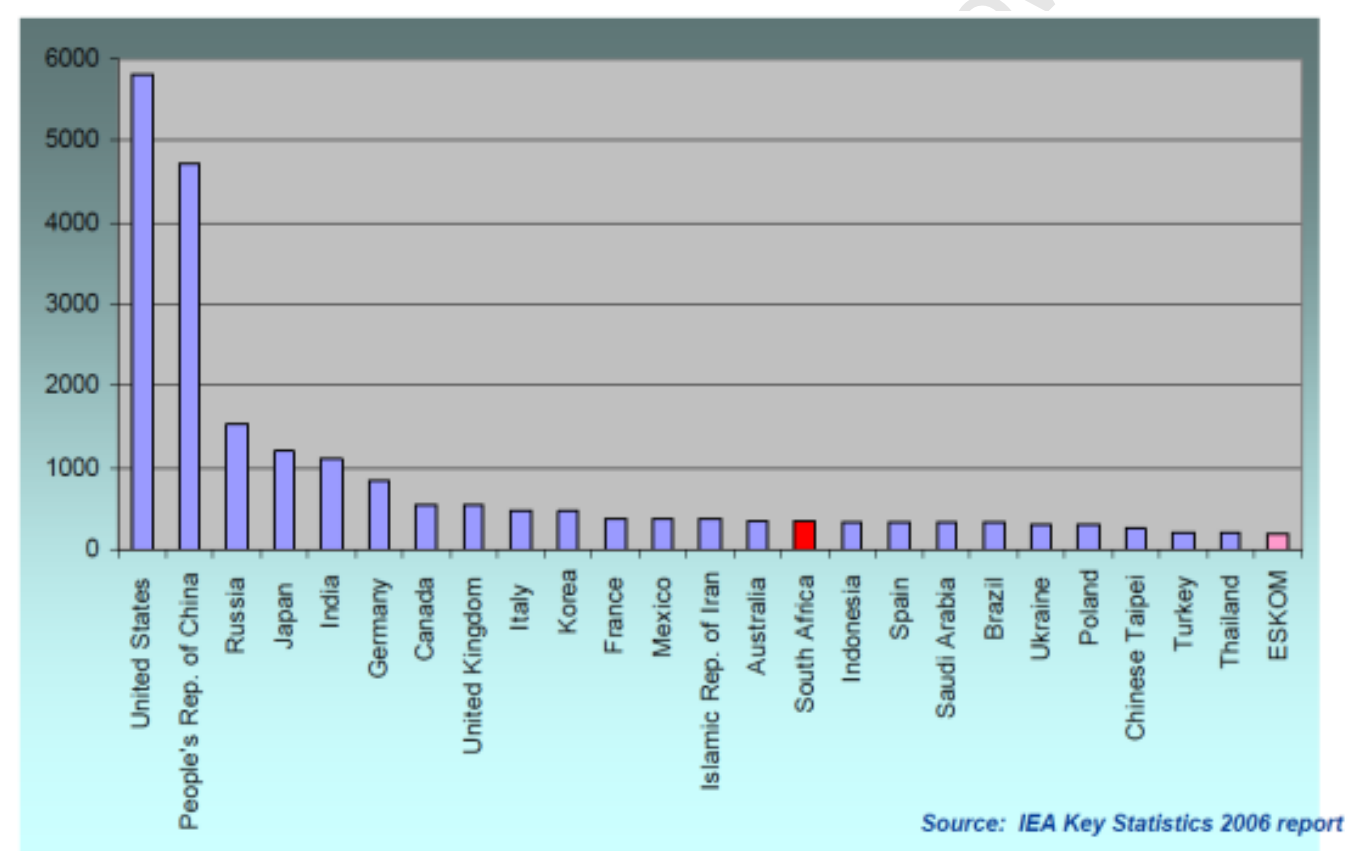

Figure 1: Million ton of $\mathrm{CO} 2$ emission from the burning of fossil fuels in 2006 [4]

\subsubsection{Future Energy Mix}

In an attempt to reduce South Africa's carbon emissions, the IRP (Integrated Resource Plan) was developed to plan the country's energy mix over the next 20 years. This included a goal to expand the total installed capacity to more than $80 \mathrm{GW}$ by 2030 . However, a compromise was to be made between least-investment cost, climate change mitigation, diversity of 
supply, localization and regional development. Many scenarios were analyzed during the development process of the IRP, which included two extreme cases, namely a low cost scenario and a low carbon scenario. A compromise between the two is made with a balanced scenario. Table 2 illustrates the proposed energy mix for South Africa by 2030, according to the latest IRP 2030 [6].

Table 2: IRP 2030 Energy Mix with Balanced Scenario

\begin{tabular}{|l|l|}
\hline \multicolumn{2}{|c|}{$\%$} \\
\hline Baseload Coal & 48 \\
\hline Renewables(Wind, Solar etc) & 16 \\
\hline Baseload Nuclear & 14 \\
\hline Peaking OCGT & 9 \\
\hline Peaking Pump Storage & 6 \\
\hline Mid-Merit Gas Power & 5 \\
\hline Baseload import Hydro & 2 \\
\hline
\end{tabular}

Wind energy will play a major part in the renewable segment of the energy mix. This is largely due to the maturity of the technology and the fact that South Africa has a large coastal region where higher wind speeds are usually found. A wind map of South Africa, as shown in Figure 2, which indicates that the West Coast has favourable wind speeds with an average of $6 \mathrm{~m} / \mathrm{s}$, at $10 \mathrm{~m}$ above the ground. In addition to this, it is estimated that the wind potential of South Africa exceeds 50GW [7]. 


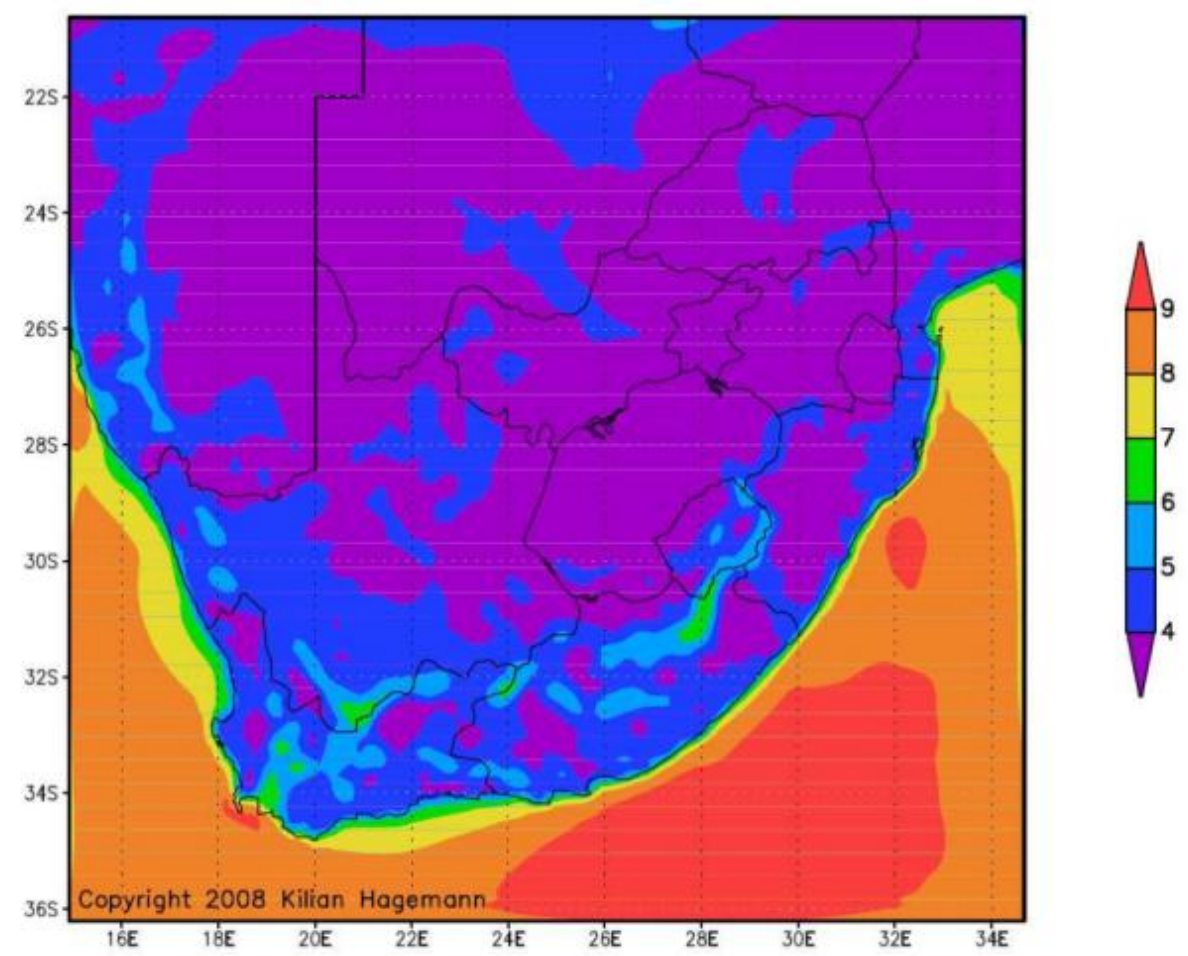

Figure 2: Average annual wind speeds at $10 \mathrm{~m}$ above ground in $\mathrm{ms}-1$ [7]

South Africa has sufficient wind capacity however there are other important factors to consider before large scale wind energy can be incorporated in its energy mix. These factors include: the cost of wind energy, short term effects on grid integration as well as the effect it will have on the greater economy. Brief discussions of each of the factors will be presented in the following sections.

\subsubsection{Cost Trends}

The cost of energy from wind power plants is affected by five primary factors which include: annual energy production, investment costs, operating and maintenance (O\&M) costs, financing costs and the economic life of the plant.

Wind resources vary from location to location as well as over a period of time. These factors determine how productive a wind energy plant will be over the duration of a year. Thus, precise locations need to be selected for wind farms and even single wind turbines to maximise production. Other factors that affect the annual energy production are turbine components such as rotor diameter and tower height. The trend is to increase the size of these components and therefore increase the annual energy production of every turbine. As new technologies are developed and the size of turbines increases, the price of wind power generation is decreasing. Smaller turbines $(<500 \mathrm{~kW})$ that are commercially available cost 
approximately three times the price of large utility turbines $(>1.5 \mathrm{MW})$ [8]. The increase in turbine size does however lead to the problem of large upwind turbines effecting downwind turbine production and has to be taken into consideration [9].

The initial investment required to setup a wind power plant requires a large amount of capital and accounts for between $75-80 \%$ of the total cost of the wind farm over its life span. The remaining costs are made up of O\&M. The investment cost includes the cost of the turbines, grid connection, civil works, and other costs such as licensing and environmental assessments [9]. Table 3 provides a breakdown of the investment cost for a wind power plant with turbine costs comprising more than $70 \%$ of total investment costs for onshore wind power plants. The remaining investment costs are determined by the plant location. Design improvements and an increase in turbine scaling can decrease the initial investment costs.

Table 3: Initial Investment Cost Split [9]

\begin{tabular}{|l|l|}
\hline \multicolumn{2}{|l|}{ Component } \\
\hline Turbine & $71-76$ \\
\hline Grid Connection & $10-12$ \\
\hline Civil Works & $7-9$ \\
\hline Other Investment Costs & $5-8$ \\
\hline
\end{tabular}

The operation and management (O\&M) costs are made up of fixed costs and variable costs. Fixed costs consist of land leases, insurance, taxes, management, and forecasting services. The variable costs are linked to maintenance and spare parts. The O\&M costs increase in the following years because of manufacturer warranties that expire as the turbine ages [9].

Financing arrangements, including the cost of debt and equity can also influence the cost of wind energy. Predictability of the policies supporting wind energy such as REFIT can have a large impact on financing costs as well as investment interest [9]. In addition, the expected operating life of the wind power plant also has influence on costs. 


\subsubsection{Job Creation}

South Africa's unemployment rate is currently $25 \%$ and the government is developing a "New Growth Path" which aims to create 5 million jobs by 2020. Any new developments and their ability to create jobs play a role in whether or not they are pursued. With this in mind, studies have been conducted to predict what impact renewable energy would have in terms of job creation as well as the effect it would have on current job sectors such as the coal industry. Initial indications based on job creation in other parts of the world show positive signs. It is estimated that the wind industry currently employs 400,000 workers worldwide as well as it having positive effects in rural areas [10].

Greenpeace carried out a study for South Africa in [11]. In the study they introduced and compared three scenarios.

- The Energy [R]evolution which predicts a low carbon footprint future. Renewable energy will supply $36 \%$ of South Africa's electricity by 2030 .

- A reference case from the International Energy Agency (IEA) energy projection for Africa. In this case South African emissions almost double by 2050.

- The 'Growth Without Constraints' (GWC) scenario. This scenario would increase South Africa's emissions four-fold by 2050.

Although the Energy [R]evolution would cause job losses in the nuclear and coal industries, it was predicted that it could outperform the other scenarios in terms of job creation as illustrated in Figure 3. However, it is possible that the coal trade industry will suffer losses regardless because of carbon taxes as well as importing countries needing to meet their emission goals. The study suggests that employing renewable energy could contribute to the government's goal of job creation while at the same time reducing the county's carbon emissions. 


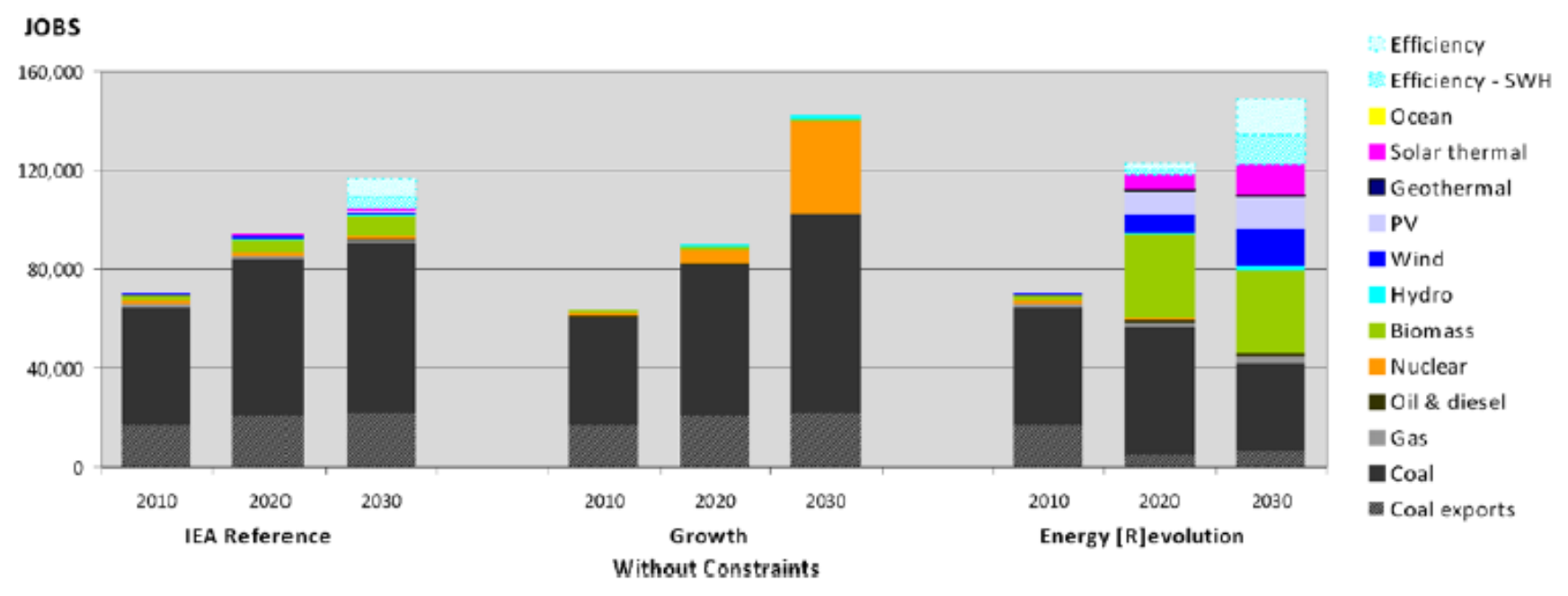

Figure 3: Jobs by Technology and Type 2010, 2020 and 2030 [11]

\subsubsection{Near Term Grid Integration Issues}

Wind energy is variable and dependant on the weather, therefore the grid that it is integrated with needs to be flexible in order to deal with the varying energy supply. The deeper wind energy penetrates the more important complex technologies such as smart grids and wind forecasting becomes [8]. In this regard, countries where considerable wind energy rollout has occurred have found that low to medium levels of wind penetration up to $20 \%$ poses no overwhelming economic and technical barriers in terms of integration [9].

Accurate forecasting of wind can improve the effectiveness of the system. In [12], a smoothing effect of the energy variability is observed as the number of plants increases. They do however have to be decentralised over a wide area. The smoothing effect allows for an increase in the accuracy of the forecast.

Another issue that arises is the fact that areas that are ideal for wind energy plants are located in remote areas far from the electricity demand. Choices have to be made on whether it is more economically viable to build additional transmission infrastructure or to use lower-quality wind resources that are closer to the demand [9].

When considering all of the above, and the fact that South Africa will be expanding its wind turbine fleet in the near future, a system that can emulate the behaviour of wind turbines in a laboratory environment can be beneficial for solving complex issues that are experienced in the industry. This is the motivation behind the presented thesis. 


\subsection{Literature Review}

The project is a continuation of previous work done in the research group by Stefan Sager. The limitations of his work include hardware restrictions, controller design and testing of the system using wind data. This section provides a brief review of the literature that is applied to this project in order to keep up with the latest trends and techniques. The concepts are discussed further in chapters 2, 3 and 4.

There are many types of control that are implemented in literature for a permanent magnet synchronous generator WECS and the type of control is dependent on the converter topology. In [15], the different types of WECS topologies are evaluated and compared. A common technique that offers a wide variety of control options is the back-to-back full bridge converter. The converters are usually controlled using vector control.

It is also important to operate generator efficiently in order to minimise system losses. There are various control techniques that can be embedded within the vector control in order to achieve efficient operation and the Maximum Torque Per Ampere method is chosen for this project. Other techniques such as the Unity Power Factor control is introduced in [45] while the Loss Minimisation Algorithm is discussed in [46].

Voltage Source Converters (VSC) can produce waveforms with low total harmonic distortion but introduce high frequency harmonic distortion at their switching frequency. These harmonics occur at frequencies that are well above the fundamental grid frequency and can be removed by means of a grid filter. Common filter topologies are $L$ and LCL filters, the latter being used for higher power systems because of its reduced size[27][28][29]. LCL filters introduce resonance which can lead to instability of the system. Damping methods are employed that can either be active or passive in nature. Passive damping uses resistors that can be situated in various positions throughout the filter. This technique is generally avoided in high power systems because a sizeable amount of power is dissipated through the resistors. Passive damping makes use of no extra filter components but is rather implemented within the control algorithms. Some techniques require extra sensors [31][32] while others are sensorless [33]. In [30], a review and comparison of various damping techniques is conducted. 
The most important part of any control algorithm is being able to tune the controller parameters effectively. This allows the algorithm to perform optimally. The controller used in literature are simple PI controllers and can be tuned using methods which are introduced elaborated on in [39][41][42]. The inner control loops are controlled using the modulus optimum and the outer voltage/speed loops are controlled using the symmetrical optimum method.

In order to extract the maximum available power from the wind, Maximum Power Point Tracking (MPPT) is employed. There are various techniques that can be used to achieve this that can be achieved into two different groups. The first uses the parameters of the turbine to determine the optimal rotational speed for every wind velocity [18]. Systems that use this technique have very accurate control but generally need to make use of a wind sensor. The second technique is a search based algorithm that makes small changes to the turbine speed and monitors the output power trying to maximise it [19][20]. This technique does not require any knowledge of the wind turbine or the wind speed but can introduce oscillations into the system.

It is important to understand wind and the effects that it has on a wind turbine. There are factors such as wind shear, tower shadow, tower height and turbine inertia that have an impact on the power quality that is extracted from the turbine. In [22] a wind model is developed that includes the pulsating characteristics of wind shear and tower shadow. It allows for the effects to be implemented within a turbine emulator. Turbulence is introduced in [21], which is used to test the overall effectiveness of the system control.

Wind turbines are required to handle certain power quality issues associated with the grid because they are being increasingly relied upon as a base load energy source. In order to analyse the power quality issues in a tangible manner, the waveforms are converted into their positive and negative sequence values. The work of [36] introduces two methods that are used for sequence extraction. The positive sequence component indicates the portion of the three phase waveform that is perfectly balanced. Any unbalance is indicated by the presence of a non-zero negative sequence component. An alternative control technique is made available by utilising positive and negative sequences. Dual vector control is implemented in [38] to deal with the power quality issues. It uses four current loops in place 
of the two that standard vector control uses. This introduces extra complexity to the control algorithm but also introduces greater control of the output waveforms from the converter.

\subsection{Research Questions}

The research presented in this thesis focuses on the full understanding and implementation, both in simulations and experimentally of a Permanent Magnet Synchronous Generator (PMSG) Wind Turbine System.

The research presented in this thesis explores the implementation of a Permanent Magnet Synchronous Generator (PMSG) Wind Turbine System, focusing on optimising the control that is utilised as well as the system performance under unbalanced grid conditions.

Several research questions have been put forward:

- Which system topologies exist for WECS?

- How can a wind turbine be emulated in the laboratory?

- How do you control a PMSG?

- How can a WECS be connected to the grid?

- What is required from a grid connected WECS?

\subsection{Objectives}

The objectives of the work carried out are to:

- Implement a wind turbine emulator in the laboratory for the research project.

- Develop and implement the control of the PMSG.

- Investigate and identify how a power electronic converter connects to the grid.

- Implement the WECS experimentally in the laboratory by combining the individual components.

- Identify what is required from a WECS in terms of grid codes.

- Validate the operation of the WECS experimental setup. 


\subsection{Scope and Limitations}

A 6kW PM wind turbine system was available for this project. No operating range limits were placed on the system. Operation under grid faults was limited to voltage dips less than $15 \%$.

\subsection{Structure}

An introduction to WEC systems in presented in chapter 2 including the modelling of a turbine and the requirements for connecting to the grid. In chapter 3 , the control of the generator and grid side converters is discussed. Filter and controller design is introduced and implemented in chapter 4 . The components of the system are simulated in chapter 5. Chapter 6 discusses the implementation of the system in the laboratory. Analysis of the results and carried out in chapter 7 . Conclusions and recommendations are presented in chapter 8 . 


\section{CHAPTER 2}

\section{WIND ENERGY CONVERSION SYSTEM THEORY DEVELOPMENT}

\subsection{WECS Topologies}

There are various WECS topologies with different generator configurations. Among them, induction generators are popular for high power systems in the MW range and therefore are used in the larger wind turbines. They exhibit numerous advantages including low cost compared to PM generators and very good reliability with low maintenance requirements. The main limitation is that they require reactive power and therefore need a bidirectional converter. Some induction generators are controlled by accessing the rotor windings and are called doubly fed induction machine (DFIG). PM generators do not require reactive power, and therefore have a greater variety of converter topologies available to them. They are popular for smaller wind energy conversion systems because they do not require external excitation current to operate. They are also able to operate without a gearbox and can be directly coupled to the turbine blades. PM generators have been considered for larger WECS but the price of the rare earth magnets render them economically unviable.

Figure 4 shows the schematic of an IG connected to the grid with a full bridge back-to-back converter.

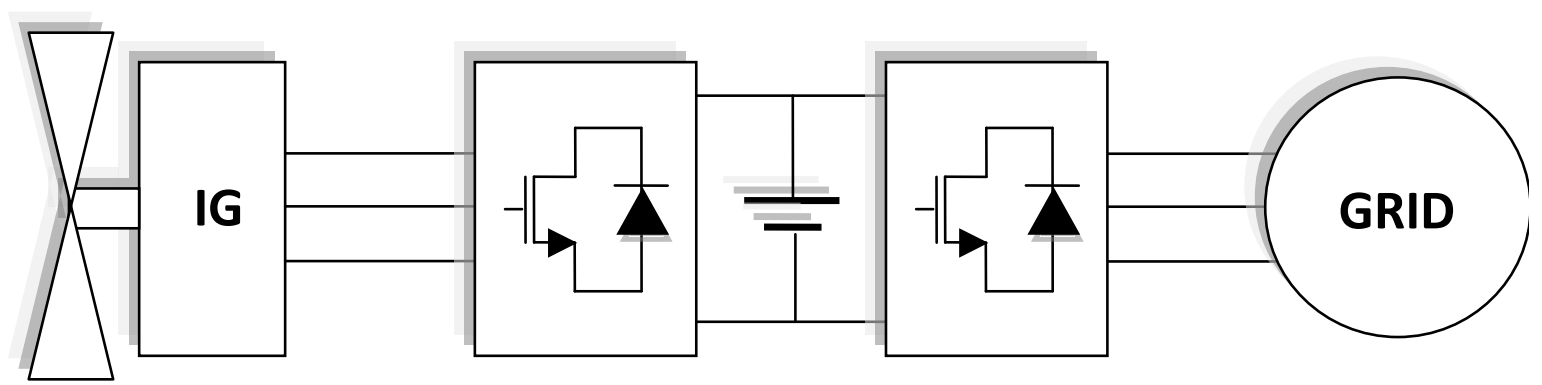

Figure 4: Induction Generator with a Back-to-Back Converter Topology 
This type of converter has the ability for bidirectional power flow and is able to supply the reactive power required by these generators. The torque and the speed are controlled by the generator side converter currents allowing MPPT to be implemented. The grid side of the converter maintains a constant DC link voltage by transferring excess power to the grid. In Figure 5, a DFIG schematic is shown and most of the power flows through the stator which is connected directly to the grid. Consequently, a converter with about one third of the rated generator power can be used.

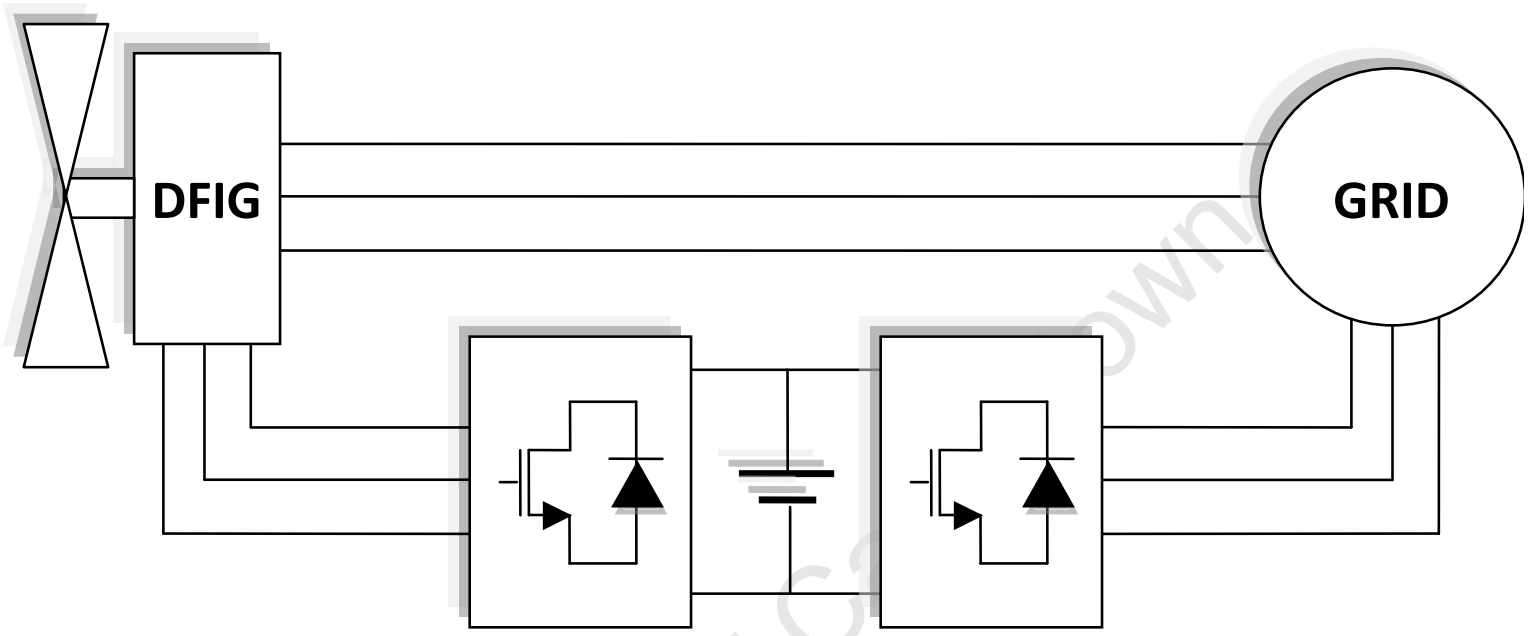

Figure 5: Doubly-Fed Induction Generator with a Back-to-Back Converter Topology

A major advantage of using a PMSG is that it does not require any reactive power and a bidirectional converter is not required. A diode rectifier can be used on the machine terminals greatly simplifying the control as well as reducing the cost of the system as fewer switches are required. This type of a system is implemented in [13] and illustrated in Figure 6.

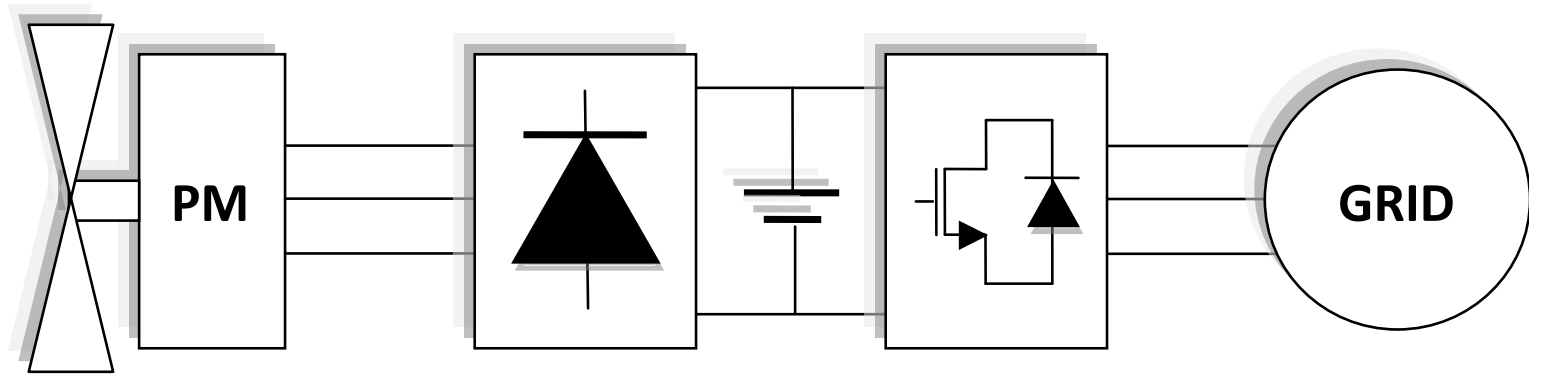

Figure 6: Permanent Magnet Synchronous Machine with a Diode Rectifier-VSI Converter Topology 
MPPT is implemented with a power mapping technique that relies on the relationship between the DC link voltage and the output power of the generator.

A modification to the previous topology is made in [14] where a DC-DC converter is added after the diode rectifier as is illustrated in Figure 7. The additional converter is used to control the DC link currents and hence the generator currents, which allows for MPPT to be implemented in the same way as the IG and DFIG topologies.

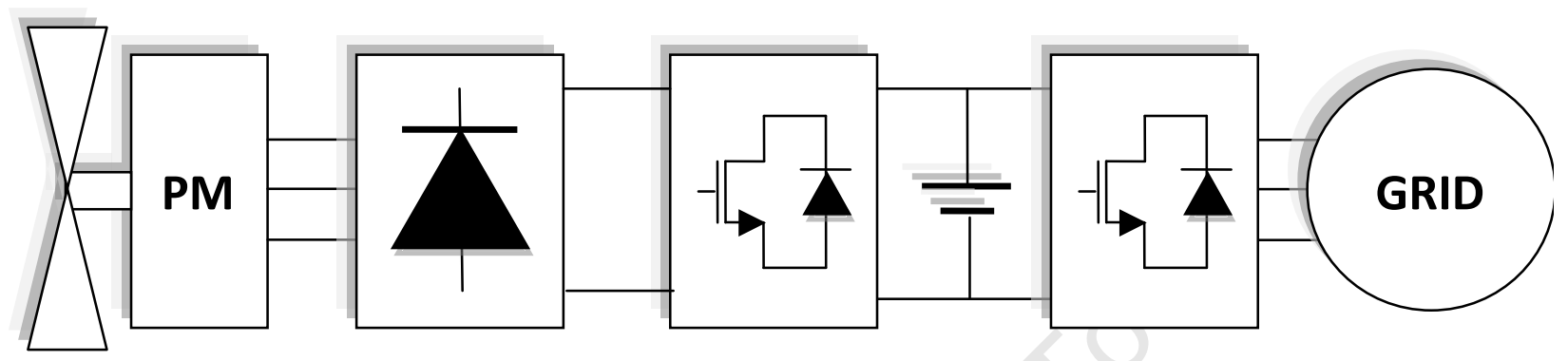

Figure 7: Permanent Magnet Synchronous Machine with a Diode Rectifier-Chopper-VSI Converter Topology

Figure 8 illustrates the full bridge back-to-back converter topology connected to the PMSG. It performs the same functions in terms of current control, voltage regulation and MPPT as when it is connected to the IG and DFIG.

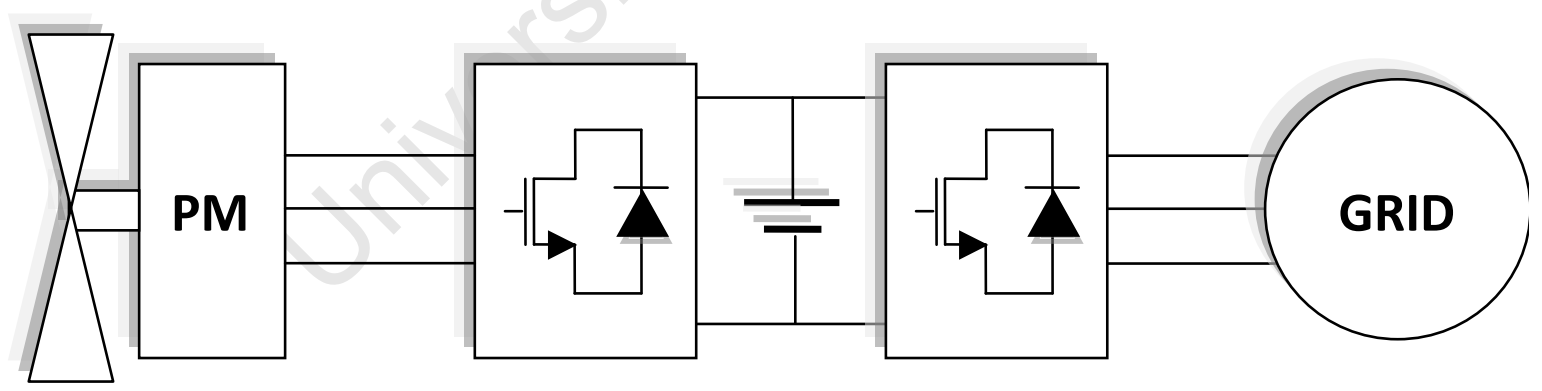

Figure 8: Permanent Magnet Synchronous Machine with a Back-to-Back Converter Topology

Every topology has its advantages and disadvantages, whether it is cost or control flexibility and the configuration that is chosen will have to take all these factors into consideration. $\mathrm{A}$ comprehensive summary and comparison of WECS topologies is conducted in [15]. 


\subsection{Turbine Basics}

This section aims to introduce the basic concepts of wind turbine operation. The ideas of maximum attainable energy, tip speed ratio as well as the different MPPT techniques are investigated.

\subsubsection{Power in the Wind}

The total power of a moving air mass can derived from the basic formula of kinetic energy which is given by equation 2.1. The derivation of power from energy is stated in equation 2.2 , where $t$ is time. By combining equations 2.1 and 2.2 we can determine the total power which is given by equation 2.3 .

$$
\begin{gathered}
E_{\text {kinetic }}=\frac{1}{2} \mathrm{~m} \cdot \mathrm{v}^{2} \\
P=\frac{E}{t} \\
P_{\text {total }}=\frac{1}{2} \rho \cdot \mathrm{A} \cdot \mathrm{v}^{3}
\end{gathered}
$$

Where $\rho$ is the air density, $A$ is the area swept by the blades, $v$ is the wind velocity, mass $m=\rho$. A. $\mathrm{d}, \mathrm{d}=$ distance and velocity $v=\frac{d}{t}$.

The kinetic energy from the moving air mass is transferred into mechanical-rotational energy in the wind turbine. This transfer occurs when the turbine slows the moving air mass although not all the energy can be extracted from the wind otherwise the air mass would have to stop moving completely. In 1926, Betz discovered that a theoretical maximum of $59 \%$ of the air mass energy could be captured by a wind turbine and is expressed in equation 2.4 [16].

$$
P_{\text {betz }}=\frac{1}{2} \rho \cdot \mathrm{A} \cdot \mathrm{v}^{3} \cdot \mathrm{C}_{\mathrm{pbetz}}
$$


Where $C_{\text {pbetz }}=0.59$. The actual value captured by the wind turbine is expressed by equation 2.5 where $C_{p}$ is the power coefficient.

$$
P_{\text {actual }}=\frac{1}{2} \rho \cdot \mathrm{A} \cdot \mathrm{v}^{3} \cdot \mathrm{C}_{\mathrm{p}}
$$

\subsubsection{Power Coefficient $C_{p}$ and Tip Speed Ratio $\lambda$}

The Power Coefficient is the ratio of the actual power and the theoretical maximum power and is expressed in terms of tip speed ratio $\lambda$ and pitch angle $\beta$.

$$
\mathrm{C}_{\mathrm{p}}=\frac{P_{\text {actual }}}{P_{\text {betz }}}
$$

The pitch angle refers to the angle that the turbine blades are aligned to and the blades can be pitched in or out of the wind depending on the available wind energy. Some turbines use a fixed pitch and the power coefficient becomes a function of $\lambda$ only. The equation of $C_{p}$ under fixed pitch conditions can be represented by equation 2.7 where $a^{i}$ is the coefficient of $C_{p}$.

$$
C_{p}=\sum_{i=0}^{n} a_{i} \lambda^{i}
$$

The Tip Speed Ratio $\lambda$ can be represented by equation 2.8 and refers to the ratio between the wind speed and the turbine blade speed.

$$
\lambda=\frac{R \cdot w}{v}
$$

Where $\mathrm{R}$ is the turbine radius and $w$ is the speed at which the turbine is rotating. The relationship of $C_{p}$ and $\lambda$ that was used for this project is illustrated in Figure 9. 


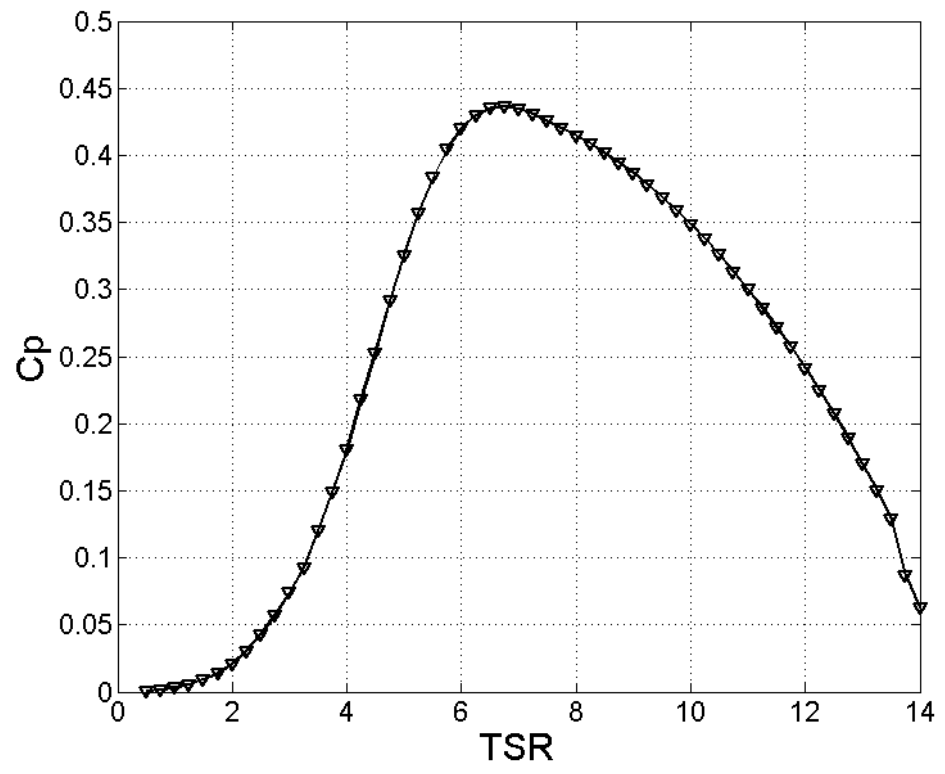

Figure 9: Power Coefficient vs. Tip Speed Ratio

\subsubsection{Maximum Power Point Tracking (MPPT)}

The objective of MPPT is to develop as much mechanical power from the available wind as possible. Analysing equation 2.5 , the only controllable parameter is the power coefficient $C_{p}$. Furthermore, Figure 10 indicates that for a given wind speed, there will be a tip speed ratio that will produce the maximum value for $C_{p}$.

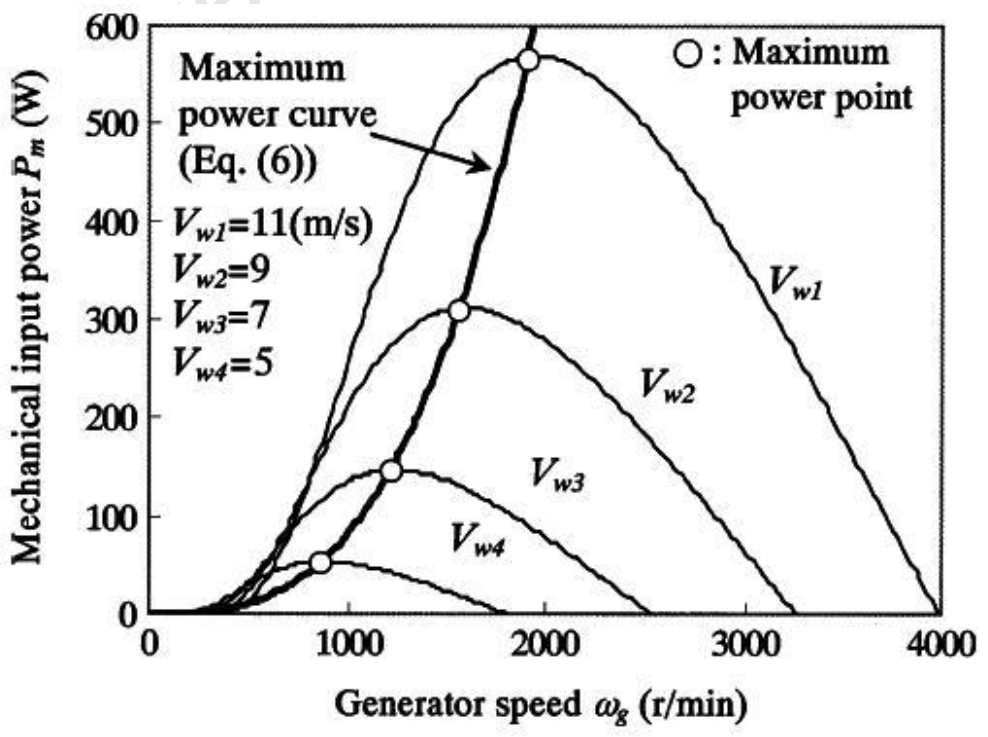

Figure 10: Power-Speed Curve 
Therefore, the tip speed ratio, and consequently the speed of the turbine, needs to be controlled in order to maximise the harnessed mechanical power. There are three common methods that are used for MPPT in wind turbines. The first uses the Power-Speed curve where the speed of the generator is controlled to generate the maximum power for every wind speed [17] [18]. The curve is illustrated in Figure 10 and equation 2.9 is used to obtain the maximum power where $K_{p}$ is the maximum power coefficient. The generator speed is controlled to track the maximum power curve.

$$
P_{\max }=K_{p} \mathrm{w}^{3}
$$

The second method utilises the Torque-Speed curve which is illustrated in Figure 11 [18].

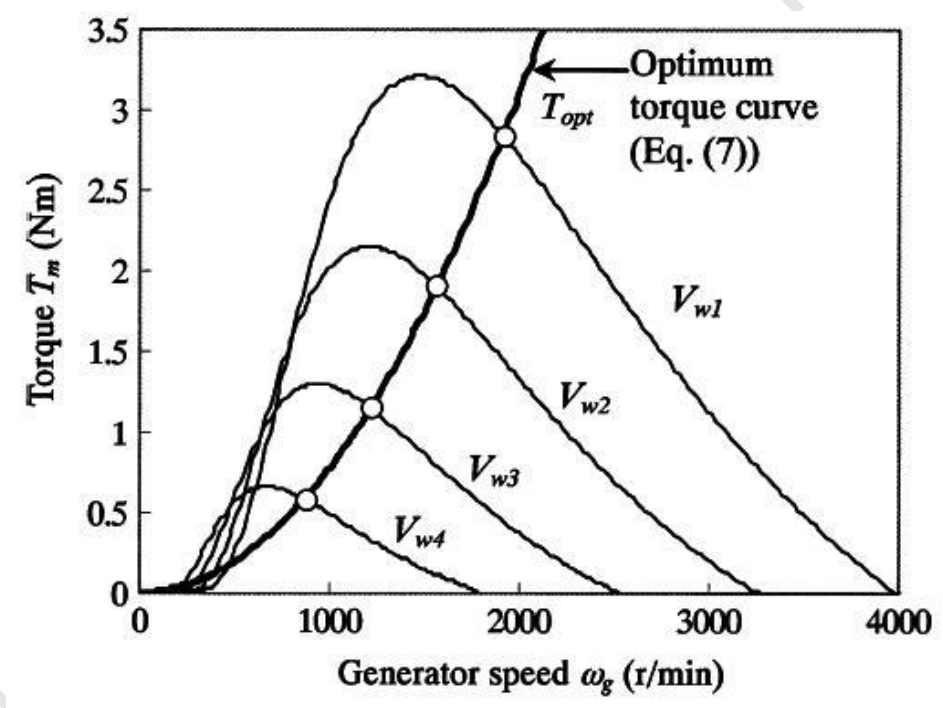

Figure 11: Torque-Speed Curve

The maximum power is achieved by controlling the torque of the machine. When the optimal torque for a specified wind velocity is produced, the system will settle at the optimal speed, producing the maximum achievable power. If the mechanical torque produced by the turbine blades is greater than the generator torque, the generator speed will increase to draw nearer to the optimal speed. If the generator torque is greater than the mechanical torque, the generator speed will decrease to draw nearer to the optimal speed. 
Equation 2.10 shows the relationship between the optimal torque $T_{o p t}$ and the optimal speed $w_{\text {opt }}$ where $k_{\text {opt }}$ is the optimal speed coefficient. The generator torque command is calculated using the control law in equation 2.11 .

$$
\begin{aligned}
& T_{o p t}=k_{o p t} w_{o p t}^{2} \\
& T_{g}=k_{o p t} w_{g}^{2}
\end{aligned}
$$

A third approach for MPPT is the Hill Climb Search (HCS) which attempts to climb the PowerSpeed curve. It changes the system speed in an attempt to increase the power. If the change in speed results in a positive increase in power, then it is climbing the hill in the correct direction. This method is the most generalised method of the MPPT's as it does not require any of the turbine or generator parameters.

The generalised nature of this method can cause some problems. The first is the step-size and efficiency trade off [19]. The larger the perturbations, the quicker the algorithm will converge however it will decrease the control efficiency, $\eta_{m p p t}$ as well as cause overshoot which is illustrated in Figure 12.

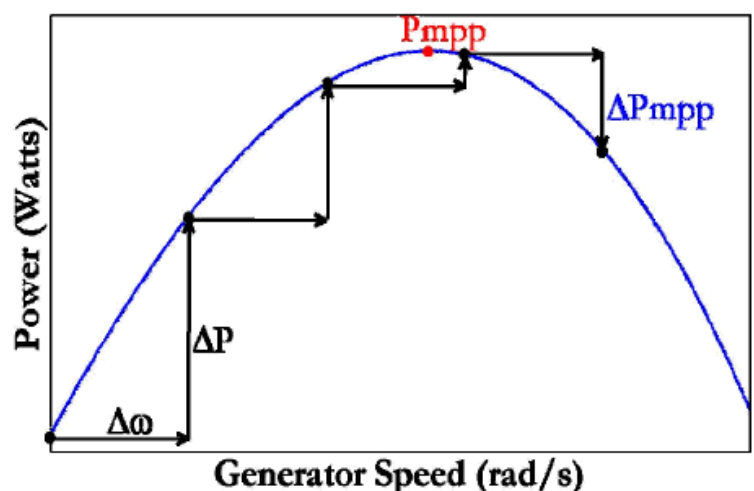

Figure 12: HCS Overshoot [19]

Smaller perturbations will increase the control efficiency but will decrease the convergence speed which is not ideal for rapidly varying wind conditions. The control algorithm efficiency can be expressed as follows 


$$
\eta_{m p p t}=\left(1-\frac{\Delta P_{m p p t}}{P_{m p p t}}\right) \times 100 \%
$$

Where $\Delta P_{m p p t}$ is the oscillation around the maximum power point, $P_{m p p t}$.

Another problem with the HCS is that changing wind conditions may cause the perturbation direction to be incorrect [19]. A perturbation in a certain direction may result in an increase in power but the increase is only because of a wind change. This will set the search in the wrong direction as illustrated in Figure 13.

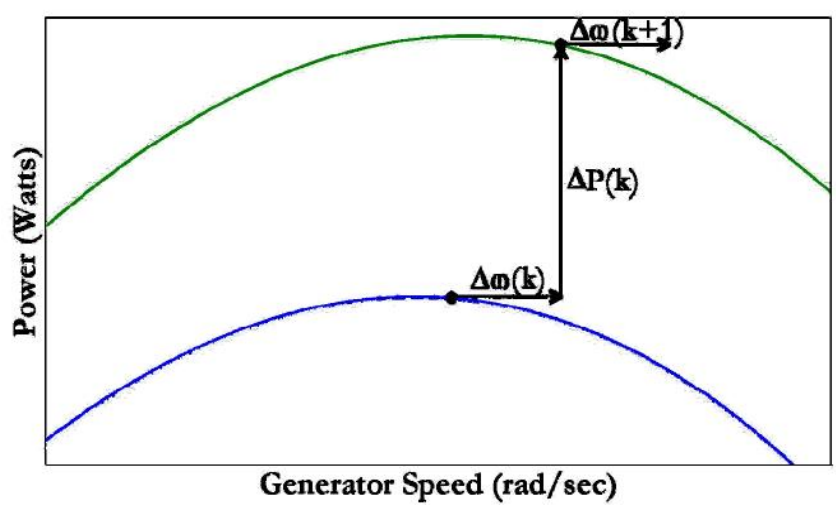

Figure 13: Direction Error for a Change in Wind Speed [19]

In addition, this method will not work correctly with large wind turbines due to the large inertia associated with the larger mechanical components of the system. The large inertia will not allow the speed to be changed quickly enough for this control to be efficient.

The HCS is a very useful general solution to the MPPT problem. There has been research into overcoming some of the problems associated with this technique. To increase the speed of convergence of the algorithm, a technique that uses memory can be used in conjunction with an anemometer [20].

\subsection{Turbine Emulator}

In order to emulate wind accurately, the phenomena that wind turbines experience need to be understood. Wind turbulence is an important concept as it can influence the performance of the turbine substantially. Consequently a method for implementing turbulence in the turbine emulator is explored in this section. Wind turbines also experience periodic power fluctuations that occur at a frequency of three times the rotational 
frequency. The fluctuation in power is due to torque pulsations that occur at the same moment. It is important to note that the converter control of a grid connected system might be affected by the pulsations. There are two main periodic processes that have the greatest effect on torque pulsations namely wind shear and tower shadow. The torque pulsations can be equated to changes in wind speed which are then modified into a normalised torque reference in order to be implemented in the turbine emulator.

\subsubsection{Wind Turbulence}

Wind profiles can be obtained from measurement over a long time period or from mathematical models that try to recreate profiles accurately. There are two models that are used to reproduce wind profiles for simulation purposes, namely the Van der Hoven and the Von Karman models [21]. The Van der Hoven model is used for large band wind speed and does not accurately model wind turbulence because it generates the same turbulence magnitude for any mean wind velocity. Turbulence is of greater importance in this project as it will have greater demands on the control of the system and for this reason the Von Karman model is used to model the wind turbulence which accounts for changes in the mean wind velocity. In [21] the turbulence component is modeled using a shaping filter, which is represented by equation 2.13 , with white noise as the input.

$$
\begin{gathered}
H_{F}(s)=K_{F} \frac{\left(m_{1} T_{F} s+1\right)}{\left(T_{F} s+1\right)\left(m_{2} T_{F} s+1\right)}, m_{1}=0.4, m_{2}=0.25 \\
T_{F}=\frac{L}{v_{m}}
\end{gathered}
$$

$T_{F}$ is determined by equation 2.14 , where $L$ length of the turbulence and $v_{m}$ is the mean wind velocity. Equations 2.16 and 2.17 are used to calculate $K_{F}$ which is represented by equation 2.15 choosing $\Delta \omega$ to be $0.002 \mathrm{rad} / \mathrm{s}$ and $\omega_{s}$ to be $2 \mathrm{rad} / \mathrm{s}$ [21].

$$
\begin{gathered}
K_{F}=\sqrt{\frac{\pi}{T_{S} \Delta \omega S}} \\
S=\sum_{k=0}^{J} \frac{\left(m_{1} T_{F} k \Delta \omega\right)^{2}+1}{\left[\left(T_{F} k \Delta \omega\right)^{2}+1\right]\left[\left(m_{2} T_{F} k \Delta \omega\right)^{2}+1\right]}
\end{gathered}
$$




$$
J=\frac{\omega_{s}}{2 \Delta \omega}
$$

The standard deviation of the turbulence $\sigma_{v}$ is calculated using equation 2.19 , where $k_{\sigma, v}$ is the slope of the turbulence, and is used to determine the instant wind velocity $v(t)$ in equation 2.18. $k_{\sigma, v}$ has a minimum value of 0.1 for open sea and increases to a value of 0.25 for developed areas [21].

$$
\begin{gathered}
v(t)=v_{m}+\sigma_{v} v_{t} \\
\sigma_{v}=k_{\sigma, v} v_{m}
\end{gathered}
$$

The von Karman model provides a very flexible algorithm for wind turbulence allowing testing of a wide range of sites and is ideal for an experimental laboratory setup.

\subsubsection{Wind Shear}

The strength of wind usually increases with height and this affects the torque that a set of blades can exert depending on their position. When a blade is pointing directly upwards, it will experience the greatest amount of wind and conversely it will experience the least amount of wind when it is pointing directly downwards. A three blade turbine will therefore experience three pulsations during one rotation as the blades move through the maximum and minimum wind areas. Wind shear can be expressed as:

$$
v_{e q_{w s}}=V_{h}\left[\frac{\alpha(\alpha-1)}{8}\left(\frac{r}{h}\right)^{2}+\frac{\alpha(\alpha-1)(\alpha-2)}{60}\left(\frac{r}{h}\right)^{3} \cos 3 \theta\right]
$$

in terms of wind speed, where $V_{h}$ is the wind speed at the hub height $h, r$ is the radius of the turbine, $\alpha$ is the empirical wind shear exponent and $\theta$ is the azimuthal angle [22]. $\alpha$ is dependent on the terrain type having a value of 0.1 over open water and 0.3 in towns [23]. The height of the tower will also affect $\alpha$ as the wind will be less turbulent at greater heights. 


\subsubsection{Tower Shadow}

The second type of periodic process, tower shadow, occurs when the blade passes the turbine tower. In this region the blades experience a lower wind velocity. This phenomenon occurs because the wind needs to change direction in order to pass the tower reducing net wind speed that the blade experiences.

$$
\begin{gathered}
v_{e q_{t s}}=\frac{m V_{h}}{3 R^{2}} \sum_{b=1}^{3}\left[\frac{a^{2}}{\sin ^{2} \theta_{b}} \ln \left(\frac{r^{2} \sin ^{2} \theta_{b}}{x^{2}}+1\right)-\frac{2 a^{2} r^{2}}{r^{2} \sin ^{2} \theta_{b}+x^{2}}\right] \\
\theta_{1}=\theta, \theta_{2}=\theta_{1}+\frac{2 \pi}{3}, \theta_{3}=\theta_{1}+\frac{4 \pi}{3}
\end{gathered}
$$

$V_{h}$ is the wind speed at hub height $h, r$ the radius of the blades and $x$ is the distance of the blades from the tower. $m$ is calculated using equation 2.22 where $h$ is the tower height [22].

$$
m=1+\frac{\alpha(\alpha-1) r}{8 h^{2}}
$$

\subsection{Power Quality and Grid Code}

The voltages on the distribution network are not always perfectly balanced and can have an adverse effect on the control of the turbine if not dealt with correctly. There are grid codes that exist and need to be complied with, demanding certain responses from a turbine under both balanced and unbalanced situations. The South African grid code requires wind turbines to provide reactive power in the case of low voltage situations as illustrated in Figure 15. Additionally, the turbine is expected to stay connected to the grid under the low voltage conditions illustrated in Figure 14 [24]. The effects of low voltage conditions need to be understood in order to provide solutions for the control system algorithm and are investigated in chapter 3. 


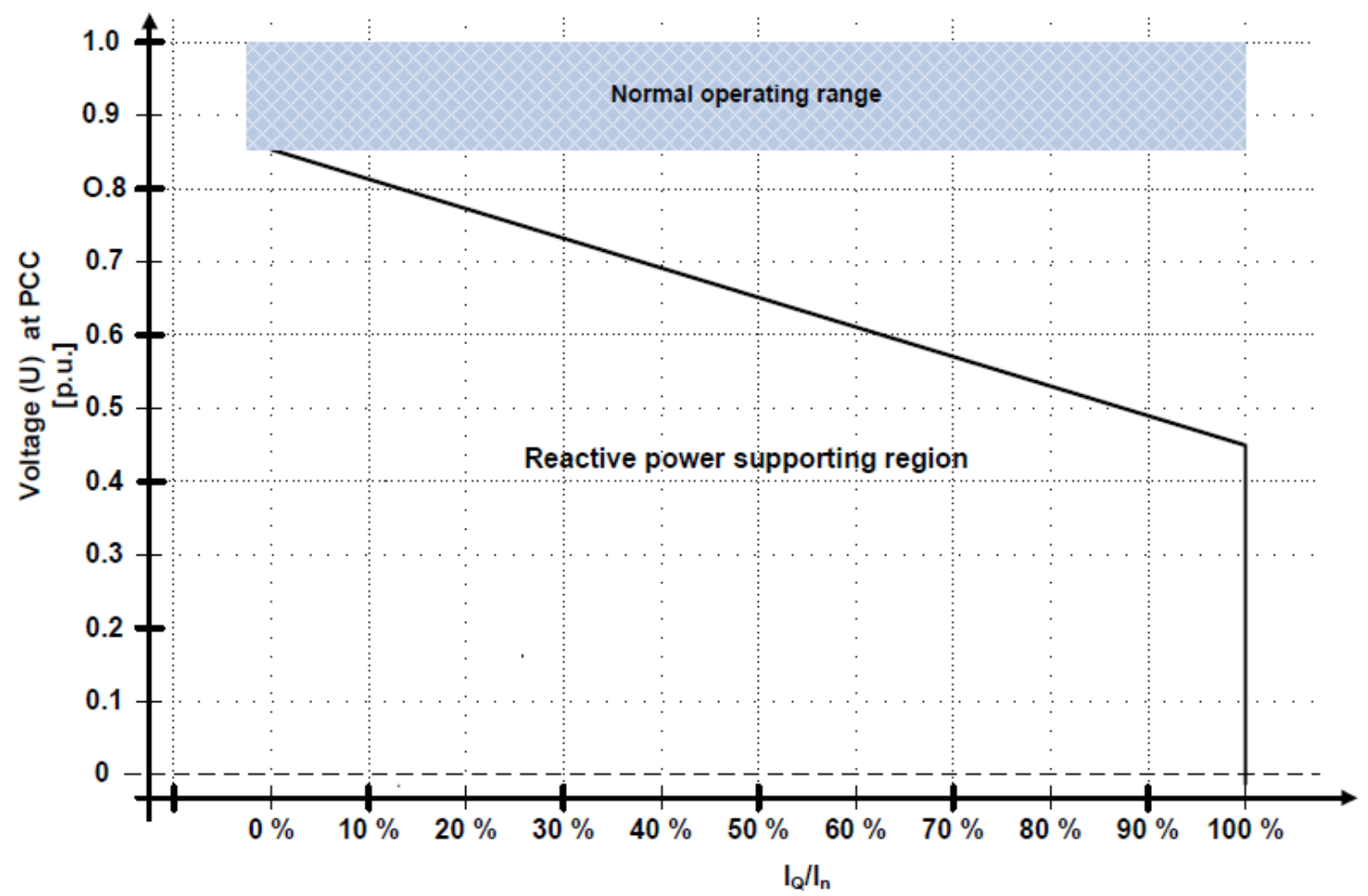

Figure 15: Requirements for Reactive Power Support During Voltage Drops [24]

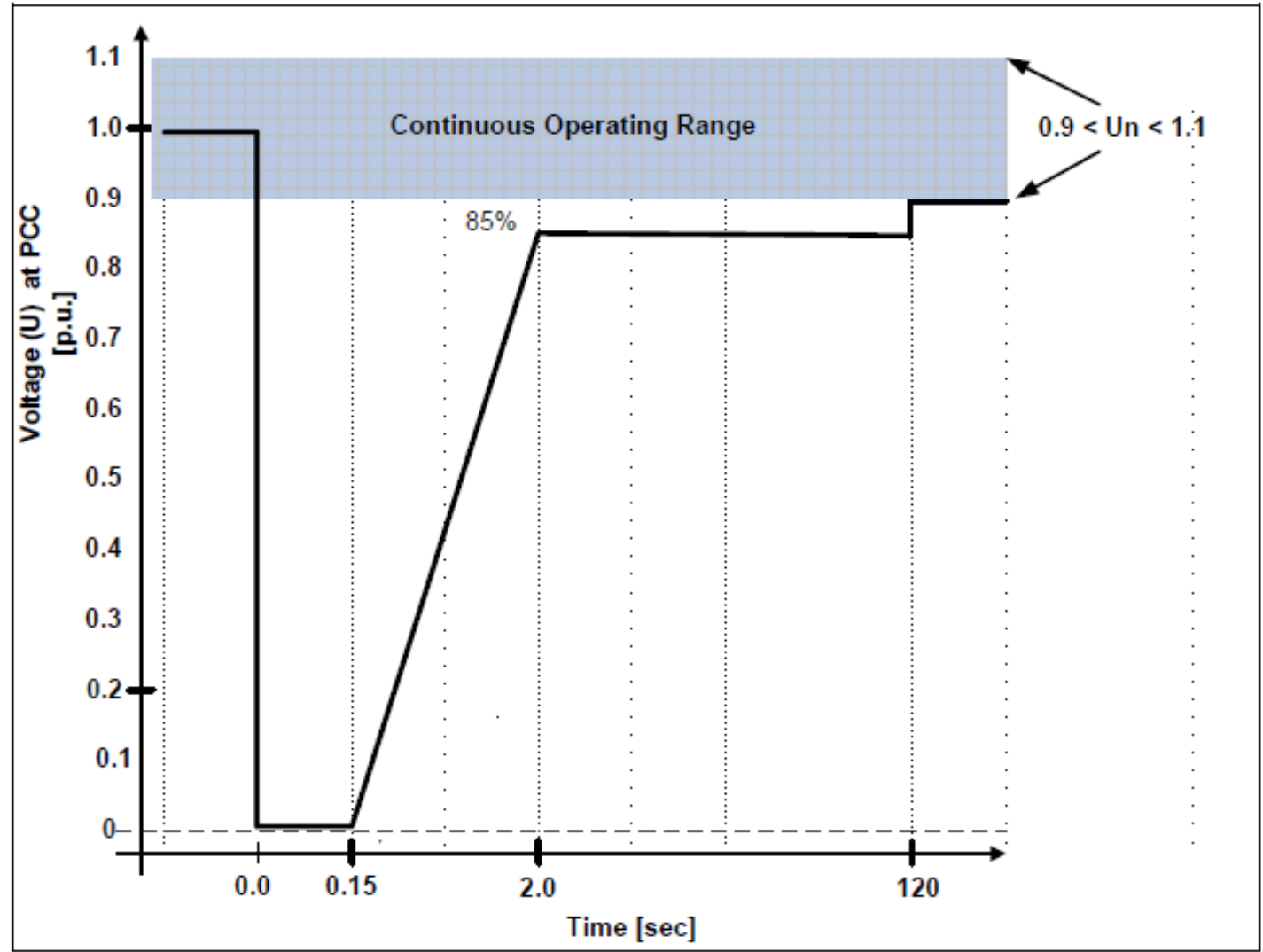

Figure 14: Low Voltage Ride Through Capability for a Wind Turbine [24] 
Voltage dips can be classified into balanced and unbalanced dips. A balanced dip occurs when all three phases sag without phase angle shifts. This type of a dip is classified as type A. Unbalanced dips fall into two categories, single phase and double phase. The cause of each type of dip is a combination of either a phase-ground or phase-phase fault occurring in either a star or delta connected system. Figure 16 shows the dip classification while Table 4 illustrates the cause of each type of dip. [25] For the purposes of this project only the normal operating range in figure 24 will be considered and it is assumed that the currents in the range are required to be balanced.
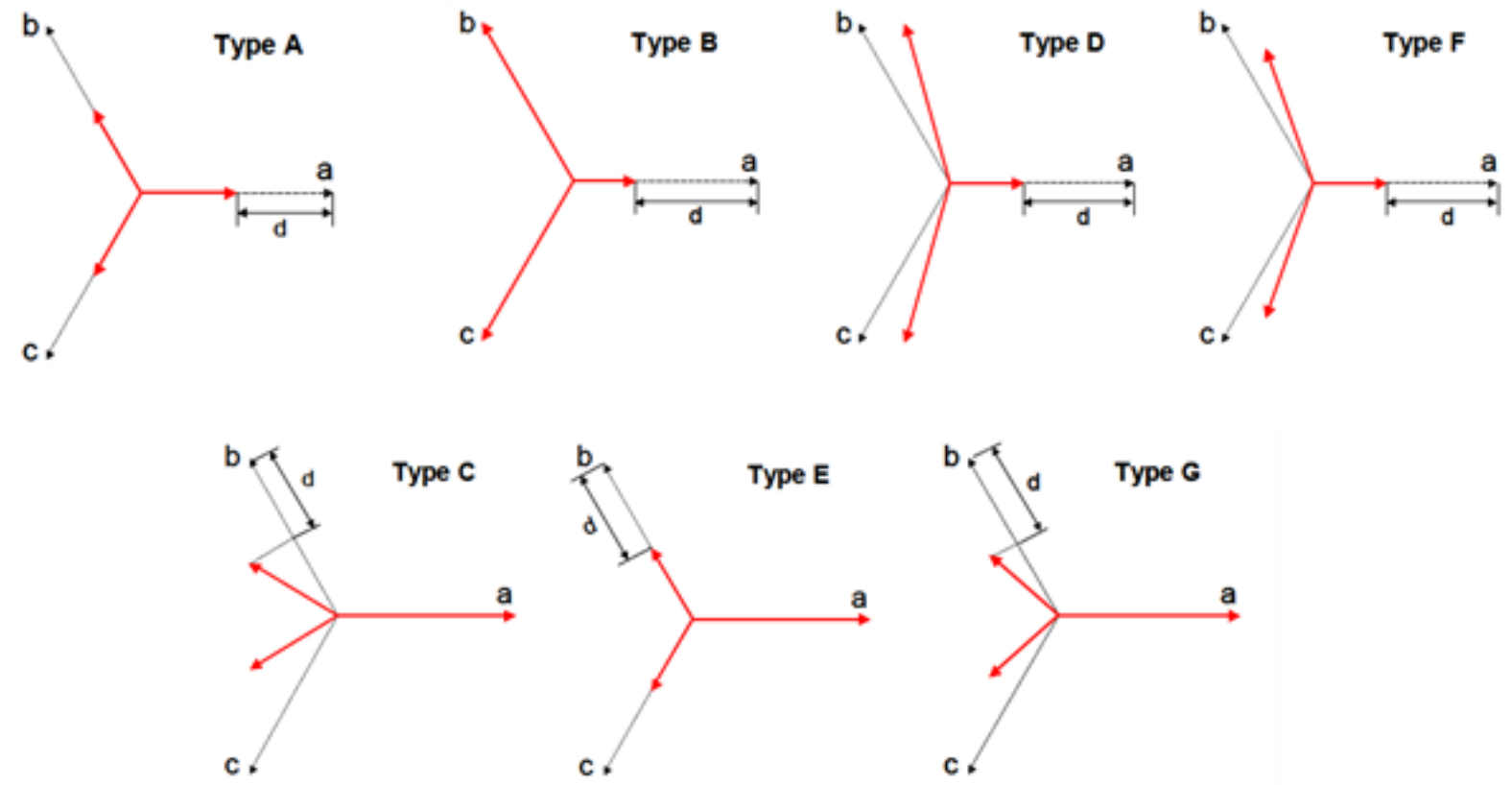

Figure 16: Dip Types [25] 
Table 4: Dip Types and Causes [26]

\begin{tabular}{|l|c|c|}
\hline \multicolumn{1}{|c}{ Fault Type } & \multicolumn{1}{c|}{$\begin{array}{c}\text { Star- } \\
\text { Connected }\end{array}$} & $\begin{array}{c}\text { Delta- } \\
\text { Connected }\end{array}$ \\
\hline Three Phase & Type A & Type A \\
\hline Phase-to-Phase & Type C & Type D \\
\hline Single-Phase & Type B & Type C \\
\hline Two-Phase-to-Ground & Type E & Type F \\
\hline
\end{tabular}

\subsection{Conclusion}

This chapter reviewed the different WECS topologies and built an understanding of how wind energy is converted into electrical energy. Various MPPT techniques were investigated as well as the possible power quality conditions that can occur on the grid side and the turbine side. Additionally, the grid code's expectation of a WECS during the non-ideal conditions are also investigated. In this project a PMSG was studied using the Power-Speed curve for MPPT. Only normal grid operating conditions are considered for testing the system. 


\section{CHAPTER 3}

\section{FULL CONVERTER CONTROL}

This chapter looks at the control methods used for the back-to-back converter. Initially, Vector Control (VC) was used to control both sides of the back-to-back converter. VC is implemented in the synchronous reference frame and the relevant transformations are presented in this chapter. However, the VC had problems operating under unbalanced grid conditions. When grid unbalance is explored, a modified version of VC is introduced, which is called dual vector current control.

\subsection{Reference Frame Theory}

Various reference frames exist that can represent electrical space-phasor quantities. The synchronous reference frame is used to implement FOC and the transformations that lead to it are investigated in this section. Although current is referred to in the following examples, the methods can be applied to any space-phasor quantities.

\subsubsection{Natural reference frame}

The currents of a balanced three-phase system can be represented as space-phasors that have a length equal to their instantaneous magnitude along axes that are displaced by $120^{\circ}$ in space. Space-phasors obtained in this manner can be combined to form a resultant phasor component which has a magnitude of $1.5 I_{m}$, where $I_{m}$ is the peak value of the current phasors, and rotates at a constant angular frequency determined by the frequency of the phase currents. The resultant phasor is normalised to make it magnitude invariant. It will then have a magnitude that is equal to the peak of the phasor currents which is ideal for current control methods. The definition of the resultant space-phasor in terms of the phase current is given by equation 3.1 as:

$$
i_{s}=\frac{2}{3}\left(i_{a}+i_{b} e^{j \frac{2 \pi}{3}}+i_{c} e^{j \frac{4 \pi}{3}}\right)
$$




\subsubsection{Stationary reference frame}

This reference frame represents the current phasor $I_{S}$ on a set of orthogonal axes. The real axis $\alpha$ is chosen to be aligned with the phase $A$ current phasor and the imaginary $\beta$ axis lies in quadrature. The transformation from the natural reference frame to the $\alpha \beta$ reference frame is called the Clarke transform and is presented in equation 3.2.

$$
\left[\begin{array}{l}
i_{\alpha} \\
i_{\beta}
\end{array}\right]=\frac{2}{3}\left[\begin{array}{ccc}
1 & -\frac{1}{2} & -\frac{1}{2} \\
0 & \frac{\sqrt{3}}{2} & -\frac{\sqrt{3}}{2}
\end{array}\right]\left[\begin{array}{l}
i_{a} \\
i_{b} \\
i_{c}
\end{array}\right]
$$

\subsubsection{Synchronous Reference Frame}

The stationary reference frame components are still sinusoidal in nature which can complicate control somewhat. These components are further projected onto a new set of axes that rotates at the same angular frequency $(\omega)$ as the currents. The new reference frame is called the synchronous or rotating reference frame. The real d-axis is located at an angle $\theta=\omega t$ which is chosen to correspond with the pole axis of the rotor in machines and the grid voltage phasor in a grid-tied inverter. The imaginary q-axis is in quadrature with the $\mathrm{d}$-axis. The transformation from the $\alpha \beta$ reference frame to the $d q$ reference frame is achieved with the Parke transform which is presented in equation 3.3. All the reference frames discussed above are illustrated in Figure 17.

$$
\left[\begin{array}{l}
i_{d} \\
i_{q}
\end{array}\right]=\left[\begin{array}{cc}
\cos \theta & -\sin \theta \\
\sin \theta & \cos \theta
\end{array}\right]\left[\begin{array}{l}
i_{\alpha} \\
i_{\beta}
\end{array}\right]
$$




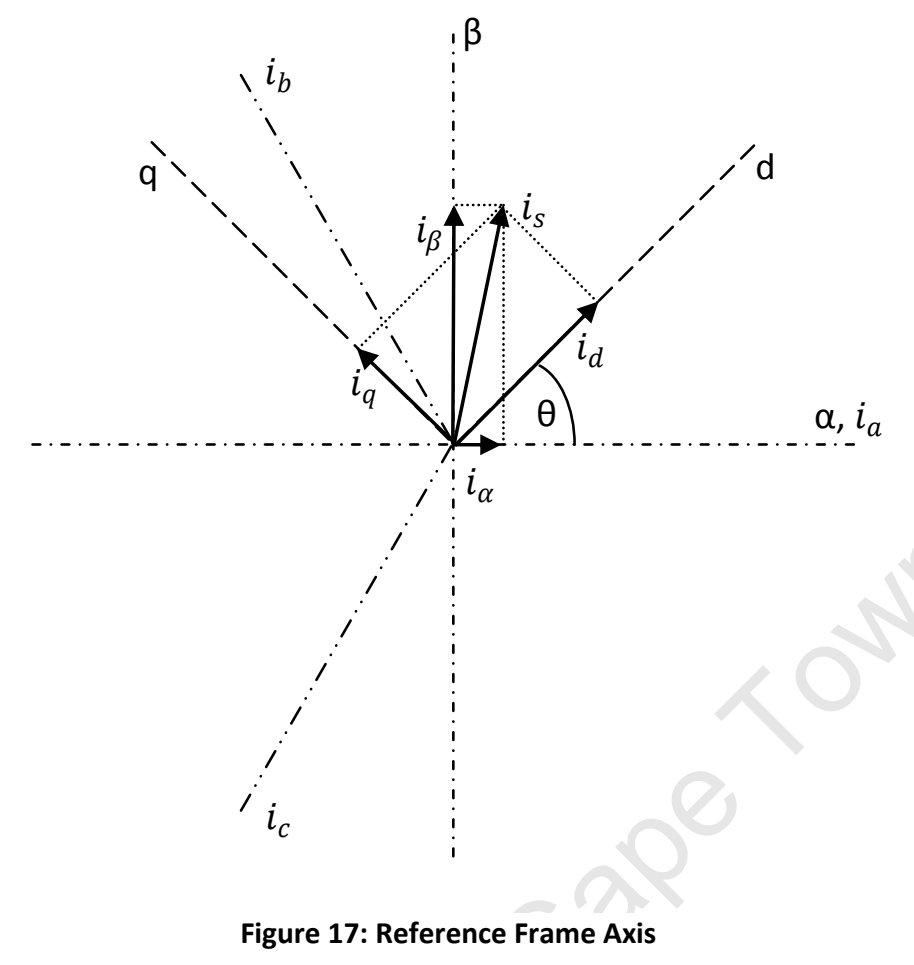

\subsection{Generator Control}

The generator used in this thesis is a 12 pole, $240 \mathrm{rpm}$, surface mounted permanent magnet machine, rated at $6 \mathrm{~kW}$. The generator is controlled using a MPPT technique and transfers the power to a DC Link. The position and speed of the generator are determined by an encoder, which is discussed further in section 6.3.2. To perform current control on the generator, the phase currents are monitored using LEM modules which are discussed further in section 6.3.1. All of the measured signals are sent to a DSpace kit for processing which produces the required PWM signals. Figure 18 illustrates the layout of the generator side control for the project. 


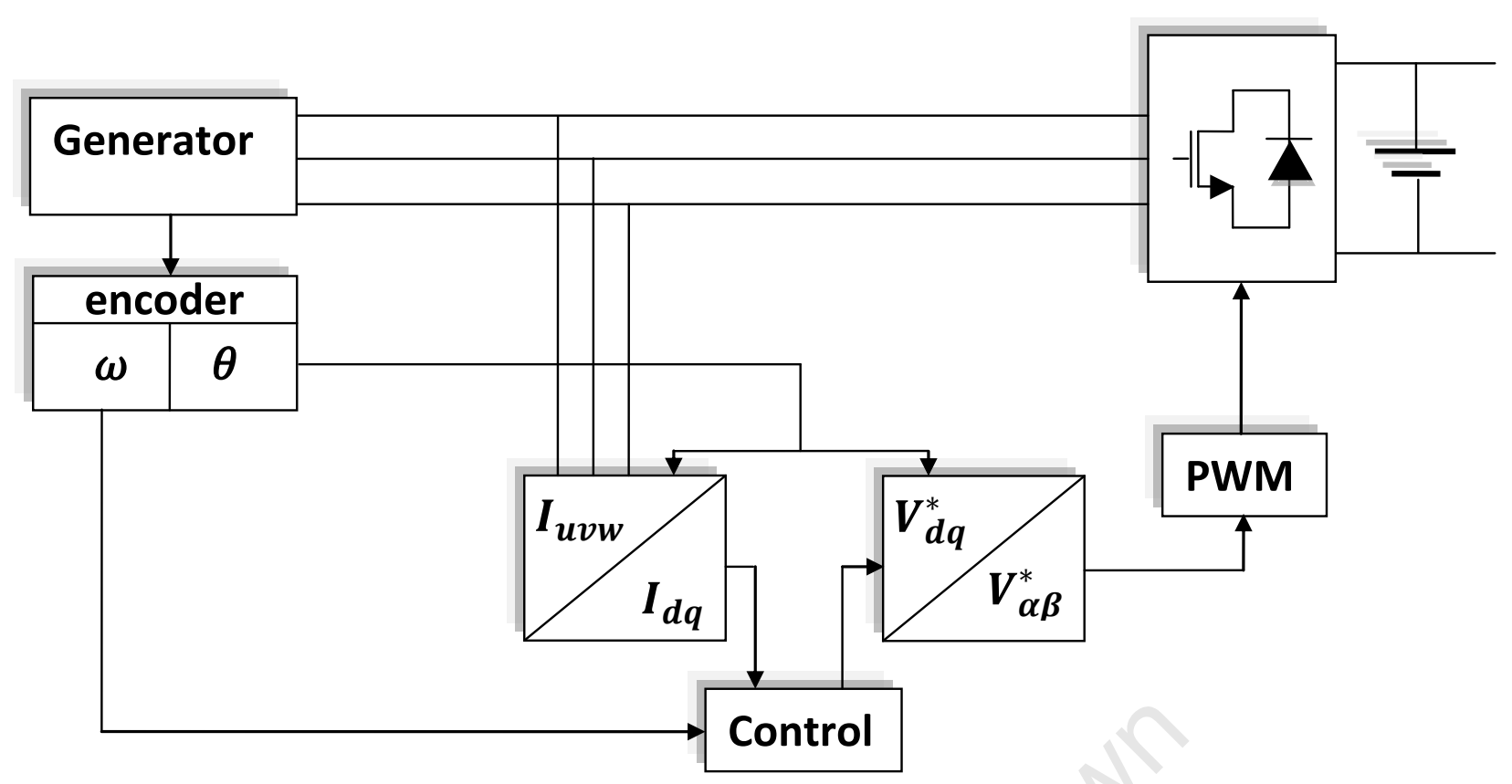

Figure 18: Generator Control Overview

$I_{u v w}$ are the generator phase currents and $I_{d q}$ are the corresponding currents in the synchronous reference frame. The generator-side control will be discussed in detail in this section.

\subsubsection{DQ model of the PMSG}

The $d q$ model represents the model of the generator in the $d q$ reference frame. In order to develop a generalised model and control technique for the machine, an interior PM synchronous generator (IPMSG) is considered because a surface mounted PM machine is a special case of the IPMSG model. The $d$ and $q$-axis equivalent circuit models of an IPMSM are shown in Figure 19 and Figure 20.

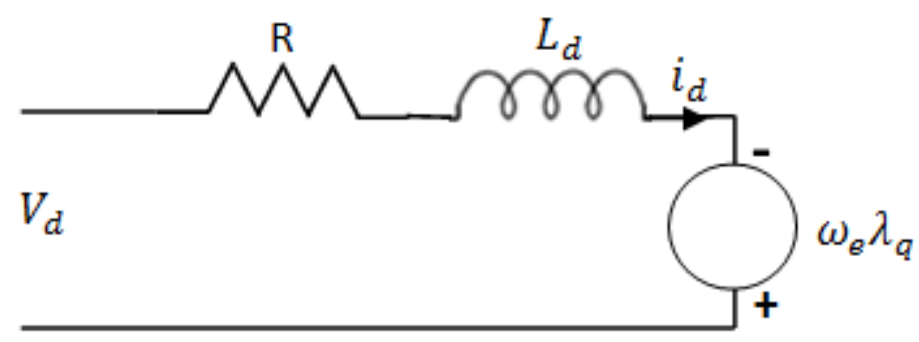

Figure 19: d-axis Machine Model 


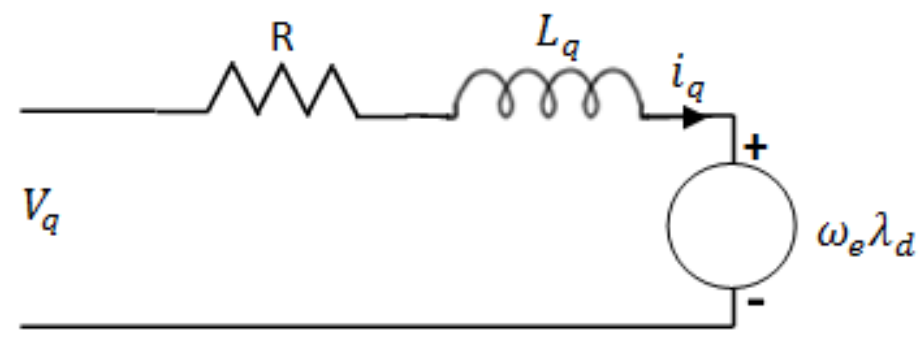

Figure 20: q-axis Machine Model

The total $d q$ flux linkages for the machine can be expressed as follows

$$
\begin{gathered}
\lambda_{q}=L_{q} i_{q} \\
\lambda_{d}=L_{d} i_{d}+\lambda_{p m}
\end{gathered}
$$

There are two types of component voltages induced in the $d q$ axis coils, namely, transformer and rotational emfs. Transformer emfs occur as a result of the rate of change of the total flux linkage in the respective coils and are represented by the terms 3.6 and 3.7 in the $d$ and q-axis circuits respectively.

$$
\begin{aligned}
& \text { d-axis: } \frac{d \lambda_{d}}{d t} \\
& \text { q-axis: } \frac{d \lambda_{q}}{d t}
\end{aligned}
$$

The $d q$ rotational emfs are induced as a result of the relative movement between the stationary stator windings and the total $d q$ fluxes in the machine. They are represented by equations 3.8 and 3.9 for the $d$ and $q$ axis respectively. 


$$
\text { d-axis: }-w \lambda_{q}
$$

q-axis: $+w \lambda_{d}$

The rotational emfs in Equations 3.8 and 3.9 introduce a cross coupling effect into the $d q$ axis voltage equations which are expressed in equations 3.10 and 3.11 .

$$
\begin{aligned}
& v_{d}=R i_{d}+\frac{d}{d t} \lambda_{d}-w \lambda_{q} \\
& v_{q}=R i_{q}+\frac{d}{d t} \lambda_{q}+w \lambda_{d}
\end{aligned}
$$

The cross-coupling effect can be eliminated for by subtracting terms 3.8 and 3.9 by means of the control algorithm. The voltage equations can therefore be considered decoupled from one another.

The electromagnetic torque developed can be expressed as 3.12 and further simplified to 3.13 for a surface mounted machine where $L_{d}=L_{q}$.

$$
\begin{gathered}
T_{e}=\frac{3}{2} p\left[\lambda_{p m} i_{q}+\left(L_{d}-L_{q}\right) i_{d} i_{q}\right] \\
T_{e}=\frac{3}{2} p \lambda_{p m} i_{q}
\end{gathered}
$$


A torque balance equation including the load torque can be expressed as 3.14 .

$$
T_{e}-T_{\text {load }}=\frac{J}{p} \frac{d w}{d t}+B \omega
$$

The control of the generator was designed based on these equations.

\subsubsection{Control of the Generator}

The generator control regulates the stator current and hence the torque of the generator. Vector control is used along with Space Vector Pulse Width Modulation (SVPWM) as a switching scheme. The full bridge converter is controlled using a DSpace kit which acts as an interface between the switches and the computer. Figure 21 is a detailed diagram of the generator-side control.

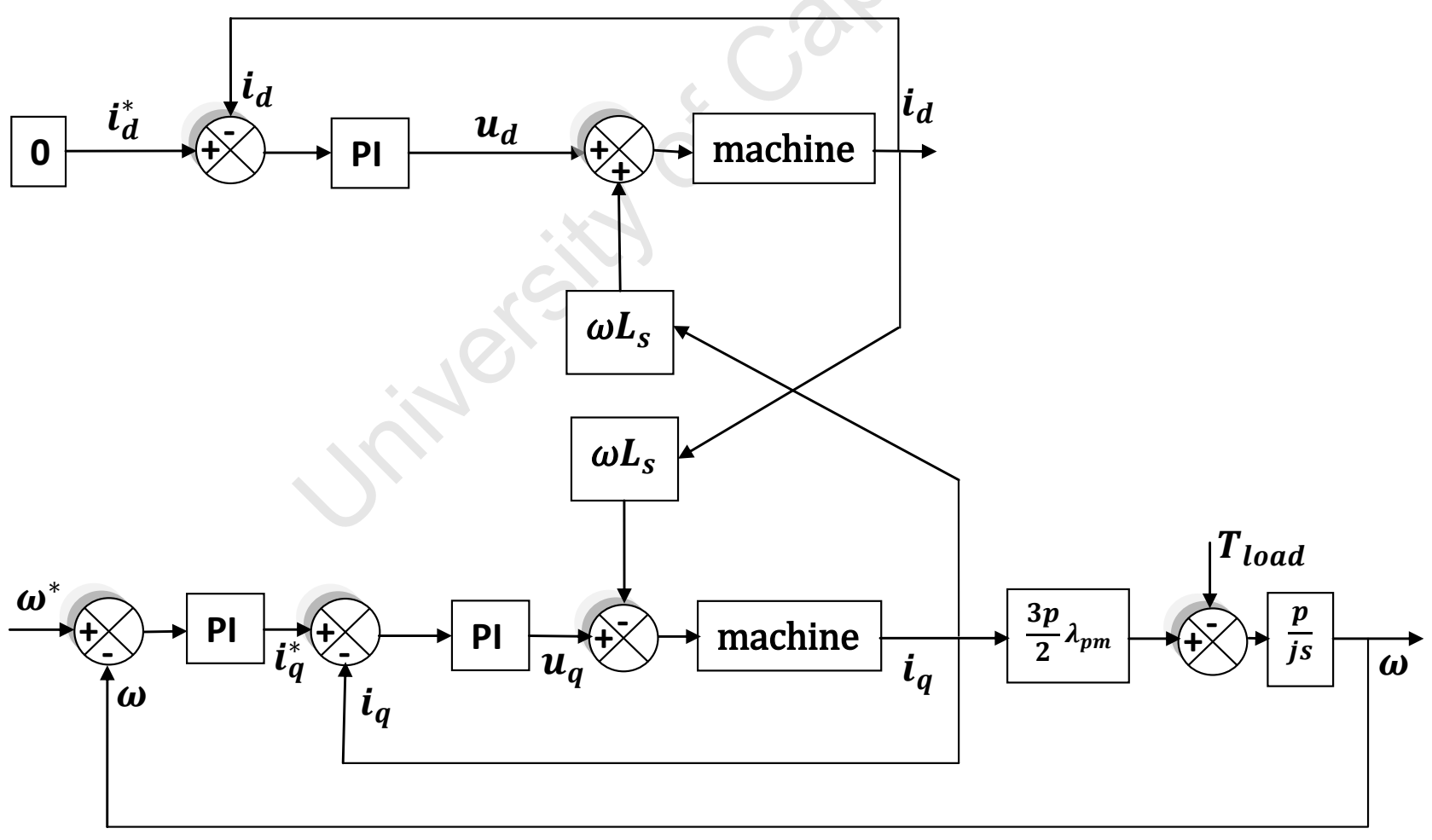

Figure 21: Detailed Generator Control Algorithm with MTA implemented 
For this thesis the Maximum Torque per Ampere (MTA) method is used to control the surface PM machine. This method maximizes the amount of torque produced per ampere of stator current. With reference to equation $3.13, L_{d}=L_{q}$ in a surface PM machine, which means that $i_{d}$ does not contribute towards torque production. It is therefore set to zero in order to minimise the stator current. The major advantage of the MTA technique is that it minimises the copper losses which are calculated by equation 3.15 .

$$
W_{c u}=\frac{3}{2} R\left[i_{d}^{2}+i_{q}^{2}\right]
$$

\subsection{Grid-Side and Control}

The grid-side consists of a full bridge converter connected to the grid using a filter. Selecting the type of filter as well as the component values is critical for reducing the harmonic content in the grid currents and will be discussed further in section 3.5.2. The grid control requires the grid voltages and currents as well as the DC link Voltage to be monitored. Figure 22 provides an overview of the grid side control.

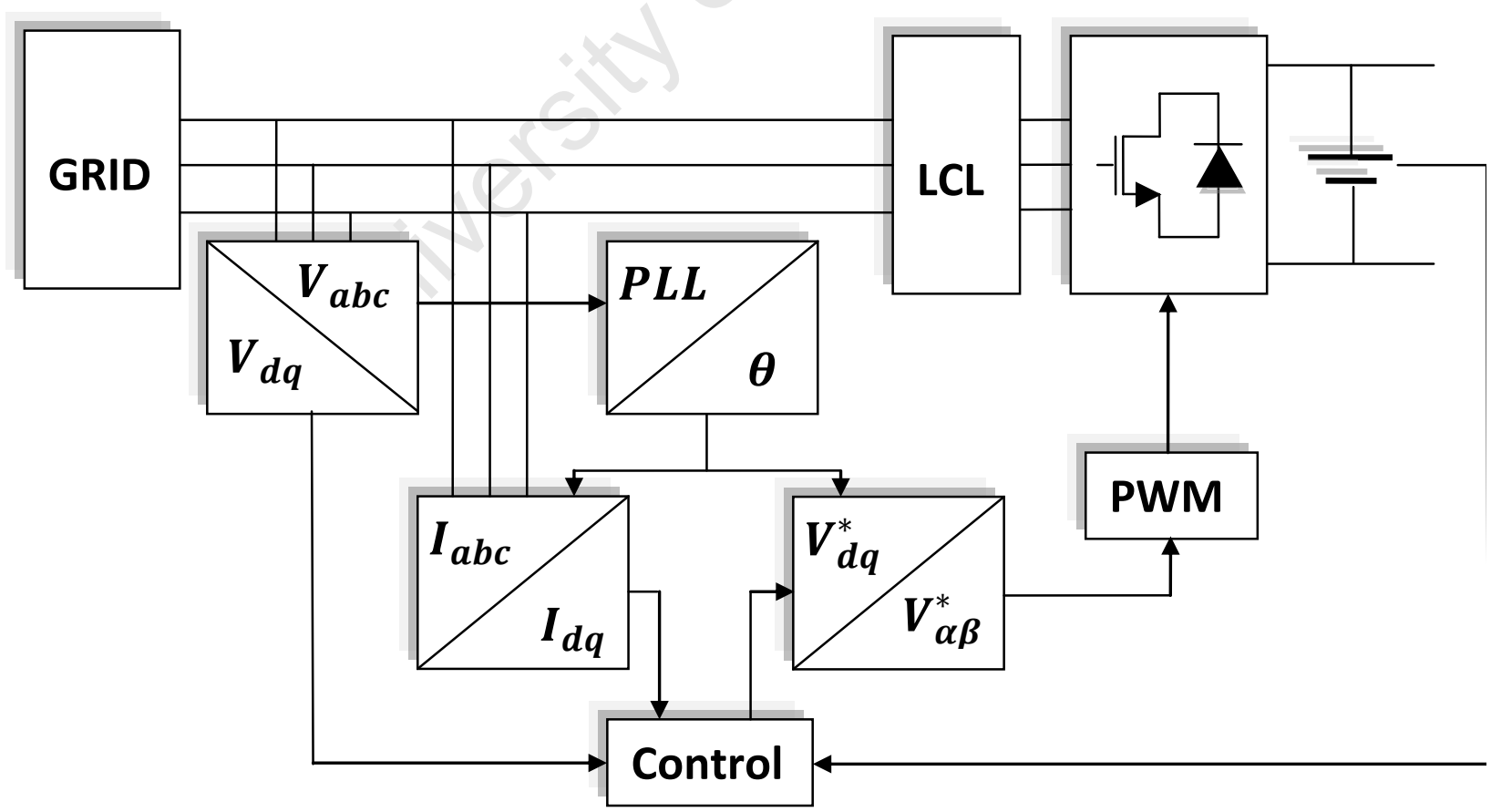

Figure 22: Grid Side Control Overview 
The converter is controlled in the same manner as the generator-side except that Unity Power Factor (UPF) control is embedded within the vector control.

\subsubsection{DQ Model of Grid Side}

As with the generator-side converter, the model of the grid-side needs to be understood in order for the control to be implemented. Equations 3.16 and 3.17 present the voltage equations of the grid side. The equations resemble the generator model but have to take into account both the converter and grid voltages.

$$
\begin{aligned}
& V_{\text {dgrid }}=R_{T} i_{d}+L_{T} \Delta i_{d}+V_{d c o n v}-w L_{T} i_{q} \\
& V_{\text {qgrid }}=R_{T} i_{q}+L_{T} \Delta i_{q}+V_{q c o n v}+w L_{T} i_{d}
\end{aligned}
$$

Where $R_{T}$ is the total parasitic resistance of the filter components and $L_{T}$ is the total inductance of the LCL filter. The cross coupling terms that are given by equations 3.18 and 3.19 represent the voltage across the filter inductors. They can once again be compensated for in the control algorithm to decouple the $d$ and $q$ axis circuits, in order to simplifying the control design.

$$
\begin{aligned}
& \text { d-axis: }-w L_{T} i_{q} \\
& \text { q-axis: }+w L_{T} i_{d}
\end{aligned}
$$

The above equations govern the design of the control system for the grid-side converter.

\subsubsection{Control of Grid Side}

The grid side of the converter is used to regulate the DC link voltage by transferring excess power to the grid. Similar to the generator side control, VC is used along with SVPWM to control the converter. The grid control is synchronized with the grid waveforms by means of 
a Phase-Locked Loop (PLL). The active and reactive powers transferred to the grid are expressed in equations 3.20 and 3.21 .

$$
\begin{aligned}
& P_{\text {grid }}=\frac{3}{2} v_{d} i_{d} \\
& Q_{\text {grid }}=\frac{3}{2} v_{q} i_{q}
\end{aligned}
$$

Unity Power Factor (UPF) operation is implemented within the control scheme by setting the reactive power to zero. This can be deduced from equation 3.21 and is achieved when the quadrature current $i_{q}$ is kept at zero. Figure 23 illustrates the VC of the grid side converter.

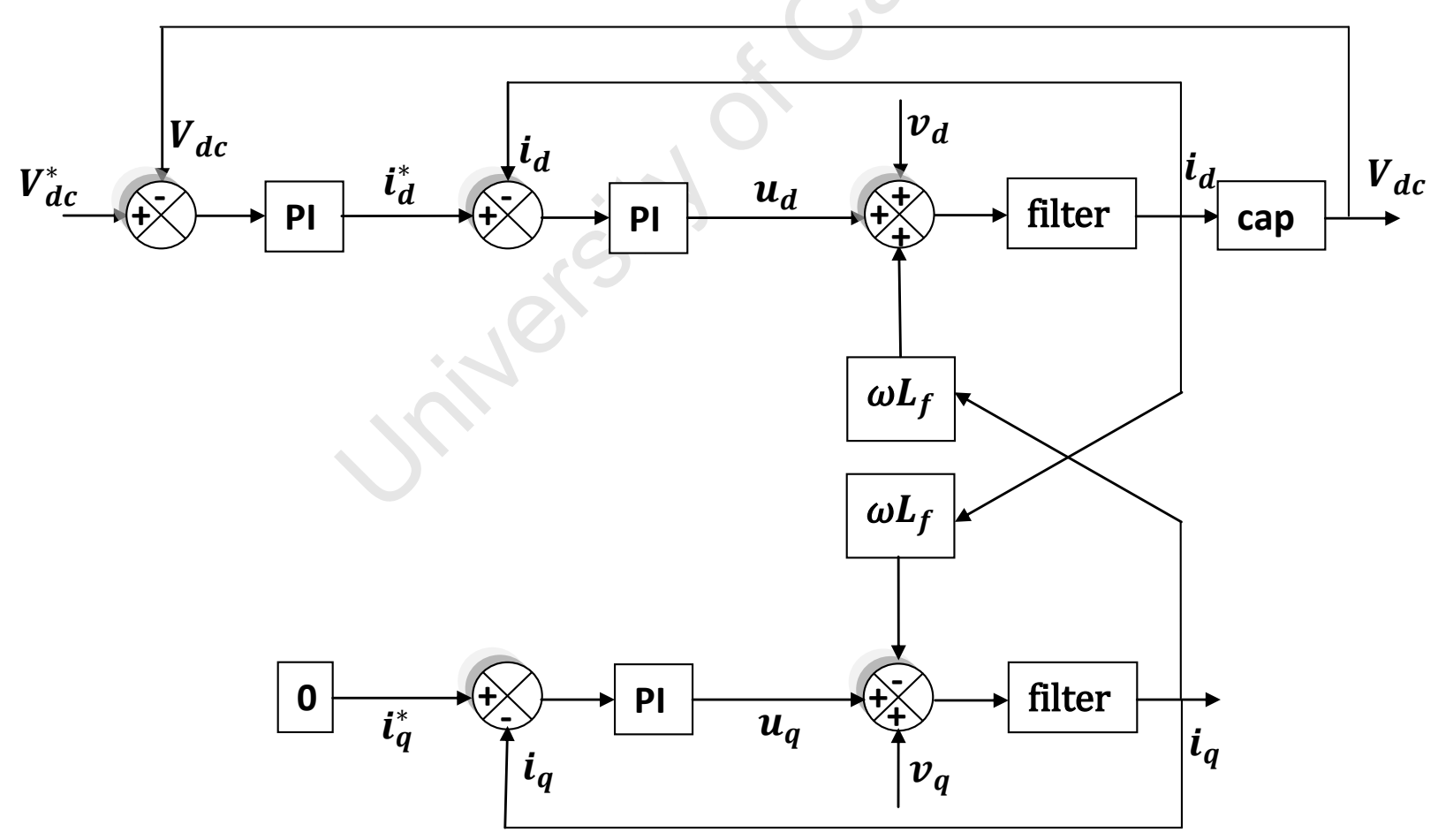

Figure 23: Detailed Control Algorithm for VOC with UPF control

\subsection{LCL Filter}

Voltage source converters are capable of producing currents with a total harmonic distortion of below $5 \%$. The switches of the converter switch at high frequencies, up to tens 
of $\mathrm{KHz}$, thereby introducing high frequency harmonics which are undesirable and considered to be responsible for current ripple. In the past, L-type filters have been used in between the grid and inverter to reduce the current harmonics. They are reasonable options for low power systems because the value of the inductance can be kept relatively low to achieve an acceptable amount of current harmonic attenuation. Furthermore, there are very few design options to consider which makes them easy to implement in a system. However, as the power of the system increases, the inductance value and hence, the inductor will both become larger and therefore more expensive. A larger inductance will also increase the voltage drop across the filter, increasing the system losses. Under these conditions it is feasible to use a LCL filter to reduce the cost and size of the system. A LCL filter has a much larger current harmonic attenuation at higher frequencies for the same inductance value as a L-type filter. This is ideal for reducing the high frequency switching harmonics and allows the filter to be smaller for the same attenuation. A LCL filter does however require more consideration than a L-type filter and characteristics such as ripple current, filter size, resonance, switching ripple attenuation and reactive power absorbed need to be analyzed whilst designing the filter [27] [28] [29].

Equations 3.22 and 3.23 show the transfer functions of a L-type filter and LCL filter respectively. The parasitic resistances are ignored when comparing the two filter types since they are much smaller in magnitude compared to the impedance of the inductors, but must be included when the system control is designed.

$$
\begin{gathered}
H_{L}=\frac{1}{s L_{T}} \\
H_{L C L}=\frac{1}{s^{3} L_{1} L_{2} C_{2}+s\left(L_{1}+L_{2}\right)}
\end{gathered}
$$

Figure 24 includes the bode plots of a $L$ and a $L C L$ filter with the same total inductance. 


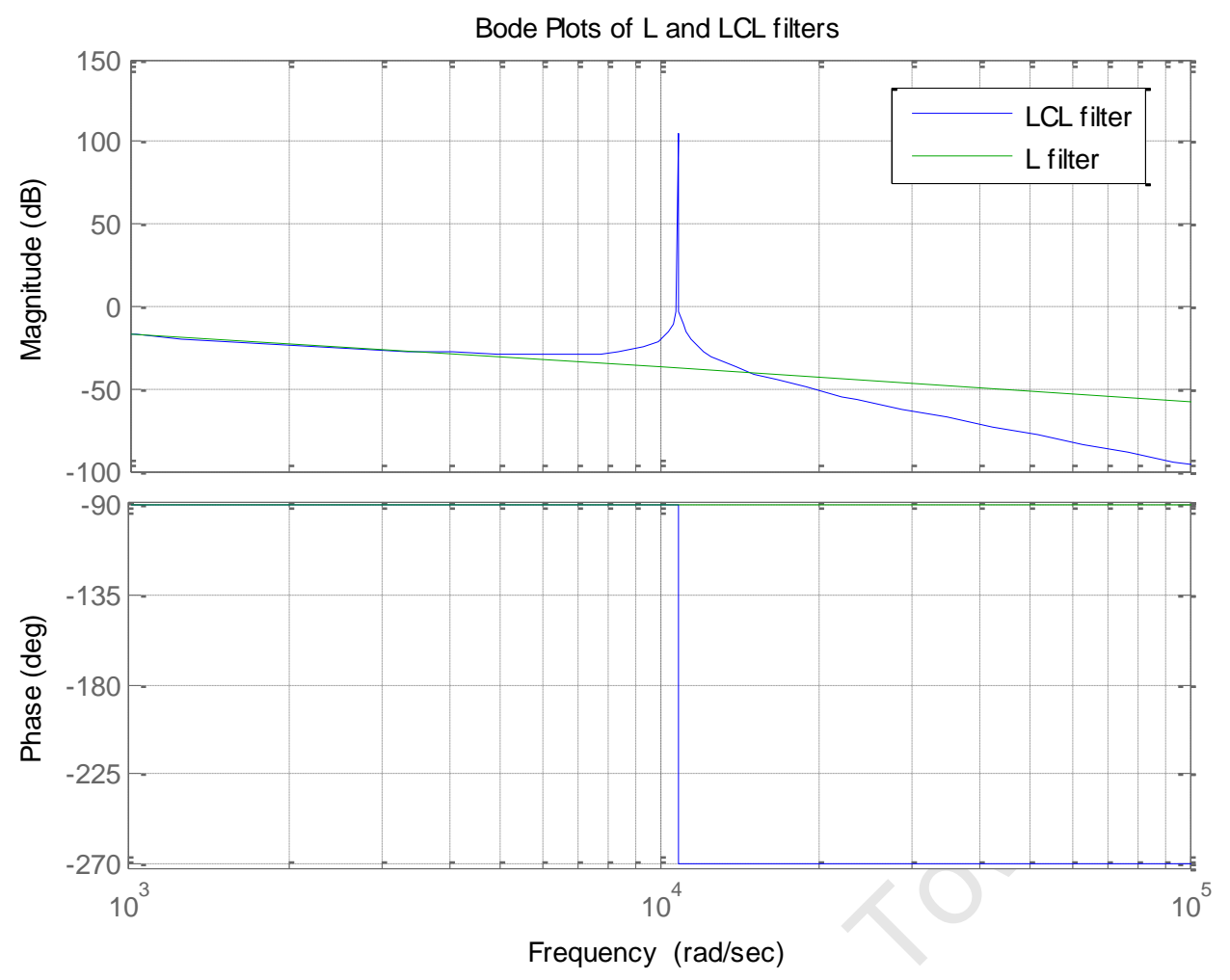

Figure 24: L and LCL Filter Bode Plots

Figure 24 shows that for the same inductance, the LCL filter attenuation is superior at higher frequencies. It also shows that an LCL filter introduces resonance to the system. This resonance can lead to the system being unstable and needs to be damped in order to increase stability. Two methods of damping exist, namely the passive and active methods. Passive damping techniques are simple to implement but at the expense of increased filter losses, whilst active damping techniques have no power losses but at the cost of increased control complexity.

\subsubsection{Passive Damping}

A resistor can be connected either in series or parallel with the filter inductor or capacitor to damp the oscillations caused by the filter resonance and is illustrated in Figure 25. 


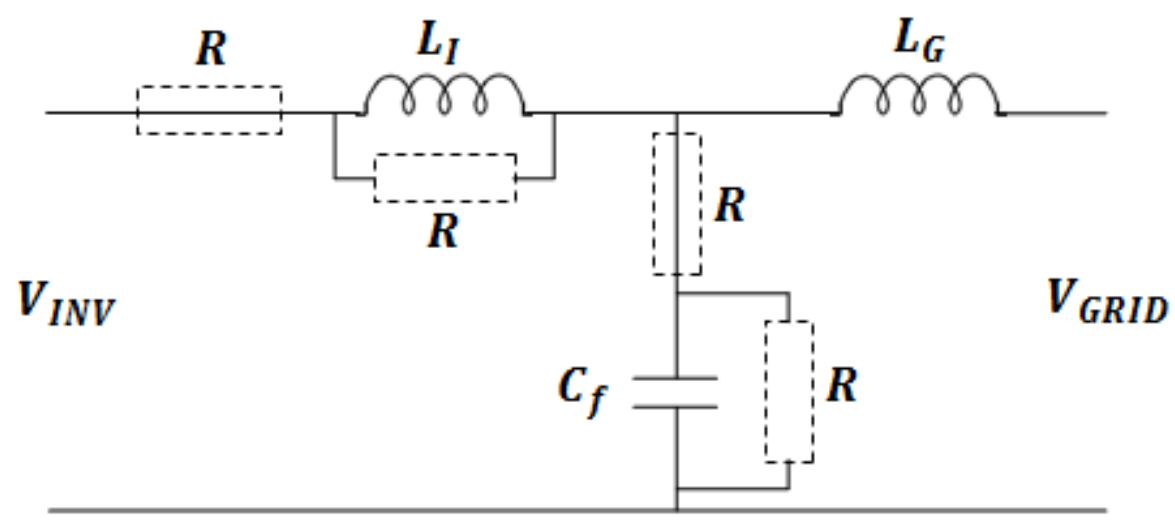

Figure 25: Passive Damping Resistor Positions

The most common methods are in series or parallel with the filter capacitor. In the case when the damping resistor is in series with the capacitor, an increase in resistance results in an increase in damping and therefore improved stability. The total power lost through the addition of the damping resistors in series with the filter capacitor is expressed in equation 3.24 .

$$
P_{\text {series }}=3 R_{d} \sum_{h}\left[i(h)-i_{g}(h)\right]^{2}
$$

Where $i(h)$ and $i_{g}(h)$ are the $h$-order harmonics of the converter and grid side currents respectively [27]. A resistor connected in parallel with the filter capacitor incurs power losses according to equation 3.25. The power loss decreases as the damping resistance increases. This method is used in utility-scale converters because if the grid fails, the filter capacitor is able to discharge through the resistor [30][34].

$$
P_{\text {parallel }}=3 \frac{1}{R_{d}} \sum_{h}\left[u_{c}(h)\right]^{2}
$$

The selection of passive damping resistors is discussed further in chapter 4 .

\subsubsection{Active Damping}

The various active damping techniques that are found in literature are presented in this section. These techniques are vital to decreasing losses when damping is required. 


\subsubsection{Virtual resistor}

A virtual resistor can be connected in the same positions as the passive damping resistors. A virtual resistor that is connected in series with the capacitor requires the currents in the components to be monitored and then differentiated. The resulting signal is then injected into the converter current reference signal to compensate for the resonance. This method requires extra current sensors and the differentiation will amplify high frequency noise. Figure 26 illustrates the implementation of a series virtual resistor.

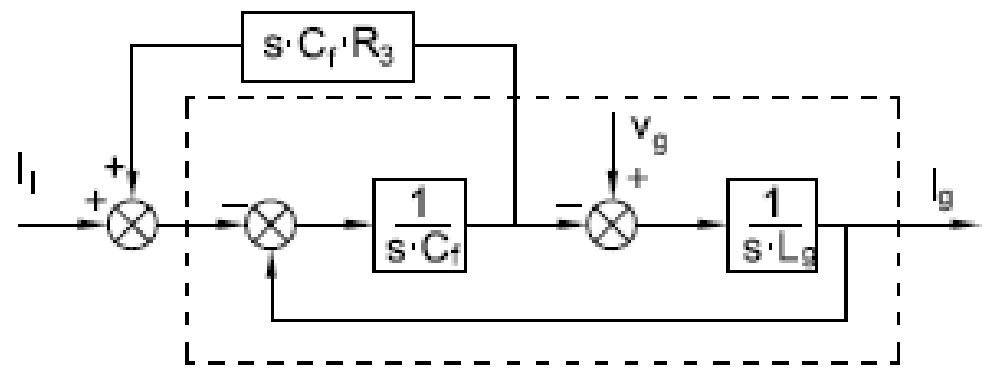

Figure 26: Block Diagram Implementation of Series Virtual Resistor [32]

Where $I_{i}$ and $I_{g}$ are the inverter and grid currents respectively and $R_{3}$ is the series resistor. If a virtual resistor, $R_{4}$, is placed in parallel with a capacitor, an extra voltage sensor will be required to monitor the component voltages. This method is more attractive in practice because it does not require problematic differentiation and the block diagram implementation is presented in Figure 27. Any combination of virtual resistors may be used to achieve the desired effect [31] [32].

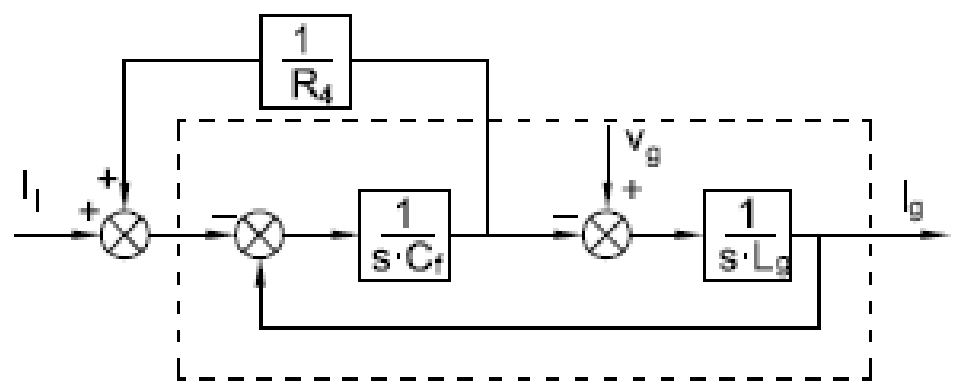

Figure 27: Block Diagram Implementation of Parallel Virtual Resistor [32]

\subsubsection{Lead-Lag Compensator}

This compensator usually introduces phase lead into the system which is normally required when a system needs to be damped. It requires monitoring of the filter capacitor voltages 
thereby introducing new sensors to the system. The capacitor voltages are passed through low pass filters and the outputs are subtracted from original capacitor voltages, which has the same effect of a high pass filter but does not run the risk of causing high harmonic disturbances. The resulting values are subtracted from the modulation voltage inputs. It will only introduce lead over a very small frequency band which can be tuned to the resonant frequency of the filter. The control for this damping method is simple but the extra cost of the sensors is disadvantageous [33] [34]. The transfer function for the lead-lag block is presented in equation 3.20 where the maximum lead angle is given by 3.21 , and occurs at a frequency given by equation 3.22 .

$$
\begin{gathered}
L(s)=k_{d} \frac{T_{d} s+1}{\alpha T_{d} s+1} \quad, \alpha<1 \\
\Phi_{\max }=\sin ^{-1}\left(\frac{1-\alpha}{1+\alpha}\right) \\
f_{\max }=\frac{1}{T_{d} \sqrt{\alpha}}
\end{gathered}
$$

Where $k_{d}$ and $T_{d}$ are the gain and time constant of the lead-lag transfer function respectively. Figure 28 illustrates the block diagram implementation of the lead-lag compensator within the grid-side converter. 


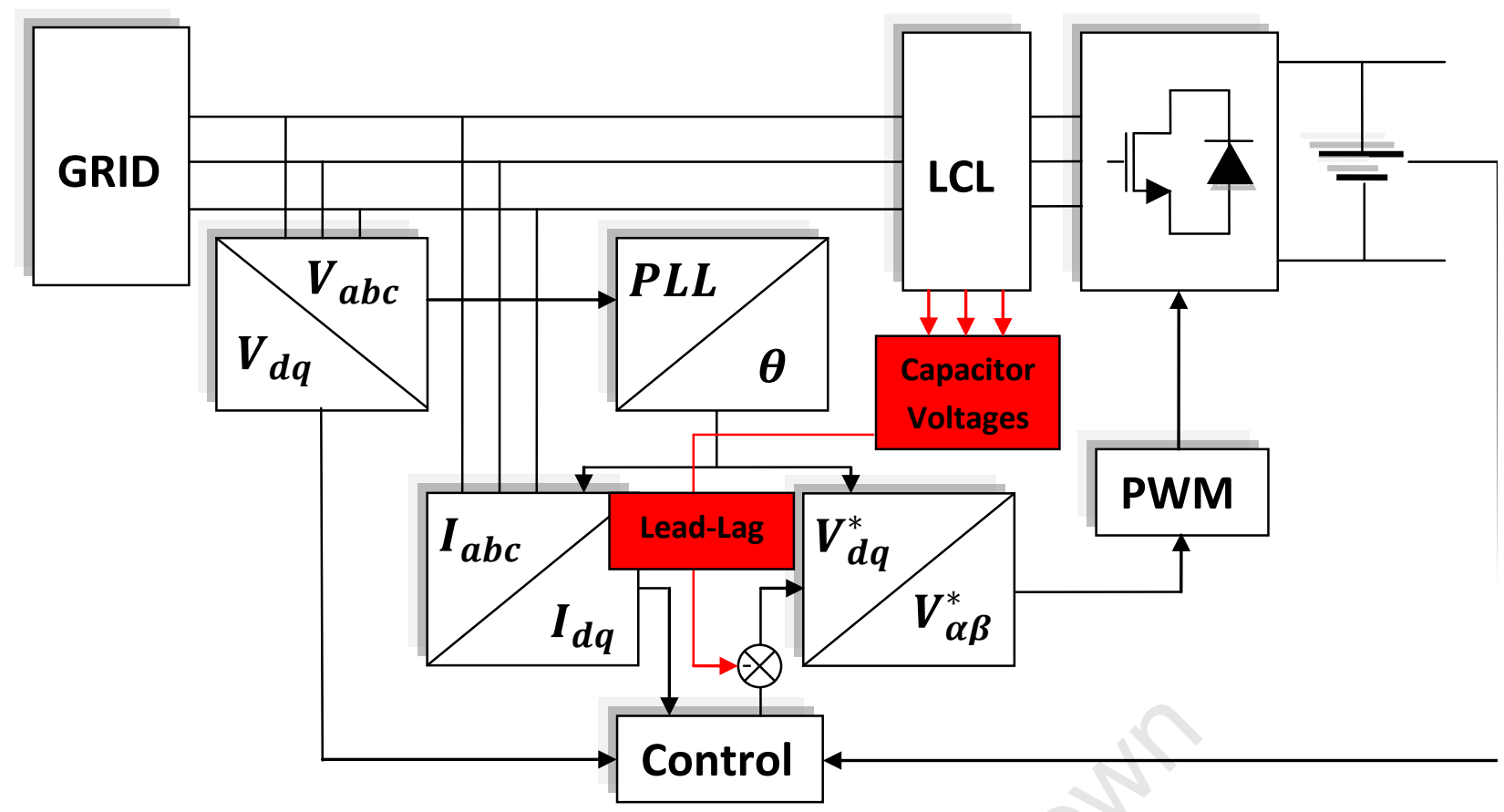

Figure 28: Implementation of Lead-Lag Active Damping

\subsubsection{Notch and Band stop Filters}

Notch filters and band stop filters provide a simple way of targeting and removing the resonant frequency of an LCL filter. The techniques are described in this section. A notch filter has the general transfer function below:

$$
G=\frac{s^{2}+2 D_{z} w_{z} s+w_{z}^{2}}{s^{2}+2 D_{p} w_{p} s+w_{p}^{2}}
$$

Where $D_{z}$ and $D_{p}$ are the zero and pole damping factors respectively. The zero damping factor needs to be less than the pole damping factor for there to be a negative notch. The difference between the respective damping factor values determines the bandwidth and magnitude of the notch. The resulting bode plot is shown in Figure 29. 


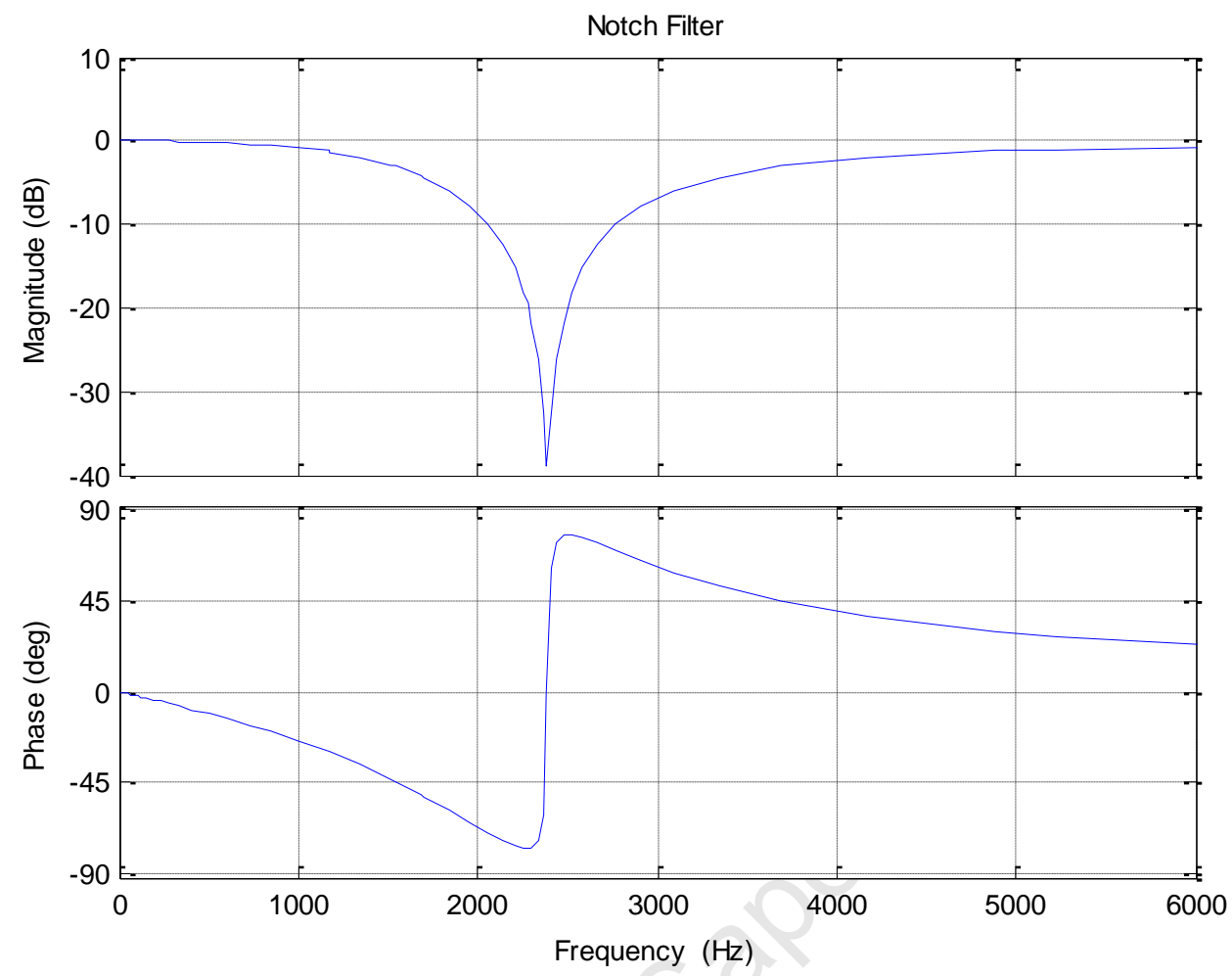

Figure 29: Notch Filter Bode Plot

The frequency can then be tuned to the resonant frequency of the filter and cancel out the resonance. Band stop filters have the same effect except that there is some attenuation over a range of chosen frequencies. Using band stop filters can cause high harmonic disturbances. To counteract this, a method is proposed that uses band pass filters and the outputs are subtracted from the inputs. Using either a notch or a band stop filter requires no extra sensing equipment and can simply be inserted into the control algorithm as it is shown in Figure 30. 


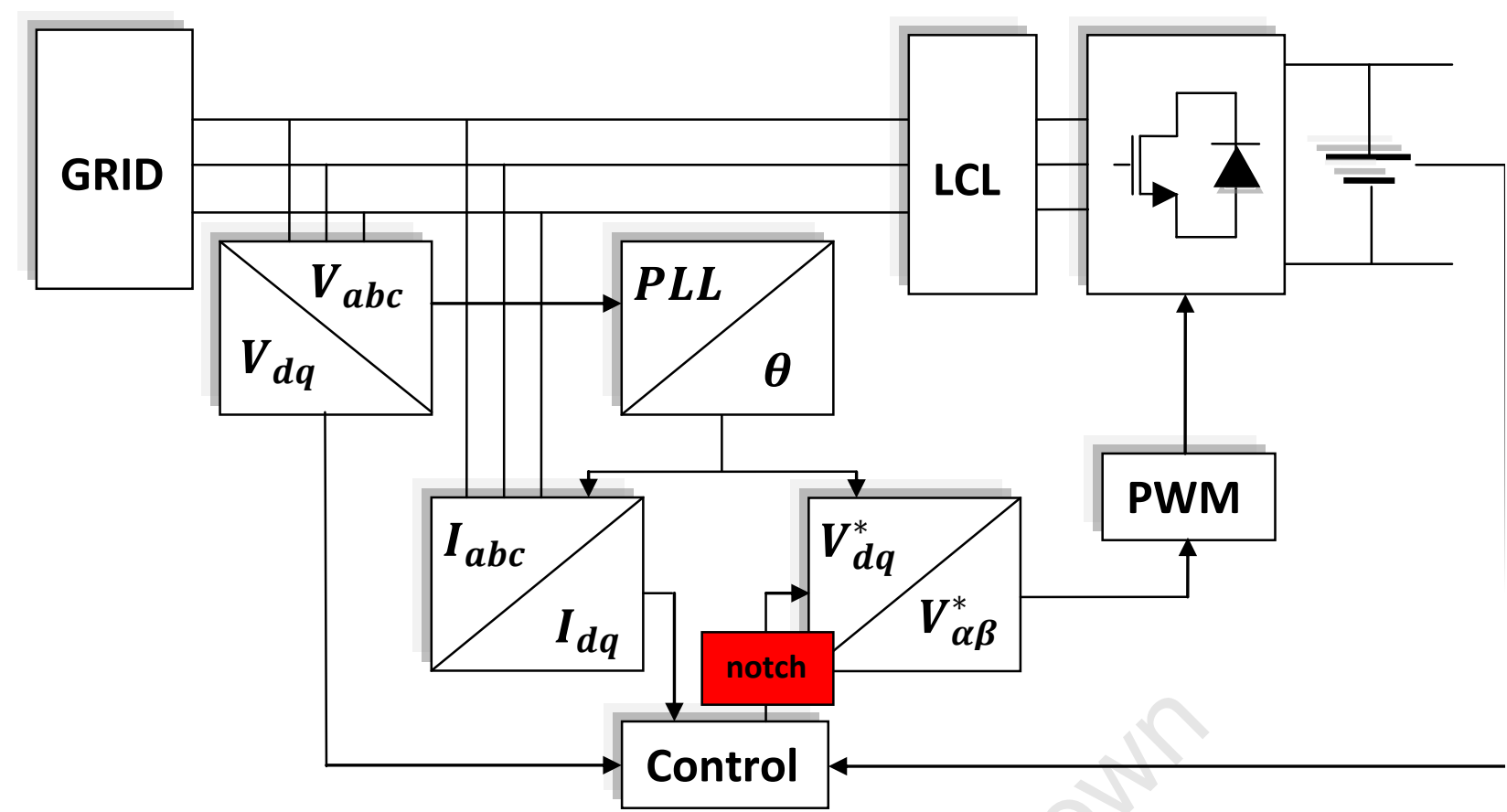

Figure 30: Notch Filter Implementation

The problem with notch or band stop filters is that if the resonant frequency of the LCL filter shifts due to changes in inductance or capacitance, the notch or band stop will no longer cancel out the exact resonant frequency. Both filters are attractive options for damping the filter resonance because they have no extra cost and are simple to implement in the control algorithm [32] [33]. This thesis will employ a band pass active damping technique due its simplicity and lack of extra components.

\subsubsection{Sensorless Operation}

It is possible to achieve sensorless active damping operation without any AC voltage sensors. This method can be used for both the lead-lag and notch or band stop methods of active damping. The voltage drop across the filter inductors is subtracted from the converter input signals. The estimation of the voltage drop can be implemented by power theory or by the differentiation of current. Both estimation methods use differentiation which can introduce noise into the system [33].

\subsection{Power Quality Issues}

The control scheme for the grid-side converter is dependent on the grid voltages being balanced for optimal control and is based in the synchronous reference frame. The 
transform from the $a b c$ reference frame to the $d q$ reference frame produces constant values for the $d q$ components if the grid voltages are perfectly balanced. If any sort of voltage unbalance is present, the $d q$ values will no longer be constant and will start to oscillate. This is illustrated in Figure 31 which compares the direct axis voltage waveform for both situations.

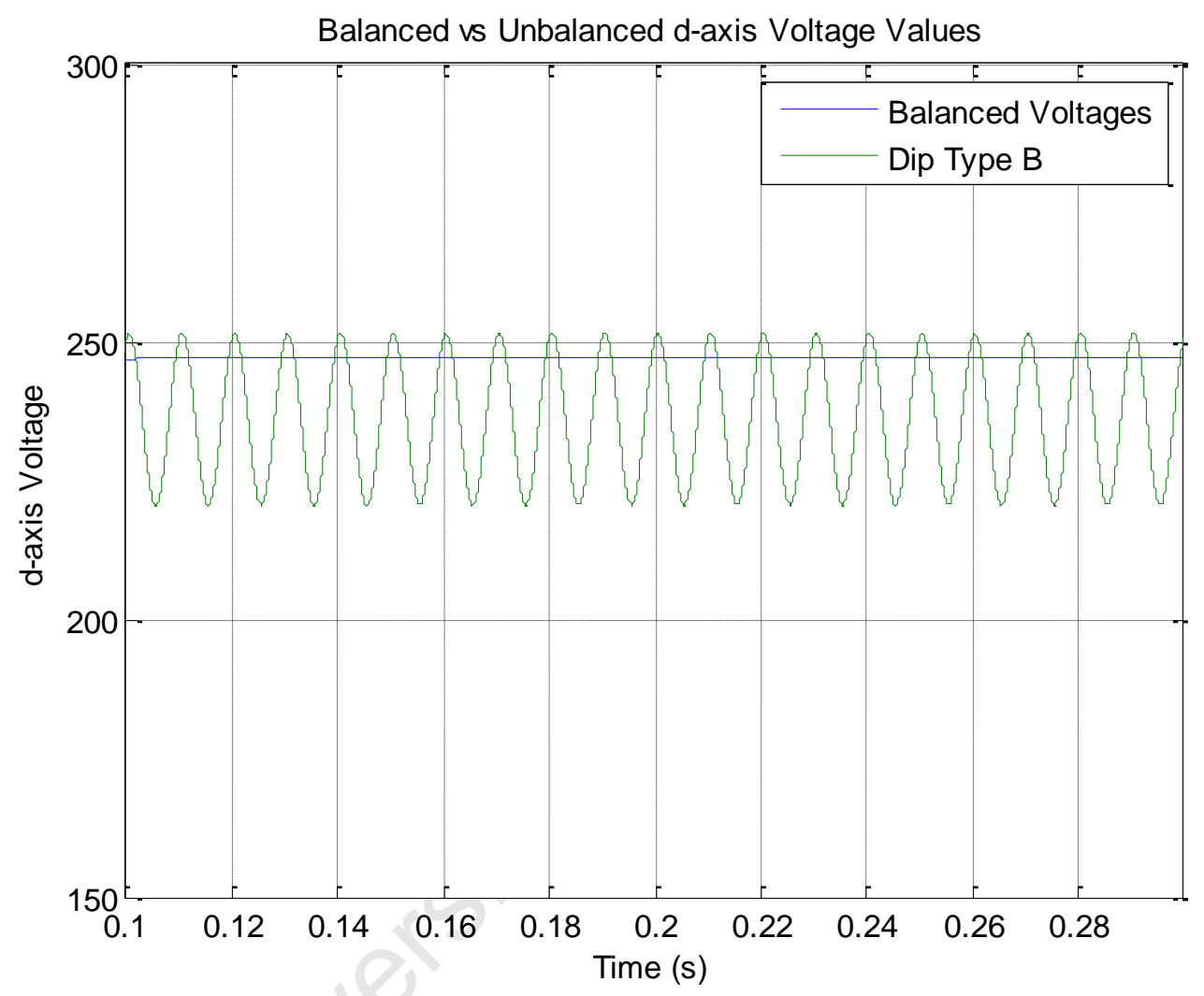

Figure 31: Comparison of Vd for Balanced and Unbalanced Voltages

The underlying issue that is created for the control can be explored by investigating the effect on the positive and negative sequence $d q$ values. For a perfectly balanced system, the positive sequence value will remain constant and the negative sequence value will be zero. When unbalance is introduced, a negative sequence value appears that rotates at twice the fundamental frequency in the positive sequence reference frame. In the negative sequence reference frame, the positive sequence vector rotates while the negative sequence value remains constant. A phasor diagram for unbalanced voltages where the resultant voltage phasor is composed of positive and negative sequence components is shown in Figure 32. 


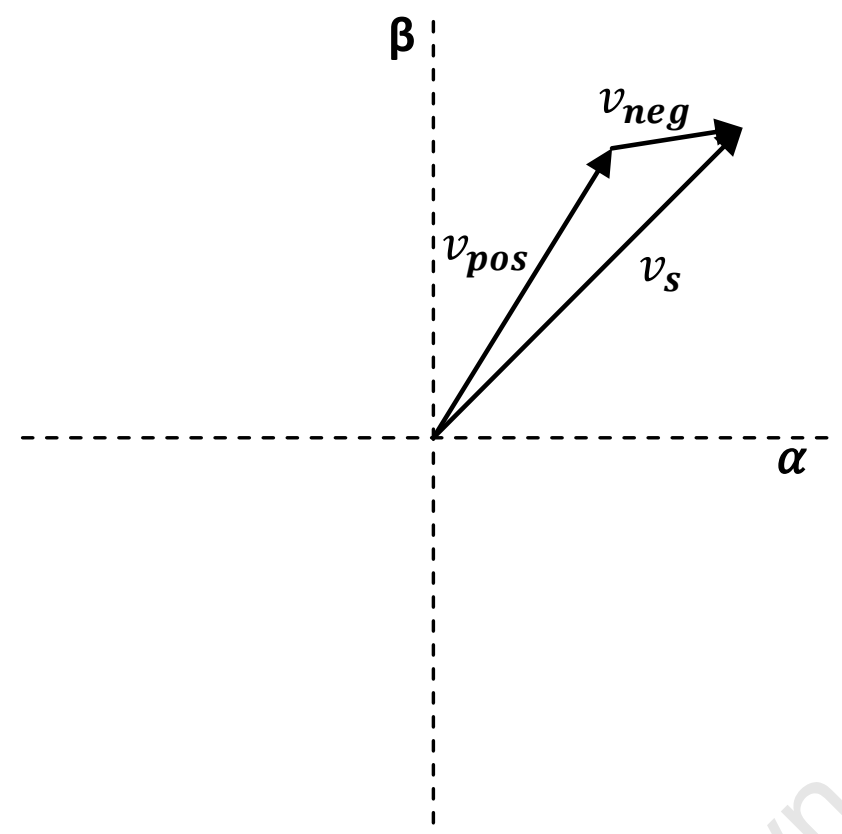

Figure 32: Positive and Negative Sequence Components of Unbalanced Three-Phase Currents

The effect of voltage unbalance in the stationary reference frame is illustrated in Figure 33 by means of an $\mathrm{XY}$ plot. The outer circle is obtained when the voltages are perfectly balanced. A type B voltage dip is then triggered and the inner oval shape is formed.

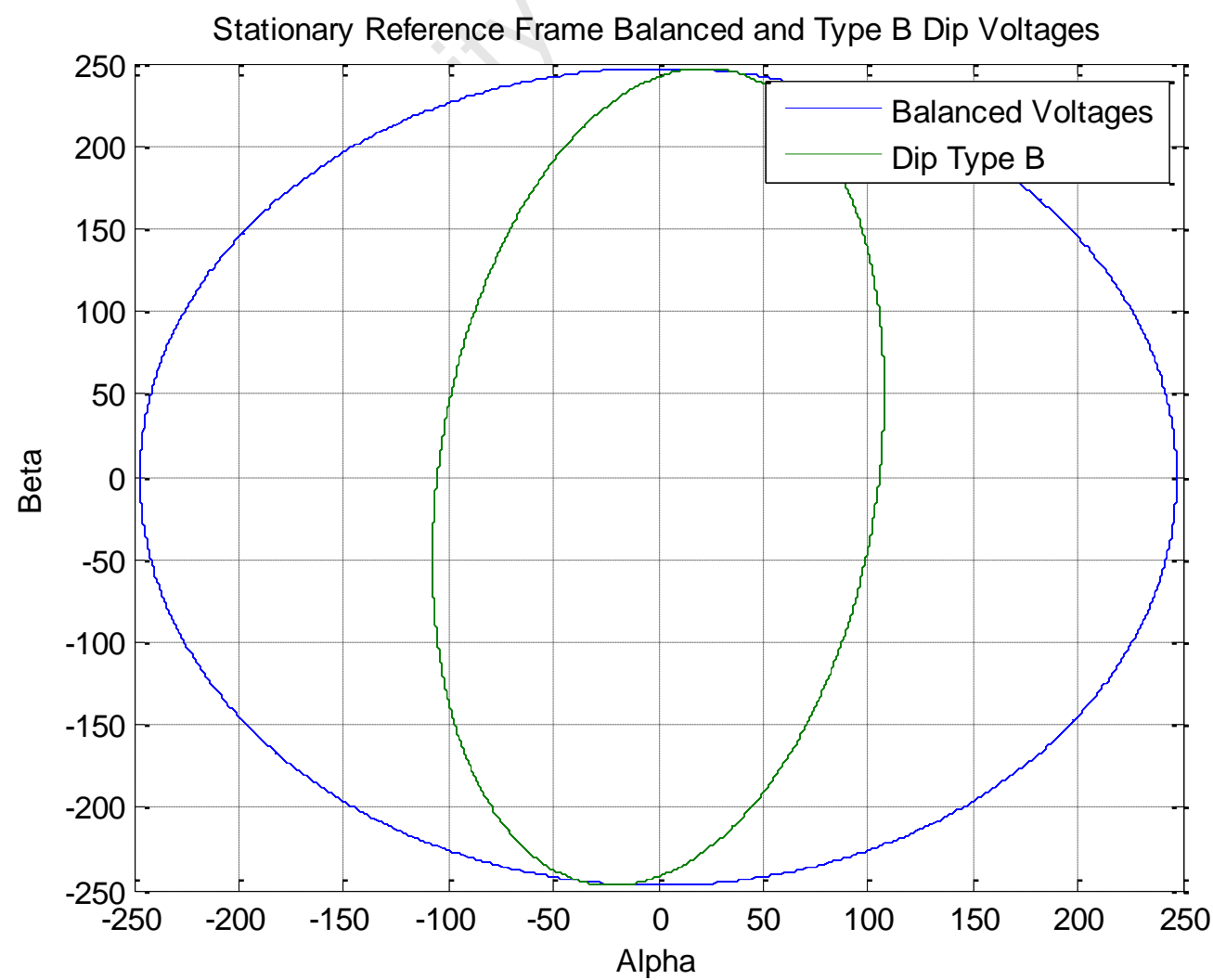

Figure 33: Effect of Voltage Unbalance in the Stationary Reference Frame 


\subsubsection{Sequence Separation Methods}

In order to analyse unbalanced voltage situations, the sequence components need to be extracted from the waveforms. There are two popular methods for sequence separation methods that are used in digital systems. Each has its own advantages and disadvantages. These methods will be discussed in this section.

\subsubsection{Filtering Methods}

Filtering methods are simple to implement digitally. Initially a standard $a b c$ to $\alpha \beta$ transformation is performed. The transformations into the positive and negative $d q$ reference frames are then made. If there is any unbalance, the positive reference frame will have a constant value with a sinusoidal waveform superimposed on it. The sinusoidal waveform is a negative sequence component that rotates at twice the fundamental frequency. The negative reference frame will have a sinusoidal positive sequence component superimposed on a constant value. The positive sequence component can be selected in the positive reference frame by a simple low pass filter since it is a dc value in the positive reference frame. Alternatively, the negative sequence component can be removed with a band stop or notch filter. The negative sequence component is selected in the same manner in the negative sequence reference frame. The transformation from the $\alpha \beta$ to the respective positive and negative reference frames is achieved through equations 3.30 and 3.31. Figure 34 illustrates this sequence extraction method. The transient response of this method is comparatively slow because of the low pass filter cut-off frequency therefore not making suitable for controllers that require a high bandwidth [35] [36].

$$
\begin{aligned}
& {\left[\begin{array}{l}
v_{d+} \\
v_{q+}
\end{array}\right]=\left[\begin{array}{cc}
\cos \theta & -\sin \theta \\
\sin \theta & \cos \theta
\end{array}\right]\left[\begin{array}{l}
v_{\alpha} \\
v_{\beta}
\end{array}\right]} \\
& {\left[\begin{array}{l}
v_{d-} \\
v_{q-}
\end{array}\right]=\left[\begin{array}{cc}
\cos \theta & \sin \theta \\
-\sin \theta & \cos \theta
\end{array}\right]\left[\begin{array}{l}
v_{\alpha} \\
v_{\beta}
\end{array}\right]}
\end{aligned}
$$




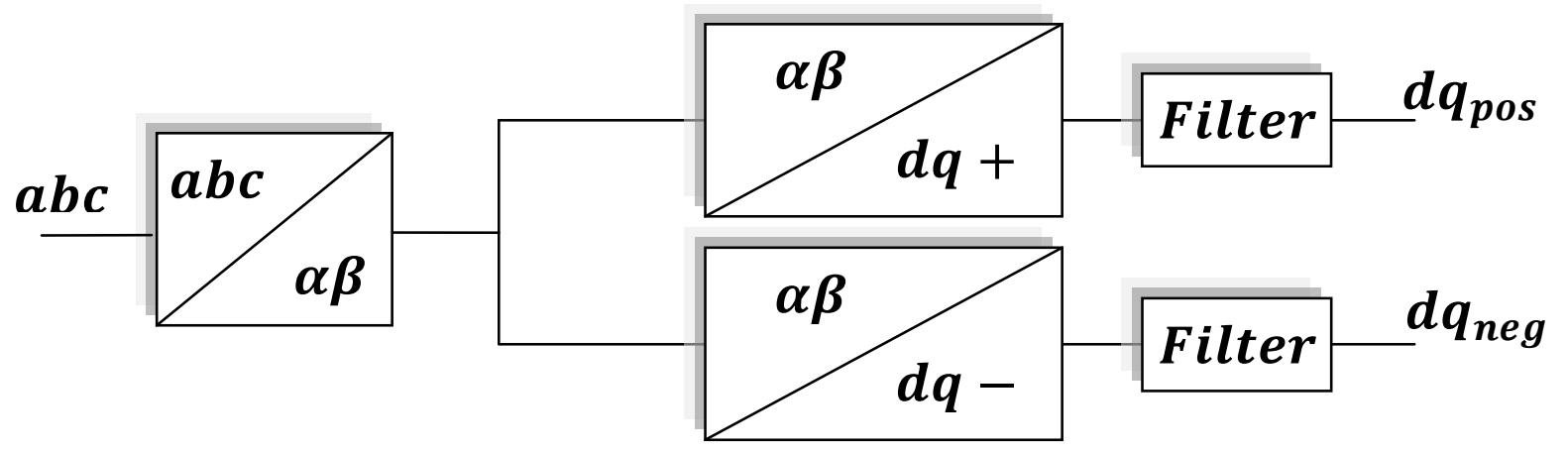

Figure 34:Filter Sequence Separation Method

\subsubsection{Signal Cancellation Methods}

These methods are based on the expressions 3.32 and 3.33 [37]. The most common version is delayed signal cancelation and has a transient settling time of $\frac{T}{4}$ which is inherent in the algorithm.

$$
\begin{aligned}
& e_{\text {pos }}^{(\alpha \beta)}=0.5\left(e^{(\alpha \beta)}(t)+j e^{(\alpha \beta)}\left(t-\frac{T}{4}\right)\right) \\
& e_{\text {neg }}^{(\alpha \beta)}=0.5\left(e^{(\alpha \beta)}(t)-j e^{(\alpha \beta)}\left(t-\frac{T}{4}\right)\right)
\end{aligned}
$$

Figure 35 illustrates the delayed signal cancelation method [36].

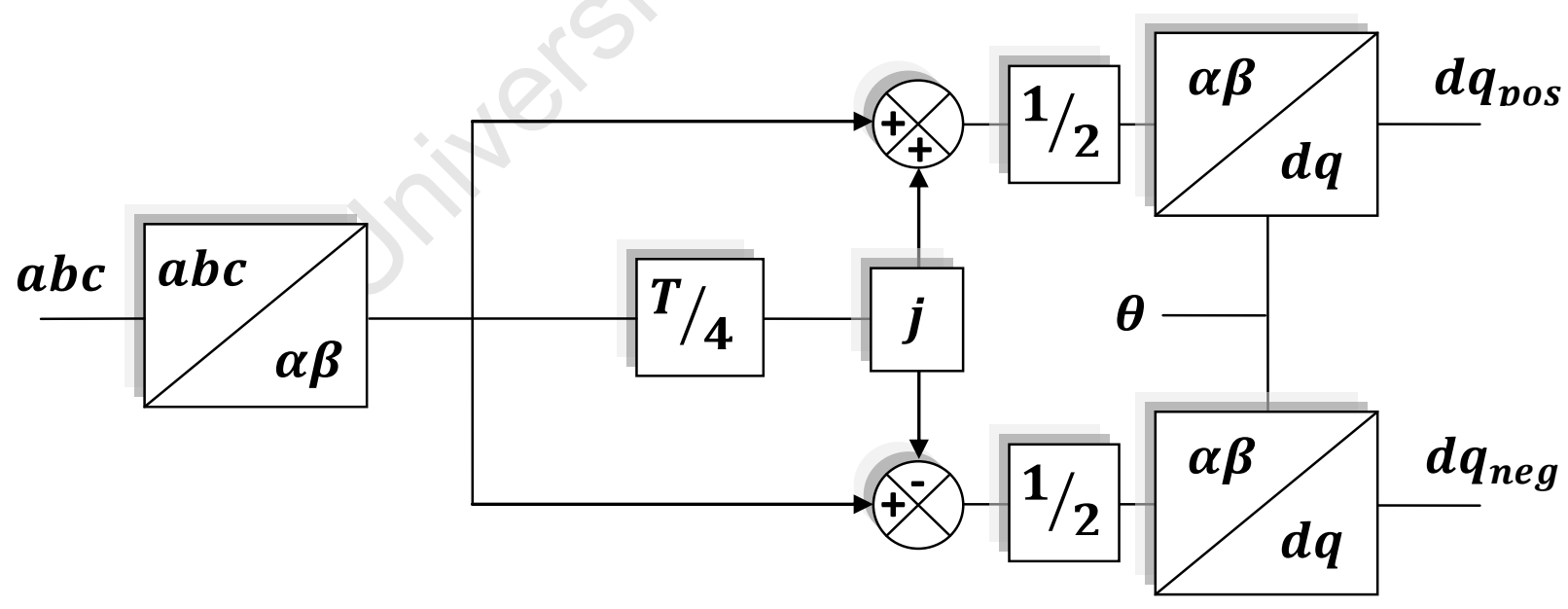

Figure 35: Delayed Signal Cancellation

In [37] a new method for sequence separation is proposed called differentiated signal cancellation which improves the delayed signal cancellation algorithm. It takes advantage of the fact that the $\alpha \beta$ input signals are sinusoidal in nature. A delay of $\frac{T}{4}$ would therefore shift them by $90^{\circ}$. Differentiating a sinusoidal signal has the same effect at the cost of only a 
single sample delay. The resulting value has to be multiplied by a gain of $\frac{1}{-\omega}$ in order to remove the $-\omega$ term that is introduced by the differentiation. A low pass filter is also introduced so that any noise that is amplified by the differentiation process is removed. However, this technique is still sensitive to any noise or harmonics that are present in the signals that are being used. The original $\alpha \beta$ values are also delayed by one sample period in order to synchronize all the signals. Figure 36 illustrates the modified sequence separation method. The differential signal cancellation technique was chosen to be implemented in this thesis because of its high speed transformations.

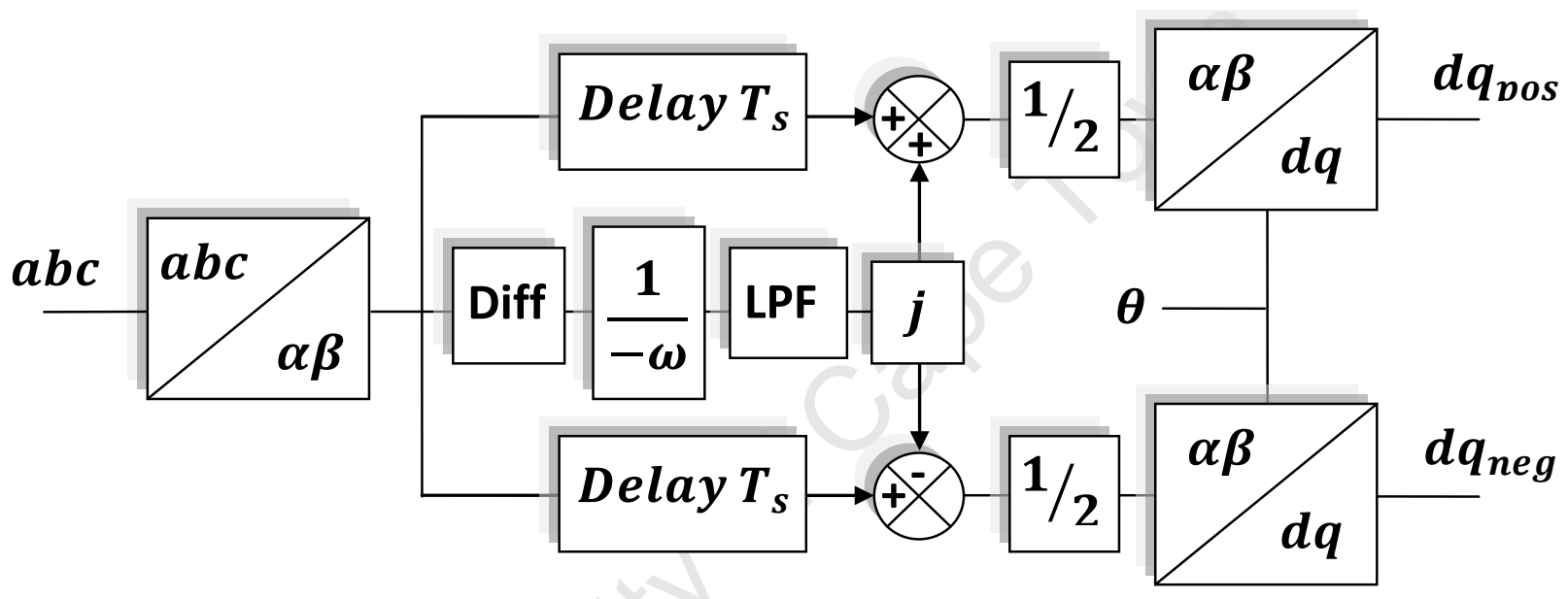

Figure 36: Differential Signal Cancellation

\subsubsection{Control Algorithm}

An alternative method of control is required to deal with low voltage situations. A versatile technique is to use the positive and negative sequence components for control. A combination of vector orientated control and dual vector current control is proposed in [38].

This allows for operation in many situations. In Figure 15 normal operating conditions are demanded for any situation while the voltages are still above 0.85p.u. Under these conditions there might still be some unbalance. The new control allows for balanced currents to be injected into the grid for these situations. When balanced currents with low total harmonic distortion are required, the negative sequence current components can simply be set to zero. Figure 37 shows the block diagram for the control algorithm. 


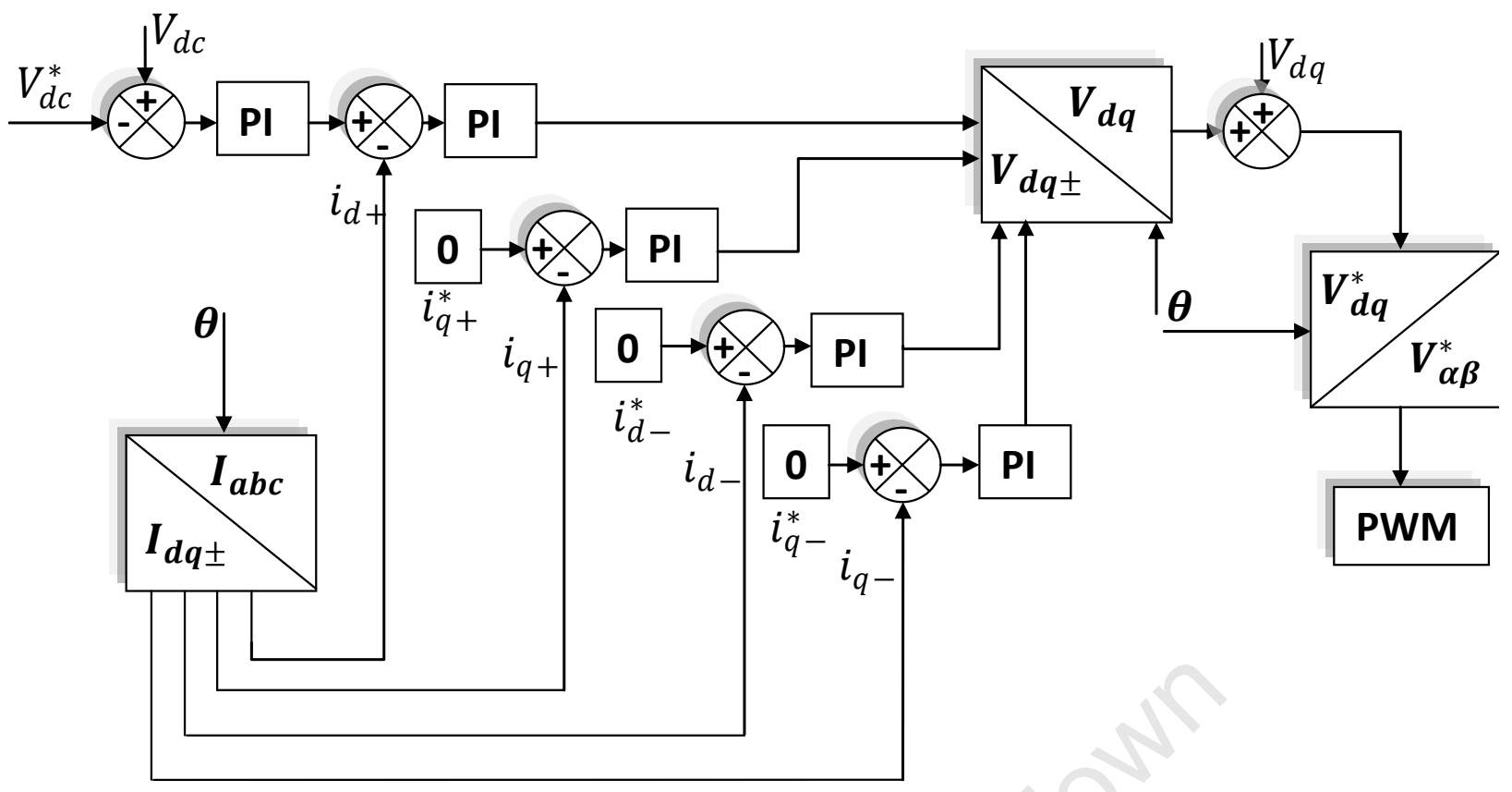

Figure 37: Dual Vector Current Control

\subsection{Conclusion}

This chapter introduced the necessary theory to understand the control of the generator and grid-side of the back-to-back converter and the respective control techniques were then developed in detail. LCL filter theory was investigated including various damping techniques. This thesis will employ a band pass active damping technique due its simplicity and lack of extra components. Methods for extracting the positive and negative sequence components were then investigated and the differential signal cancellation technique was chosen for its high speed transformations. Using the positive and negative sequence transformations, a control method was investigated which can supply balanced current for unbalanced grid voltages. The chosen techniques will be tested further through simulation and experimentation. 


\section{CHAPTER 4}

\section{CONTROLLER AND FILTER DESIGN}

This chapter introduces techniques for tuning PI controllers tuning and designing an $\mathrm{LCL}$ filter for the PM WECS. The theory that was developed in the previous chapters is applied to the design of the grid filter and PI controllers of the system.

\subsection{Control Tuning Techniques}

There are many tuning techniques available for calculating the gain values of a PI controller. In literature, the most common methods used in similar systems are the modulus optimum and the symmetrical optimum methods [40][43]. These two techniques are described below.

\subsubsection{Modulus Optimum}

In the system of Figure 38 , the modulus optimum technique attempts to comply with the following set of rules by tuning the controller parameters [39]. The magnitude of the closed loop transfer function should be equal to 1 from a frequency of zero to as high a frequency as possible:

1. $G_{r y}(0)=1$

2. $\frac{d^{m}\left|G_{r y}(j \omega)\right|}{d \omega^{m}}=0$ at $\omega=0$ for as many positive integers $m$ (starting from 1 ) as possible

Where $G_{r y}$ is the closed loop transfer function of the system in Figure 38.

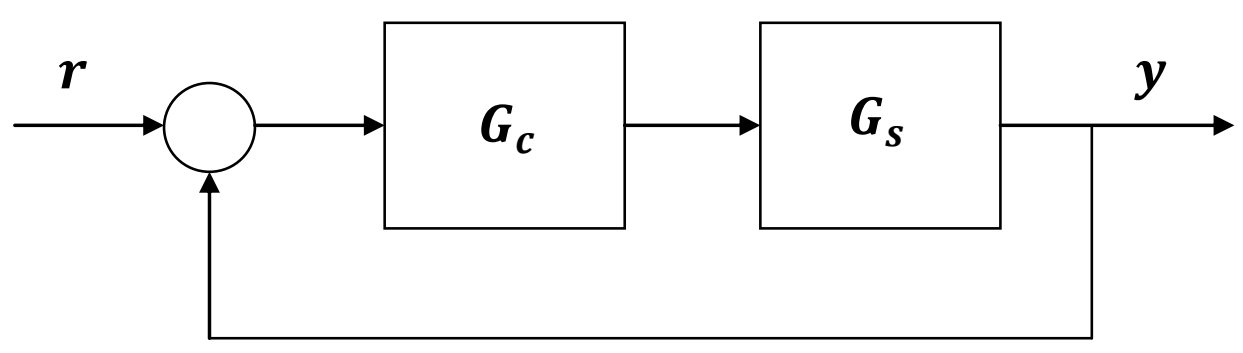

Figure 38: General Control Loop 
The first step in applying the modulus optimum technique described above is to find the closed loop transfer function of the system and then determine its order. The transfer function is then equated to the modulus optimum equation of equal order to solve for the controller values. The second and third order modulus optimum transfer functions are:

$$
\begin{gathered}
G_{M O}(s)=\frac{1}{1+\sqrt{2} T_{M O} s+T_{M O}^{2} s^{2}} \\
G_{M O}(s)=\frac{1}{\left(1+T_{M O} s\right)\left(1+T_{M O} s+T_{M O}^{2} s^{2}\right)}
\end{gathered}
$$

Where $T_{M O}$ is the time constant of the feedback control system designed by this control method [39].

\subsubsection{Symmetrical Optimum}

The symmetrical optimum design procedure is standard for open loop transfer functions with a double integration term when including the controller. A double integration term can be identified as two poles that are located at zero. The symmetrical optimum is based on the general equation below [40]:

$$
a_{i}^{2}+2 \sum_{j=1}^{i}(-1)^{j} a_{i-j} a_{i+j}=0
$$

Where $i$ is the number of adjustable controller parameters.

A PI controller has two adjustable parameters and the set of equations below are created:

$$
a_{1}^{2}-2 a_{0} a_{2}=0
$$




$$
a_{2}^{2}-2 a_{1} a_{3}=0
$$

Preitl and Precup then modified the equations 4.4 and 4.5 in [41] to ensure the maximum phase margin is obtained:

$$
\begin{aligned}
& a_{1}^{2}=\beta^{\frac{1}{2}} a_{0} a_{2} \\
& a_{2}^{2}=\beta^{\frac{1}{2}} a_{1} a_{3}
\end{aligned}
$$

Choosing a value of $\beta<4$ produces a phase margin of less than $36^{\circ}$ which is too small for practical systems. Furthermore, a value of $\beta>16$ produces a phase margin that is greater than $60^{\circ}$ which is not always necessary. Machaba and Braae used the fact that a value of $\beta<9$ results in one real pole and two which form a conjugate pair [42]. The variables that set the pole positions were related to the damping factor which produces the following characteristic equation [42]:

$$
s^{3}+(\alpha+2) \sigma s^{2}+\left(2 \alpha+1+\frac{1-\zeta^{2}}{\zeta^{2}}\right) \sigma^{2} s+\left(1+\frac{1-\zeta^{2}}{\zeta^{2}}\right) \sigma^{3}
$$

Where the real pole position is set by $\alpha \sigma$. Therefore $\alpha>1$ to ensure that the real pole is faster than the conjugate pair and $\zeta$ is the damping factor [42]. The characteristic equation can then be compared to a system with a third degree characteristic equation of the form presented in equation 4.9, which can then be used to determine the controller parameter values.

$$
a_{3} s^{3}+a_{2} s^{2}+a_{1} s+a_{0}
$$




\subsection{Filter Design}

When designing a filter it is important to set some performance goals as well as some component limitations. It is assumed that most of the current ripple is produced by the dominant harmonic at the switching frequency. The primary function of the filter is to eliminate the harmonics, thereby reducing the current ripple. To simplify the control and improve the efficiency of the system, the following limiting factors are imposed on the filter components [27]:

Limitation 1: $\quad$ The total inductance should be less than 0.1 per unit to limit the voltage drop across the filter. If the voltage drop is too large, a higher DC bus voltage would be required, which would increase the switching losses. The voltage that is dropped across an inductor can be expressed as:

$$
V=L \frac{d i}{d t}
$$

Limitation 2: The capacitor should be chosen to limit the decrease of the power factor. A general value used is less than $5 \%$.

Limitation 3: The resonant frequency should be in a range between ten times the fundamental frequency and half of the switching frequency.

Limitation 4: If passive damping is implemented, it should be enough to avoid oscillation but not too high so that losses are kept to a minimum.

The base values of the filter can be calculated from equations $4.11,4.12$ and 4.13 below.

$$
\begin{gathered}
Z_{b}=\frac{\left(E_{n}\right)^{2}}{P_{n}} \\
L_{b}=\frac{Z_{b}}{\omega_{n}}
\end{gathered}
$$




$$
C_{b}=\frac{1}{\omega_{n} Z_{b}}
$$

Where $E_{n}$ is the line-to-line voltage of the grid, $P_{n}$ - rated power and $\omega_{n}$ - fundamental frequency. The maximum values of the filter components can be calculated by using the aforementioned limitations and base values as follows:

\section{Step 1:}

In accordance with limitation 1, the maximum total inductance value is determined by

$$
L_{\max }=L_{b}(0.1)
$$

Step 2:

Limitation 2 sets the maximum capacitor value to:

$$
C_{f}=x C_{b}
$$

Where $x$ is the percentage of reactive power absorbed under rated conditions

Step 3:

The converter and grid inductors can be related by the ratio $r$

$$
L_{g}=r L_{I}
$$

Where $L_{g}$ is the grid side inductance and $L_{I}$ is the inverter side inductance of the LCL filter. The ratio $r$ sets the amount of attenuation achieved by the filter between the inverter and grid currents $i$ and $i_{g}$ at the switching frequency harmonic $h_{s w}$ in equation 4.17. The ripple 
current should be attenuated to a maximum value of 0.2 times converter side ripple current [27].

$$
\frac{i_{g}\left(h_{s w}\right)}{i\left(h_{s w}\right)}=\frac{1}{|1+r(1-a \cdot x)|}
$$

Where $a=L_{I} \cdot C_{b} \cdot \omega_{s w}^{2}$ and $\omega_{s w}$ is the switching fequency.

Step 4:

Once all of the component values have been chosen, the resonant frequency of the filter can be checked with equation 4.20 :

$$
F_{\text {res }}=\frac{1}{2 \pi} \sqrt{\frac{L_{t}}{L_{I} \cdot L_{g} C_{f}}}
$$

Where $L_{t}=L_{I}+L_{g}$ and is the total inductance of the LCL filter.

Step 5:

If all the components adhere to the rules, and passive damping is to be implemented, a rule of thumb is to add a resistor, $R$, which is equal to $\frac{1}{3}$ of the capacitor impedance at the resonant frequency, $\omega_{\text {res }}[29]$.

$$
R=\frac{1}{3 \omega_{r e s} C_{f}}
$$

\subsubsection{Calculated Filter Values}

The parameters of the system for which the LCL filter is designed are shown in Table 5. The filter component values were determined using the steps and calculations presented above and are presented in Table 6 below. 
Table 5: System Rated Parameters

\begin{tabular}{|c|c|}
\hline Parameter & Value \\
\hline$V_{L L}$ & $380 \mathrm{~V}$ \\
\hline $\boldsymbol{P}_{\text {rated }}$ & $6 \mathrm{~kW}$ \\
\hline $\boldsymbol{I}_{\text {rated }}$ & $9.09 \mathrm{~A}$ \\
\hline $\boldsymbol{F}_{\text {switching }}$ & $5000 \mathrm{~Hz}$ \\
\hline
\end{tabular}

Table 6: Calculated Filter Components

\begin{tabular}{|c|c|}
\hline Parameter & Value \\
\hline$Z_{b}$ & $24 \Omega$ \\
\hline$L_{b}$ & $76.4 m H$ \\
\hline$C_{b}$ & $132.6 \mu F$ \\
\hline$L_{\text {max }}$ & $7.64 m H$ \\
\hline$C_{f}(\mathrm{x}=4 \%)$ & $5 \mu F$ \\
\hline$L_{I}$ & $2.7 m H$ \\
\hline$r$ & 1.4 \\
\hline$L_{g}$ & $4.7 m H$ \\
\hline$f_{\text {res }}$ & $1719 H z$ \\
\hline
\end{tabular}

Figure 39 is a graphical representation of equation 4.17 at a switching frequency of $5 \mathrm{kHz}$. It shows the level of attenuation between the inverter and grid currents at the switching frequency harmonic as the ratio $r$ between the inverter and grid side inductors increases. 


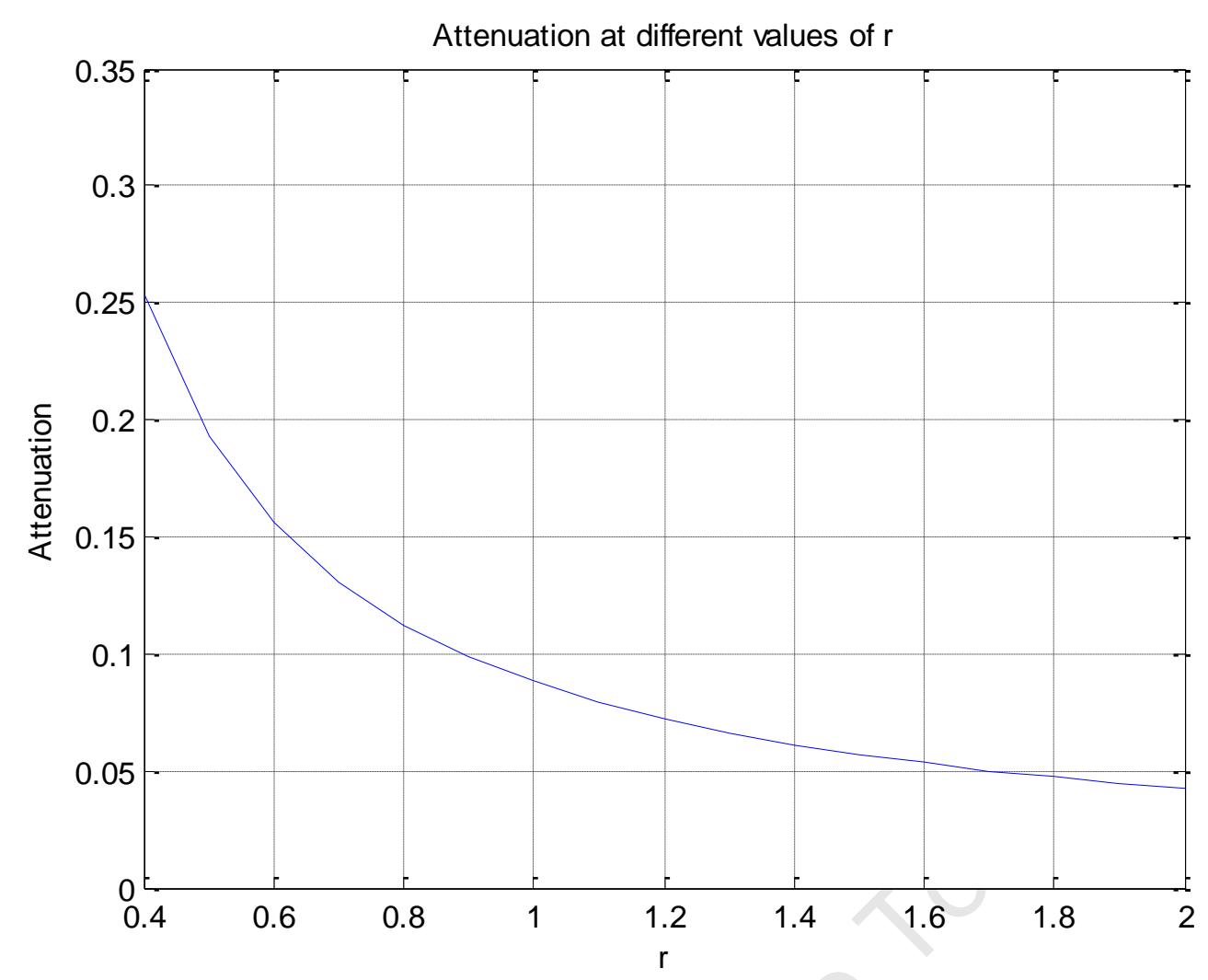

Figure 39: Relationship Between Attenuation and $r$

A value of 1.4 was selected to obtain an attenuation magnitude of less than $10 \%$.

The bode plot of the designed filter is presented in Figure 40.
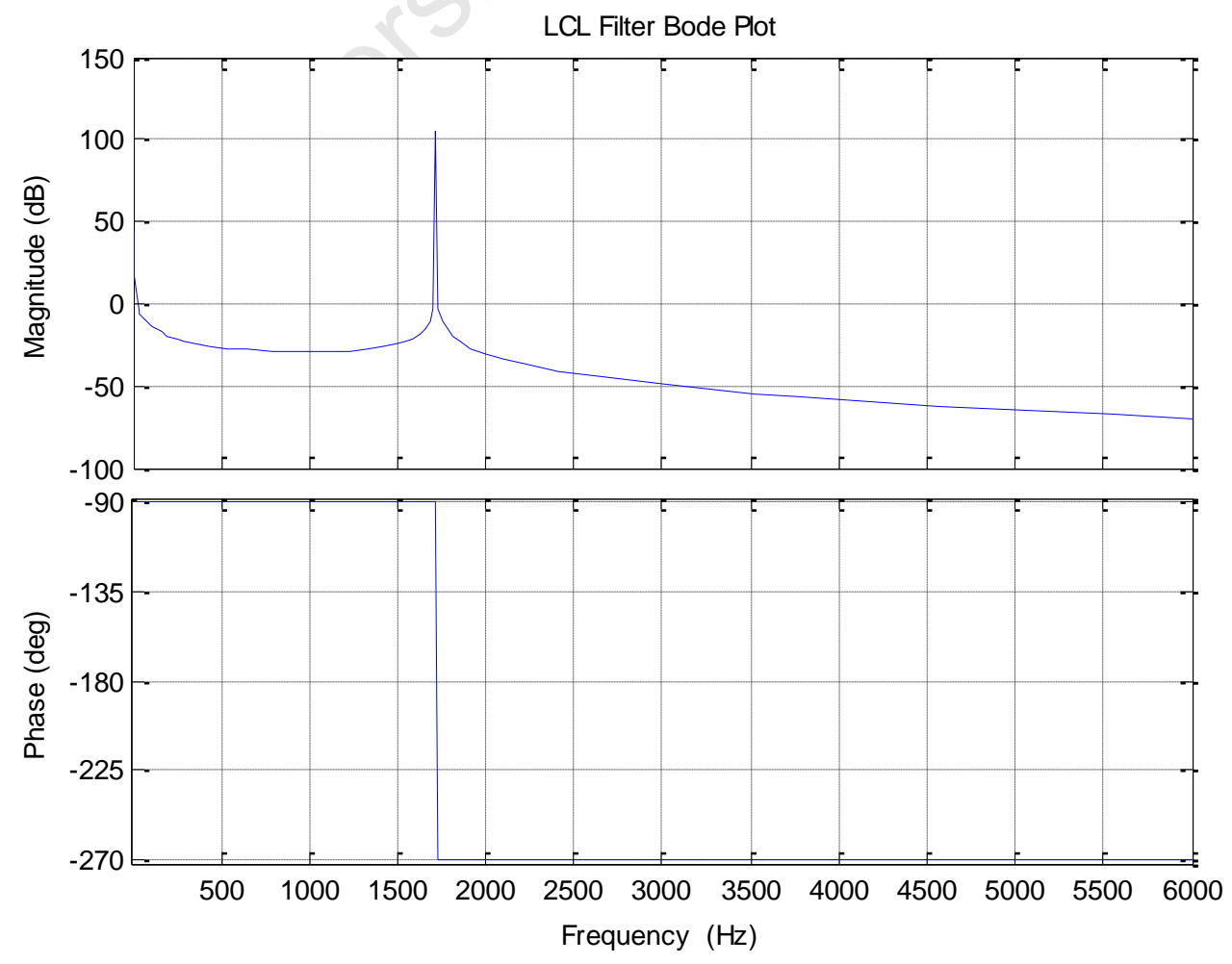

Figure 40: Bode Plot of LCL Filter 
Figure 40 shows that the resonant frequency is located at $1719 \mathrm{~Hz}$ which is equal to the calculated value of $1718.8 \mathrm{~Hz}$ using equation 4.20 . The resonant frequency therefore adheres to limitation 3. Furthermore, the attenuation at the switching frequency is greater than $50 \mathrm{~dB}$, however, the resonant frequency has a large gain and will need to be damped with either a passive or active technique described in section 3.3.2.

\subsection{PI Controllers}

As described in sections 3.2 and 3.3, the control algorithm for both the grid and generator sides of the back-to-back converters is made up of an inner current loop and an outer voltage/speed loop. This section illustrates how the PI controllers for the respective loops are tuned. The PI controller gains of both current loops are calculated using the modulus optimum technique, whilst the outer speed/voltage loops are calculated using the symmetrical optimum.

\subsubsection{Generator Current Controller}

The $d$ and q current loops are decoupled from each other and will have the same responses, therefore the q-axis equation is used to determine the machine transfer function because the $d$-axis current has no effect on the torque of the machine and is set to zero when using the MTA control technique described in section 3.2.2. It can be expressed as follows:

$$
i_{q}=\frac{V_{q}}{R_{S}}-\frac{L_{s}}{R_{S}} \Delta i_{q}-\frac{L_{s}}{R_{S}} \Delta \theta_{r} i_{d}
$$

Assuming that $i_{d}=0$, the equation simplifies to:

$$
i_{q}=\frac{V_{q}}{R_{s}}-\frac{L_{s}}{R_{s}} \Delta i_{q}
$$

Equation 4.23 is illustrated as a block diagram in Figure 41, which can then be simplified using block arithmetic to the transfer function of equation 4.22 . 


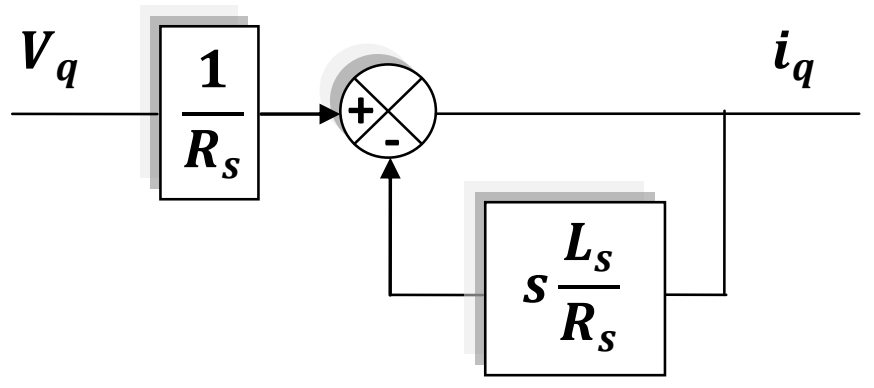

Figure 41: PMSG Quadrature-Axis Block Diagram

$$
G_{\text {machine }}=\frac{\frac{1}{R_{S}}}{\frac{L_{s}}{R_{S}} S+1}
$$

The block diagram for the current loop control is shown in Figure 42. The transfer functions for Figure 42 are given below.

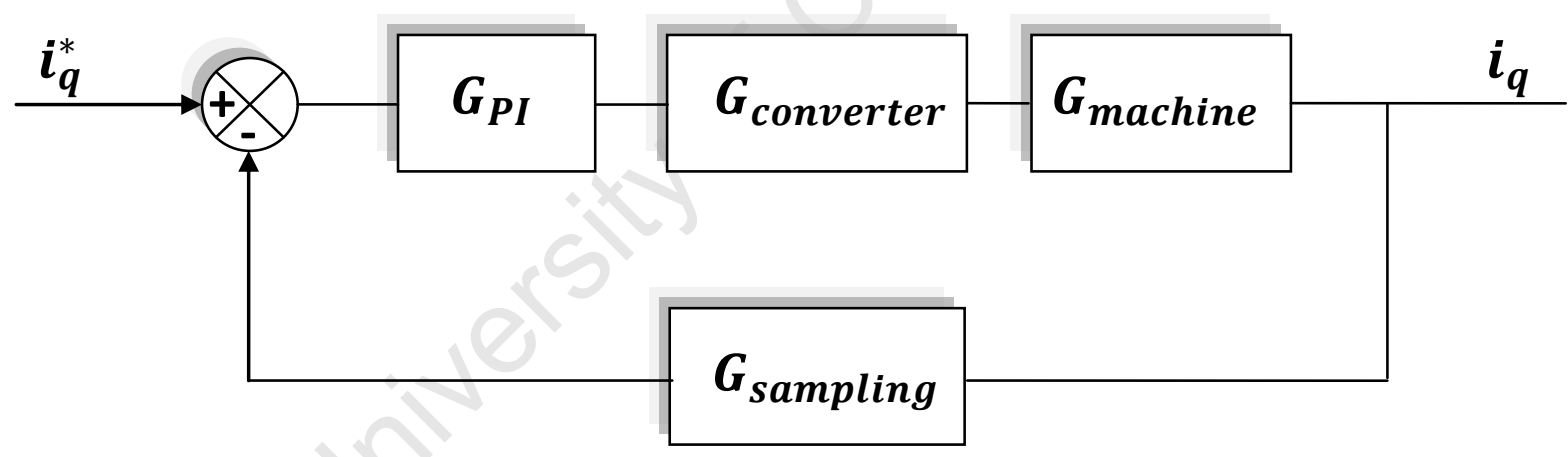

Figure 42: PMSG Current Control Loop

The transfer function of a standard PI controller is given by equation 4.23. The values $K_{p}$ and $T_{i}$ are the proportional gain and integral gain time constants respectively.

$$
G_{P I}=\frac{K_{p}\left(T_{i} s+1\right)}{T_{i} s}
$$

A converter can be modelled as a delay by the transfer function of equation 4.24, where $T_{\text {switching }}$ is equal to half the switching period. This is the statistical average delay that occurs at the converter [43]. 


$$
G_{\text {Converter }}=\frac{1}{T_{\text {switching }} S+1}
$$

The transfer function of the machine is presented in equation 4.25 which is obtained by simplifying the block diagram of Figure 42.

$$
G_{\text {machine }}=\frac{K_{E}}{T_{E} S+1}
$$

Where $K_{E}=\frac{1}{R_{S}}$ and $T_{E}=\frac{L_{S}}{R_{S}}$

The transfer function of the sampling is modelled as a delay with a time constant, $T_{\text {sampling }}$, equal to half the sampling period.

$$
G_{\text {Sampling }}=\frac{1}{T_{\text {sampling }} S+1}
$$

The modulus optimum method is used to tune the PI controller.

To simplify the transfer function of the loop, the small time constants $T_{\text {sampling }}$ and $T_{\text {switching }}$ can be combined into a single delay, $T_{c}$. The closed loop characteristic function of the system can be expressed as follows:

$$
G_{\text {characteristic }}=1+\frac{T_{E}}{K_{E} K_{p}} s+\frac{T_{E} T_{c}}{K_{E} K_{p}} s^{2}
$$

The PI controller values are determined by comparing equation 4.27 with the second order modulus optimum characteristic function of equation 4.1 and solving for $K_{p}, T_{i}$ and $K_{i}$. The controller values are determined by equations $4.28,4.29$ and 4.30 below.

$$
K_{p}=\frac{T_{E}}{2 K_{E} T_{c}}
$$




$$
\begin{gathered}
T_{i}=T_{E} \\
K_{i}=\frac{K_{p}}{T_{i}}
\end{gathered}
$$

Using the parameters of the experimental setup the resulting controller values are:

$$
\begin{gathered}
K_{p}=10.8333 \\
T_{i}=0.0086 \\
K_{i}=1259
\end{gathered}
$$

The values for the controller are used as starting values in the discrete analysis of the system using the Sisotool toolbox in Matlab. The transformation between the $s$ and $z$ domain is handled by Sisotool and sampling time of $0.0002(5 \mathrm{KHz})$ is used. The controller parameters are refined by moving the poles so that the damping factor is 0.707 . The new controller values are :

$$
\begin{gathered}
K_{p}=8.23 \\
T_{i}=0.0086 \\
K_{i}=957
\end{gathered}
$$


Figure 43 is the open loop root locus diagram of the current control loop and shows the poles at a damping factor of 0.707 within the unit circle, which shows that the system is stable.

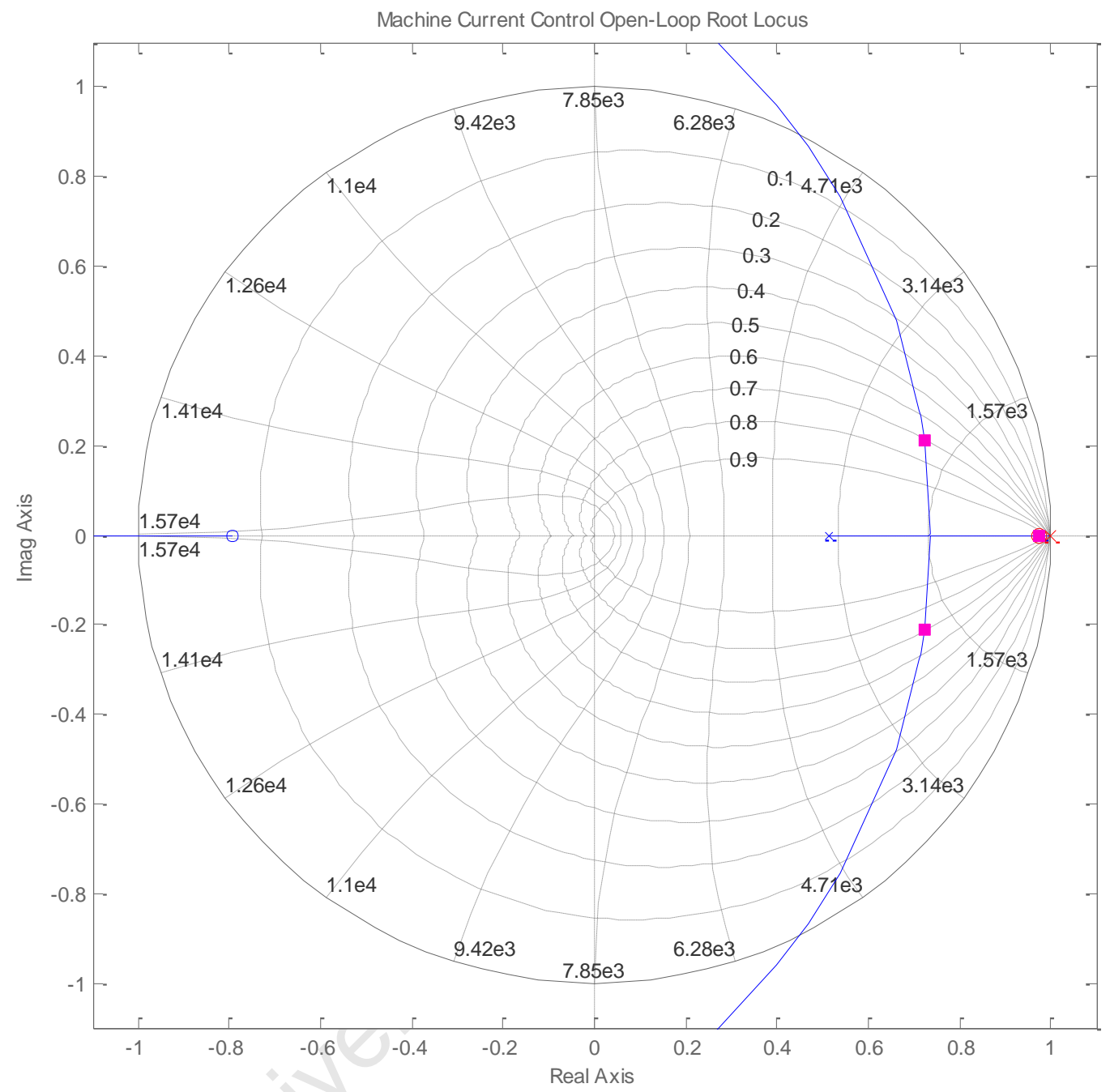

Figure 43: PMSG Current Control Loop Root Locus

More detailed information about the stability is obtained from the open loop bode plot in Figure 44 which shows that the current loop has a gain margin of 19.2 and a phase margin of 63.9. This indicates further that the loop is stable. 


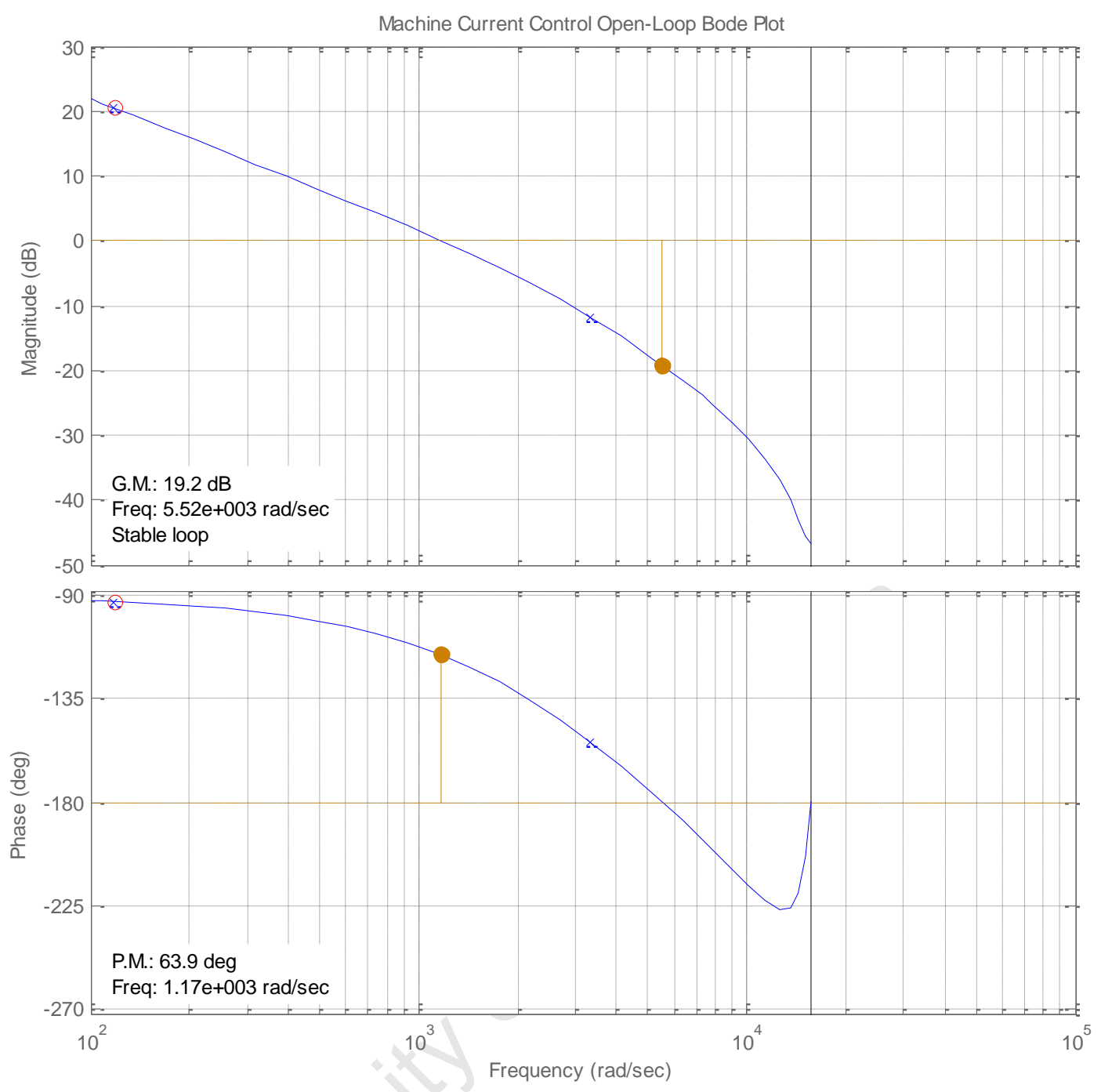

Figure 44: PMSG Current Control Loop Bode Plot

\subsubsection{Generator Speed Controller}

To determine the controller values for the speed control loop, the block diagram needs to be simplified by approximating the closed loop transfer function of the current loop. A simple technique is suggested in [40] where the closed loop is approximated by a delay of three times the sampling time. Figure 45 presents the comparison between the closed loop current response and the approximation response. 


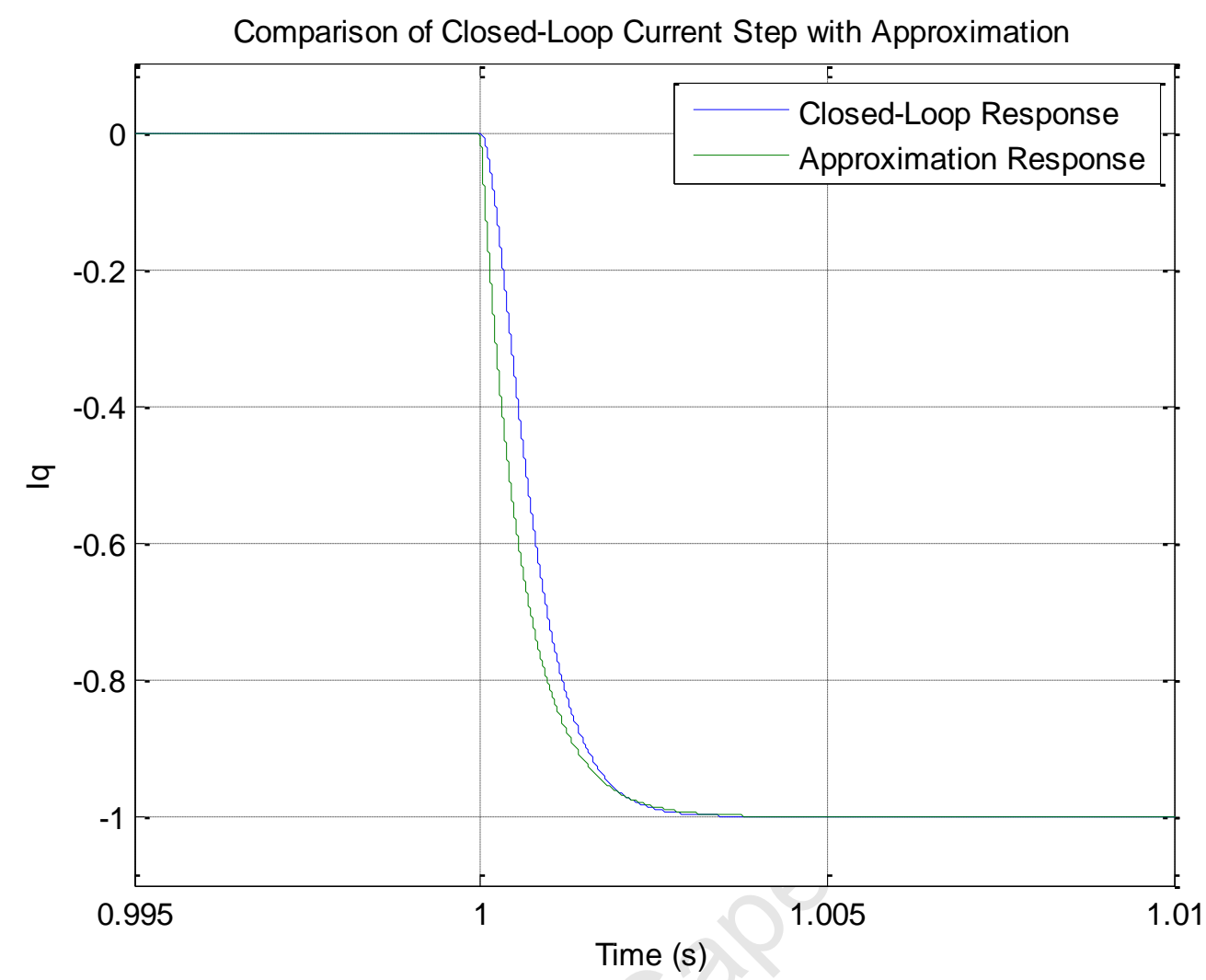

Figure 45: Comparison of Step Response between Current Loop and Approximation

The approximation response is close enough to the closed-loop response of the current loop, considering the greatly simplified transfer function that is used.

The block diagram of the speed control loop is illustrated in Figure 46.

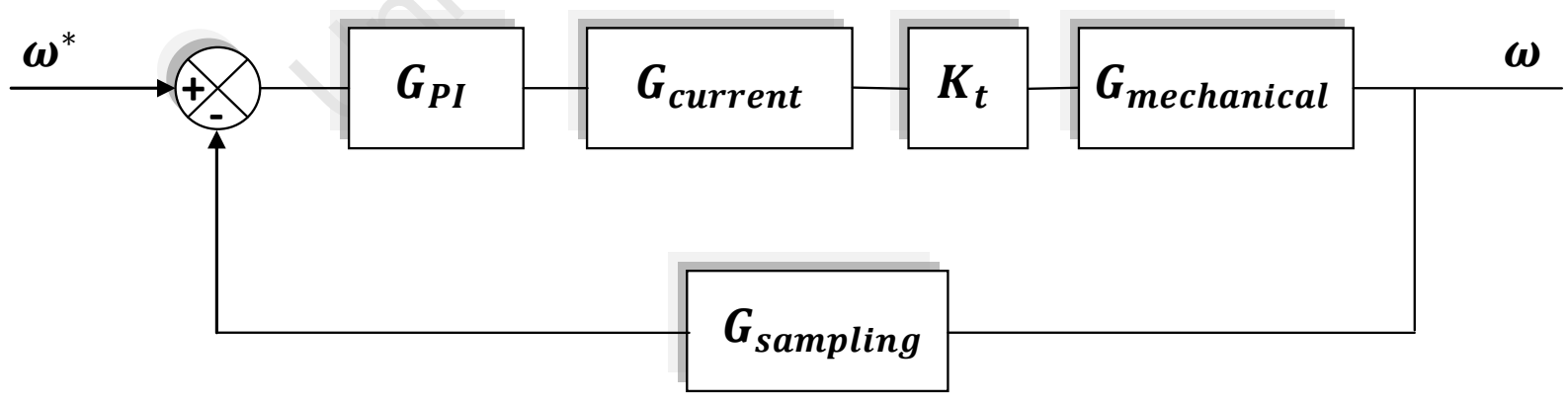

Figure 46: PMSG Speed Control Loop

The transfer functions of the PI controller and the sampling time are presented in section 4.3.1 by equations 4.23 and 4.26 respectively. The transfer function of $K_{t}$ is given by equation 4.31 . 


$$
K_{t}=\frac{3}{2} p^{2} \lambda_{a f}
$$

Where $p$ is the number of pole pairs in the machine and $\lambda_{a f}$ is the flux produced by the magnets of the machine.

The transfer function of the current loop is approximated by equation 4.32 and its time constant $T_{\text {current }}$ is equal to three times the sampling period as described above.

$$
G_{\text {current }}=\frac{1}{T_{\text {current }} s+1}
$$

The rotational mechanical characteristics, inertia $J_{S}$ and frictional damping $B_{S}$, of the machine are incorporated in equation 4.33.

$$
G_{\text {mechanical }}=\frac{K_{m}}{T_{m} s+1}
$$

where $K_{m}=\frac{1}{B_{S}}$ and $T_{E}=\frac{J_{S}}{B_{S}}$

The open loop transfer function of the system can then be given by equation 4.34

$$
G_{O L}=\frac{K_{p}\left(T_{i} s+1\right)}{T_{i} s} \frac{1}{T_{\text {current }} s+1} \frac{1}{T_{\text {sampling }} s+1} K_{t} \frac{K_{m}}{T_{m} s+1}
$$

To simplify the transfer function of the loop, the small time constants $T_{\text {sampling }}$ and $T_{\text {current }}$ can be combined into a single delay, $T_{s}$. Additionally, the mechanical transfer function can be approximated by

$$
T_{m} s+1 \cong T_{m} s
$$


This is due to equation 4.36 holding true around the cross-over frequency.

$$
\left|1+j \omega \frac{J}{B}\right| \simeq\left|j \omega \frac{J}{B}\right|
$$

The equation 4.34 then becomes:

$$
G_{O L}=\frac{K_{\sum}\left(T_{i} s+1\right)}{s^{2}\left(T_{s} s+1\right)}
$$

Where $K_{\Sigma}=\frac{K_{p} K_{t} K_{m}}{T_{i} T_{m}}$

The closed loop characteristic equation of equation 4.38 is compared to the symmetrical optimum transfer function 4.39 to determine the gain values.

$$
\begin{gathered}
G_{\text {characteristic }}=s^{3}+\frac{1}{T_{s}} s^{2}+\frac{K_{\Sigma} T_{i}+1}{T_{s}} s+\frac{K_{\Sigma}}{T_{s}} \\
s^{3}+(\alpha+2) \sigma s^{2}+\left(2 \alpha+1+\frac{1-\zeta^{2}}{\zeta^{2}}\right) \sigma^{2} s+\left(1+\frac{1-\zeta^{2}}{\zeta^{2}}\right) \sigma^{3}
\end{gathered}
$$

The controller gains are calculated by the set of equations presented below.

$$
\begin{gathered}
\sigma=\frac{1}{(\alpha+2) T_{s}} \\
K_{\Sigma}=T_{s}\left(1+\frac{1-\zeta^{2}}{\zeta^{2}}\right) \sigma^{3}
\end{gathered}
$$




$$
\begin{gathered}
T_{i}=\frac{\left(2 \alpha+1+\frac{1-\zeta^{2}}{\zeta^{2}}\right) \sigma^{2} T_{s}}{K_{\Sigma}} \\
K_{p}=\frac{K_{\Sigma} T_{i} T_{m}}{K_{t} K_{m}}
\end{gathered}
$$

The resulting controller values are:

$$
\begin{gathered}
K_{p}=2.68 \\
T_{i}=0.0084 \\
K_{i}=319
\end{gathered}
$$

The values for the controller are used as starting values in the discrete analysis of the system using the Sisotool toolbox in Matlab. A sampling time of $0.0002(5 \mathrm{KHz})$ is used and the poles are moved so that the damping factor is 0.707 . The new controller values are

$$
\begin{gathered}
K_{p}=3.16 \\
T_{i}=0.0084 \\
K_{i}=376
\end{gathered}
$$

Figure 47 is the open loop root locus diagram of the current control loop and shows the poles sitting at a damping factor of 0.707 within the unit circle suggesting system stability. 


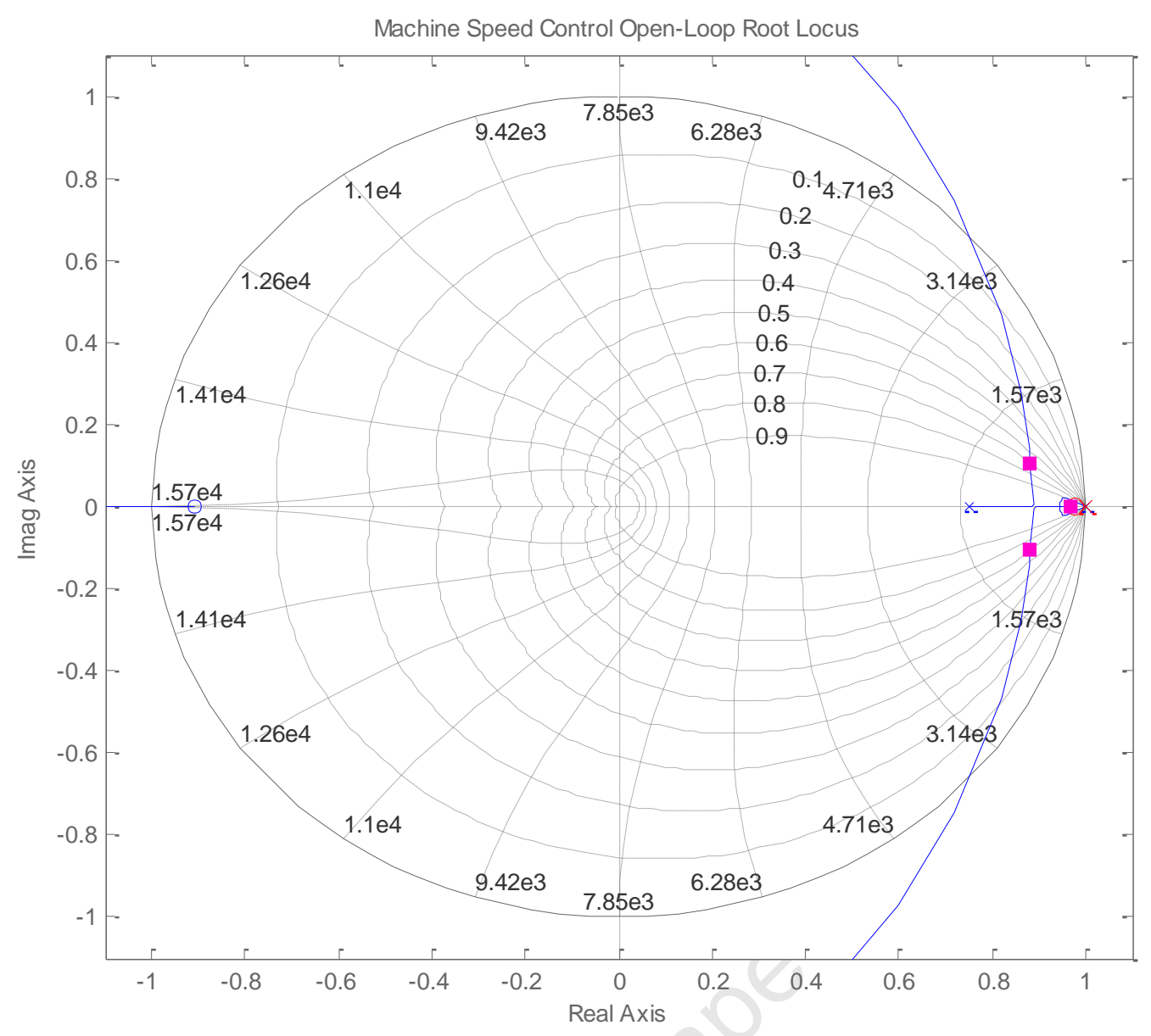

Figure 47: PMSG Speed Loop Root Locus

Figure 48 which shows that the current loop has a gain margin of 23.7 and a phase margin of 52.7, further indicating that the loop is stable.
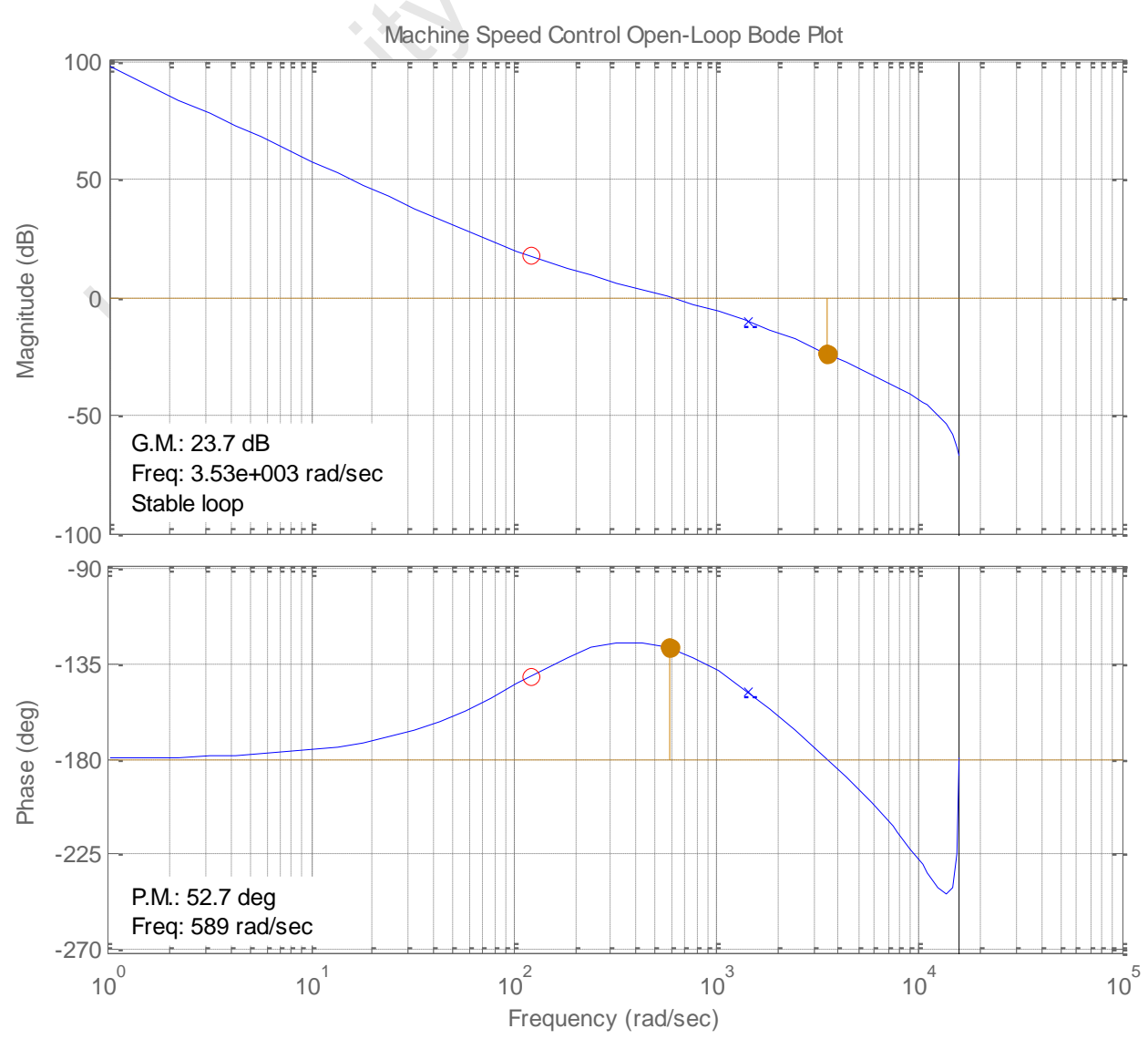

Figure 48: PMSG Speed Loop Bode Plot 
The gain values of the controller are modified when implemented in the experimental setup. This is due to the speed calculation method that is discussed further in section 6.3.2. An encoder is used which does not allow for accurate instantaneous speed to be calculated such as when a tachometer is used, thereby introducing a varying delay into the system that cannot be modelled easily. The gain values are decreased to the values presented below, in order to stabilise the system.

$$
\begin{gathered}
K_{p}=0.2 \\
T_{i}=0.2 \\
K_{i}=1
\end{gathered}
$$

\subsubsection{Grid Side Current Controller}

The grid side current loop is similar to the generator current loop, where only one of the current loops need to tuned because they have the same responses. The UPF control technique uses the $d$-axis current and sets the q-axis current to zero, therefore the $d$-axis current is used to tune the controller. The block diagram for the current loop is shown in Figure 49.

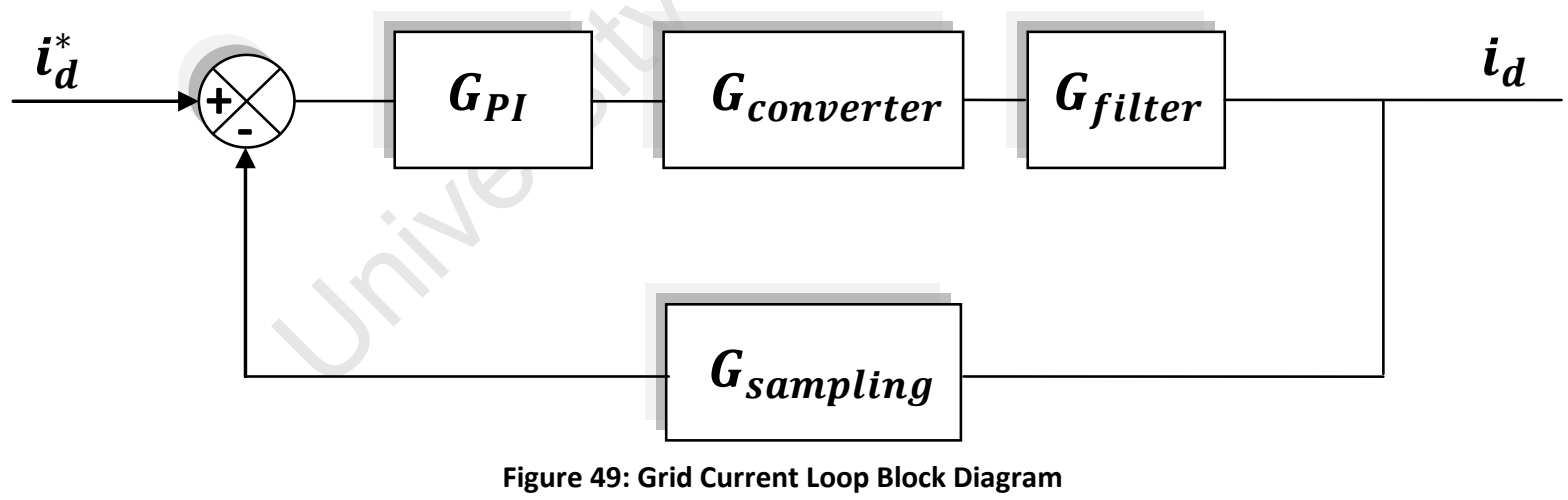

The transfer functions for the PI controller, converter and sampling time are given by equations $4.23,4.24$ and 4.26 respectively.

The transfer function of the Filter can be approximated by ignoring the filter capacitance and its transfer function is as follows: 


$$
G_{\text {Filter }}=\frac{K_{E}}{T_{E} S+1}
$$

Where $T_{E}=\frac{L_{I}+L_{g}}{R}, K_{E}=\frac{1}{R}$ and $\mathrm{R}$ is the sum of the parasitic resistances of the inverter and grid inductance, $L_{I}$ and $L_{g}$.

To simplify the transfer function of the loop, the small time constants $T_{\text {sampling }}$ and $T_{\text {switching }}$ can be combined into a single delay, $T_{c}$. The closed loop characteristic function of the system is:

$$
G_{\text {characteristic }}=1+\frac{T_{E}}{K_{E} K_{p}} s+\frac{T_{E} T_{c}}{K_{E} K_{p}} s^{2}
$$

Using the modulus optimum method to tune the PI controller the gains are determined by equations $4.46,4.47$ and 4.48 below.

$$
\begin{gathered}
K_{p}=\frac{T_{E}}{2 K_{E} T_{c}} \\
T_{i}=T_{E} \\
K_{i}=\frac{K_{p}}{T_{i}}
\end{gathered}
$$

The gain values that are obtained by these equations are indicated below and are used to start the discrete analysis using the Sisotool toolbox.

$$
\begin{aligned}
& K_{p}=21.14 \\
& T_{i}=0.0072
\end{aligned}
$$




$$
K_{i}=2936
$$

The poles are moved until damping of 7.07 is achieved in order to optimise the closed loop response and the resulting gains are:

$$
\begin{gathered}
K_{p}=13.51 \\
T_{i}=0.0072 \\
K_{i}=1876
\end{gathered}
$$

Figure 50 is the open loop root locus diagram of the current control loop and shows the poles located at a damping factor of 0.707 within the unit circle, thereby suggesting system stability.

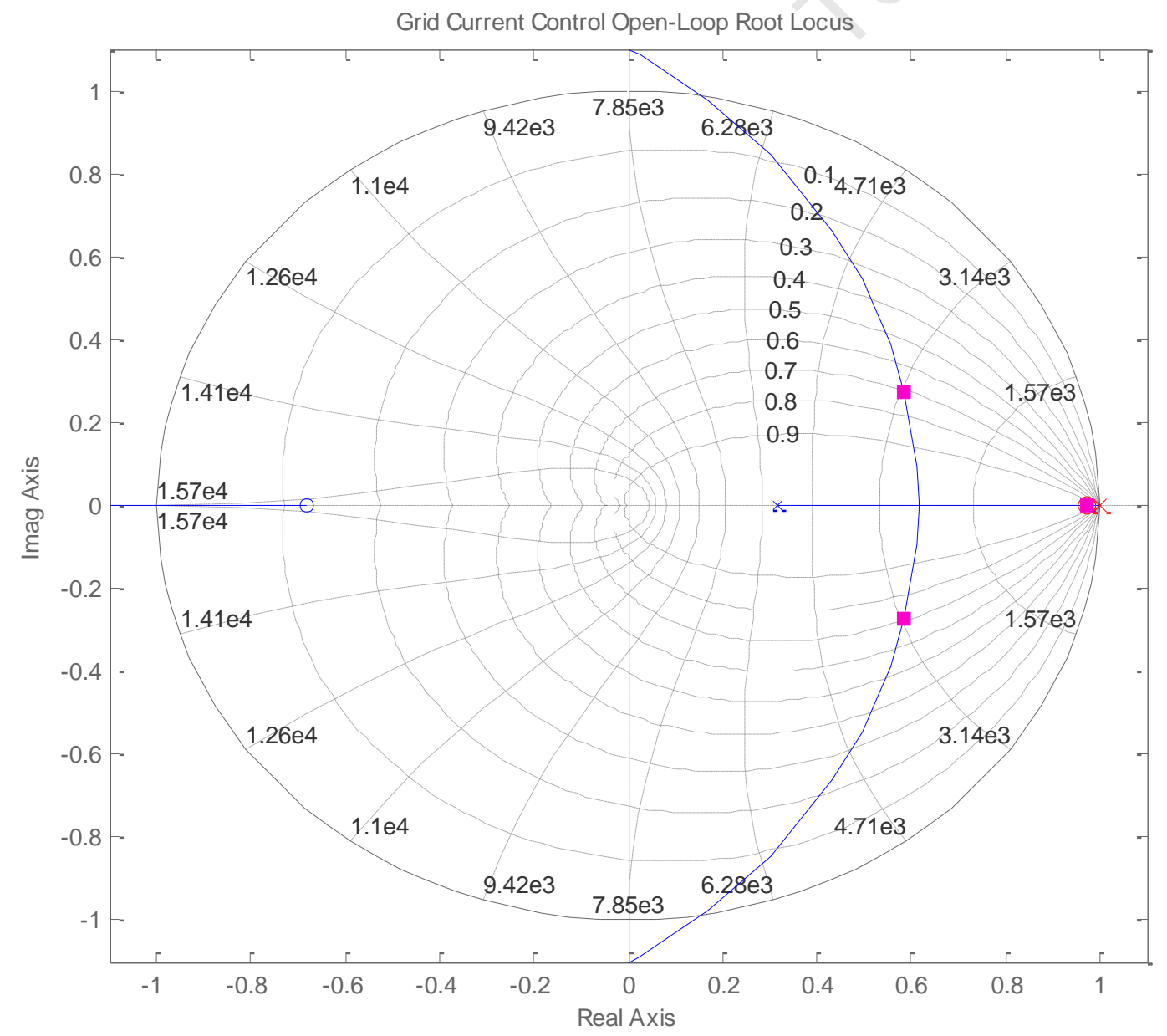

Figure 50: Grid Current Loop Root Locus 
Figure 51 which shows that the current loop has a gain margin of 16.7 and a phase margin of 63.4, further indicating that the loop is stable.

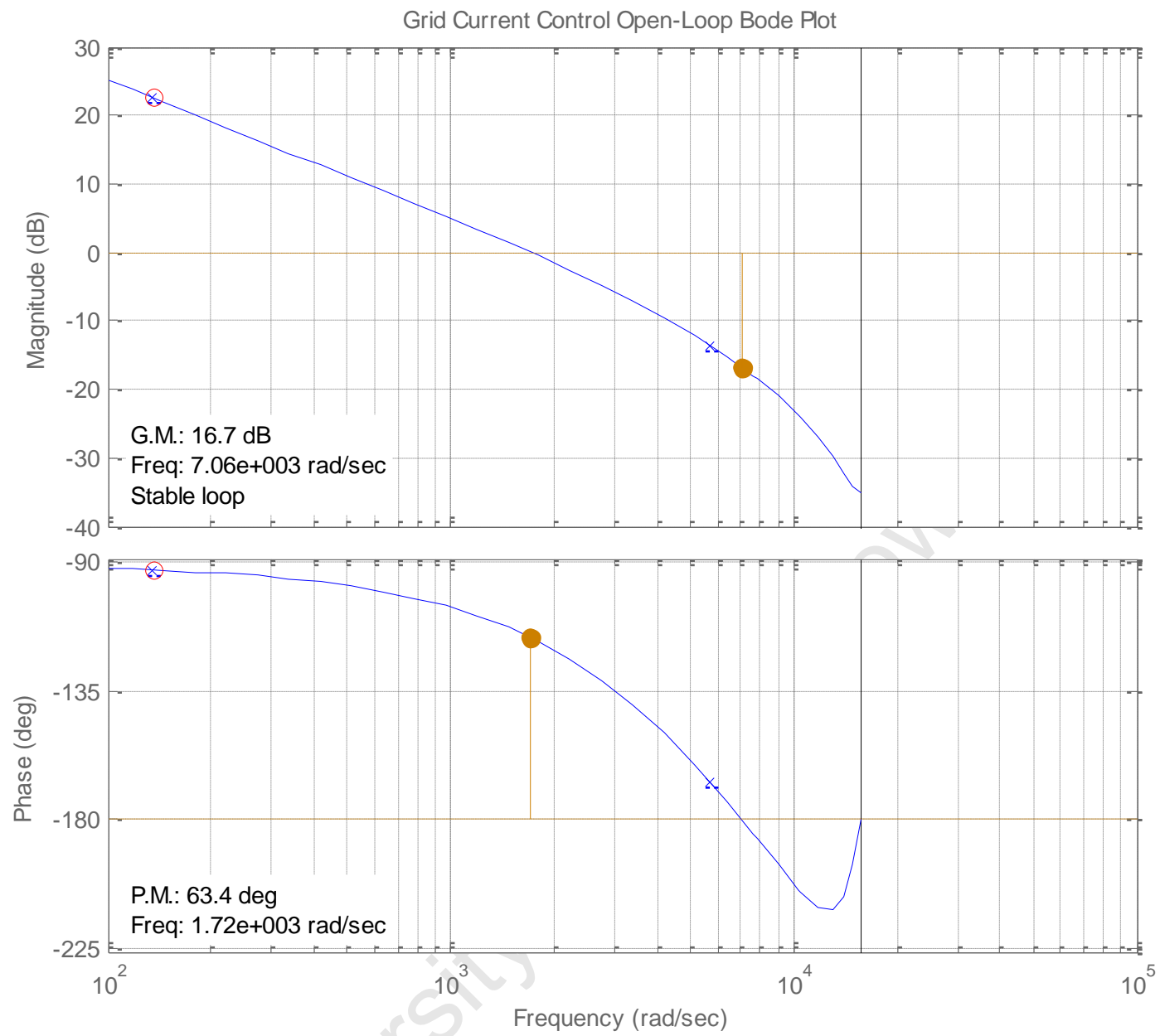

Figure 51: Grid Current Loop Bode Plot

\subsubsection{Link Voltage Control}

The outer DC Link Voltage control loop block diagram is illustrated in Figure 52. The closed loop current control is approximated by using the same method presented in section 4.3.1.

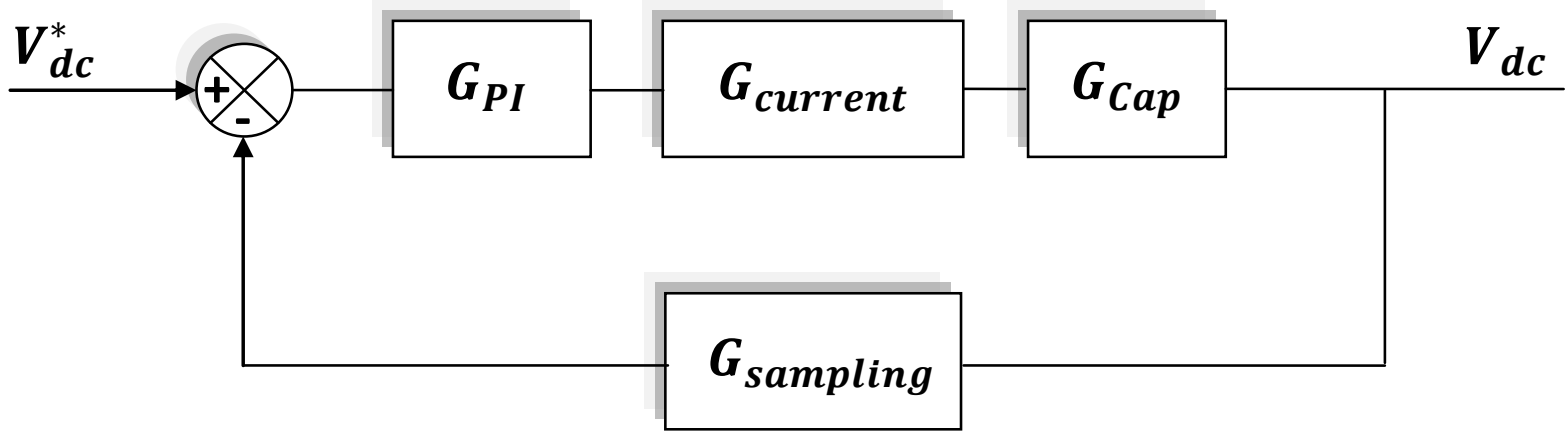

Figure 52: DC Link Voltage Loop Block Diagram 
The transfer functions of the PI controller, current loop and sampling are given by equations $4.23,4.32$ and 4.26 respectively, whilst the DC link capacitor transfer function is given by equation 4.49 , as:

$$
G_{c a p}=\frac{1}{C S}
$$

The resulting open loop transfer function for the DC link voltage control loop is:

$$
G_{O L}=\frac{K_{p}\left(T_{i} s+1\right)}{T_{i} S} \frac{1}{T_{\text {current } S+1}} \frac{1}{T_{\text {sampling }} s+1} \frac{1}{C s}
$$

To simplify the transfer function of the loop, the small time constants $T_{\text {sampling }}$ and $T_{\text {current }}$ can be combined into a single delay, $T_{s}$. The open loop characteristic function of the system can therefore be expressed as:

$$
G_{O L}=\frac{K_{\Sigma}\left(T_{i} s+1\right)}{s^{2}\left(T_{S} s+1\right)}
$$

Where $K_{\Sigma}=\frac{K_{p}}{T_{i} C}$

The closed loop characteristic function us given by equation 4.52 which can be compared to equation 4.39 to obtain the controller gains.

$$
G_{\text {characteristic }}=s^{3}+\frac{1}{T_{s}} s^{2}+\frac{K_{\Sigma} T_{i}+1}{T_{s}} s+\frac{K_{\Sigma}}{T_{s}}
$$

The resulting equations to determine the controller values are presented below:

$$
\sigma=\frac{1}{(\alpha+2) T_{s}}
$$




$$
K_{\Sigma}=T_{s}\left(1+\frac{1-\zeta^{2}}{\zeta^{2}}\right) \sigma^{3}
$$

$$
T_{i}=\frac{\left(2 \alpha+1+\frac{1-\zeta^{2}}{\zeta^{2}}\right) \sigma^{2} T_{s}}{K_{\Sigma}}
$$

$$
K_{p}=K_{\Sigma} T_{i} C
$$

The gain values that are determined by these equations are:

$$
\begin{gathered}
K_{p}=3.36 \\
T_{i}=0.0063 \\
K_{i}=533
\end{gathered}
$$

These values are again used as a starting point for the discrete analysis by means of the Sisotool toolbox and a damping factor of 0.707 changes the gain values to:

$$
\begin{gathered}
K_{p}=2.95 \\
T_{i}=0.0063 \\
K_{i}=468
\end{gathered}
$$

Figure 53 is the open loop root locus diagram of the voltage control loop and shows the poles at a damping factor of 0.707 within the unit circle, thereby suggesting system stability. 


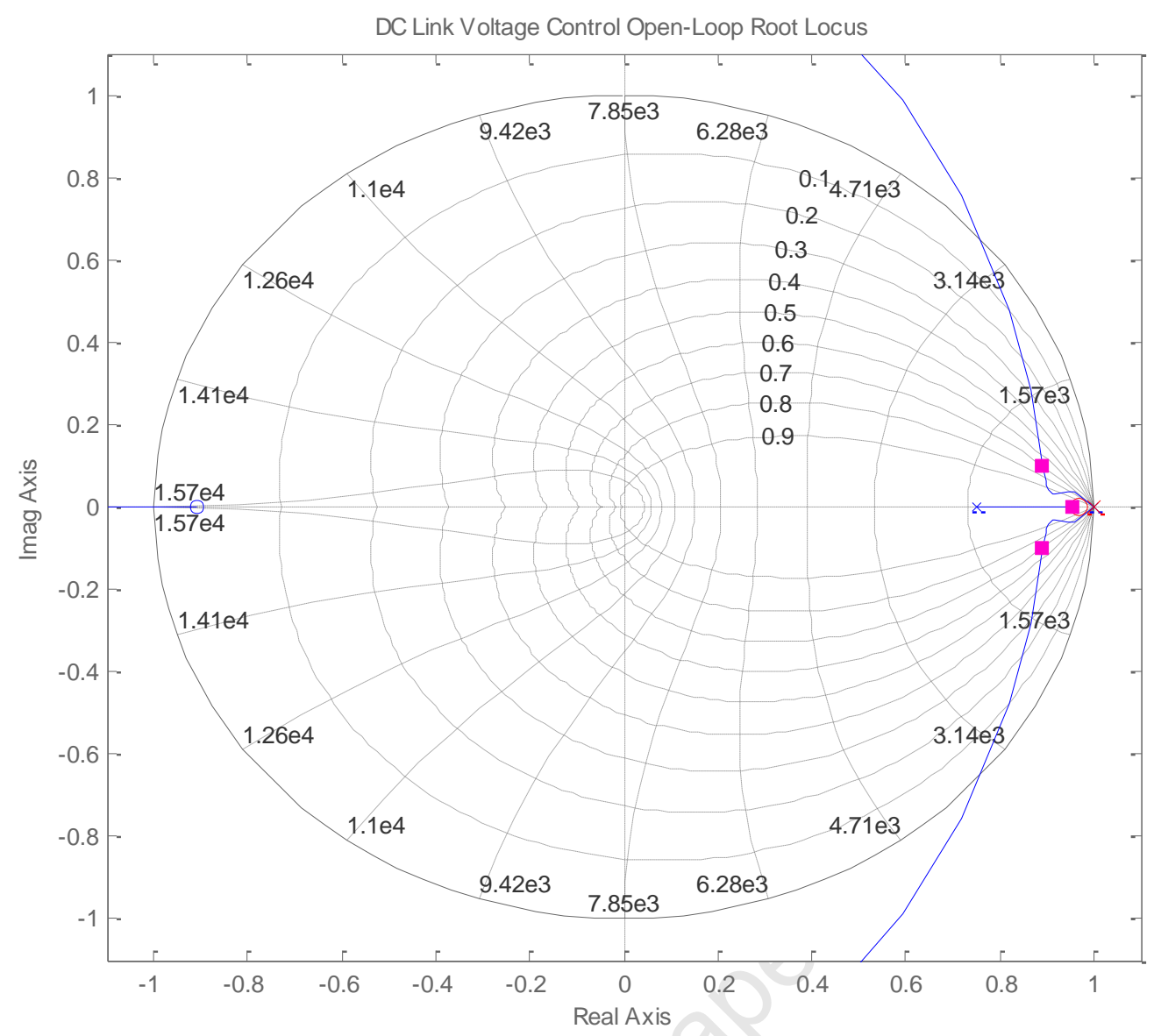

Figure 53: DC Link Voltage Loop Root Locus

Figure 54 shows that the voltage loop has a gain margin of 23.6 and a phase margin of 48.9, which further indicates that the loop is stable. 


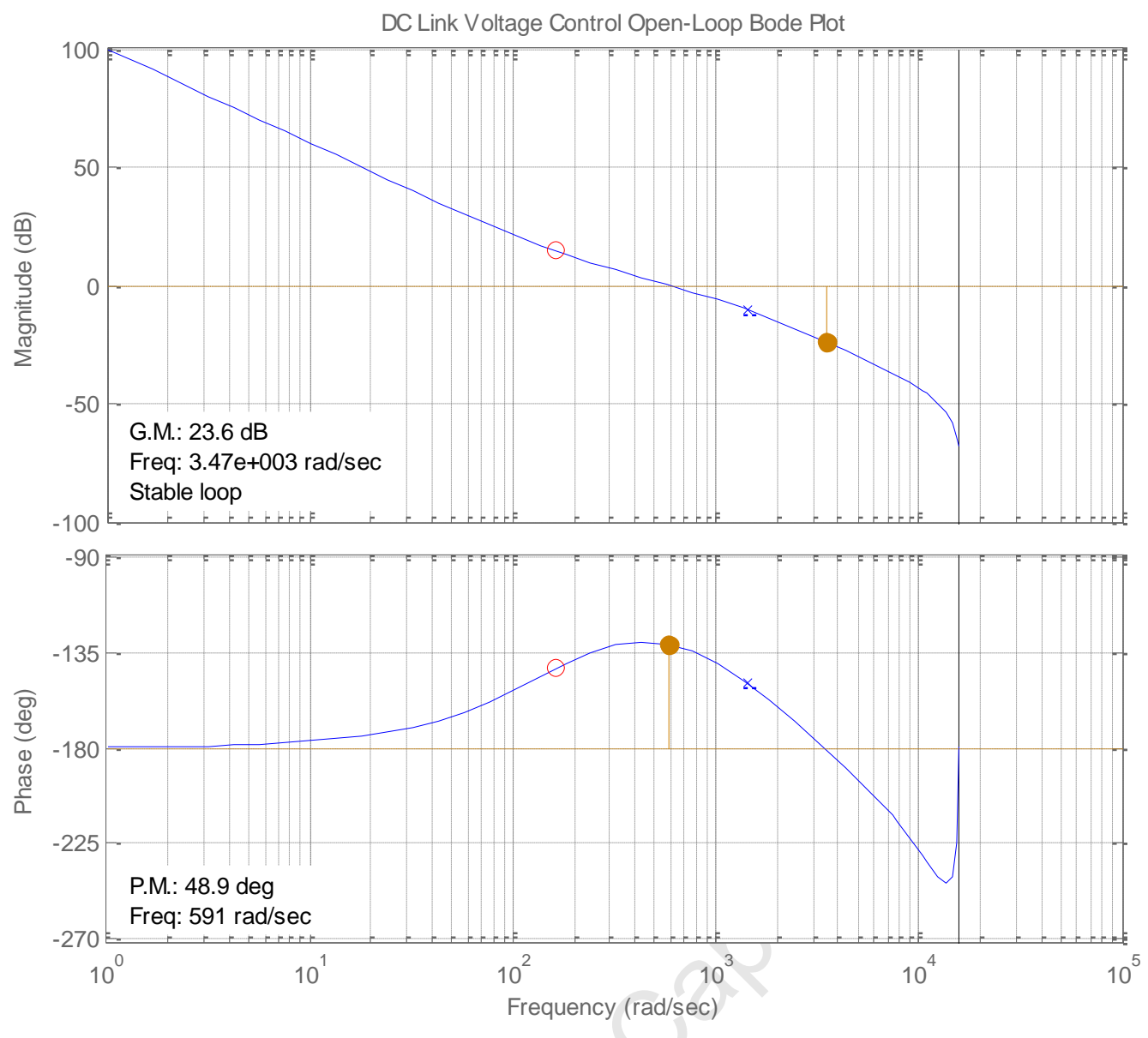

Figure 54: DC Link Voltage Loop Bode Plot

Similar to the speed loop of the machine side converter, the gains for the voltage loop was required to be altered in the experimental setup. This was due to the noise that was introduced into the system by the DSpace kit which is discussed further in section 6.5. The noise caused the system to overcompensate and hit the upper and lower saturation limits of the control. The controllers gain values were again decreased the to:

$$
\begin{gathered}
K_{p}=0.3 \\
T_{i}=0.06 \\
K_{i}=5
\end{gathered}
$$




\subsubsection{Integrator Anti-windup}

In any control system, a control output can saturate due to the physical limitations of the system. For example, in the outer speed loop for vector control of a PMSM, the output of the controller needs to be limited so that the current reference does not exceed the rating of the converter components or machine. Similarly, the output of the inner current loop controller needs to be limited so that the reference voltage does not increase the modulation index beyond unity.

If the controller output is saturated and the error signal does not decrease, the integrator output will keep growing (wind up) until the reference is surpassed. Only when the sign of the error changes, will the integrator output start to decrease. This can result in a very large overshoot as the integrator unwinds, thereby causing a poor transient response and possibly instability [44].

To solve the problem, an anti-windup circuit is required to be implemented. In digital systems this can be done by turning the integrator off when the output of the controller saturates or setting the integral gain to zero [44].

Figure 55 shows the effects of anti-windup on the controller output and the output of the loop when a step response occurs.

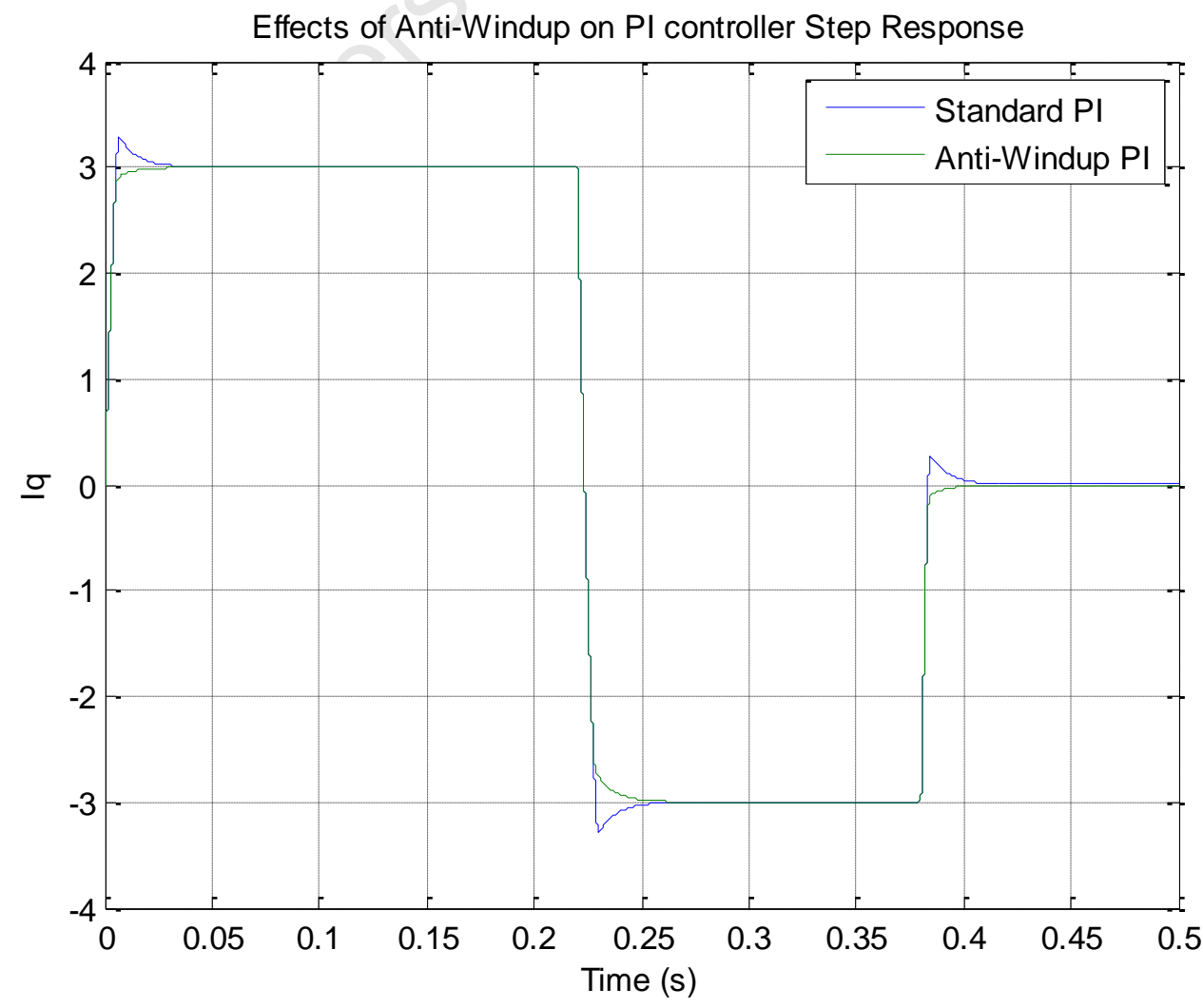

Figure 55: Standard PI and Anti-Windup PI Controller Responses 


\subsection{Conclusion}

This chapter presented two control techniques that were used to design the system controller gains. The generator-side and grid-side of the converter each have two control loops, an inner current loop and an outer speed/voltage loop. Each individual loop was modelled in this chapter by means of a block diagram and then by a transfer function. Each loop has its own controller. The effect of the designed controllers gains were verified by investigating the open-loop root locus and bode plot diagrams. Additionally, a step by step method of determining LCL filter values was presented and used to determine the LCL filter component values. 


\section{CHAPTER 5}

\section{SIMULATIONS}

All of the models that are to be implemented in the laboratory are first tested through simulation in MATLAB Simulink. The simulations are designed to resemble the experimental setup as closely as possible. The turbine emulator is investigated first, then the generator and grid side converters. The operation of the grid side converter with an unbalanced supply is also investigated.

\subsection{Turbine Emulator}

The turbine emulator equations that are used for the laboratory setup are identical to the simulation equations. The simulations therefore provide the exact waveforms that will be produced in the laboratory. Each of the real turbine effects are investigated in turn in the following sections.

\subsubsection{Wind Shear}

The wind shear effect was implemented using equation 2.20. A sinusoidal output is expected due to the second term in the equation. The effect of wind shear on the normalised torque reference for the turbine emulator is presented in Figure 56 below. The wind shear does not have a big effect on the overall torque produced but causes a sinusoidal deviation of the reference between $0.4-0.5 \%$. 


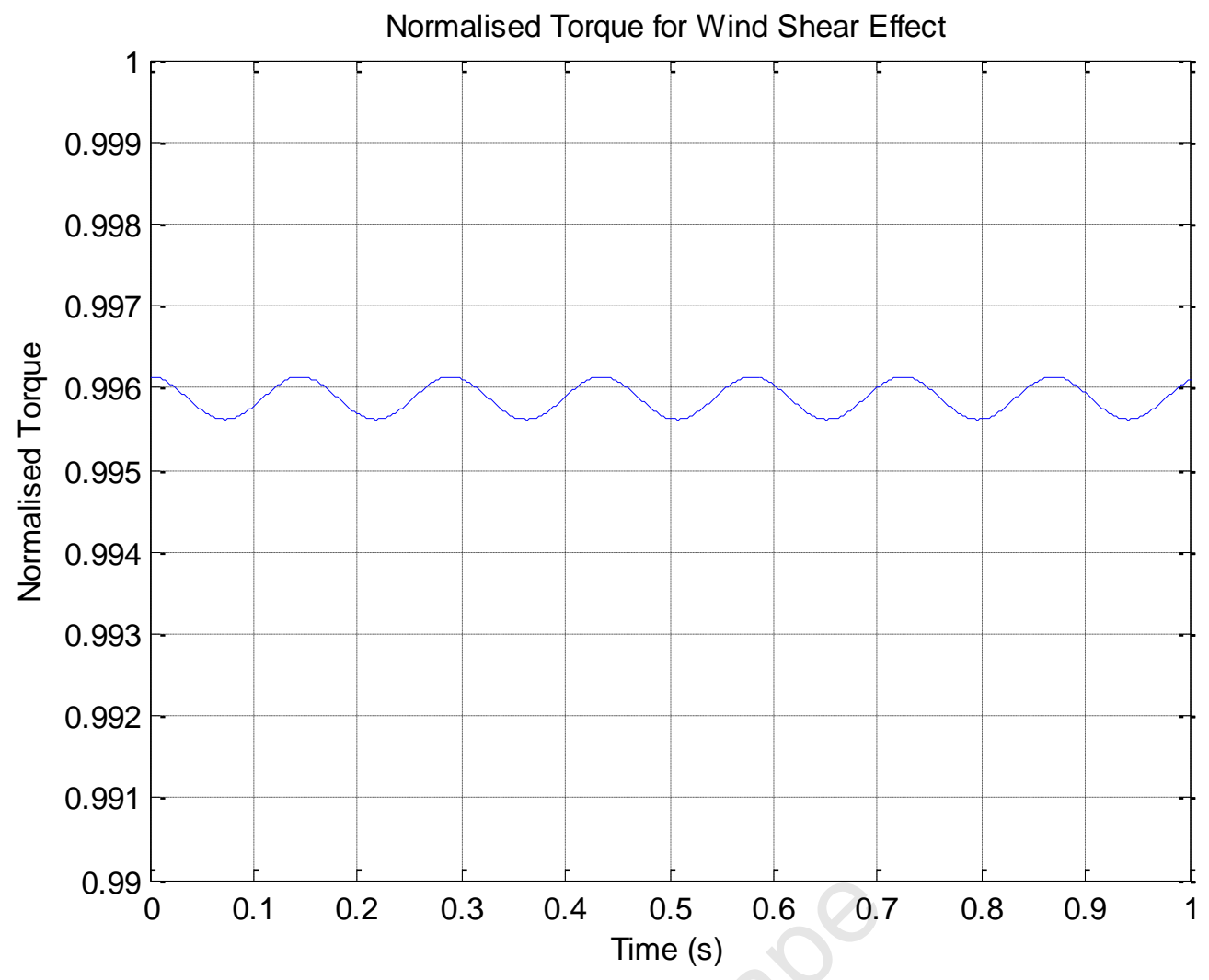

Figure 56: Simulated Normalised Torque Reference for Turbine Emulator with Wind Shear

\subsubsection{Tower Shadow}

Tower shadow has a more pronounced effect on the turbine torque than the previously investigated wind shear effect. The normalised torque reference is determined using equation 2.2.1 and the resulting waveform is presented in Figure 57. Deviations of up to $6 \%$ are produced each time a blade passes the tower, which can affect the overall performance of the system. 


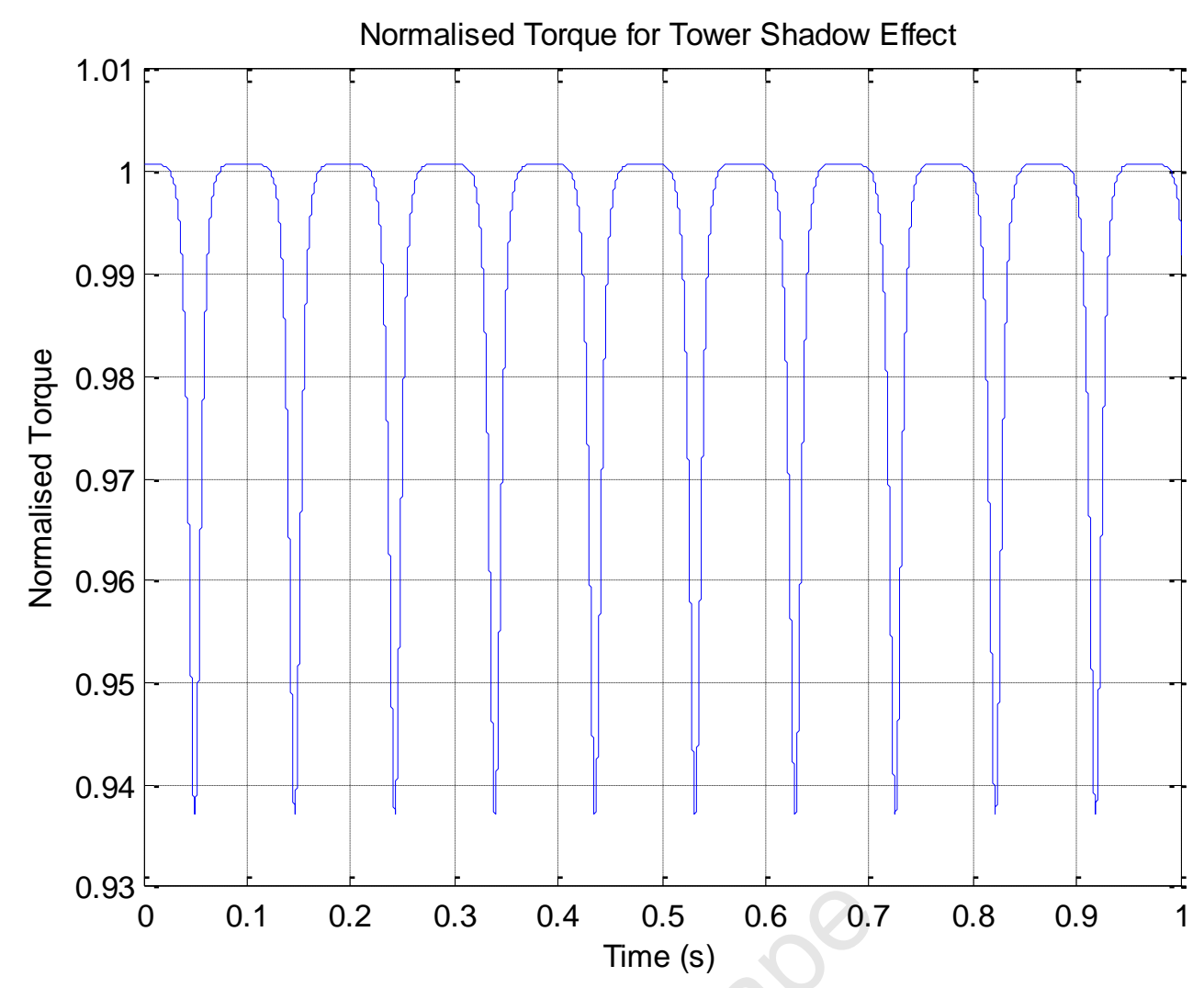

Figure 57: Simulated Normalised Torque for Tower Shadow

\subsubsection{Turbulence}

The turbulence model simulation is presented in Figure 58 for an average wind speed of $7 \mathrm{~m} / \mathrm{s}$. It can be seen that the wind speed varies between $4-9 \mathrm{~m} / \mathrm{s}$ during the simulated period. The turbulence is introduced by passing band-limited white noise through the shaping filter of equation 2.13 . The fairly rapid variation in wind speed will be used as a test to analyse the effectiveness of the system control. 


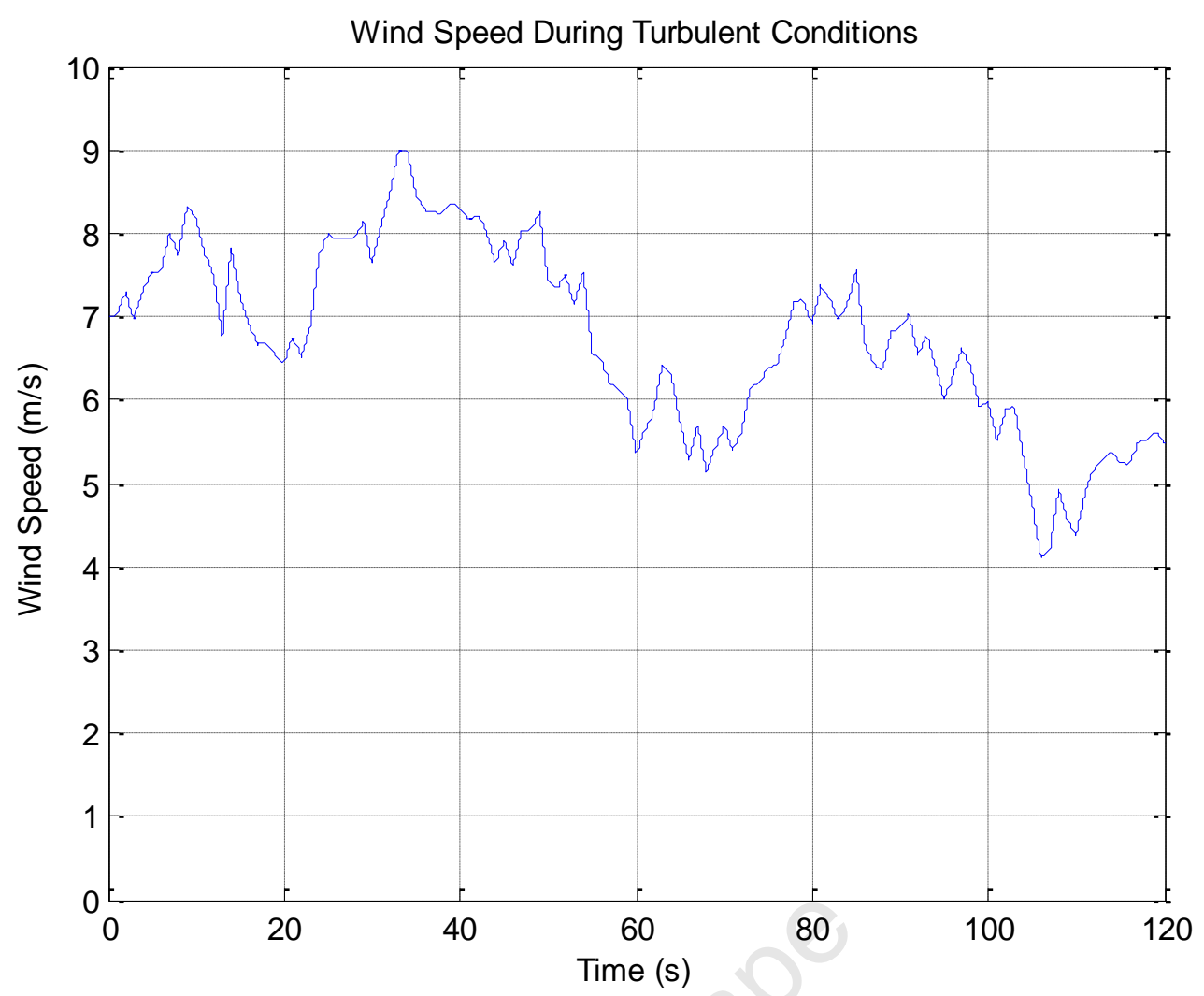

Figure 58: Simulated Turbulent Wind Speed

\subsection{Generator Side}

The generator side converter was simulated to evaluate its performance before prototyping. The generator side simulation excludes the grid side of the system and assumes a constant DC link voltage of $650 \mathrm{~V}$ is available. The step response tests are performed to confirm the correct operation of the system and are implemented using the same controller gains that are used in the laboratory setup. The current loop controller gains are as designed in chapter 4 whilst the speed loop gain was determined by trial and error in the laboratory. The simulation responses are used to prove the accuracy of the simulated models.

\subsubsection{Current Step Response}

The system in Figure 42 was simulated by performing a blocked rotor test and stepping the current set point, $I_{q}$. The blocked rotor test decouples the voltage equations 3.10 and 3.11 by removing the speed terms. A PMSM block from the MATLAB/Simulink SimPowerSystems block set was used to simulate the generator. The blocked rotor test was implemented by 
setting the mechanical speed of the PMSM to zero. The quadrature current was then stepped to a value of $-5 \mathrm{~A}$ and the observed response is illustrated in Figure 59 . The settling time is within $2 \mathrm{~ms}$ with slight overshoot present. An oscillation is present once the set point is reached which could not be removed with reasonable values of the controller gains. The step response using transfer functions is shown in Figure 45 and exhibits no oscillatory behaviour. The oscillations in this response are then due to some characteristic in the PMSM block. The response settling time is good considering the speed loop will have a much larger time constant.

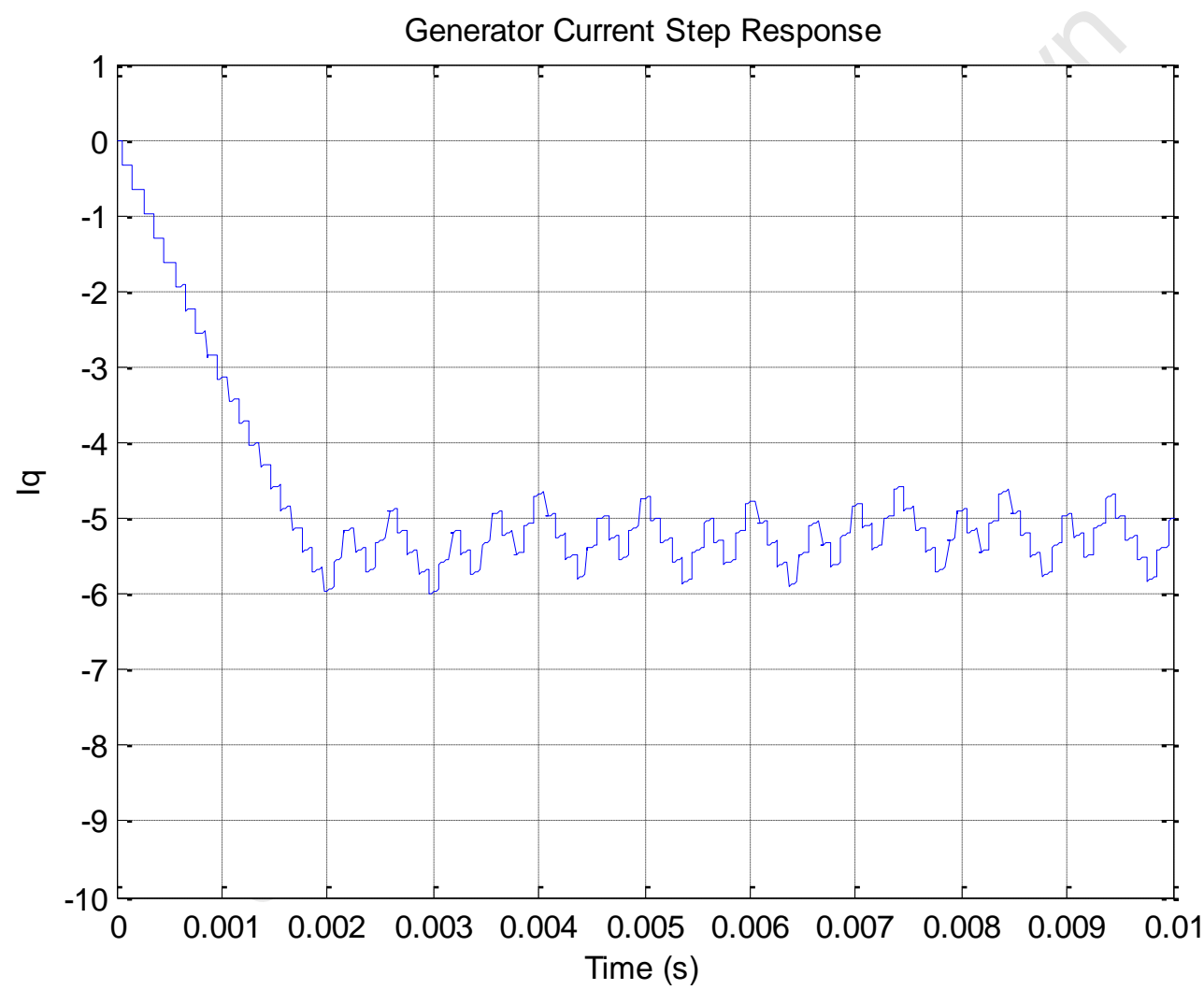

Figure 59: Simulated PMSG Current Step Response

\subsubsection{Speed Step}

Figure 46 represents the system during the speed response simulation. The speed step response of the generator is performed by selecting a constant value for input torque of the PMSM block and the speed set point is changed from 5 to $10 \mathrm{rad} / \mathrm{s}$. A settling time of $700 \mathrm{~ms}$ and an overshoot of approximately $15 \%$ is observed in Figure 60 below. The poles of this control loop are not located in the same position as Figure 47 because the controller gains 
were changed to compensate for the variable delay in the experimental speed calculation. This alters the settling time and overshoot of the step response. The settling time is reasonable when comparing it to the variation in wind speeds which is on a time scales of seconds rather than milliseconds. The ability of the generator to track the reference speed, obtained from the MPPT algorithm during turbulence, is investigated in section 5.5.

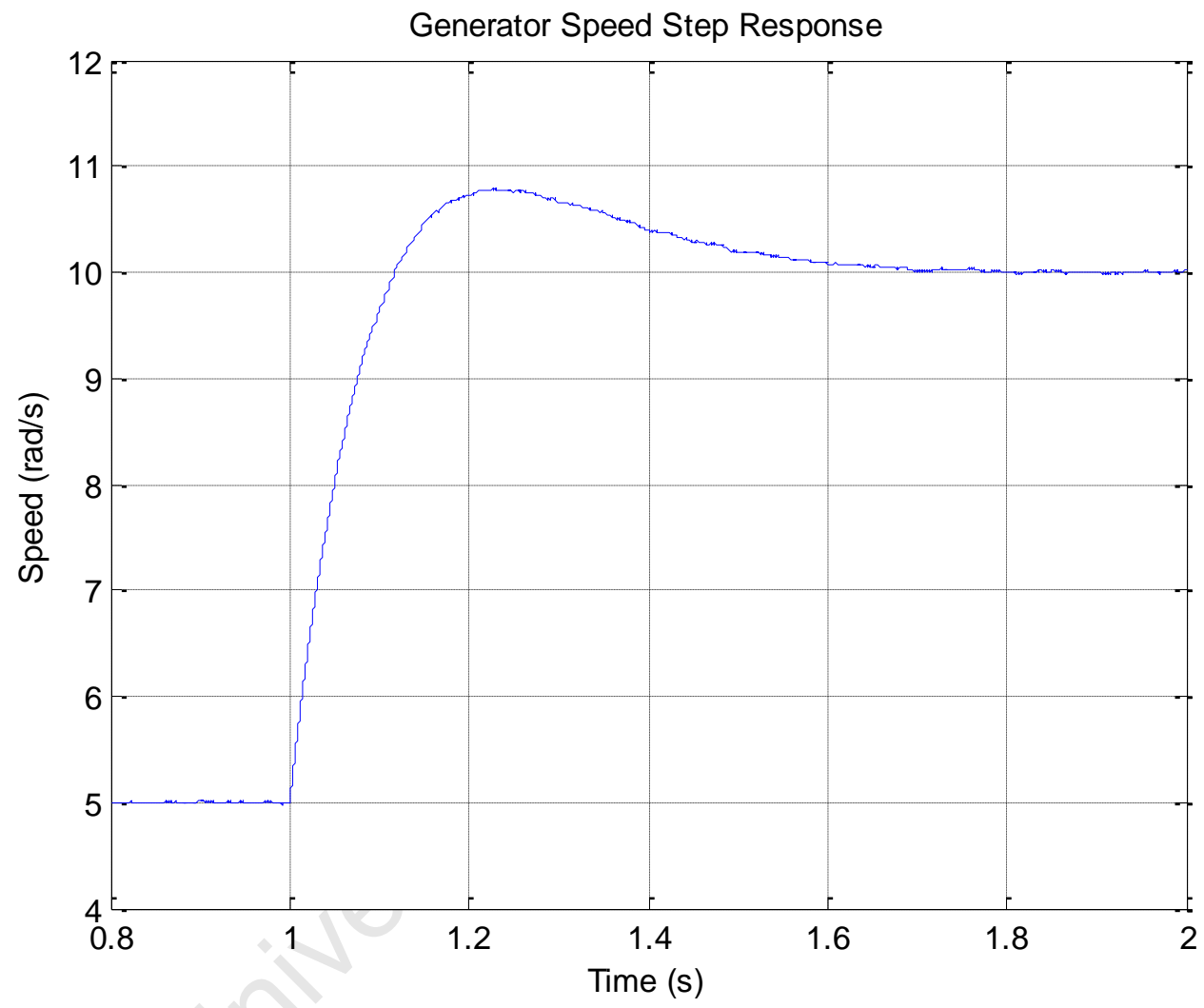

Figure 60: Simulated PMSG Speed Step Response

\subsection{Grid Side}

The grid side converter is simulated in this section. Its performance is decoupled from the generator side by supplying the DC bus with a constant DC current, thereby eliminating the need of the generator side of the system. The step response tests are performed for the same reasons discussed in section 5.2. The current loop uses the designed controller values determined in chapter 4 and the voltage loop uses values that were determined in the lab due to the large amount of noise on the DC Bus signal. 


\subsubsection{Current Step Response}

The step response is performed on the system of Figure 49. The DC link voltage control is removed in order to perform the current response test and the DC link capacitor is replaced with a DC voltage source to prevent the voltage from becoming too low. The current step was executed by changing the set point of the direct current component $I_{q}$ from $0 \mathrm{~A}$ to $-5 \mathrm{~A}$. Figure 61 shows the resulting response, which has a settling time of $5 \mathrm{~ms}$ with no overshoot. The settling time is good and has a much smaller time constant than the outer voltage loop in section 5.3.2.

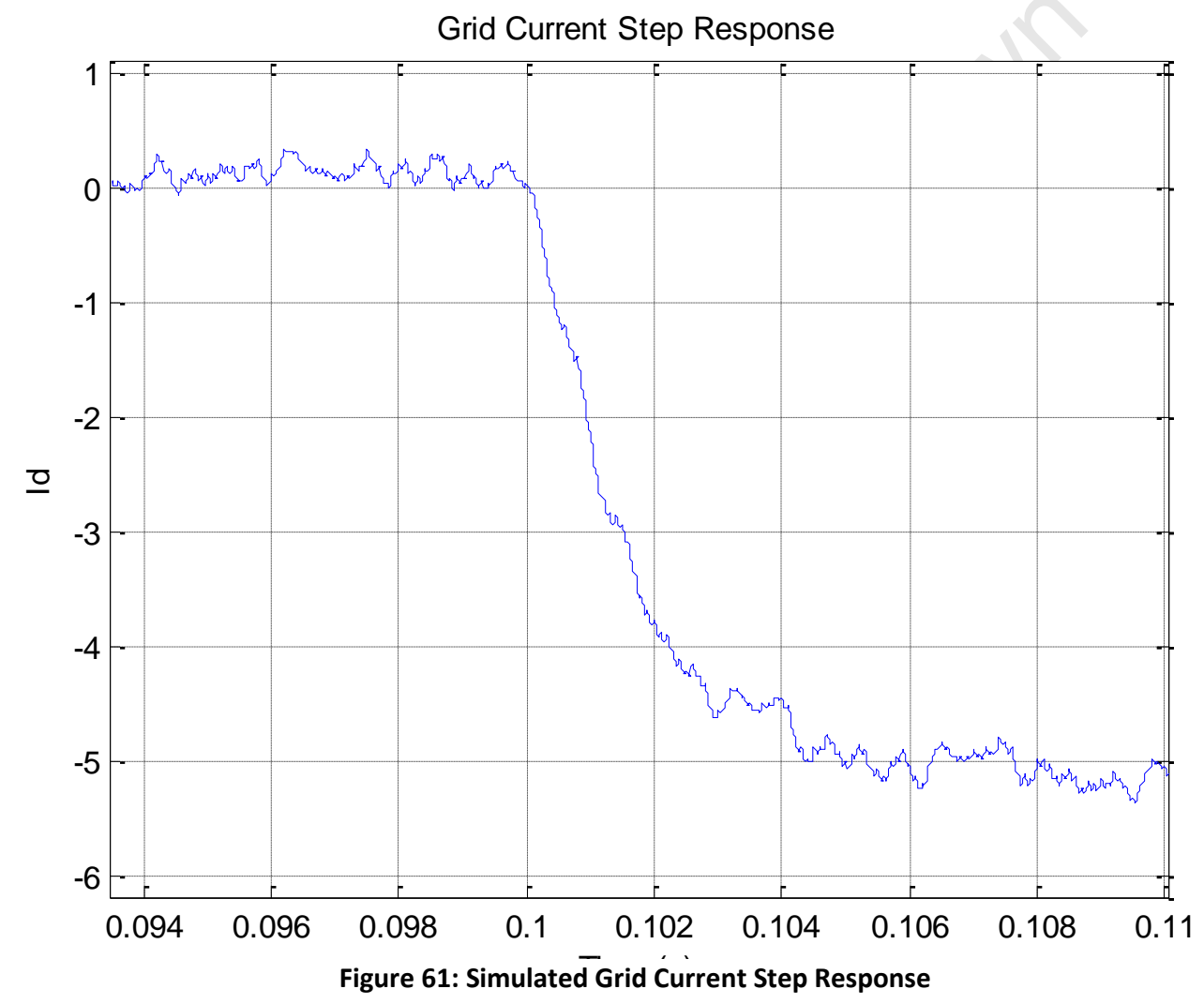

\subsubsection{Voltage Step}

The voltage step response is based on the system presented in Figure 52. The DC link capacitor was added back to the model to test the voltage step response. A set point change from $600 \mathrm{~V}$ to $640 \mathrm{~V}$ was performed and the results are illustrated in Figure 62 which indicates a settling time of $200 \mathrm{~ms}$ and an overshoot of approximately $17 \%$. Similarly to the generator speed loop, the controller gains have been altered changing the response slightly. The settling time has a smaller time constant than both the wind turbulence and generator 
speed response which suggests that it will be able to keep the DC link voltage constant throughout operation.

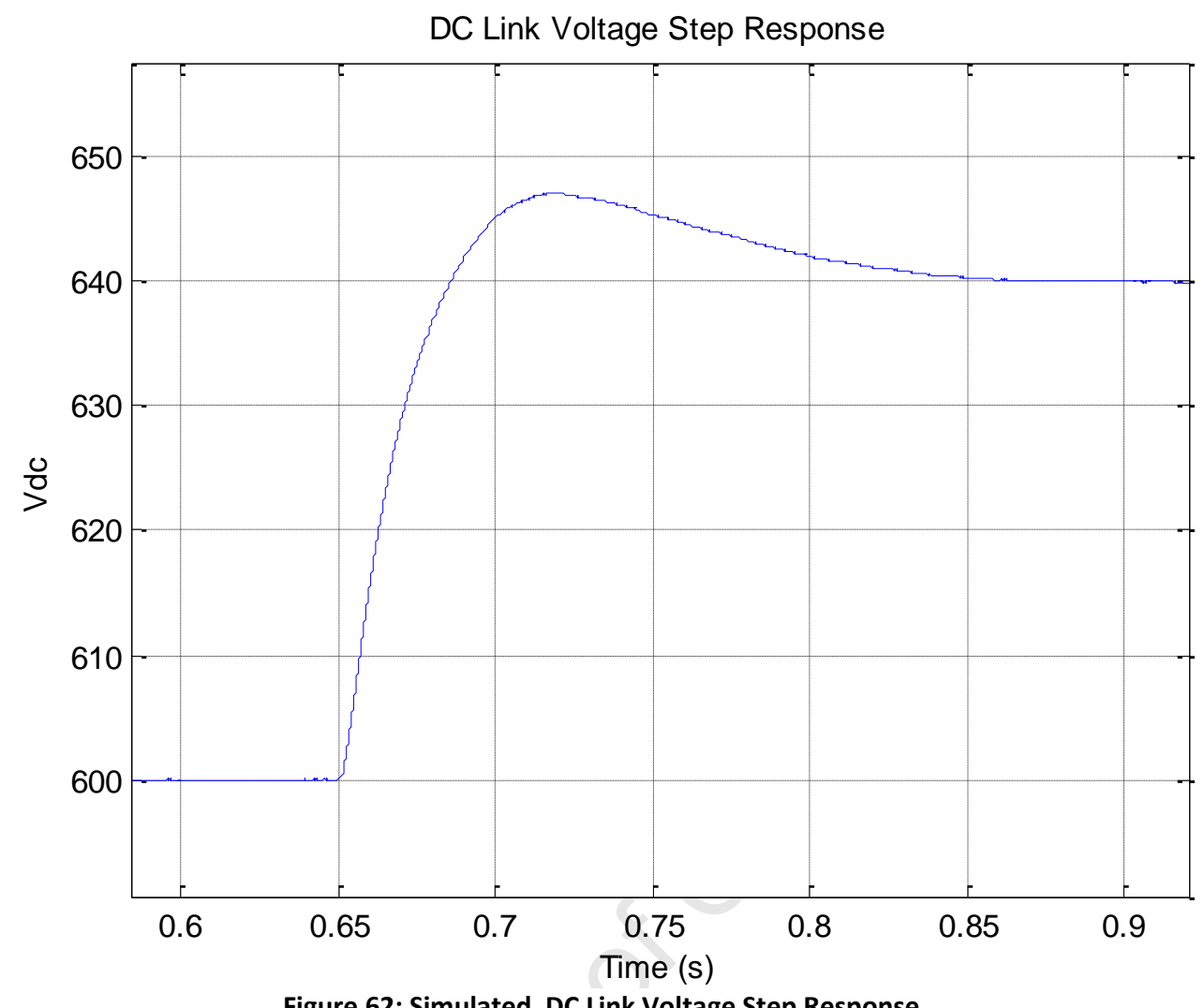

Figure 62: Simulated DC Link Voltage Step Response

\subsection{Simulated Turbine Effects}

This section investigates the effect that specific turbine effects in section 5.1 have on the generator shaft torque, speed and power. The simulation included only the generator side of the system and the grid side was simulated using a DC voltage source.

\subsubsection{Simulated Effect of Wind Shear on Generator}

The effect of wind shear on the generator parameters is illustrated in Figure 63 below. Previous simulations in section 5.1 indicate that wind shear does not have a large effect on the turbine torque, therefore only a slight sinusoidal shape on the shaft power, speed and torque is expected. The results confirm the expectations and only a small variation of the shaft power is visible. The torque and speed variations are on a much smaller scale than the power. 


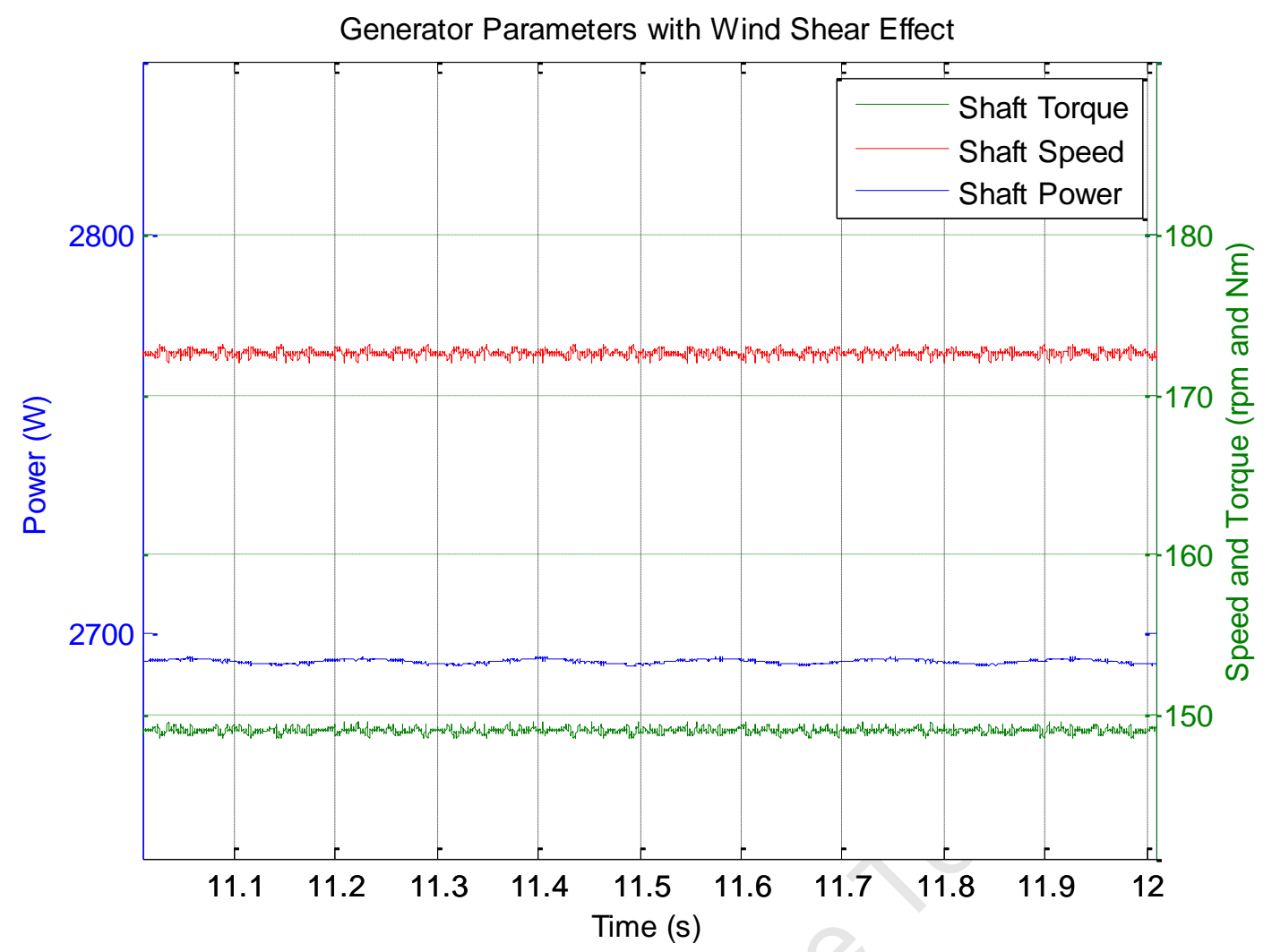

Figure 63: Simulated Effect of Wind Shear on Generator

\subsubsection{Simulated Effect of Tower Shadow on Generator}

Tower shadow is expected to have a much larger effect on the generator parameters than wind shear as discussed in section 5.1. Figure 64 shows the generator parameters under the influence of the tower shadow effect. The shaft torque dips occur three time per revolution as expected and have a magnitude of $4 \%$. The dips in the generator shaft speed and power correspond to the dips in the torque. The speed dips are smaller in magnitude compared to the torque dips because the turbine and generator have a certain amount of inertia which prevents quick changes in the generator shaft speed. 


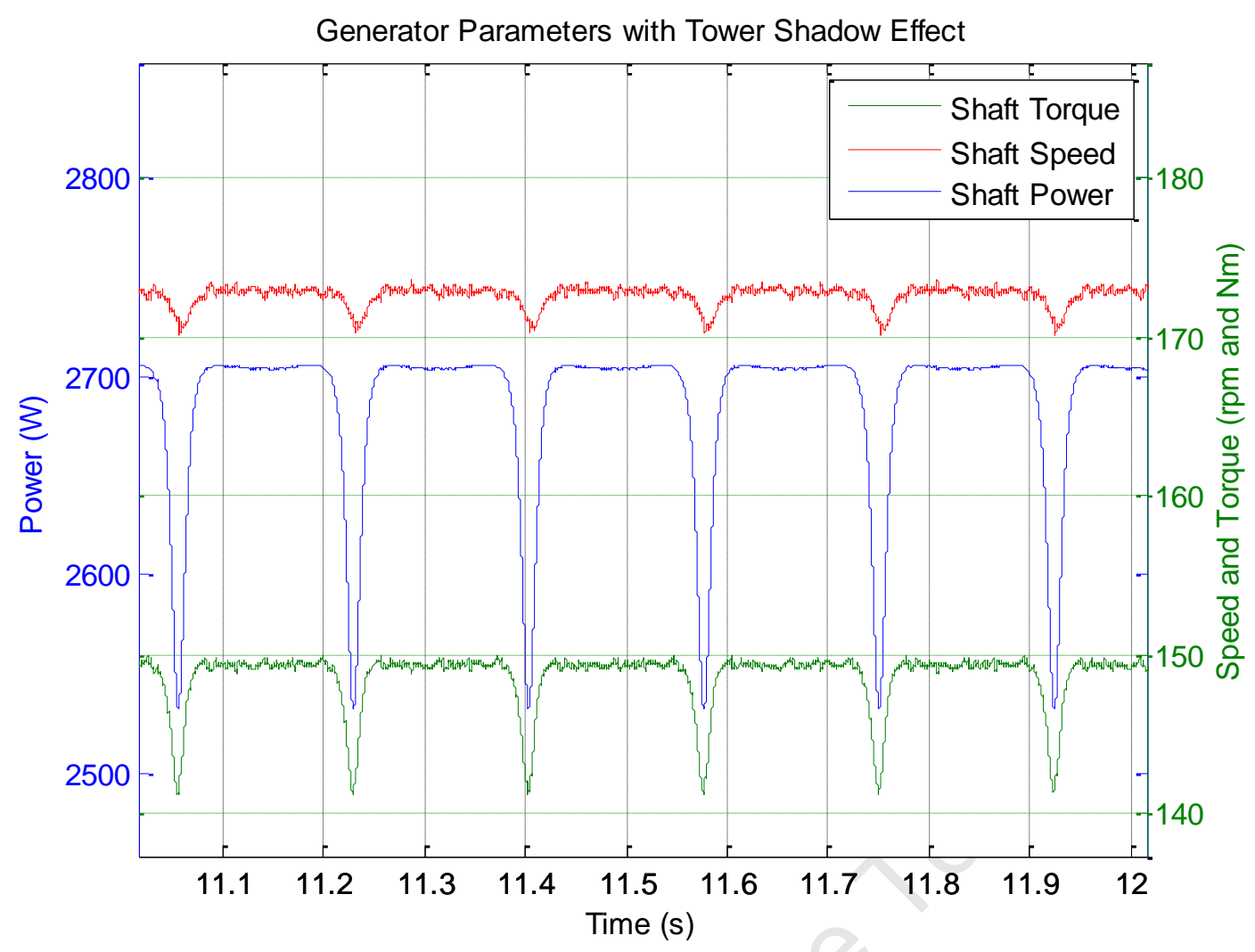

Figure 64: Simulated Effect of Tower Shadow on Generator

\subsubsection{Simulated Effect on Generator with all Turbine Effects}

The combination of tower shadow and wind shear is illustrated in Figure 65. It is expected that the results would be similar to having only the tower shadow effect. This holds true but the wind shear effect can be seen on the shaft power curve. Just before and after the tower shadow dips occur, the power increases, suggesting that the wind shear provides the most torque when the a blade passes the tower or alternatively when two of the blades are $60^{\circ}$ from vertical. 


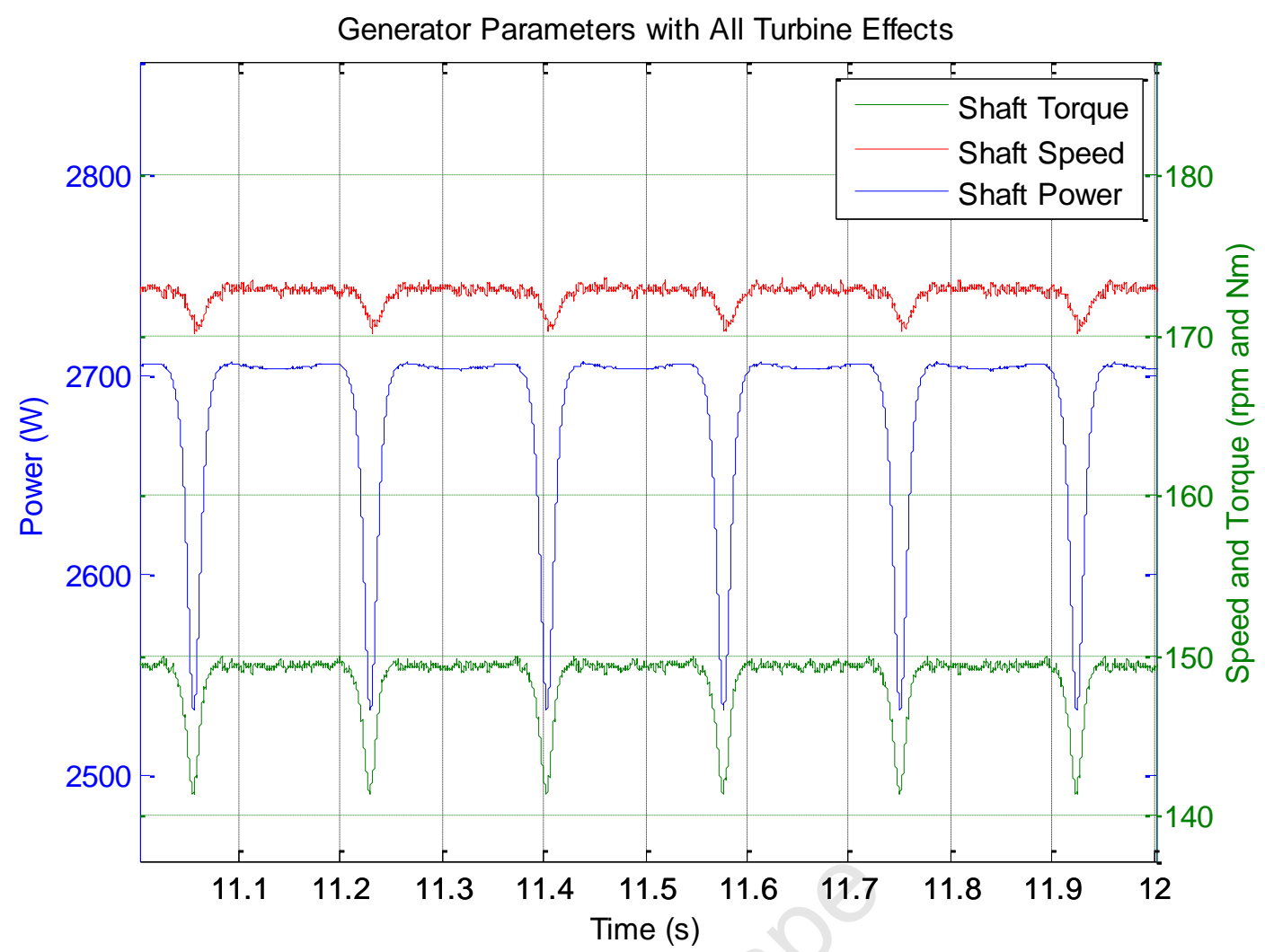

Figure 65: Simulated Generator Parameters for All Turbine Effects

\subsection{Control Effectiveness Under Unbalanced Operating}

\section{Conditions}

This thesis only investigates the effects of magnitude unbalance, and does not include phase unbalance. The effect of unbalance on the system and the ability of the control techniques to operate under the non-ideal conditions are investigated in this section. A voltage unbalance could not be created in simulation due to limitations experienced with Simulink. Resistors were added to each phase between the grid filter and the grid itself in order to simulate unbalanced grid conditions. The conditions that were investigated include balanced grid voltages and a type B dip. Each condition is tested with standard vector control as well as dual vector current control and the results are compared. The simulations for the two control techniques where run under different loads and does not affect the results in any way. 


\subsubsection{Standard Vector Control}

Standard Vector Control is used and UPF operation is ensured by keeping $I_{q}$ at zero. The control is then tested on different unbalance conditions. A block diagram for this control technique is provided in Figure 23.

\subsubsection{Balanced Grid Voltages}

Figure 66 illustrates the grid phase currents under balanced grid voltage conditions when the resistor values are all equal. The phase currents are equal and the $d q$ currents have expected constant values as shown in Figure 67.

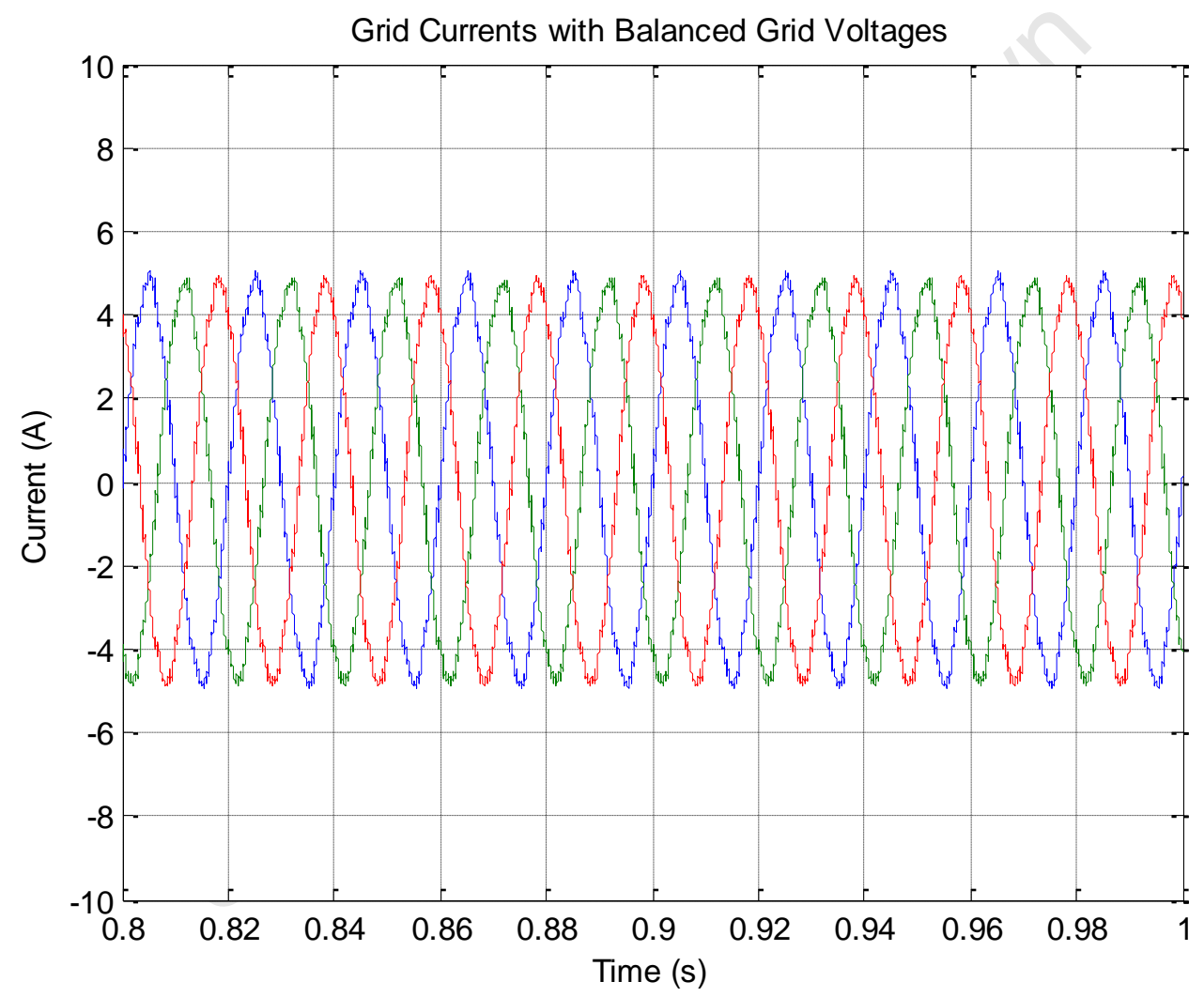

Figure 66: Simulated Grid Current with Balanced Grid Voltages 


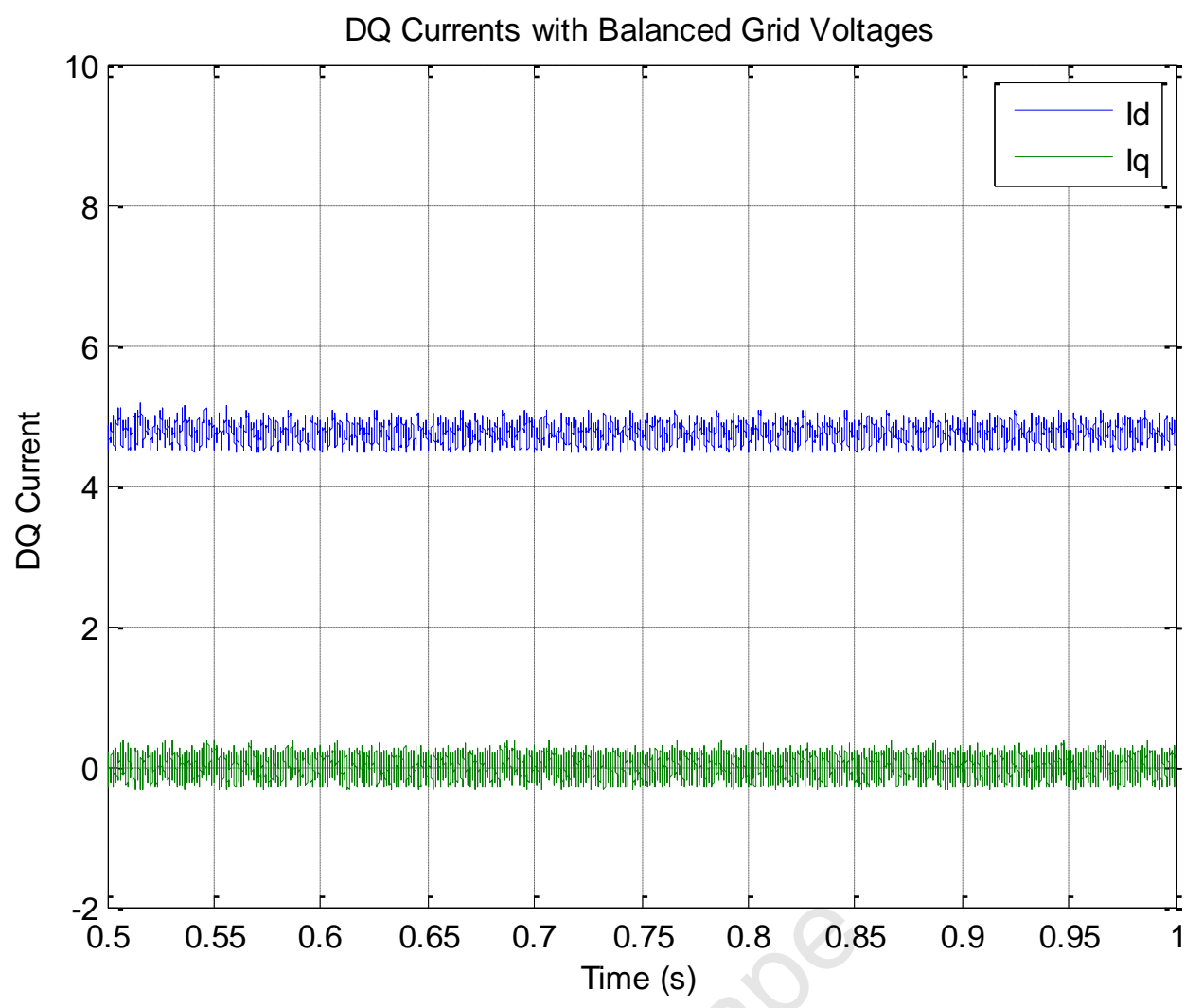

Figure 67: Simulated DQ Currents with Balanced Grid Voltages

\subsubsection{Type B Dip}

A type B dip is introduced to the system by reducing the value of one of the phase resistors. This has the effect of reducing the magnitude of one phase voltage, whilst maintaining the other two at nominal values. The angles between the phase voltages are maintained, thereby allowing magnitude unbalance to be implemented. The resulting grid currents are not balanced as can be seen in Figure 68 where one of the phases has a greater magnitude than the other two. This is due to that phase having less impedance than the others. Figure 69 presents the $d q$ currents which have a $100 \mathrm{~Hz}$ oscillation superimposed on them, due to the negative sequence components which have been introduced to the system by the unbalance. The $100 \mathrm{~Hz}$ component is expected and is discussed in section 3.5. 


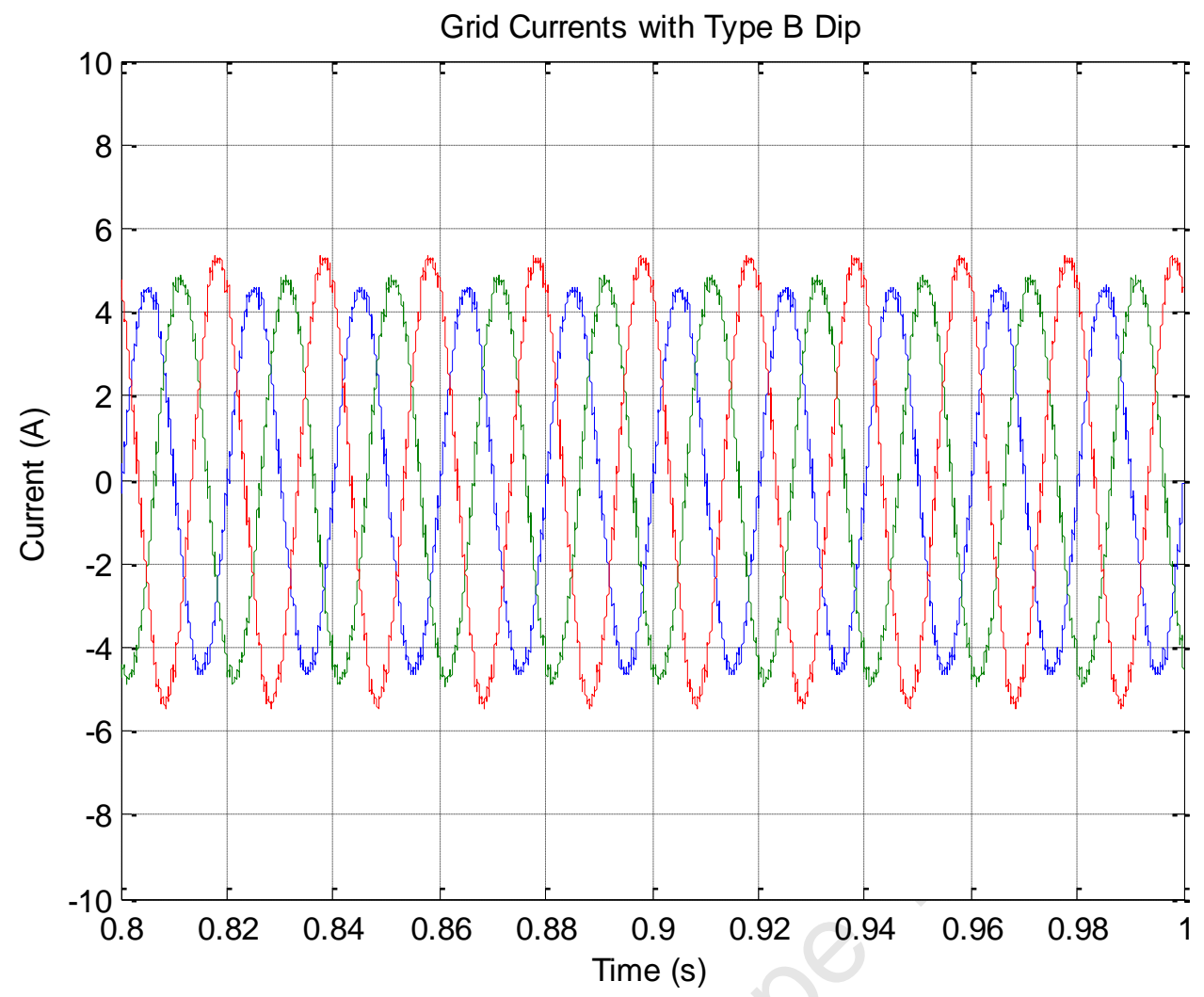

Figure 68: Simulated Grid Currents with Type B Dip

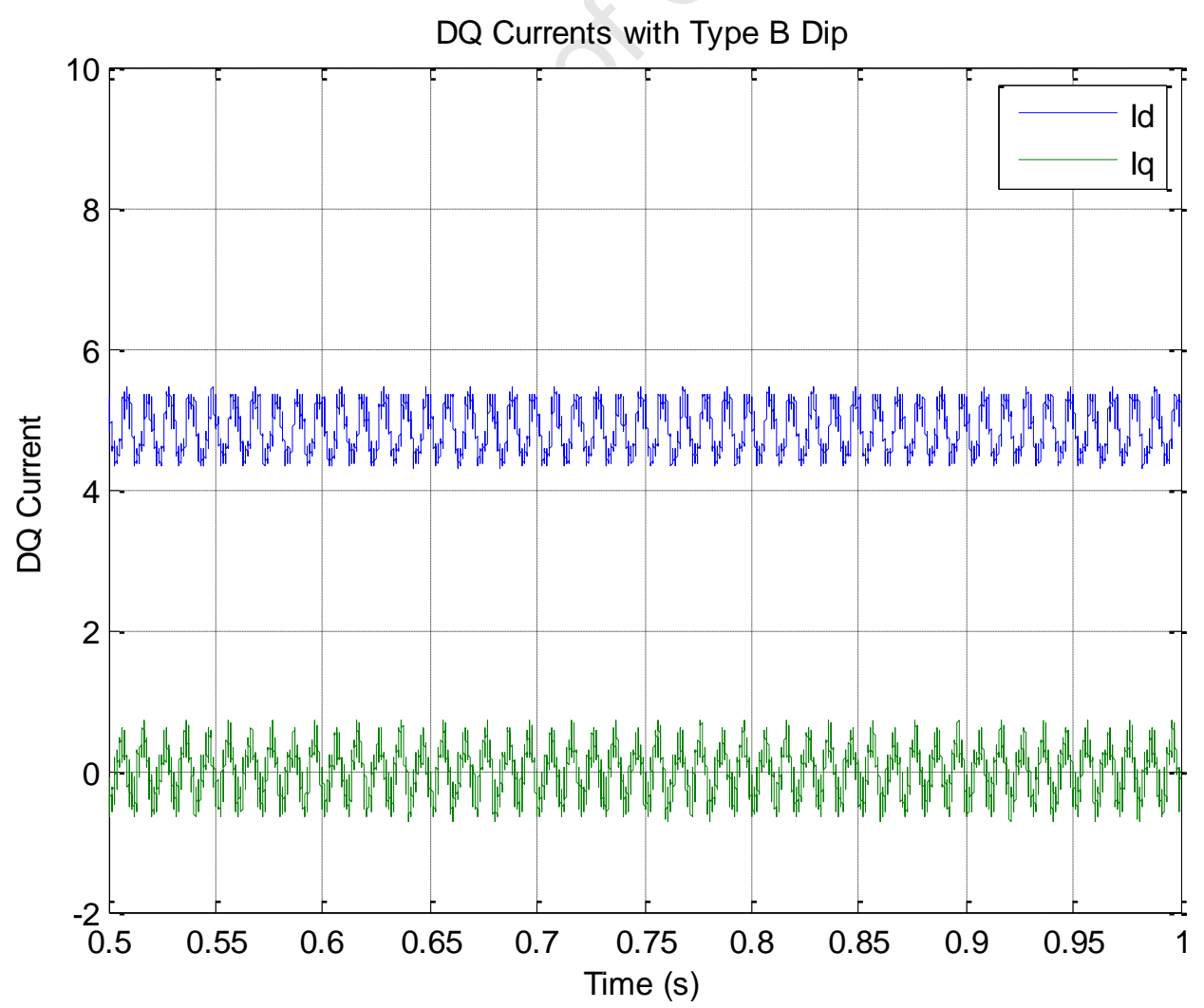

Figure 69: Simulated DQ Currents with Type B Dip 


\subsubsection{Dual Vector Control}

The Dual Vector Current Control is tested under the same conditions as above but is expected to have an improved performance because it is able to control the negative sequence currents. A block diagram which illustrates this control technique is provided in Figure 37.

\subsubsection{Balanced Grid Voltages}

Figure 70 illustrates the grid phase currents under balanced conditions, which are all equal in magnitude. Figure 71 and Figure 72 show the positive and negative sequence $d q$ currents respectively. They are all constant in nature but have a large band of distortion, which is caused by the sequence extraction method discussed in 3.5.1.2. The positive sequence $d-$ axis component $I_{d}$ is used to transfer the power to the grid and the positive sequence quadrature current as well as both the negative sequence currents components are maintained at zero by the controllers.

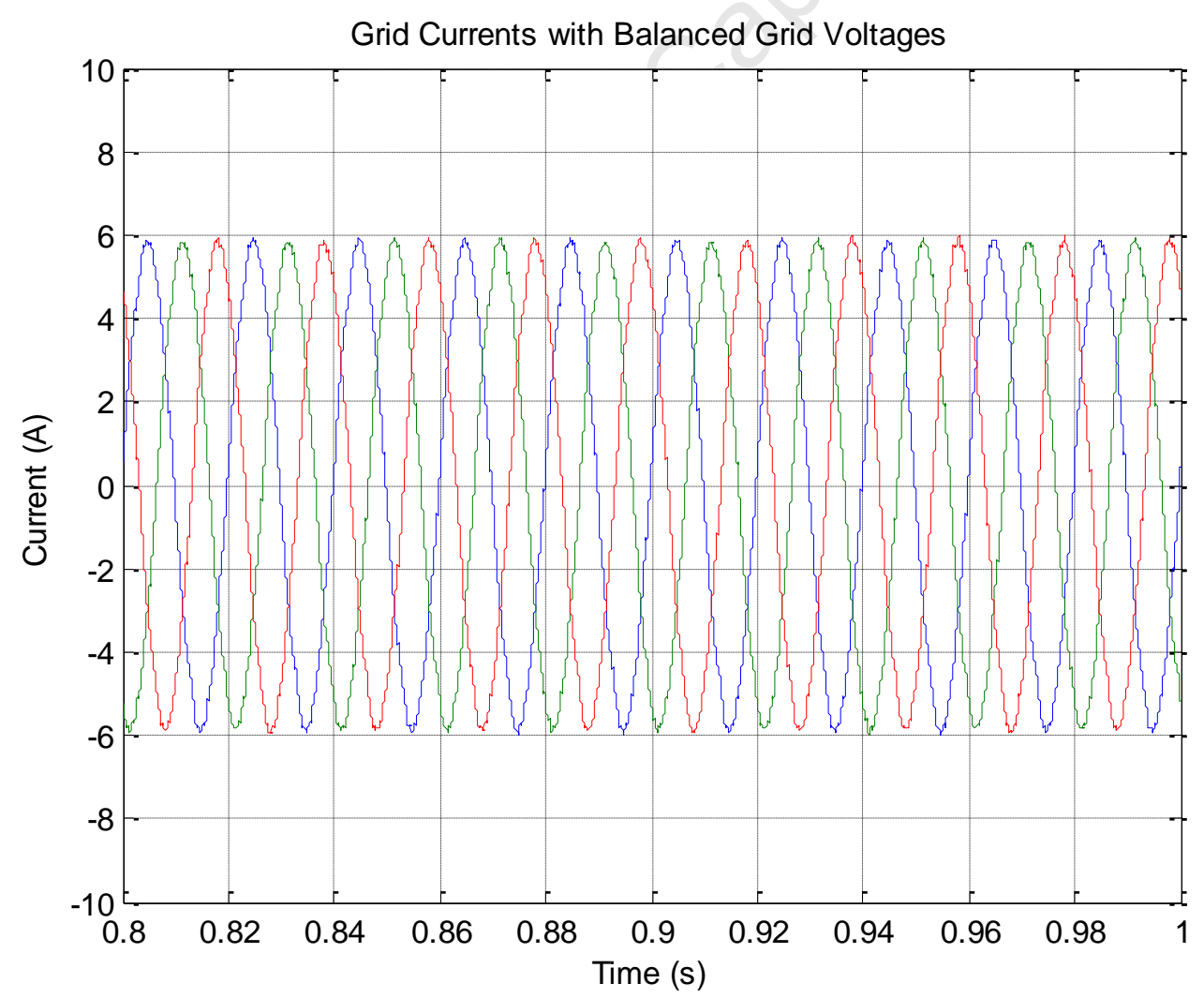

Figure 70: Simulated Dual Vector Control Grid Currents Balanced Grid Voltages 


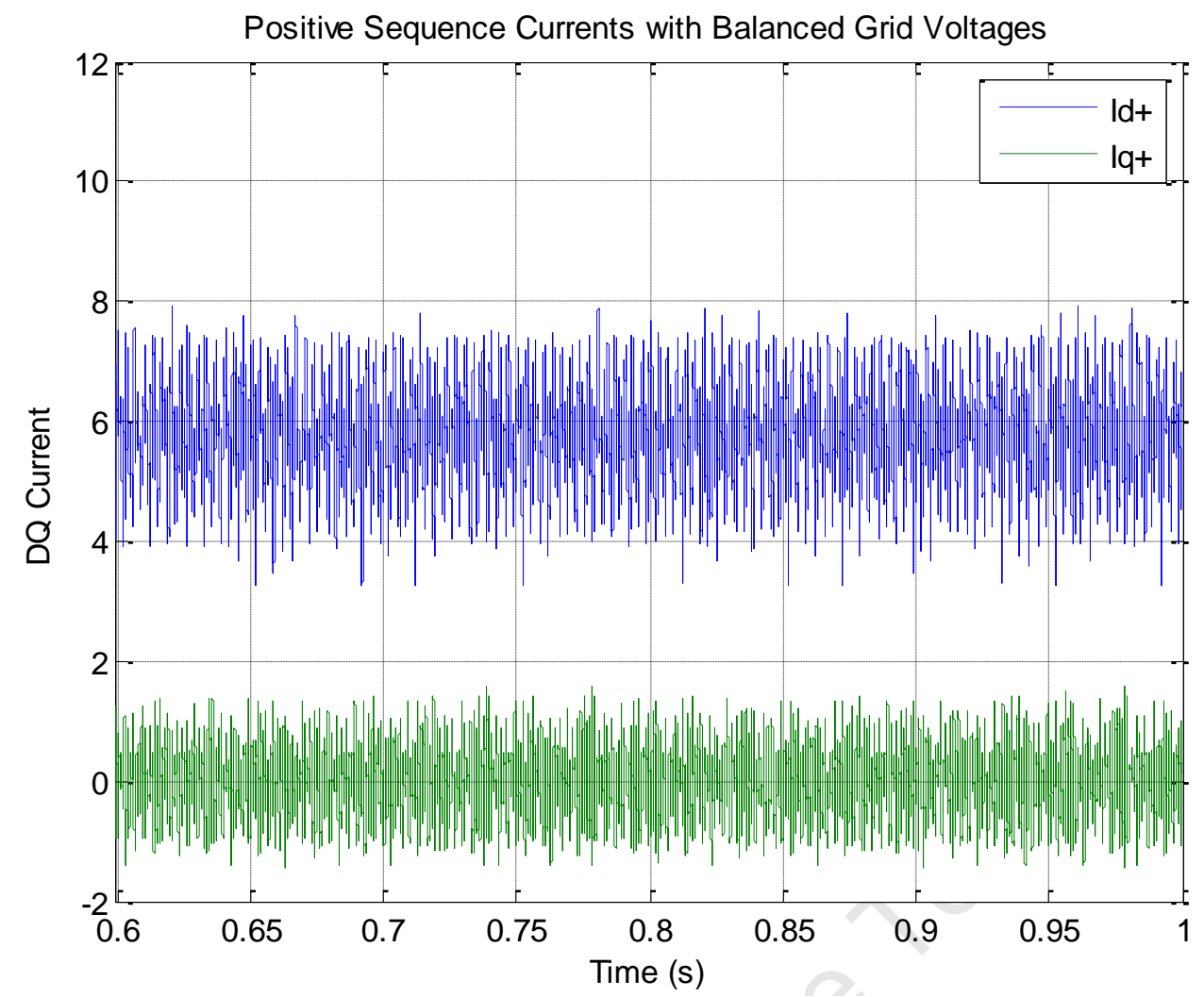

Figure 71: Simulated Dual Vector Control DQ+ Currents with Balanced Grid Voltages

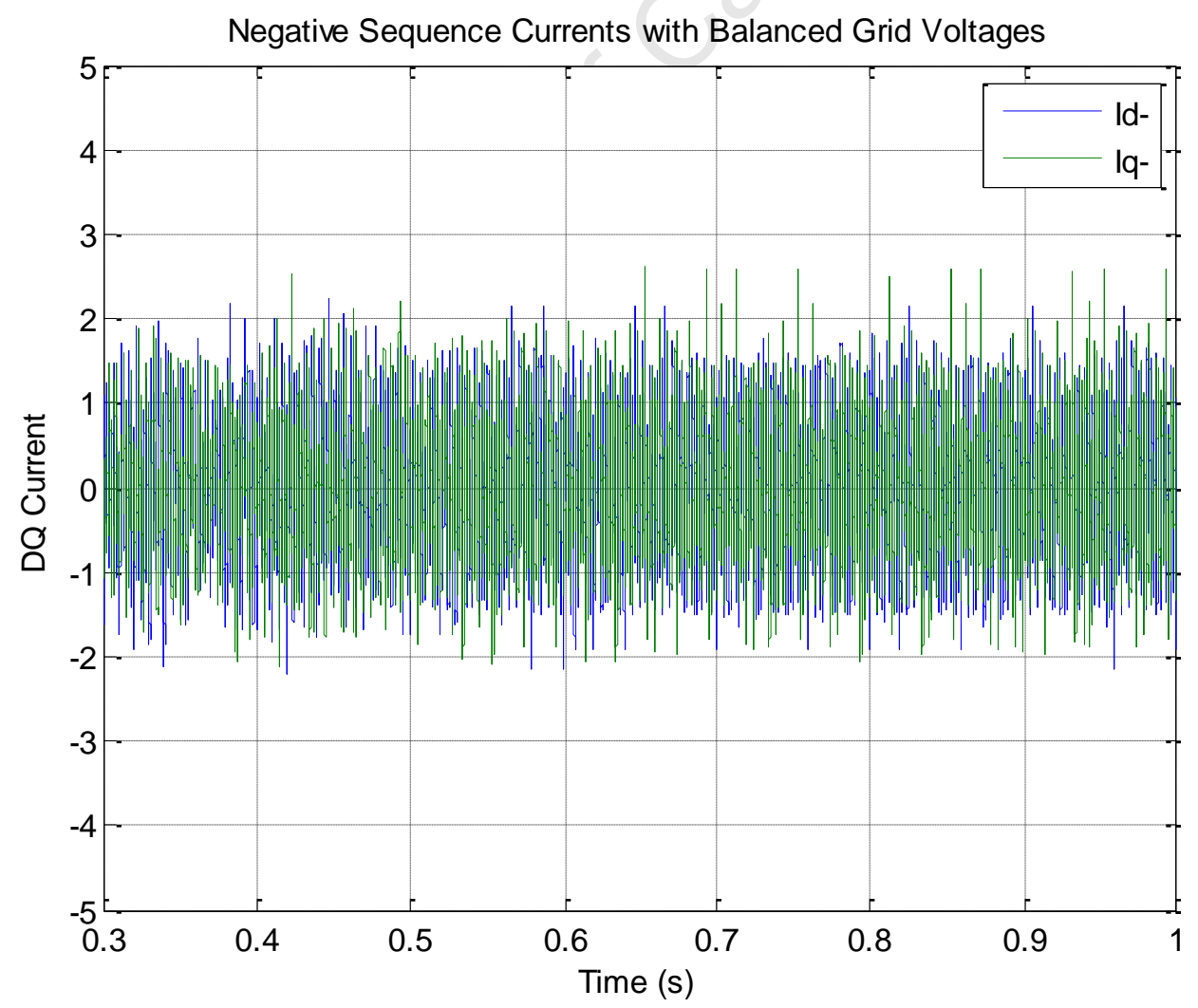

Figure 72:Simulated Dual Vector Control DQ- Currents with Balanced Grid Voltages 


\subsubsection{Type B Dip}

The type B dip is implemented as discussed in section 5.4.1.2. The grid currents are displayed in Figure 73 below and are shown to have phase currents that are equal in magnitude. Figure 74 reveals unchanged positive sequence components when compared to the balanced grid voltage situation. This can be explained by considering the $d$-axis positive and negative sequence components. When there are balanced phase voltages, the phase currents are balanced and therefore the negative sequence component $I_{d-}$ is zero. The entire $d$-axis current value then consists of $I_{d+}$. When unbalanced phase voltages are encountered, the phase currents will become unbalanced and the d-axis currents will consist of both $I_{d-}$ and a changed value of $I_{d+}$. The Dual Vector Current Control is able to force the negative sequence currents to zero thereby restoring the positive sequence currents to their original values. The negative sequence components are shown to be zero in Figure 75 , thereby balancing the grid currents.

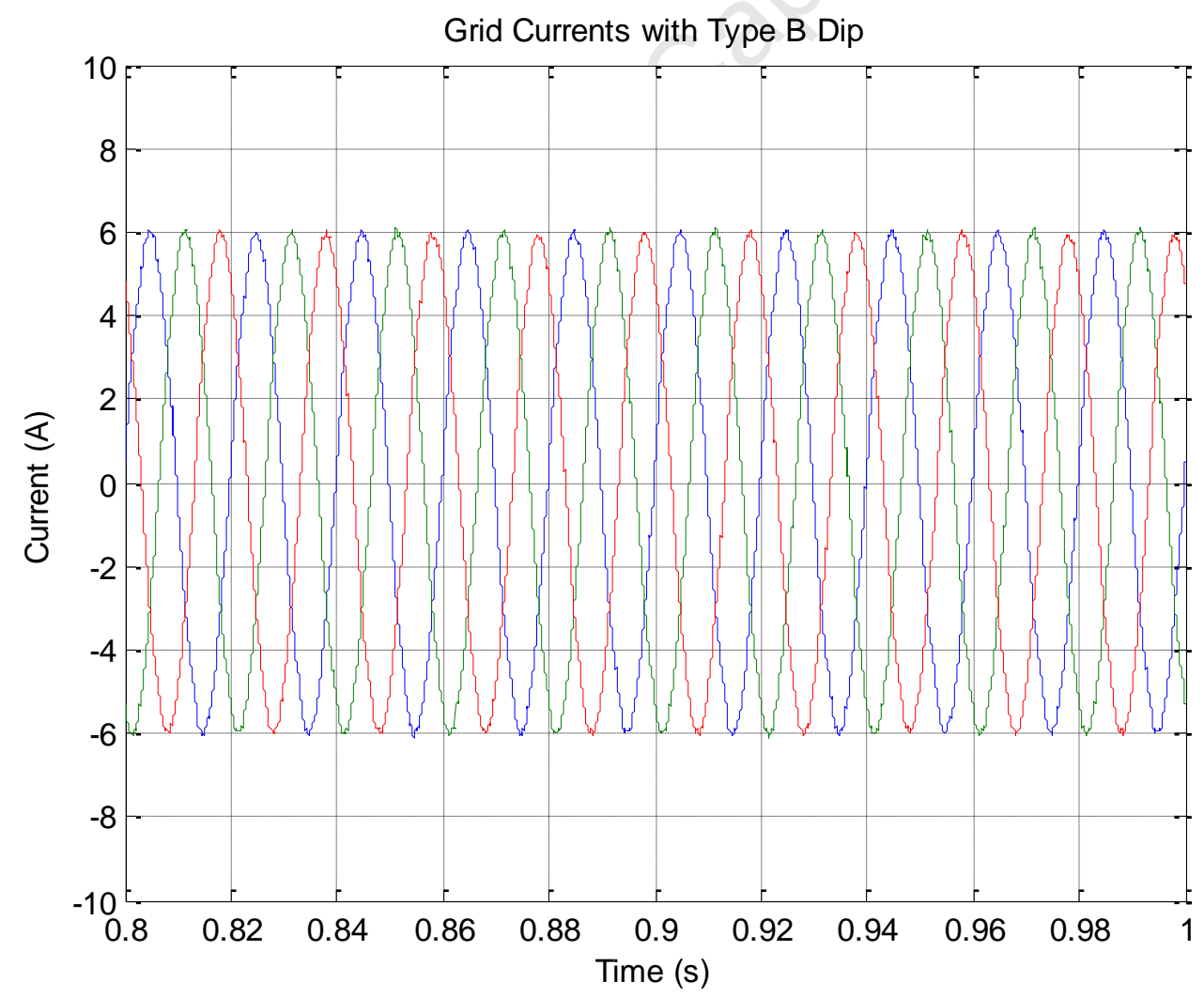

Figure 73:Simulated Dual Vector Control Grid Currents with Type B Dip 


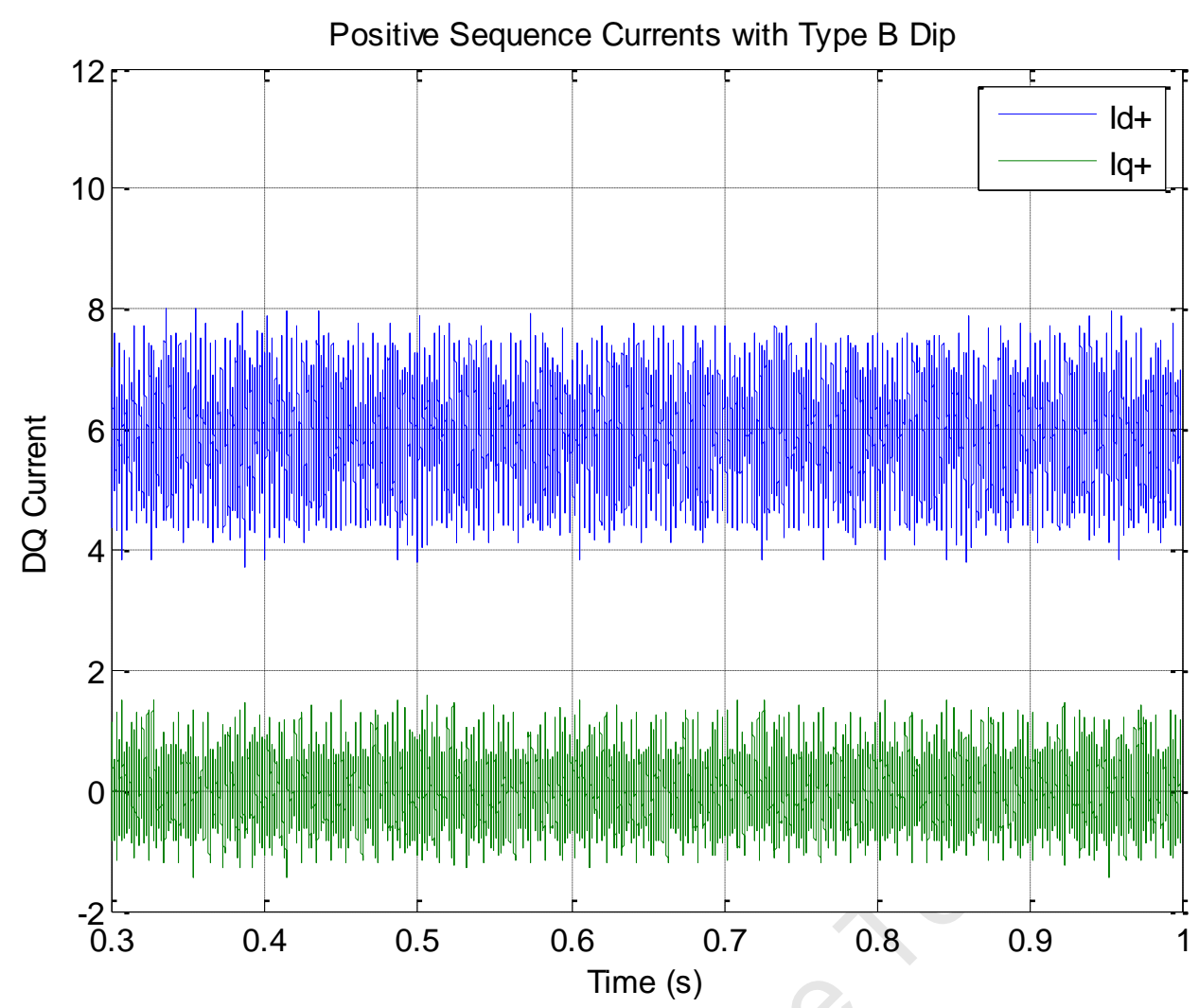

Figure 74:Simulated Dual Vector Control DQ+ Currents with Type B Dip

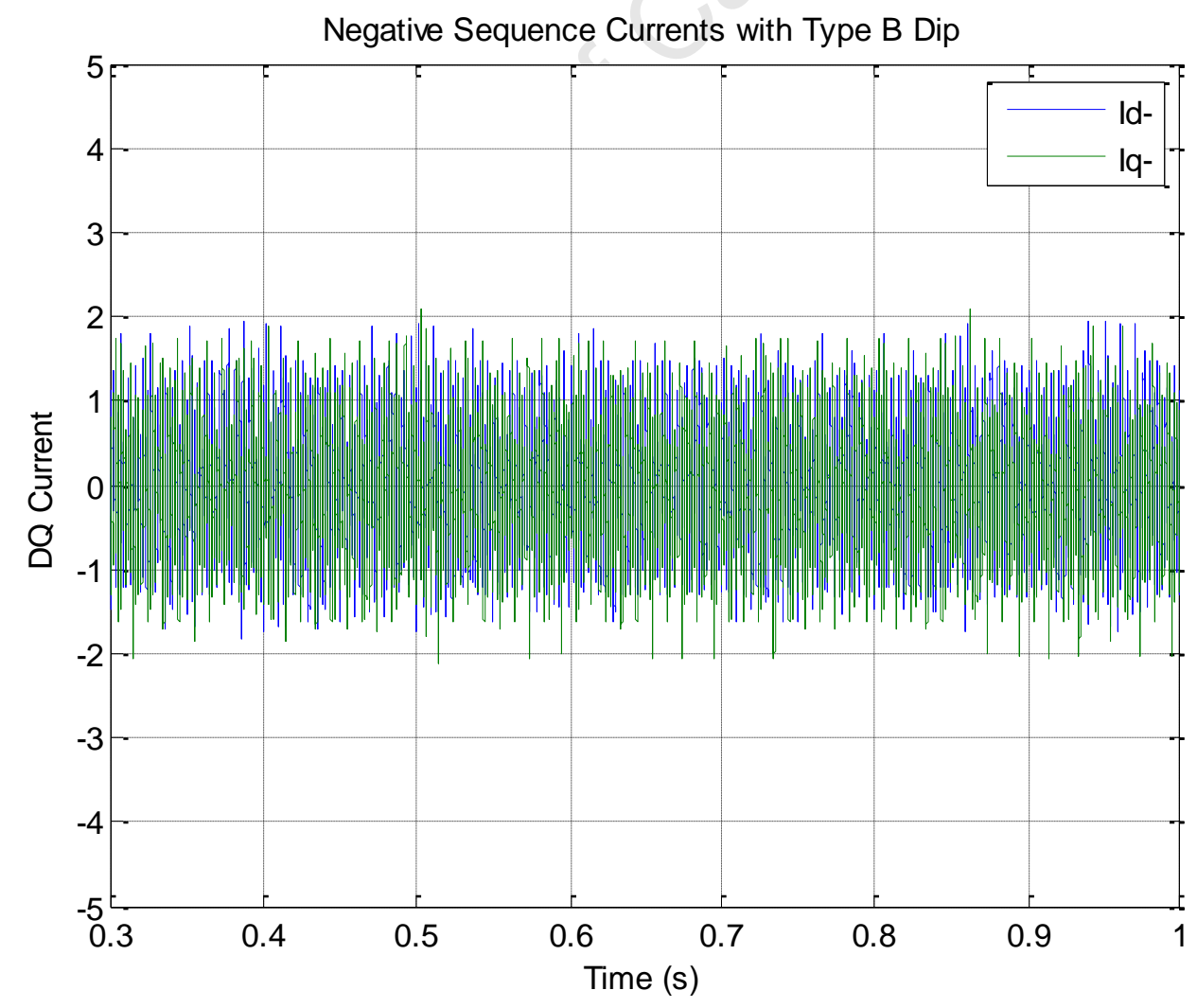

Figure 75:Figure 74:Simulated Dual Vector Control DQ- Currents with Type B Dip 


\subsection{Simulated Machine MPPT Under Turbulent Conditions}

The MPPT capability of the system is tested by simulating the generator side control, including the wind turbulence model and the MPPT algorithm. The overall combination of the grid side, generator side, MPPT, turbine emulator and turbulence model could not be implemented in simulation due to the complexity of the complete system. A simulation of this type would require significant computational power, which was not available for this research. To overcome this limitation, the grid side was assumed to be working correctly and the DC link Voltage was modelled with a DC voltage source.

Figure 76 below shows the wind speed with turbulence included, at a nominal speed of $7 \mathrm{~m} / \mathrm{s}$. The wind speed varies between 6.5 and $8.6 \mathrm{~m} / \mathrm{s}$ during a period of 30 seconds which was the longest period of time the simulation could run for due to computer memory limitations.

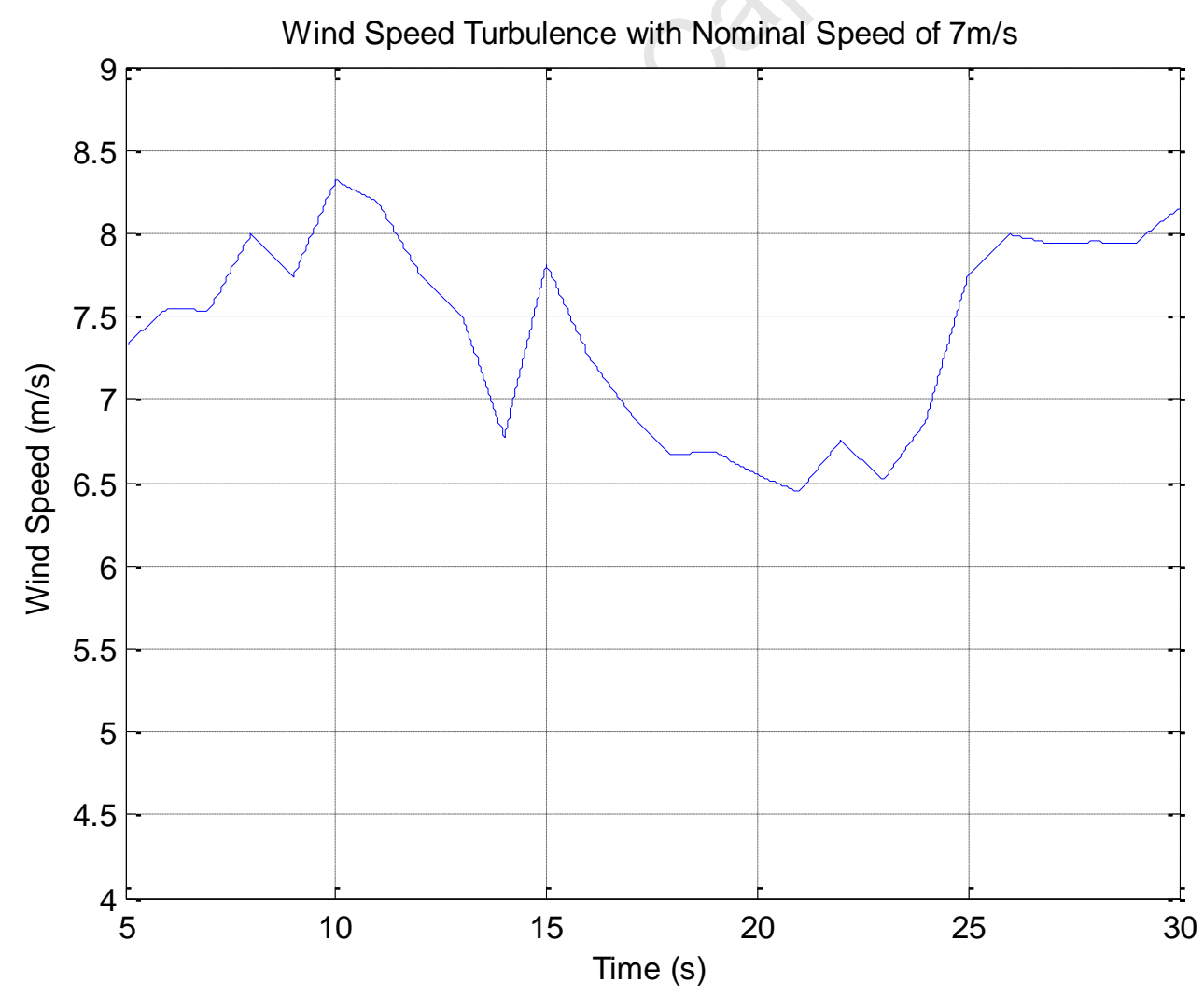

Figure 76: Simulated Wind Speed Turbulence with a Nominal Speed of $7 \mathrm{~m} / \mathrm{s}$ 
Figure 77 below shows the speed tracking capability of the generator by comparing the actual generator speed to the reference speed calculated by the MPPT algorithm. The performance of the speed control is excellent considering the torque disturbances that are introduced by the turbulent conditions. The accuracy of the performance is confirmed by observing the power coefficient over the simulated time frame in Figure 78. The value of $C_{p}$ remains within $0.17 \%$ of its maximum value. The largest deviations in $C_{p}$ are found at the same time as the largest changes in wind speed.

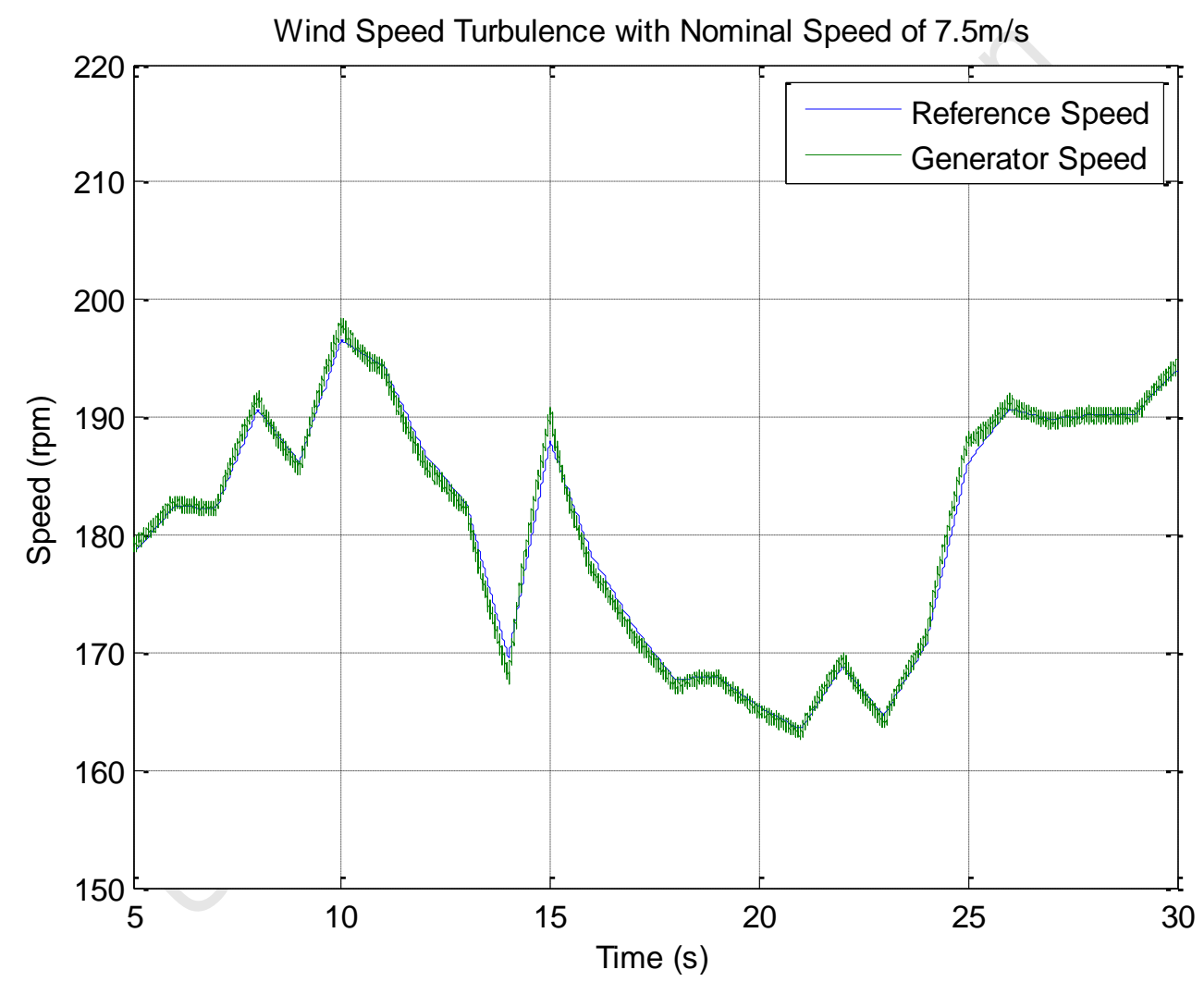

Figure 77: Simulated Comparison Between Actual and Reference PMSG Speed 


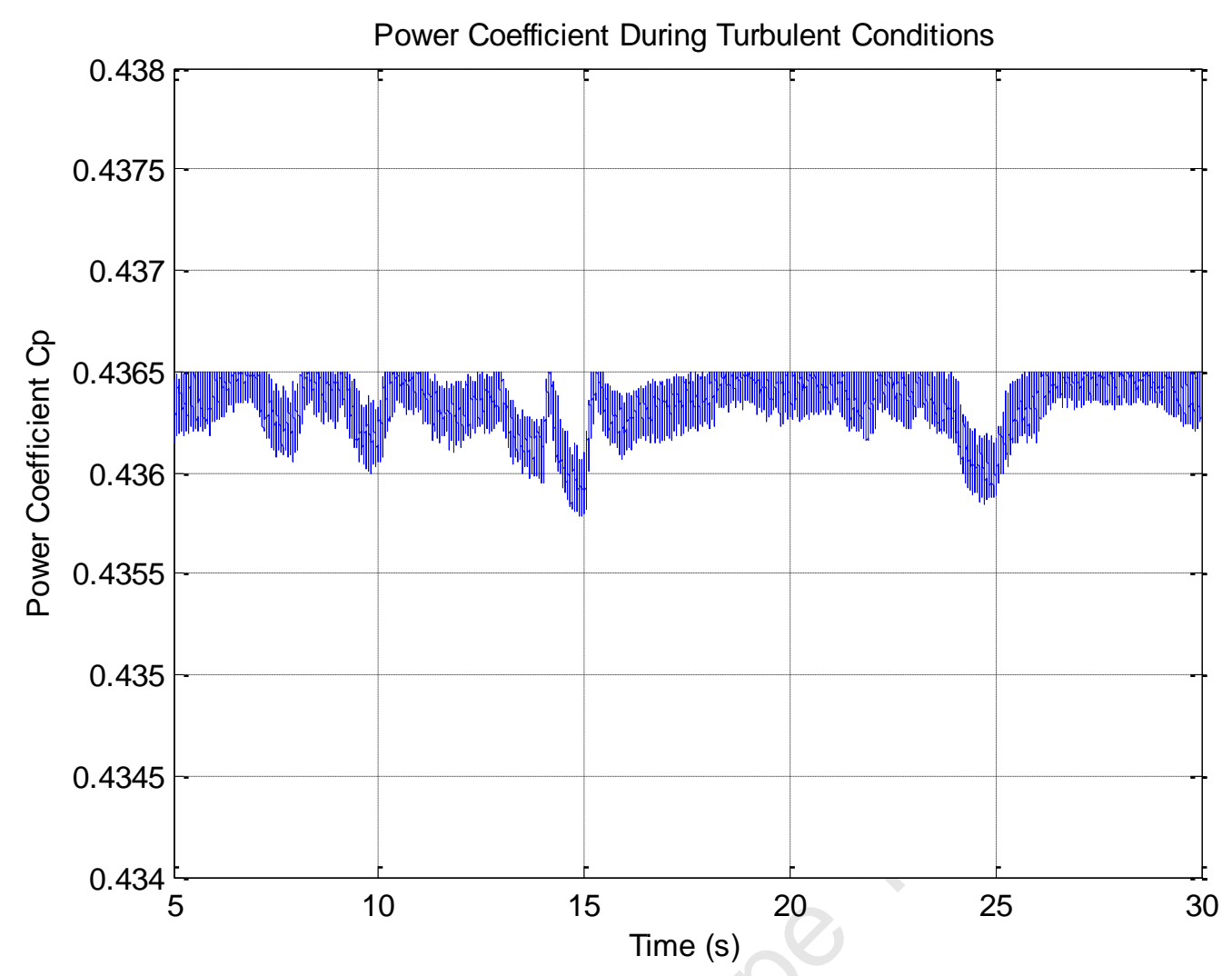

Figure 78: Simulated Cp During Turbulent Conditions

\subsection{Conclusion}

The different components of the system were simulated in this chapter, in order to validate performance before implementing the system experimentally. The simulations included detailed validation of the turbine emulator, generator and grid-side converters, as well as the MPPT algorithm. The simulations of the grid side converter showed that balanced grid currents were achieved under unbalanced supply voltage conditions. It can be concluded that correct operation of the system was achieved in simulation and that experimentation can commence. 


\section{CHAPTER 6}

\section{LABORATORY IMPLEMENTATION}

This chapter introduces the different components of the experimental setup including the hardware and the software that was designed. Part of this thesis was to develop a flexible test rig to upgrade the infrastructure of the machines laboratory which is presented in this chapter. The back-to-back converter and its components are then discussed followed by the measurement and instrumentation equipment. The remainder of the chapter presents the system protection and the technique used to implement unbalanced supply voltage conditions.

\subsection{Flexible Rig}

The test rig was designed to have room for a wide variety of generators. The rig structure accommodate an induction machine, a torque transducer and a generator that may be changed. The base of the structure consists of two I-Beams which are laid out in parallel. The beams have slots machined on one end to allow for different sizes of generators to be added to the system. The I-Beams are illustrated in Figure 79.

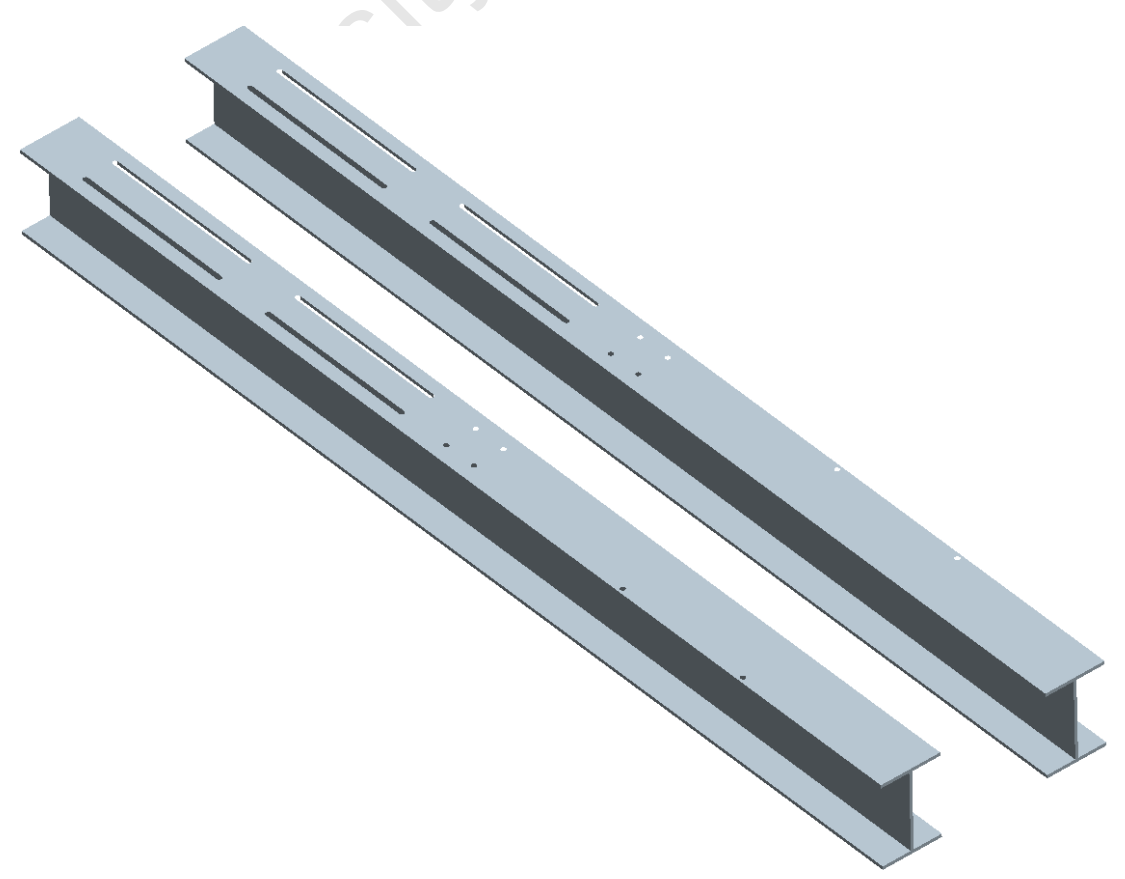

Figure 79: I-Beams With Machined Slots 
The induction machine and torque transducer are mounted at the correct heights using sets of pedestals which are illustrated in Figure 80.

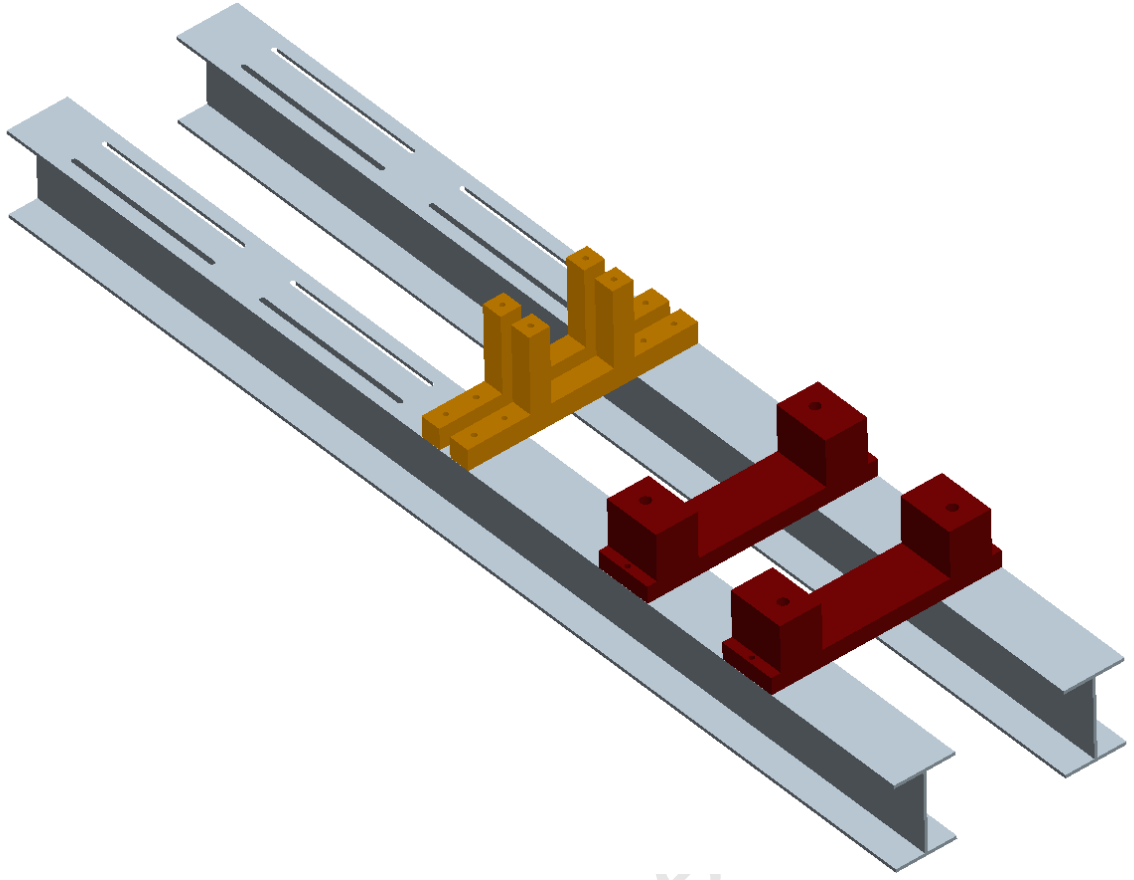

Figure 80: Pedestals Mounted on I-Beams

The generator mount is designed so that the machine may be moved in both the horizontal and vertical axis in order to align the machine. It consists of two cross beams that are laid on top of one another. The upper beam has a pair of bolts sunk into it that fit through the lower beam. The height of the top beam is set by adjusting a set of nuts on the bolts as indicated in Figure 81.

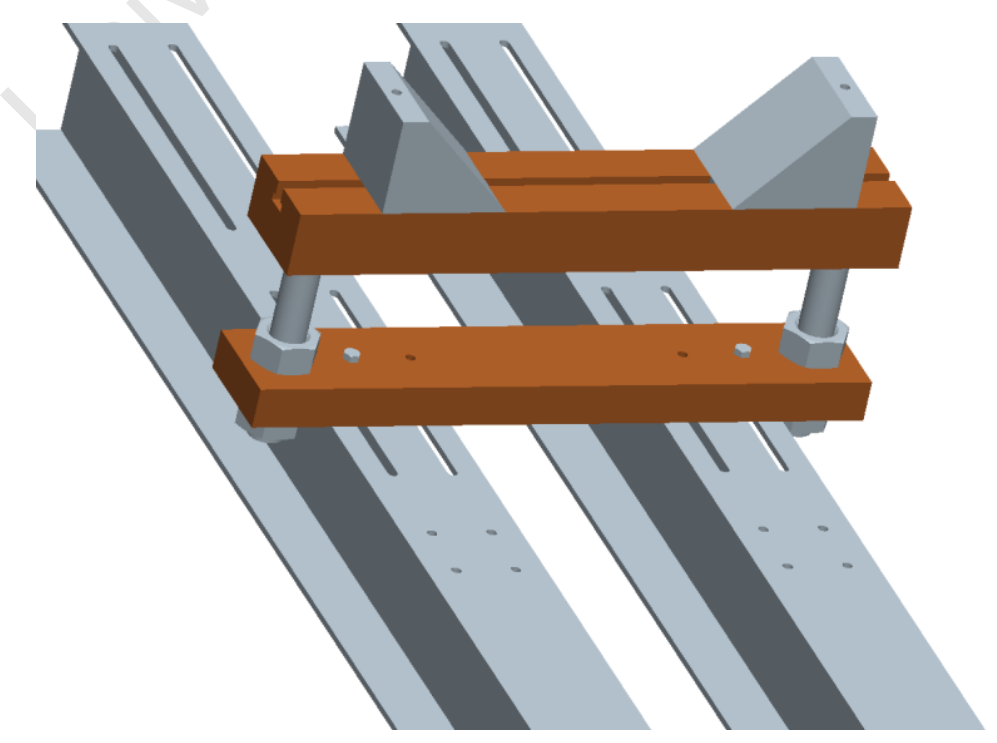

Figure 81: PMSG Positioning System 
The generator is laid on two wedges which can slide in a horizontal slot that has been machined out of the upper beam. The wedges are moved in the slot to position generator horizontally. A third beam with another set of horizontally adjustable wedges is placed on top of the generator in order to clamp it down. For additional holding force, four high tension stainless steel rods are positioned between the two top beams and are tightened as illustrated in Figure 82.

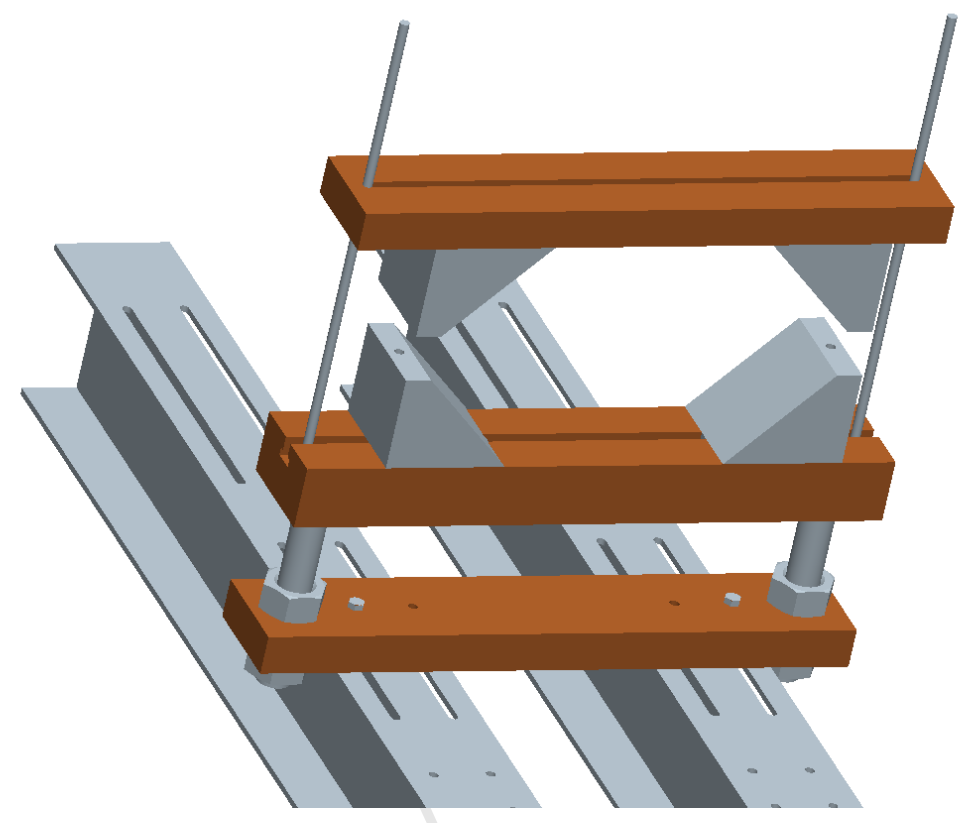

Figure 82: PMSG Clamping System

When the generator is operating at full power there is a large torque acting on the machine body and an equivalent force needs to be applied to keep it in place. The generator is clamped in place at four points but the frictional coefficient between two metals is not substantial. Anti-vibration rubber mats are added to the face of the wedges in order to increase the friction that can be produced from the force. The generator mounting is displayed in Figure 83 and the entire rig is displayed in Figure 84. 


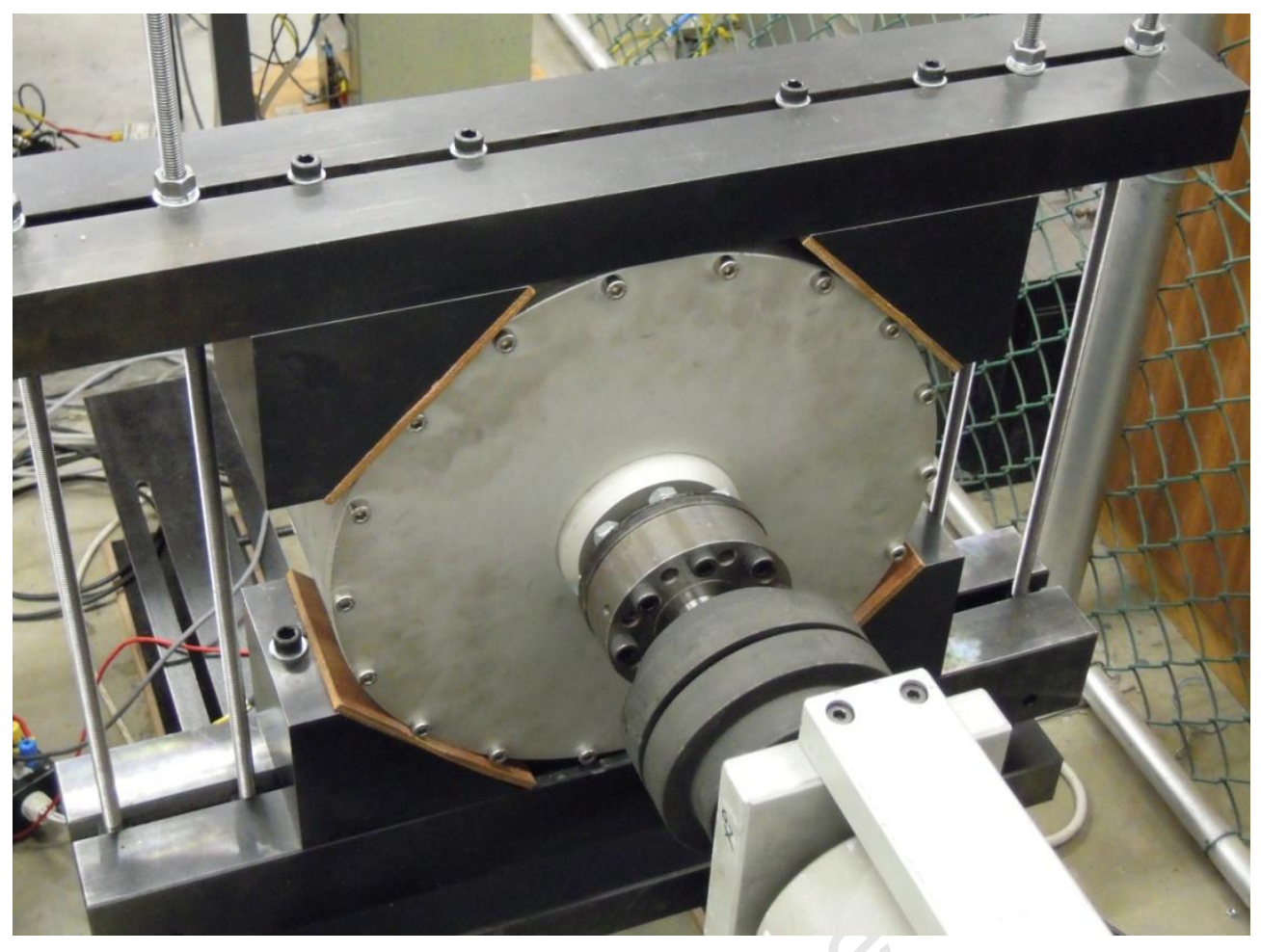

Figure 83: PMSG Mounted on Test Rig

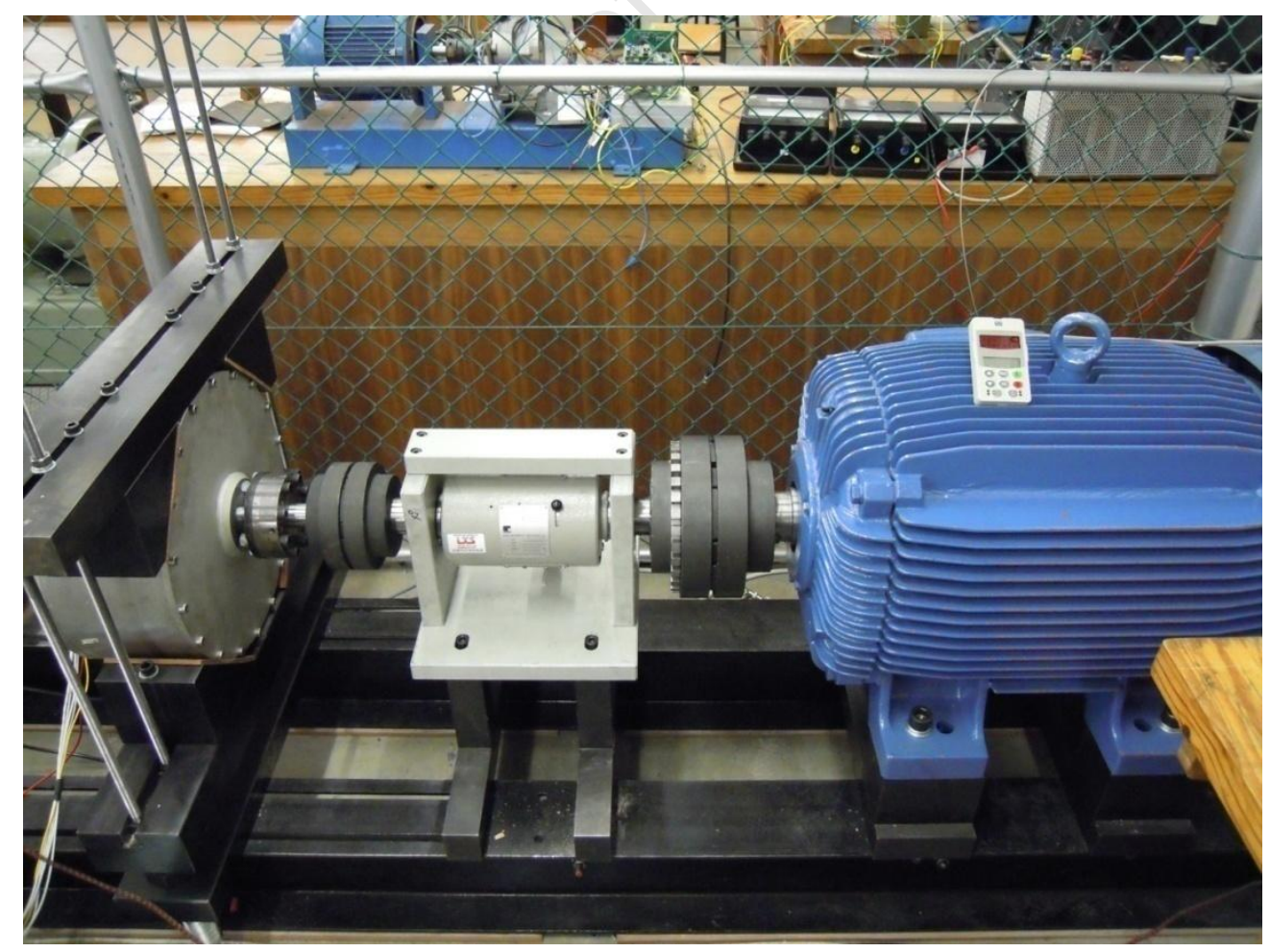

Figure 84: Fully Assembled Test Rig 


\subsection{Back-to-Back Converter}

The WECS Converter is a Semikron back-to-back full bridge converter. It has a built in DC Link with a capacitance of $4700 \mu \mathrm{H}$. The switches are IGBT's and each half-bridge has its own driver which receives its signals from the DSpace kits. One converter is used to control the generator side whilst the other controls the grid side. A photo is shown in Figure 85.

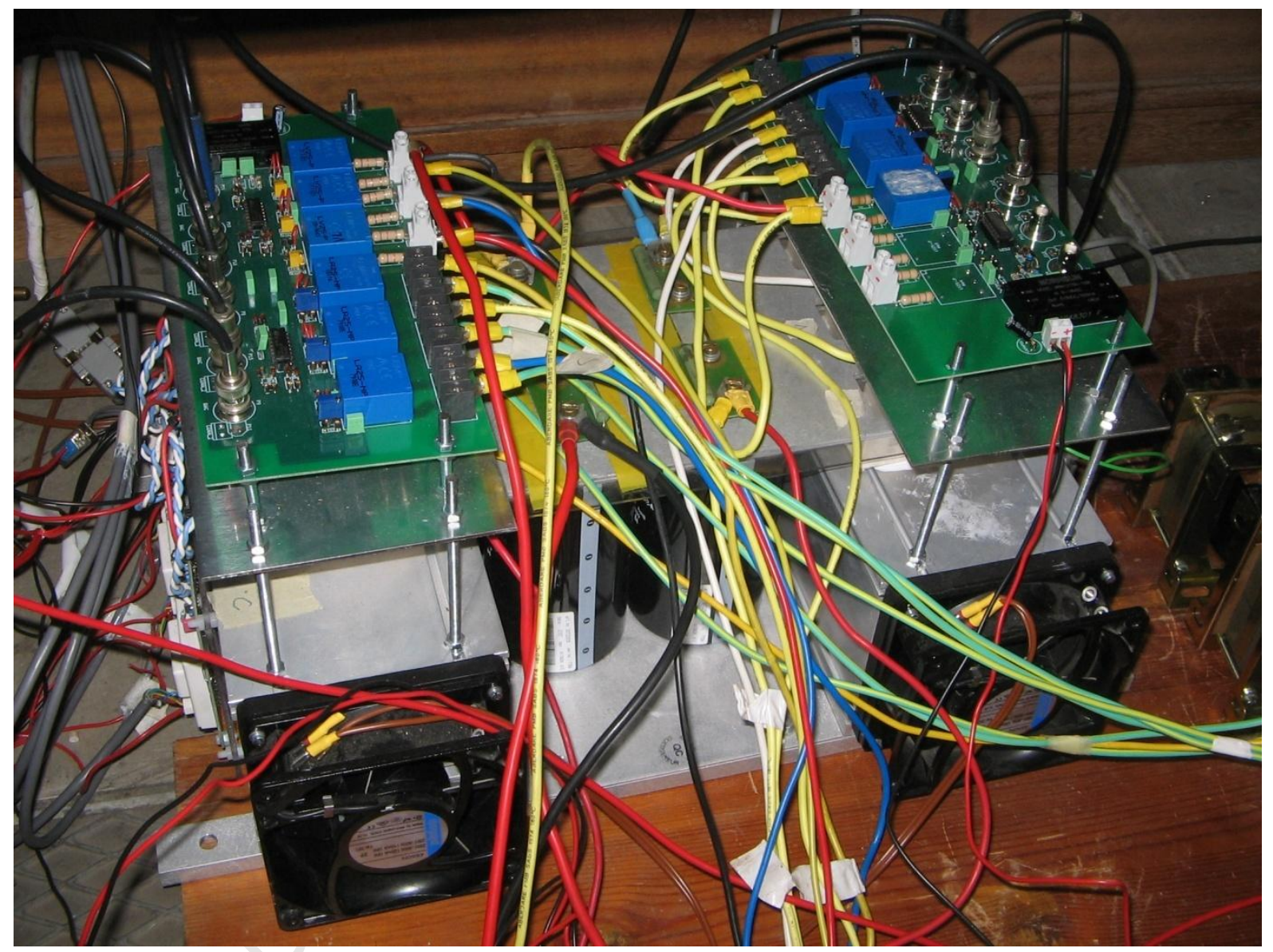

Figure 85: Back-to-Back Converter 


\subsubsection{IGBTS}

Semikron SKM100GB128D IGBT's are used as switches for the converters. They have a maximum voltage rating of $1200 \mathrm{~V}$ and a maximum current rating of $100 \mathrm{~A}$ enabling them to handle the maximum ratings of the system. The maximum repetitive current rating at a period of $1 \mathrm{~ms}$ is the $150 \mathrm{~A}$ which is used along with Figure 86 to determine the components used for $V_{C E}$ monitoring that is described further in section 6.2 .2 below.

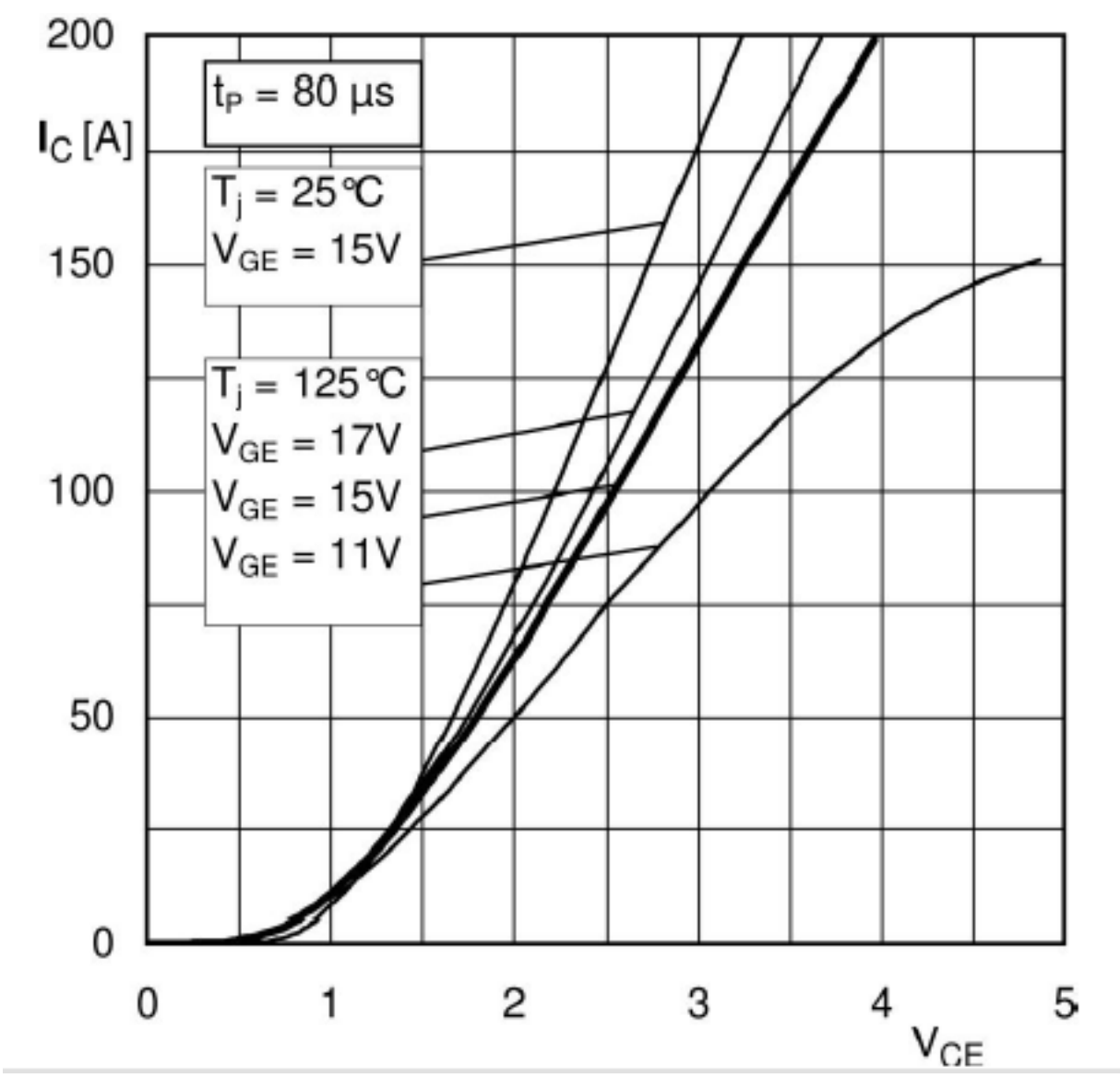

Figure 86: Collector Current vs. Collector-Emitter Voltage for IGBT

A $V_{C E}$ value of 2.75 corresponds to a collector current of $150 \mathrm{~A}$ at an operating temperature of 25 degrees Celsius. Figure 87 shows one of the IGBT half bridges. 


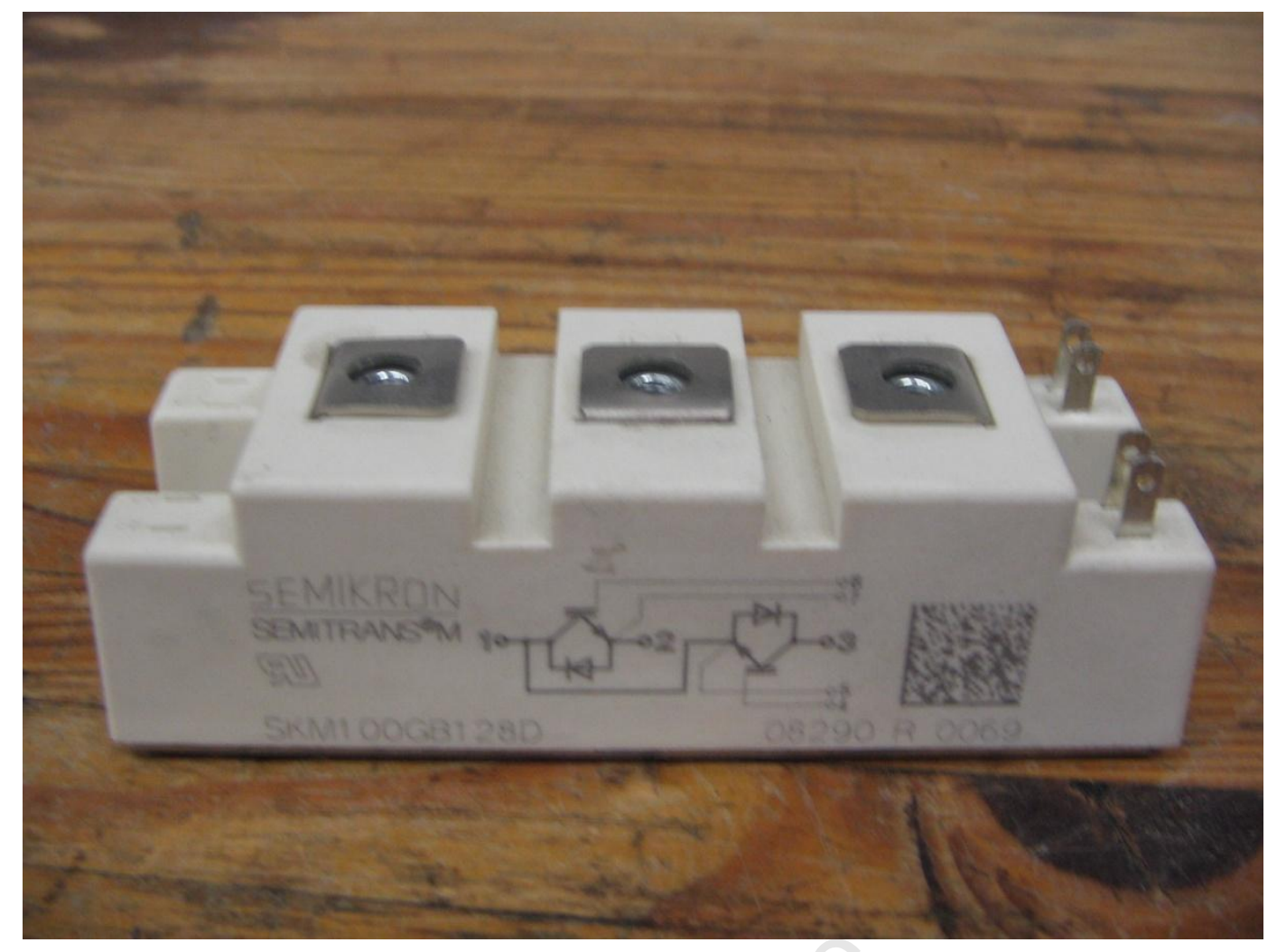

Figure 87: Semikron IGBT Half Bridge

\subsubsection{Drivers}

The drivers for the IGBT's are Semikron SKHI22BR. The high power side is isolated from the logic side by transformers for DC voltages up to $1200 \mathrm{~V}$ which is sufficient for this project. Each driver features output signals for the high and low switches of a half bridge with a built-in interlock time to prevent shorting the switches. The drivers also monitors the collector-emitter voltage $V_{C E}$ to detect current spikes. If an error is detected by the interlock or collector-emitter voltage monitors, the driver will shut down immediately and produce an error signal.

The $V_{C E}$ monitoring reference is set by choosing a suitable resistor $R_{C E}$ and the delay before the monitoring is activated, is set with parallel capacitor $C_{C E}$. Equations $6.1,6.2$ and 6.3 are used to determine these values.

$$
V_{C E s t a t}(V)=\frac{10 R_{C E}(k \Omega)}{10+R_{C E}(k \Omega)}-1.4
$$




$$
\begin{gathered}
t_{\min }=\tau_{C E} \ln \left[\frac{15-V_{\text {CEstat }}(V)}{10-V_{\text {CEstat }}(V)}\right] \\
\tau_{C E}(\mu s)=C_{C E}(n F) \frac{10 R_{C E}(k \Omega)}{10+R_{C E}(k \Omega)}-1.4
\end{gathered}
$$

The error signal is $15 \mathrm{~V}$ under normal operating conditions and switches to $0 \mathrm{~V}$ when a fault is detected. The error signals from the drivers are passed through a circuit to comply with the TTL protocol of the DSpace digital 10 module which is further discussed in section 6.3.3. Figure 88 shows the drivers mounted on the converter stack.

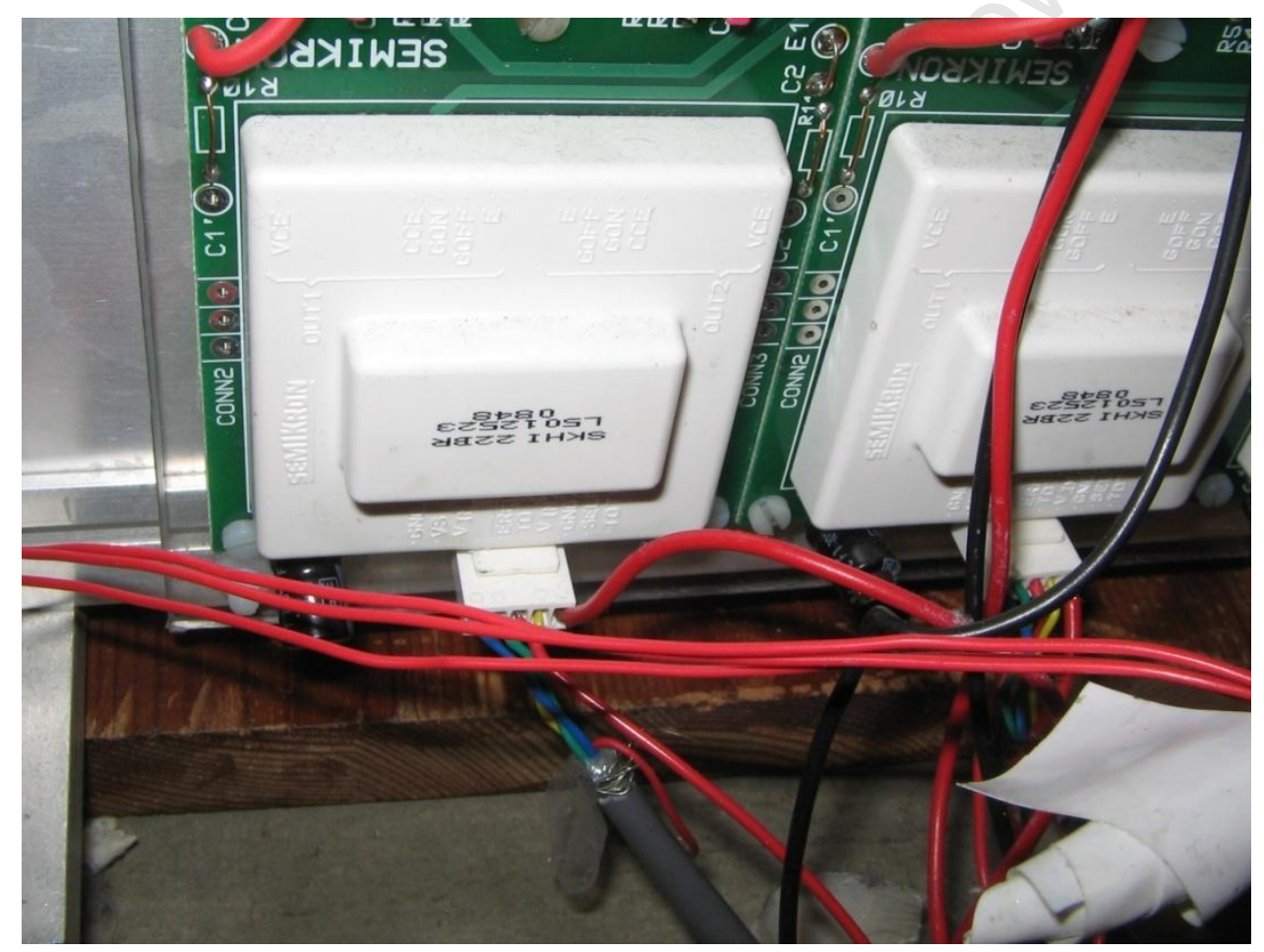

Figure 88: Semikron Driver Mounted on Converter

\subsection{Instrumentation and Measurement}

This section discusses how all of the system measurements are implemented as well as how the measurements are interfaced with the control system. 


\subsubsection{Current and Voltage Measurement}

LEM Modules are used to measure the current and voltages of the entire system. Six current sensors in total are used, three for the grid side and three for the gen side. Four voltage sensors are used, three for the grid side and one for the DC link voltage. The sensors are mounted on custom built boards which are mounted above the converter. The circuit diagrams for the boards can be found in Appendix B.

The current LEM modules have an attenuation ratio 1000:1 between the primary and secondary side currents and are rated for $25 \mathrm{~A}$. The secondary current is passed through an output resistor to create the desired output voltage. The maximum rated current of the system is $9.09 \mathrm{Arms}$ which has a peak value of $12.85 \mathrm{~A}$. The maximum input voltage of the DSpace analogue-to-digital converter is $10 \mathrm{~V}$. A safety factor of 2 is used when deciding on the output resistor value. The resistor value is chosen so that an instantaneous primary current of $25 \mathrm{~A}$ will produce an output voltage of $10 \mathrm{~V}$.

The Voltage LEM modules have a primary to secondary current ratio 1:2.5 and a maximum input voltage of $500 \mathrm{~V}$. The DC Bus operates at $650 \mathrm{~V}$ so a resistor is placed on each of the input lines, forming a voltage divider. The output resister is chosen so that an input voltage of $1000 \mathrm{~V}$ will produce an output of $10 \mathrm{~V}$ incorporating a safety value of 1.54 .

Figure 89 and Figure 90 illustrate the operation of the current and voltage LEM modules while Figure 91 shows a completed mounting board.

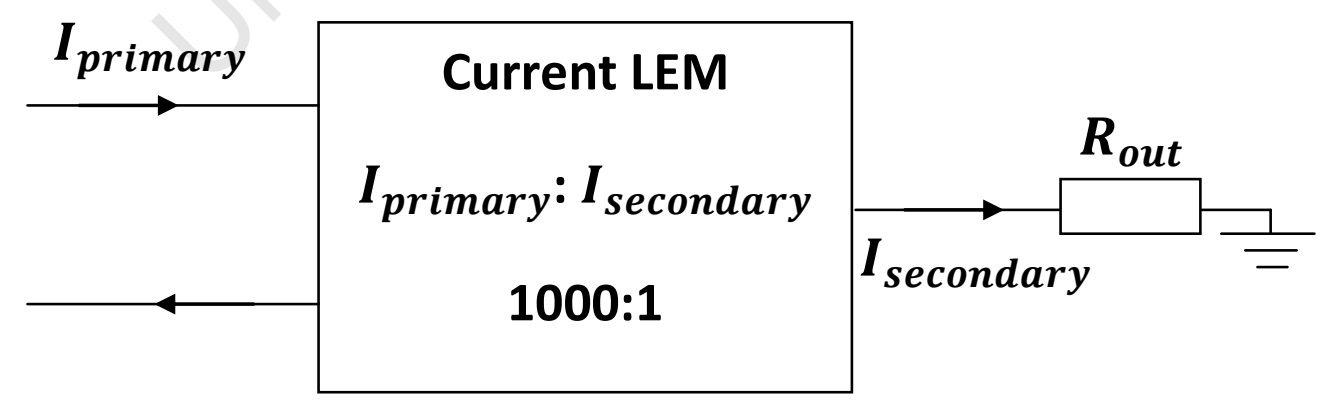

Figure 89: Operation of Current LEM 


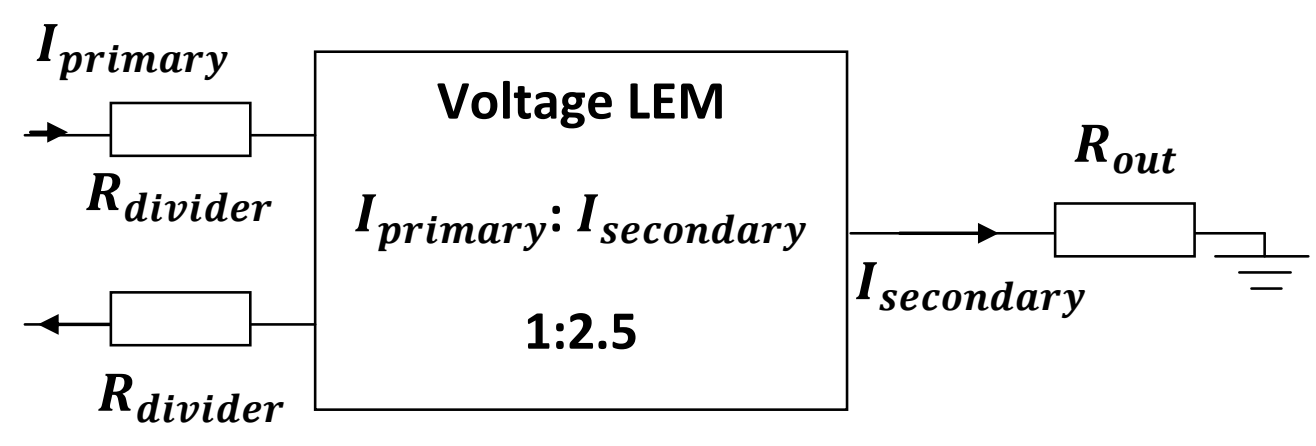

Figure 90: Operation of Voltage LEM

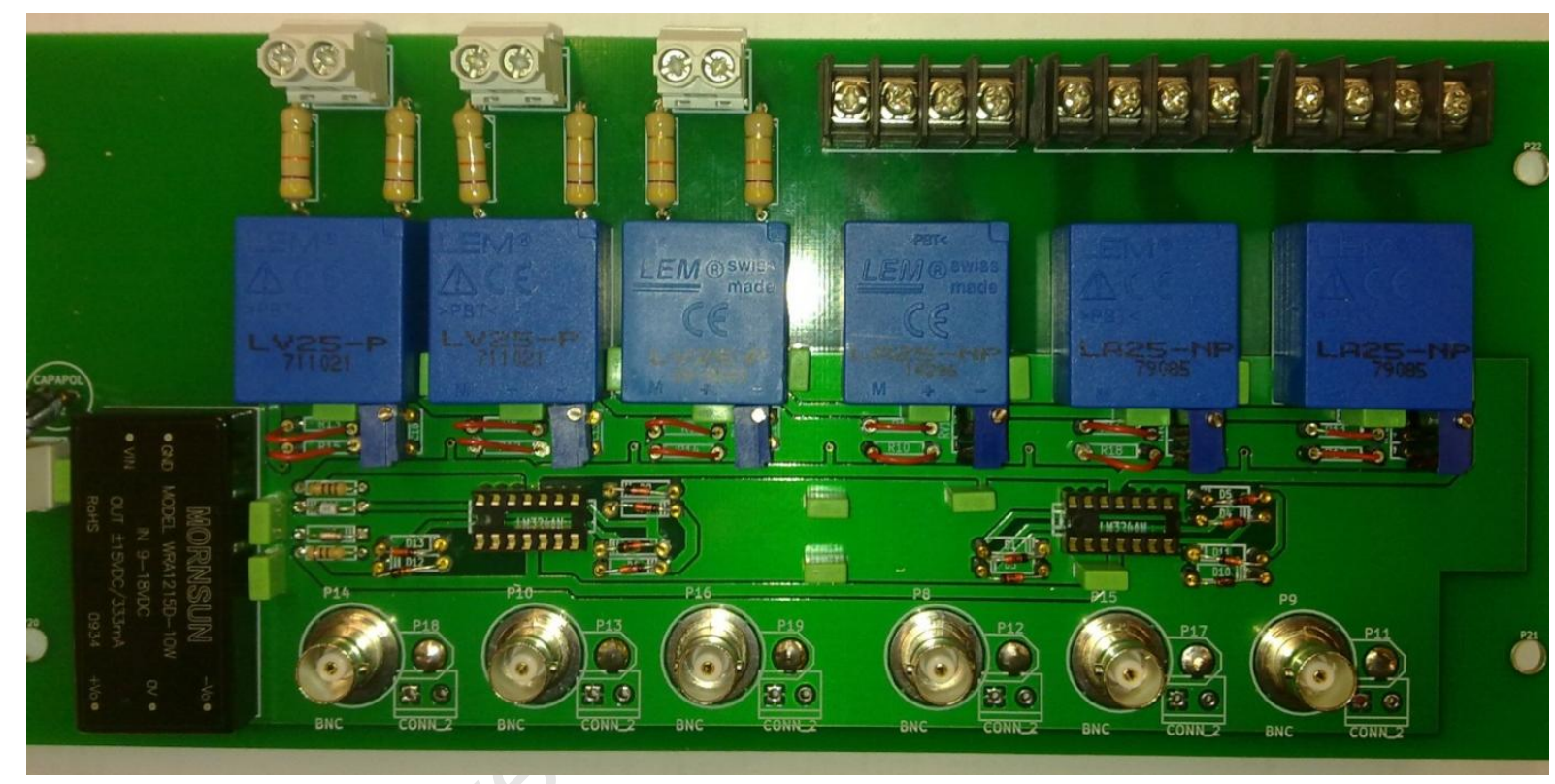

Figure 91: LEM Sensors Mounted on PCB

\subsubsection{Encoder Position and Speed}

A KÜBLER Type Sendix incremental Type 5000 encoder is attached to the shaft of the generator in order to calculate the instantaneous speed and position. The encoder has 5000 points per revolution including an index point. The speed and positions are determined as follows. The index pulse is aligned with the $d$-axis by following procedure 1 in appendix $A$. As the generator rotates, the pulses are counted until the next index pulse is detected and the counter is reset. The position of the rotor can be determined by equation 6.4 .

$$
\theta=\frac{2 \pi}{5000} \text { count }
$$




$$
\omega=\frac{\theta_{2}-\theta_{1}}{t}
$$

$$
t=\mathrm{nT}
$$

Where count is the current value of the incremental counter. The speed $\omega$ is determined by equation 6.5 where $\theta_{1}$ and $\theta_{2}$ are the rotor position at a set number of samples apart. $t$ is the time over which the speed calculation is made where $\mathrm{n}$ is the number of samples and $\mathrm{T}$ is the sampling time. If an overflow occurs, i.e. the index is passed before the second value is sampled, and the second value is lower than the first value and the calculation is discarded. Once the speed has been determined, it is averaged over the last ten speed calculations in order to smooth out the results in case of any miscalculations. The delay caused by this method of determining the speed is difficult to model because it is difficult to predict the number of calculations that are going to be thrown out. The faster the machine rotates, the greater the number will be therefore making the delay some function of the speed. The averaging of the speed will also create a delay under transient conditions. An acceleration will cause the calculated speed to be less than the instantaneous speed whilst a deceleration will cause the calculated speed to be higher than the instantaneous speed. When the machine is under steady-state conditions, the averaged speed will be equal to the instantaneous speed. Figure 92 shows the encoder mounted on the generator.

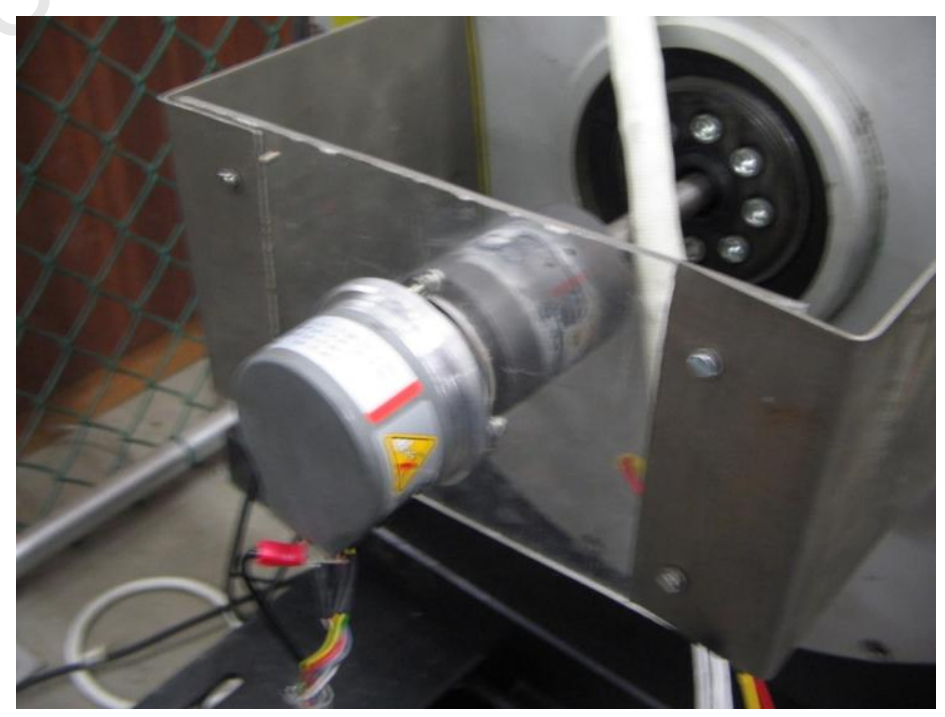

Figure 92: Encoder Mounted on PMSG 


\subsubsection{Error Signals and Relays}

All error signals are passed through a circuit which adapts the signals to TTL format. The signals are sent through a voltage divider which regulates the voltages to the correct $0-5 \mathrm{~V}$ level. The resulting TTL level signals are then passed through a unity gain operational amplifier buffer circuit which has its outputs clamped with diodes in order to prevent large voltages being sent to the digital 10 in the case of circuit malfunction. The contactors are controlled by relays which receive their signals from the DSpace kit digital IO but are formatted through another circuit which adapts the signals to the required 0-24V signals. Both of the formatting circuits described above are built onto a single board which is illustrated in Figure 93. The circuit diagram can be found in appendix B.

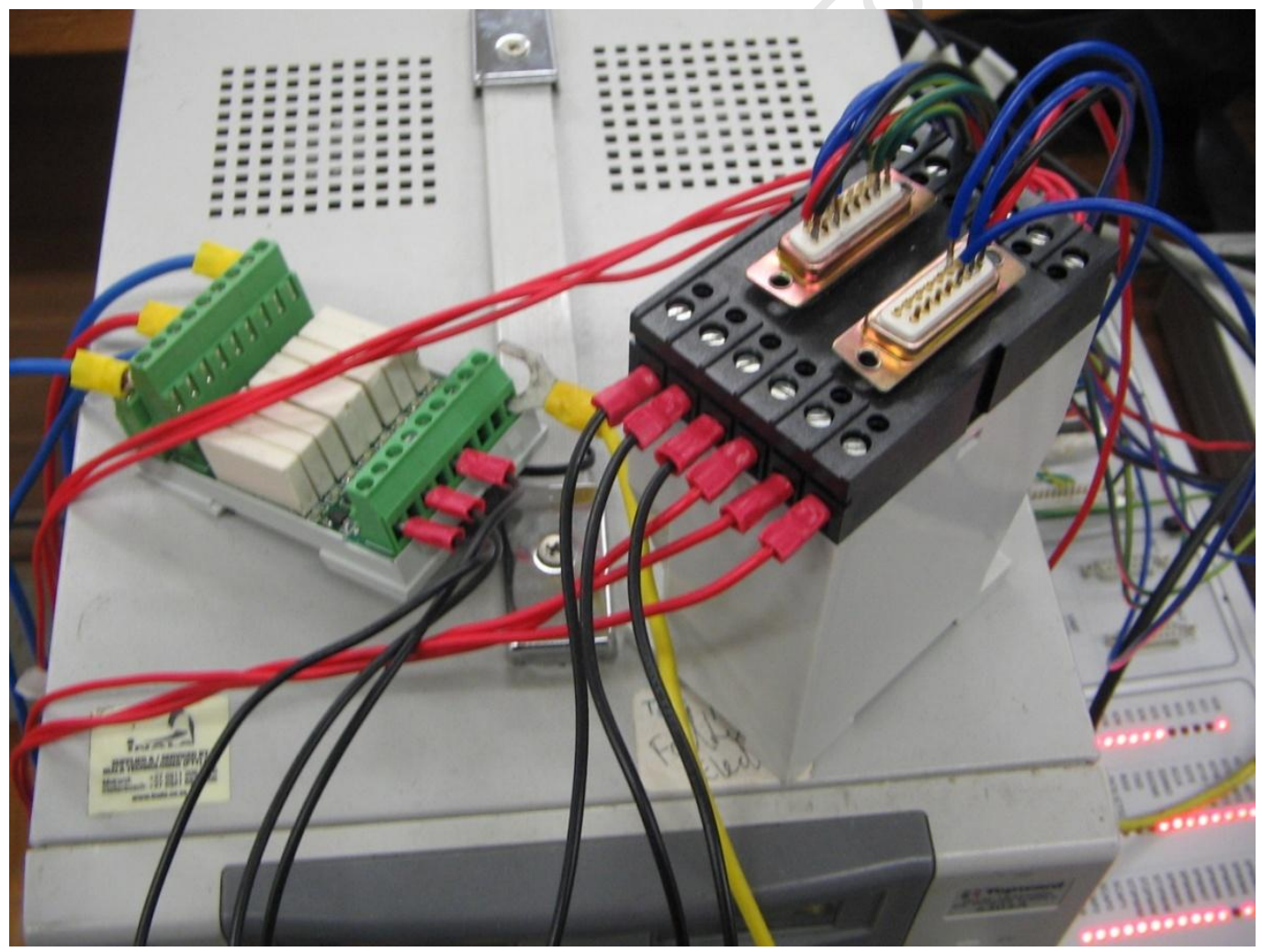

Figure 93: Error and Relay Control Circuits 


\subsection{Protection}

Protection is a vital part of any system in order to prevent component failure as well as to prevent hazardous working conditions. This section discusses the protection techniques that are implemented in the hardware and software of the system. A full protection diagram is presented in Figure 94.

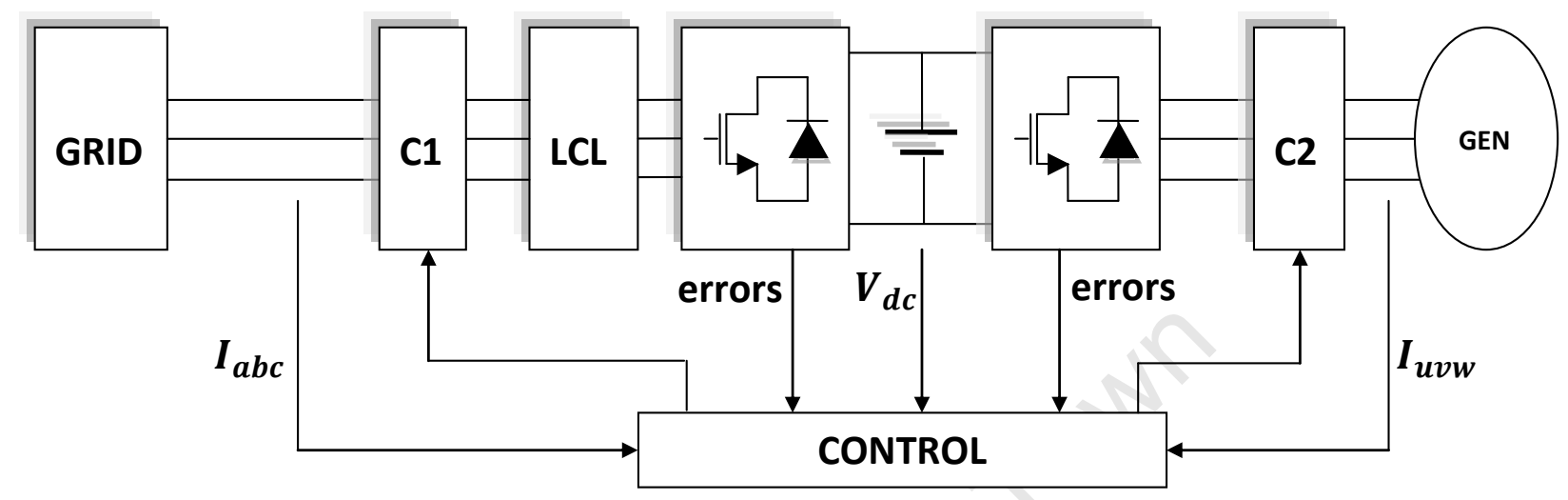

Figure 94: Protection Control Block Diagram

\subsubsection{Hardware}

System safety is ensured by contactors that are controlled by the system control. A contactor provides a connection between the grid and the grid side converter after the grid filter. Another contactor is located between the generator and the converter. The contactors are linked to the converter error signals as well as the DC Link voltage. If any of the converter error signals are generated or if the DC Link voltage rises too high, both contactors will switch out shutting down the system. The driver signals will also be turned off to stop the switching.

\subsubsection{Software}

Protection is built into the software in the form of current limits and signal saturation. The $d q$ currents are monitored and a limit is set for each. If this limit is reached, the contactors are switched off as well as the driver switching signals. Signal saturation is used to limit the voltage signals that can be produced by the converter, thereby limiting the maximum current that can occur. 


\subsection{DSpace}

DSpace 1104 kits are used to interface between the software and the system hardware. A major advantage of using the DSpace kit is that it minimises development time drastically. The kit is able to have MATLAB Simulink models loaded directly onto it, greatly simplifying the process of changing over from simulation to experimental implementation. DSpace provides a Simulink toolbox so that the simulation code can be adapted to the implementation code without major changes. The toolbox includes a wide range of premade block providing easy access to the PWM, DAC, ADC and digital IO features of the kit. Figure 95 shows the DSpace 1104 kit.

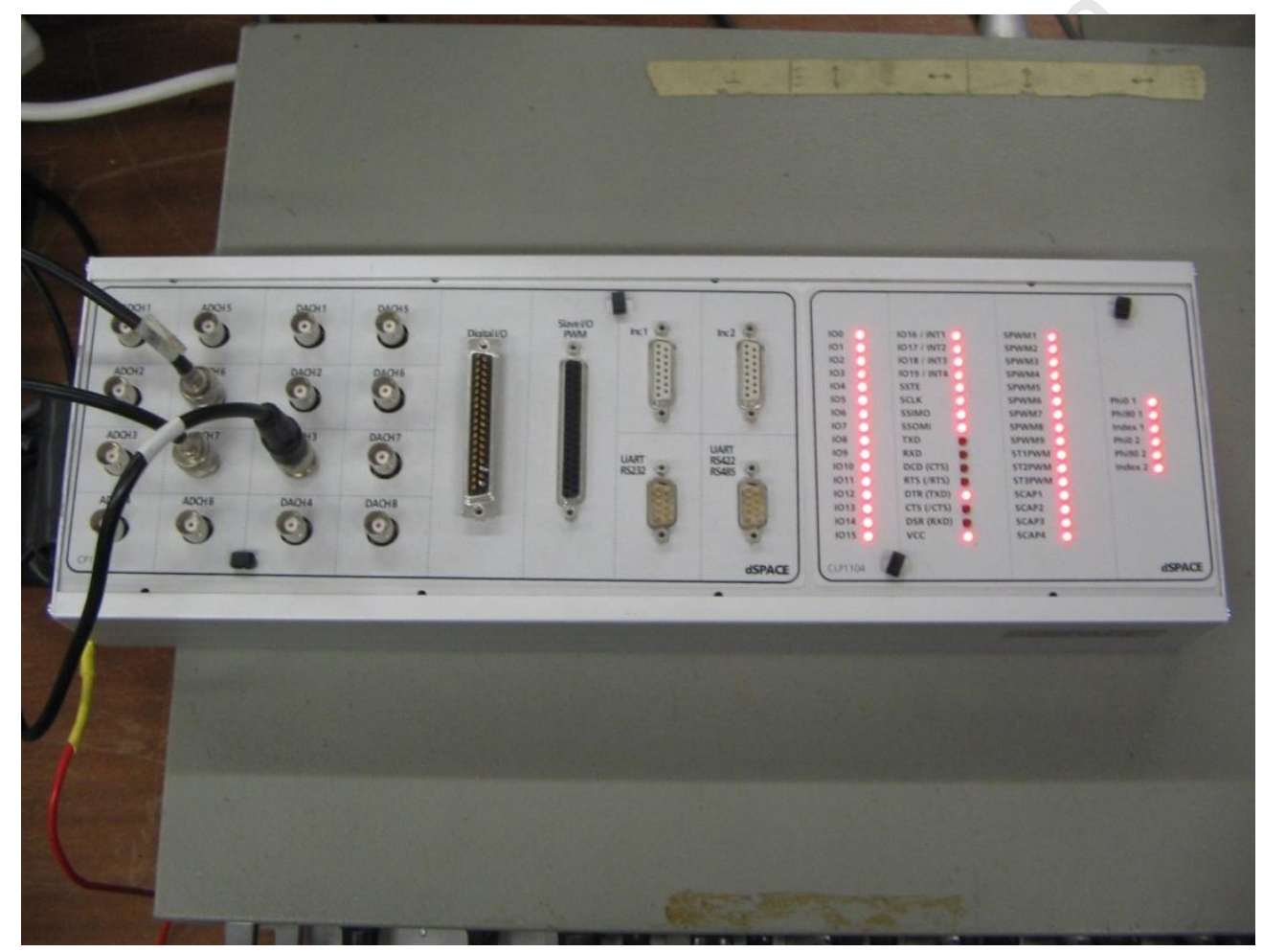

Figure 95: DSpace 1104 Kit

Although the kits are very user friendly as described above, there are two major disadvantages when using the 1104 DSpace kits. The kit has limited processing power with an onboard $250 \mathrm{MHz}$ Motorola 64 bit floating point processor which leads to a fairly large computational time. This effects the sampling time directly which in turn limits the allowable complexity of the system. The input and output connectors of the kit are not isolated but are instead referenced to earth. Any noise on the earth plane could then be superimposed on the control signals of the system which in turn can lead to instability and 
unpredictable behaviour. This is especially true for the sensitive grid side control where the slightest change in current can have a large effect on the DC Link voltage. The noise that is picked up from the earth plane is transferred to the voltage measurement which can lead to feedback errors of up to $10 \mathrm{~V}$. The control then tries to compensate for the noise which leads the control to hit its upper and lower saturation limits. The reference currents are then forced in opposite directions, which causes instability resulting in the controller gains needing to be desensitised.

\subsection{Turbine Emulator}

The equipment that is required to produce the desired turbine emulation effects is discussed in this section as well as the selection requirements that need to be met.

\subsubsection{Sizing of Induction Machine}

The induction machine was selected based on the requirements of the generator. The generator is a $6 \mathrm{KW}, 240 \mathrm{rpm}$ machine that can produce a maximum torque of $239 \mathrm{Nm}$. The induction machine needs to be able to meet the torque and speed requirements of the generator. A $30 \mathrm{~kW}, 8$ pole machine was chosen with a rated speed of 750rpm. It is de-rated to a maximum speed of $240 \mathrm{rpm}$ where in the worst case scenario the induction machine can supply $344 \mathrm{Nm}$ of torque. This allows the turbine emulator and the generator to be connected directly without the use of a gearbox and supply the required power for the generator to operate at full power.

\subsubsection{VSD}

The VSD was selected to complement the induction machine as well as control the speed and torque of the machine. A 30kW CFW-09 $380 \mathrm{~V}$ WEG drive with a rated current of $60 \mathrm{~A}$ was chosen. It features sensorless vector control down to speeds of $5 \mathrm{~Hz}$ as well as vector control with an encoder down to zero speed. It also allows external torque and speed commands to be sent to it through its analogue inputs. Figure 96 shows the VSD mounted in its final position next to the recommended fuse box. 


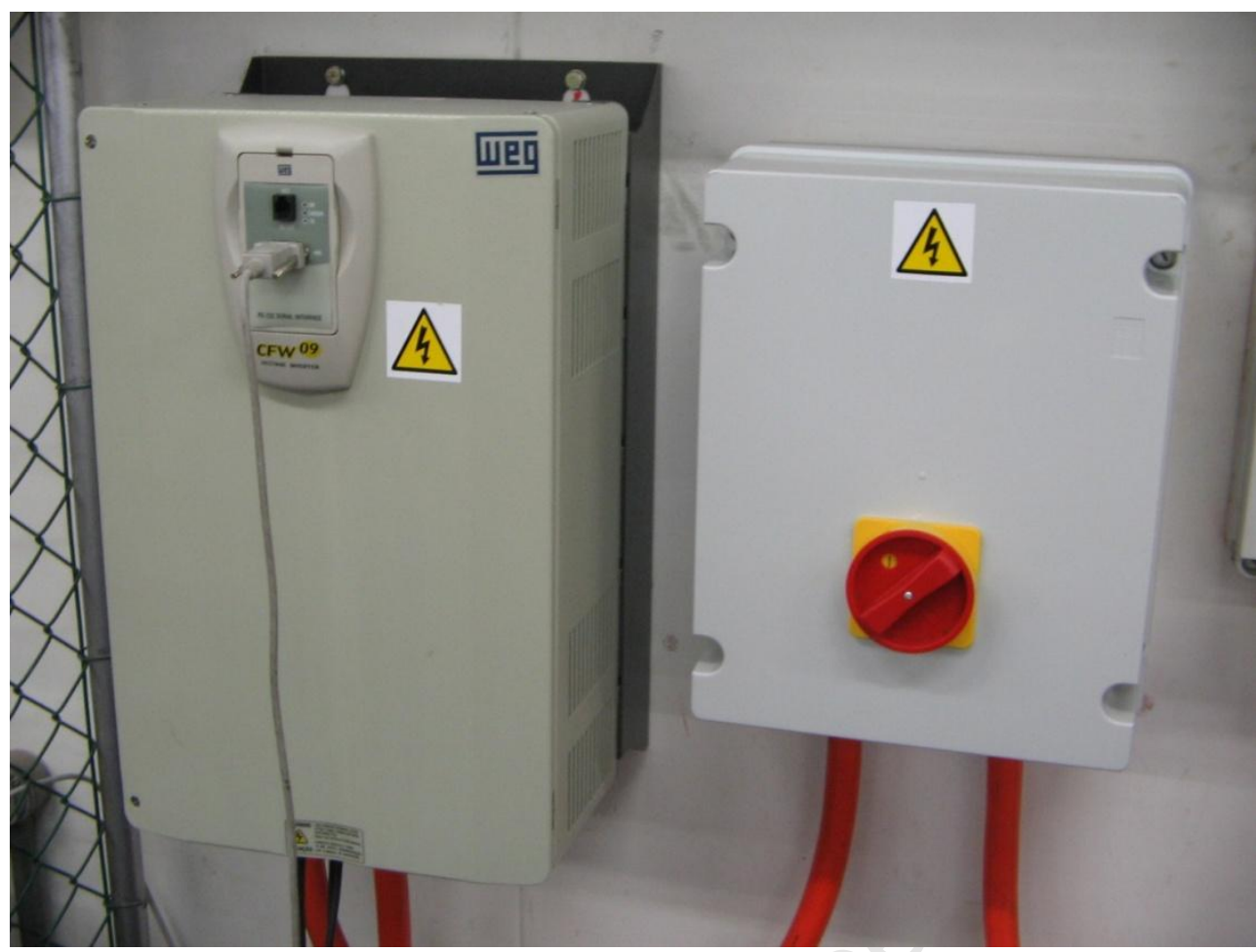

Figure 96: VSD Mounted on Wall

\subsubsection{Control}

The induction machine is controlled using torque control. The reference torque that is calculated from the wind turbine model is sent to the VSD from a DAC port on the DSpace kit. The reference torque sets the maximum forward and reverse torque that can be exerted by the induction machine as a percentage of the maximum machine torque. Another reference signal is sent in the same manner to set the speed reference. This reference limits the maximum speed of the machine which prevents the induction machine from overloading the generator. The machine will try to accelerate to the speed reference applying the torque required to get there up to the maximum allowable torque set by the first reference signal.

\subsection{Implementation of Power Quality Effects}

A series of two variable transformers is used two simulate the required grid situations. The first transformer is balanced and is used to set the starting balanced grid voltages. The second transformer is modified so that each phase can be manipulated separately. In this way, the second transformer is used to simulate dips and swells on any of the phases when required. This method does not allow for short voltage dips in magnitude of less than a few 
seconds because the phases have to be operated mechanically by hand. Figure 97 illustrates the concept in the form of a block diagram whilst Figure 98 displays the transformer setup in the laboratory.

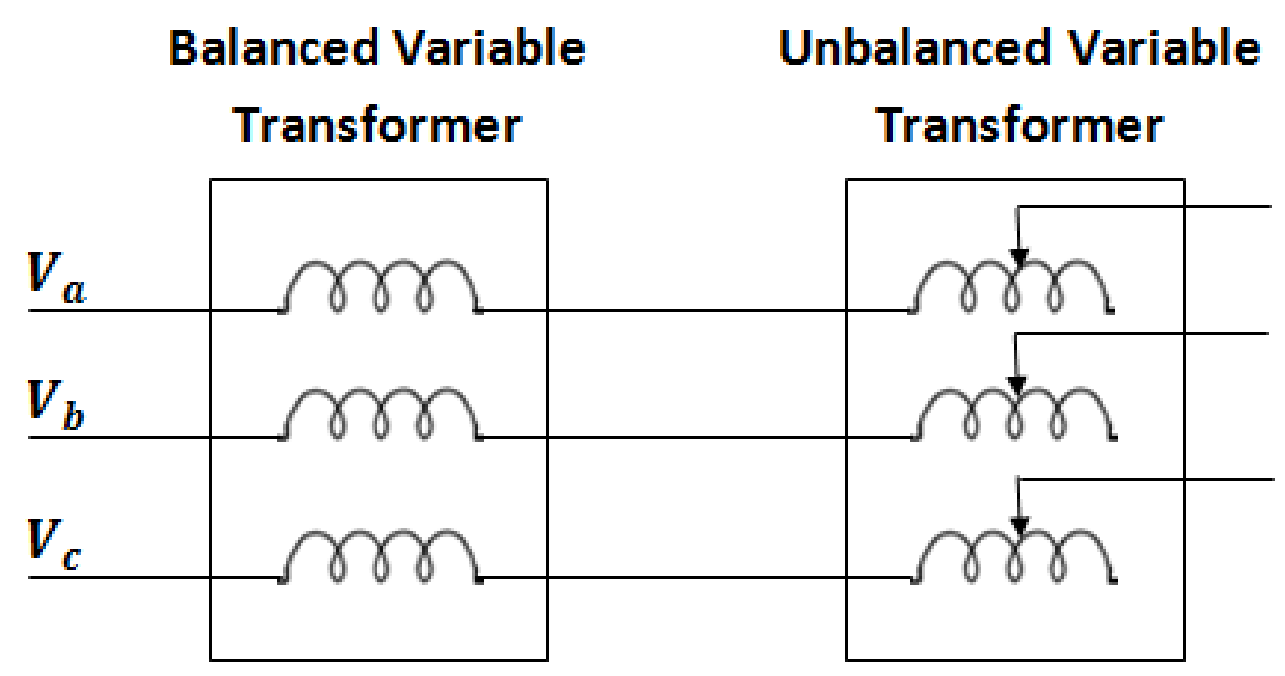

Figure 97: Dip Simulation Setup Block Diagram

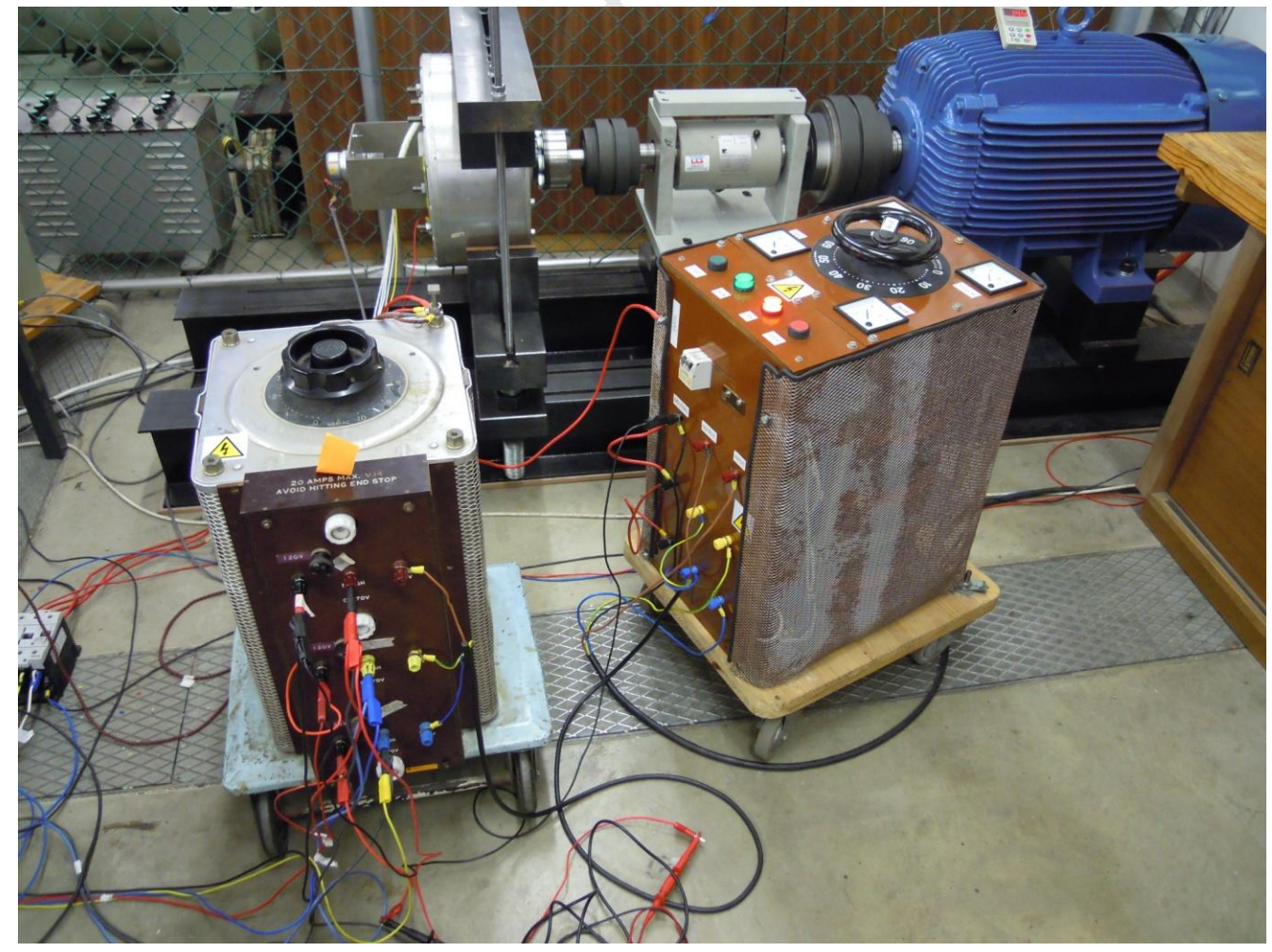

Figure 98: Dip Simulation Variable Transformers in Laboratory Setup 


\subsection{Conclusion}

This chapter has presented an in depth discussion on the hardware and software used in the experimental setup. The thinking behind the design of the flexible test rig design was discussed. The back-to-back converter and its components were examined as well as the measurement and instrumentation hardware. An overview of the system protection was presented and the selection of the turbine emulator equipment was discussed. Finally the equipment that was used to implement supply voltage unbalance was explained. 


\section{CHAPTER 7}

\section{DISCUSSION OF EXPERIMENTAL RESULTS}

The experimental results are presented and discussed in this chapter. Reference is made to the simulation results when analysing the results. The experimental setup used to obtain these results was discussed in chapter 6. The generator and grid side converter step responses are investigated first followed by the operation of the grid side converter with an unbalanced supply. The effect of active damping on the grid phase currents is explored and finally the complete system operation under turbulent conditions is investigated.

\subsection{Generator Side Converter}

The generator side of the converter was tested by initially establishing a stable DC link voltage with the grid side converter. The operation of the two converters are independent of one another and therefore the grid side converter would have no effect on the results obtained in this section. A step response of the current and speed loops were performed in order to validate the correct operation of the system.

\subsubsection{Current Step Response}

The current step response was performed during a blocked rotor test for the same reasons discussed in section 5.2.1. The rotor was blocked using a clamp and the q-axis current $I_{q}$ was stepped from a value of $0 A$ to $-1 A$. Only a small step was implemented for safety purposes. The torque produced by the generator is proportional to $I_{q}$ and a smaller torque would prevent the clamp from coming loose. Figure 99 illustrates the q-axis current step response of the generator. 


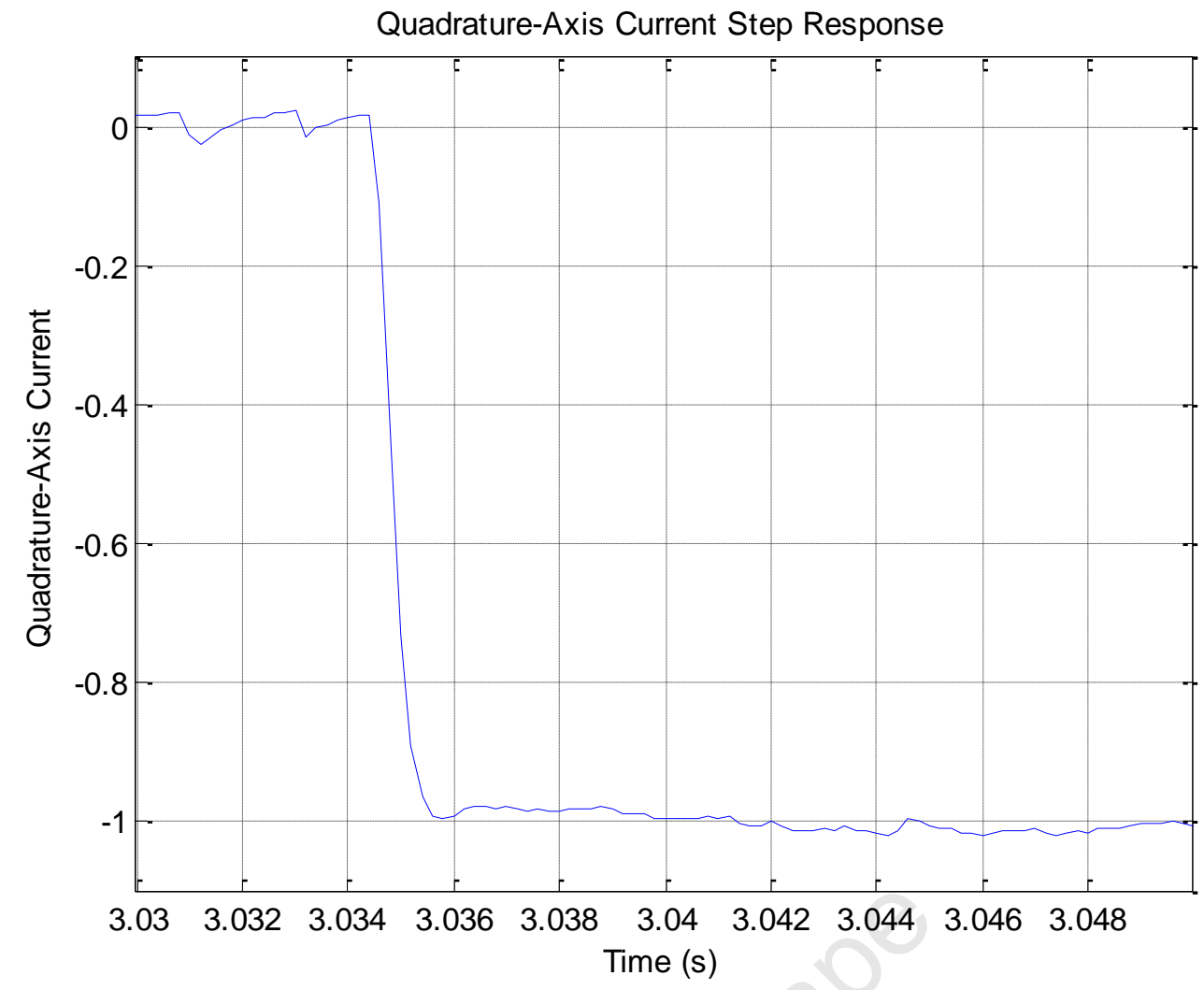

Figure 99: Experimental Generator Current Step Response

The settling time for a current step is $1 \mathrm{~ms}$ and no overshoot occurs. This is in the same time range as the simulated response time of $2 \mathrm{~ms}$, as shown in Figure 59 . The correlation of simulated and experimental results using the designed controller values indicates that the generator and control loop has been modelled correctly and that stable operation has been achieved.

\subsubsection{Speed Step Response}

The speed response test is performed by stepping the generator speed from $10.5 \mathrm{rad} / \mathrm{s}$ to $12.5 \mathrm{rad} / \mathrm{s}$ and is presented in Figure 100. 


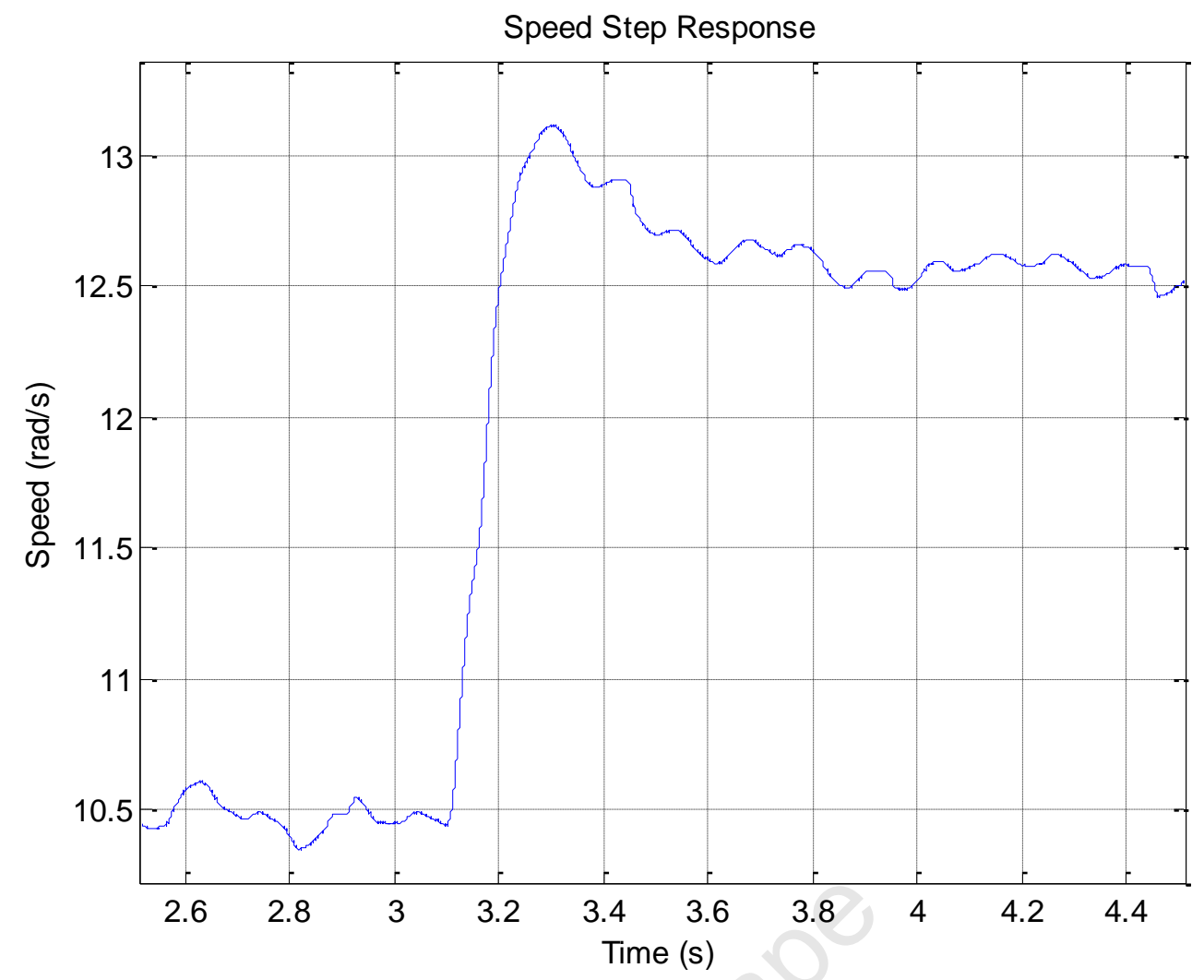

Figure 100: Experimental Speed Step Response

The speed step response has a settling time of 0.7 seconds and corresponds to the simulated settling time of the generator. An overshoot in the region of $25 \%$ is observed which differs from the simulated results of $15 \%$ in Figure 60 . This is a direct result of the delayed calculation of the generators speed which is not present in the simulations. The delay was discussed in section 6.3.2 and is also responsible for the steady-state speed having an oscillation in the region of $0.1 \mathrm{rad} / \mathrm{s}$. The response time of the generator speed is reasonable for wind turbine applications because of the relatively large timeframe over which turbulence occurs. This is confirmed in section 7.6 of this chapter.

\subsection{Grid Side Converter}

The experimental tests performed on the grid side converter were implemented by operation in rectifier mode. The step responses were tested to confirm the correct operation of the system. 


\subsubsection{Current Step Response}

Figure 101 below shows the step response of the current control loop which is shown in Figure 49. The negative current limit is reduced to $-2 A$ and the DC link voltage set point is stepped by a large amount so that the proportional gain will immediately cause the current set point to reach its limit.

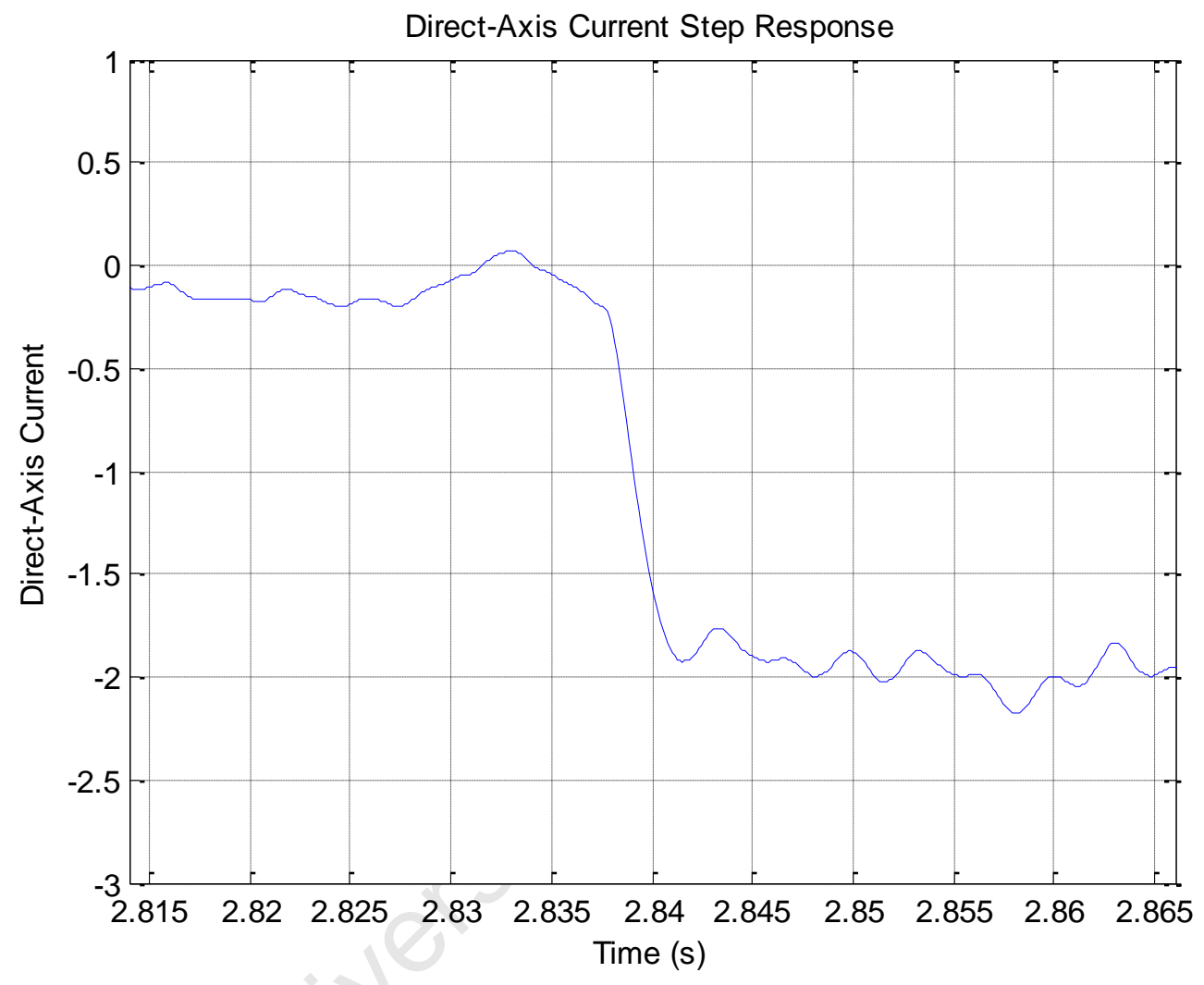

Figure 101: Experimental Grid Side Current Step Response

No overshoot occurs and the settling time is in the region of $5 \mathrm{~ms}$ which is the same as the simulation response time, as shown in Figure 61 . The correlation of simulated and experimental results using the designed controller values indicates that the grid filter and control loop has been modelled correctly and that stable operation has been achieved.

\subsubsection{Voltage Step Response}

The DC link voltage step response is presented in Figure 102. The voltage is stepped from $220 \mathrm{~V}$ to $260 \mathrm{~V}$. 


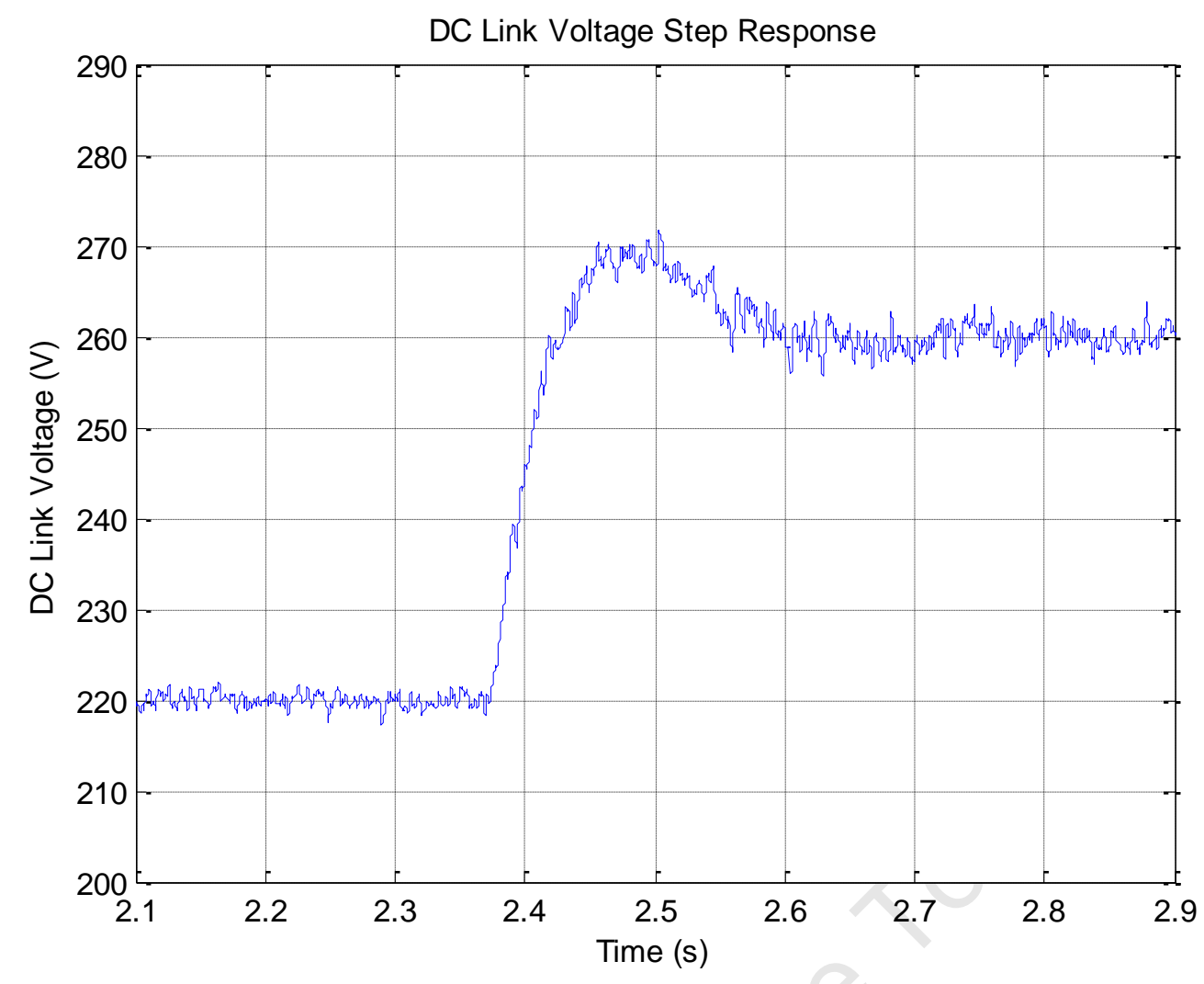

Figure 102: Experimental DC Link Voltage Step Response

A settling time $225 \mathrm{~ms}$ is observed with an overshoot of $25 \%$. The results are similar to the simulations in Figure 62 which had a settling time of $200 \mathrm{~ms}$ and an overshoot of $17 \%$. The difference in overshoot is due to the noise present in the experimental setup, that is not present in the simulations. The noise effects the DC link voltage control loop more than any of the other loops because it has a much larger scaling factor that it has to be multiplied by in the software in order to indicate the correct voltage, thereby amplifying any noise drastically.

\subsection{Turbine Effects}

The experimental results of the simulations conducted in section 5.2 are presented in this section. The full system was run in generation mode at a constant wind speed and connected to the grid. The experiments were conducted to show the effect that the turbine effects discussed in section 2.3 have on the generator parameters. 


\subsubsection{Experimental Effects of Wind Shear on the Generator}

The effects of wind shear on the generator parameters are illustrated in Figure 103. A slight sinusoidal shape is expected as discussed in section 5.2. Although the shaft power and torque have a sinusoidal shape, it is not at a frequency that could be caused only by wind shear. The sinusoidal variation is shape is probably due to the speed of the generator oscillating slightly about its reference value, causing the operating point to move away from the peak on the torque-speed curve which is shown in Figure 11. The wind shear effect is masked by this larger oscillation in torque.

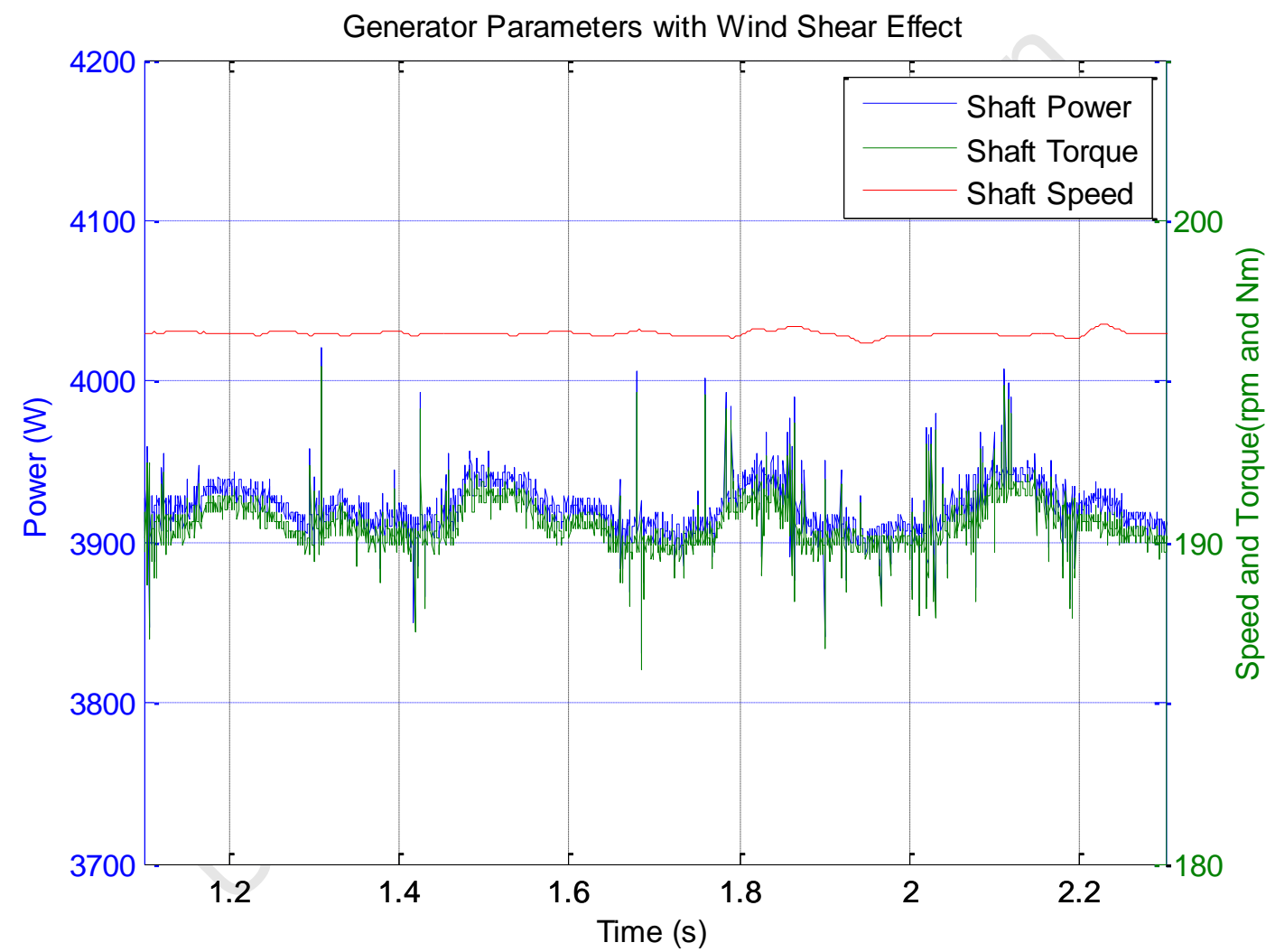

Figure 103: Experimental Effect of Wind Shear on Generator Parameters 


\subsubsection{Experimental Effects of Tower Shadow on the Generator}

Tower shadow is expected to have a more visible effect on the generator parameters compared to the wind shear effect as discussed in section 5.2. Figure 104 shows the generator parameters under the influence of the tower shadow effect. The dips are clear in the shaft torque and shaft power, occurring three times per revolution as expected. The dips are in the region of $6 \%$ which corresponds to the simulated value of $4 \%$ in section 5.2 . However, the shaft speed shows no signs of the dips. This is due to the calculation of the shaft speed which averages the speed over 10 calculations and is discussed further in section 6.3.2. The averaging has a smoothing effect when transients occur thereby masking the speed dips.

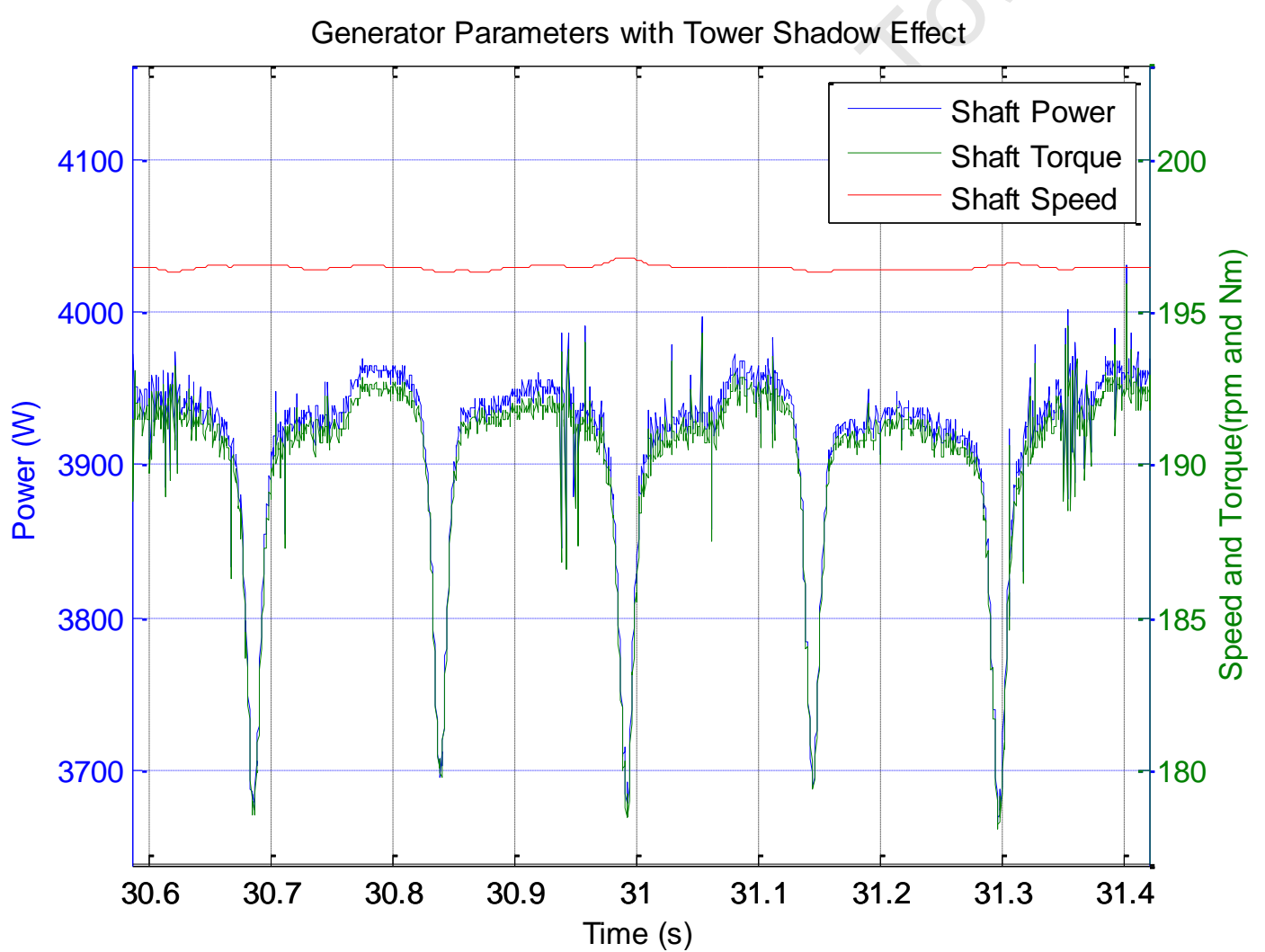

Figure 104: Experimental Effect of Tower Shadow on Generator Parameters 


\subsubsection{Experimental Effect on Generator with All Turbine Effects}

A combination of the two turbine effects discussed above is tested and the resulting effects on the generator parameters are shown in Figure 105 below. There is no visible difference between the results including all the turbine effects and the results of section 7.3.2. which only includes the tower shadow effect. This is due to the wind shear effect being masked by larger oscillations which are discussed in section 7.3.1.

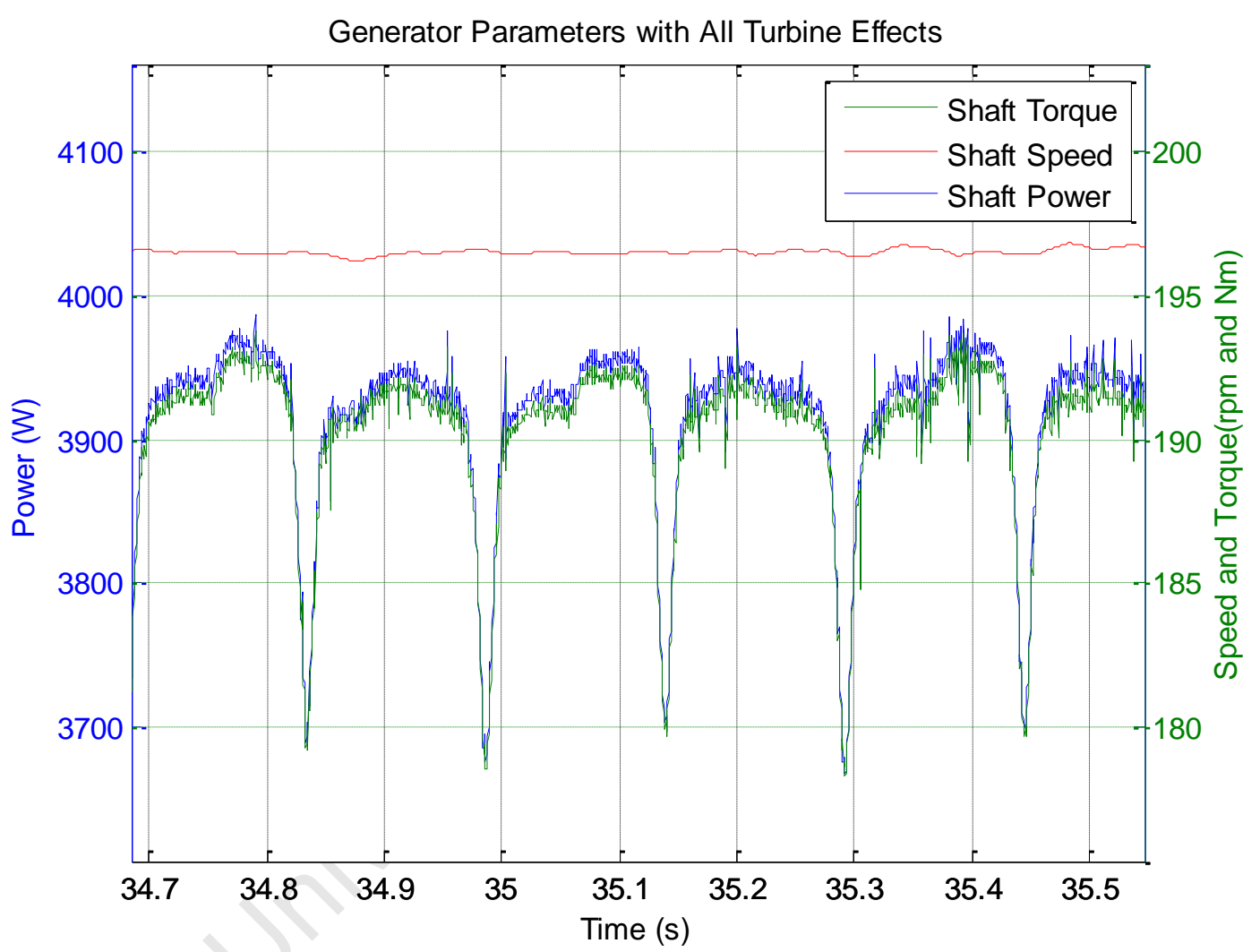

Figure 105: Experimental Effect on Generator of All Turbine Effects 


\subsection{Active Damping}

The effect of active damping on the grid phase currents was tested by running the experiment without active damping and then turning it on. Figure 106 shows the results that were obtained through this experiment. Active damping removes the resonant component that is introduced by the LCL filter when it is turned on at T $=1.855$ seconds and also stabilises the phase currents that were previously fluctuating. The fluctuations are caused by the resonant component which the $d q$ transformations are sensitive to. The effect of the resonance on the $d q$ currents is illustrated in Figure 107. The active damping is turned on and off periodically and it can be observed that the accuracy of the $d q$ currents suffers when no active damping is present.

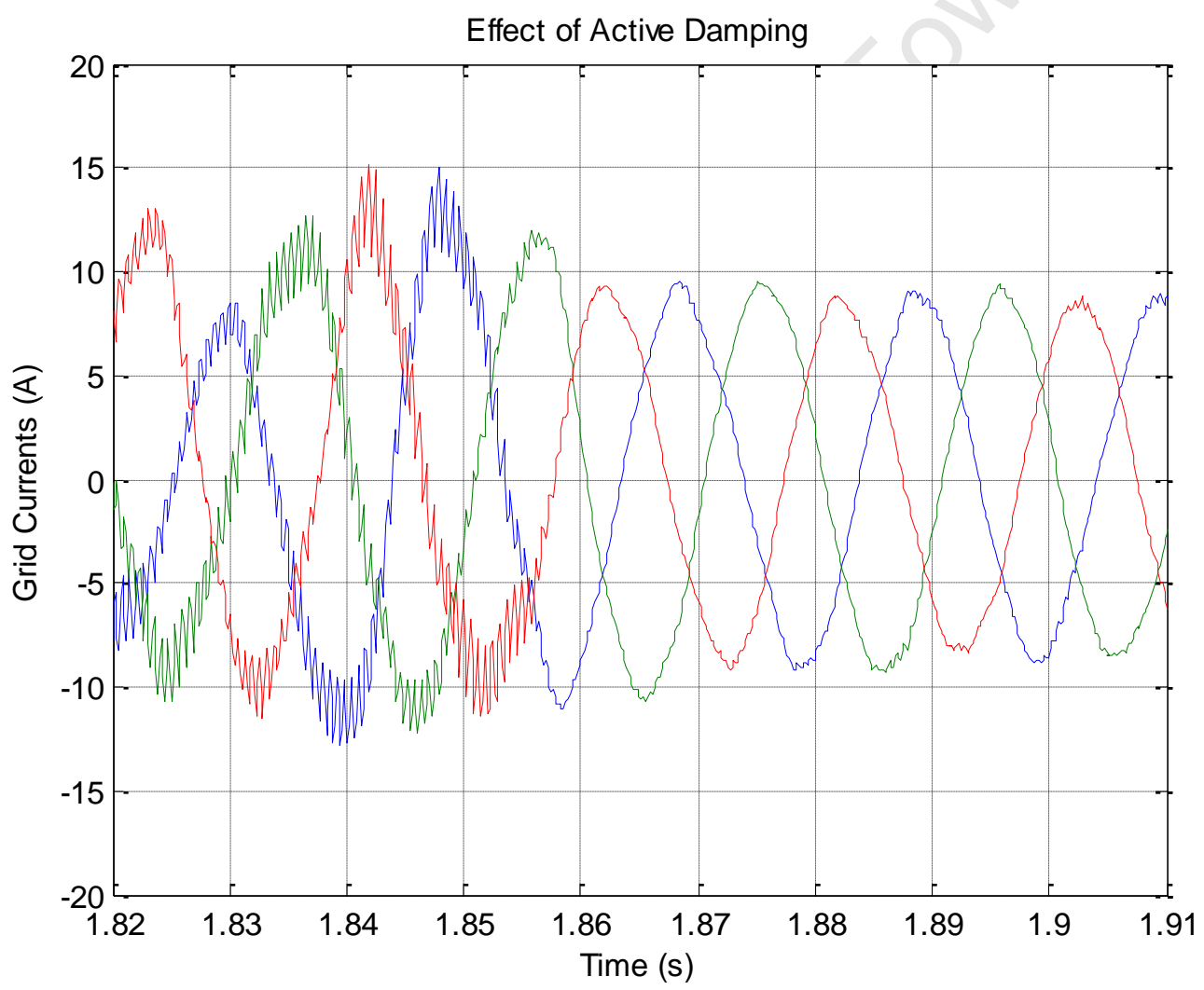

Figure 106: Effect of Active Damping on Grid Phase Currents 


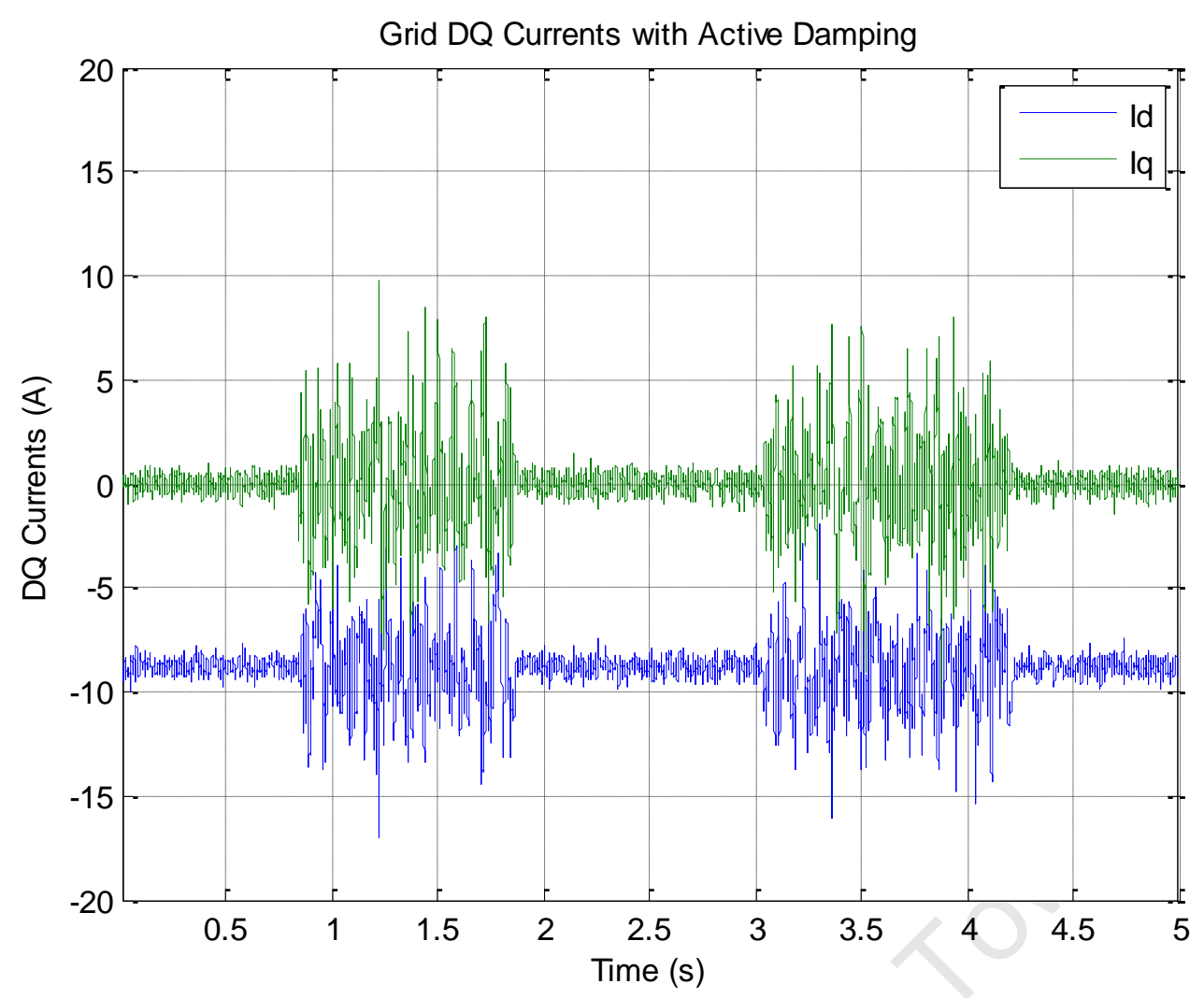

Figure 107: Grid DQ Currents with Active Damping Toggled On and Off

\subsection{Voltage Unbalance}

The standard and dual vector current control of the grid-side converter was implemented experimentally. Both techniques are tested under balanced grid conditions and a type B dip in order to assess their effectiveness. The dual vector current control is expected to produce balanced currents under all operating conditions. The two control techniques were tested under steady state conditions. This section presents the results obtained from testing the two control techniques. The experiments were conducted with the entire system running in generation mode at a constant wind speed with no turbine effects.

The grid phase voltages for balanced conditions and a type B dip are illustrated in Figure 108, Figure 109 below. The phase on which the dips occurs has a magnitude of $90 \%$ of its original value. The dip was implemented using the system in section 6.7 which is illustrated as a block diagram in Figure 97. The tests conducted under balanced grid voltages are used to check that the control is functioning correctly and to serve as a benchmark for ideal operating conditions. The UCT supply has always had 3rd and 5th harmonics present which tend to give the voltage waveforms a flatten peak characteristic as seen in the figures below. 


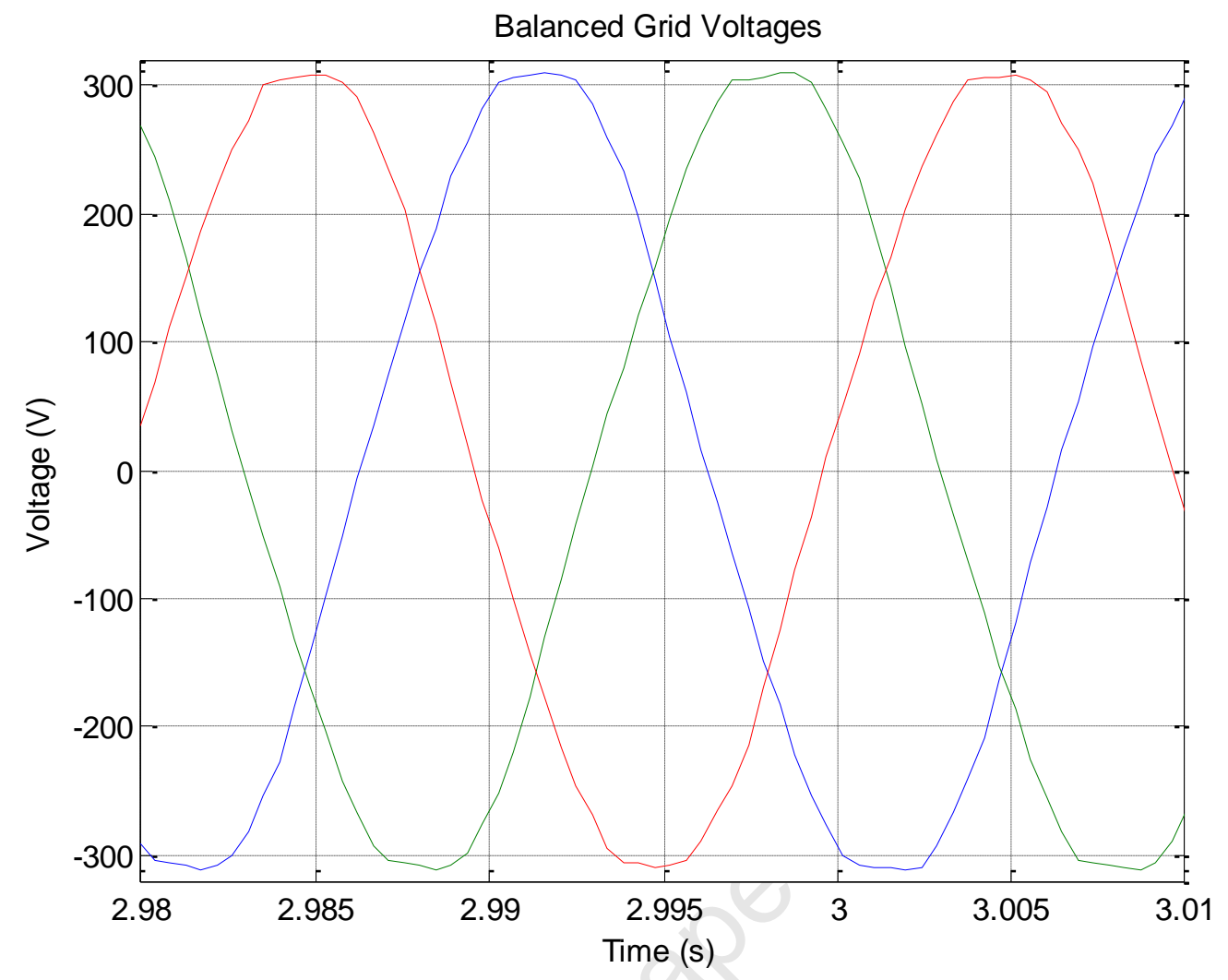

Figure 108: Balanced Grid Voltages

Grid Voltage with 10\% Type B Dip

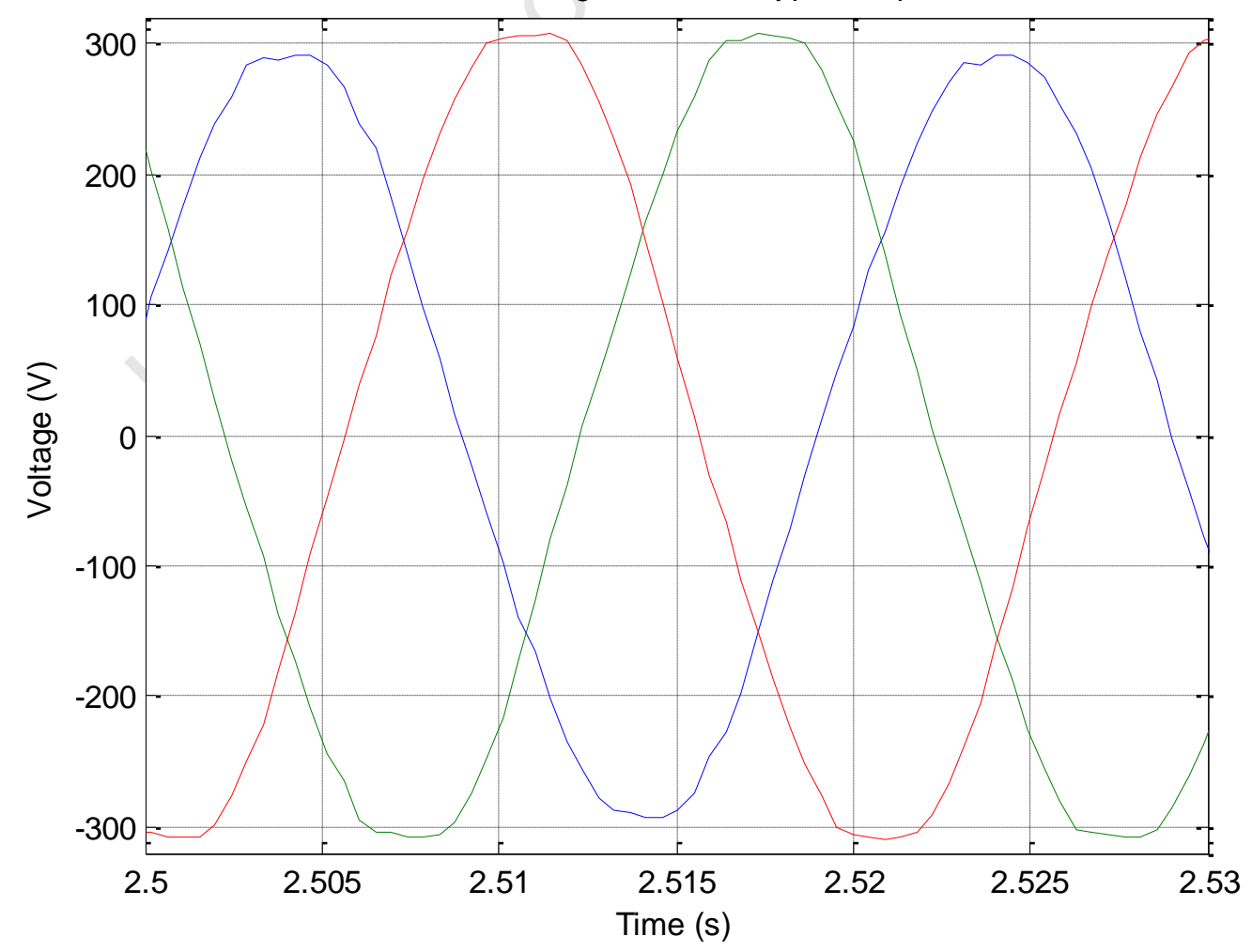

Figure 109: Grid Voltages With Type B Dip 


\subsubsection{Standard Vector Control}

The following set of results demonstrated the effects of voltage unbalance on standard vector control. This type of control uses the $d q$ currents to control the DC link voltage, transferring any excess power to the grid. UPF is ensured by forcing the q-axis current $I_{q}$ to zero and using the d-axis current $I_{d}$ to transfer the power. This control technique is discussed in section 3.3.

\subsubsection{Balanced Grid Voltages}

Figure 110 illustrates the grid phase currents that are achieved under balanced grid voltage conditions and Figure 111 shows the equivalent $d q$ currents. Unity power factor operation is achieved by keeping $I_{q}$ zero. The d-axis current $I_{d}$ remains fairly constant although there is a slight oscillation at a frequency of $100 \mathrm{~Hz}$, which indicates that there is a slight unbalance in the voltage or the impedance of the grid filter is not exactly the same in each phase, causing the grid currents to not be perfectly balanced. The simulated results in Figure 66 and Figure 67 exhibit phase currents that are better balanced because of the use of ideal components which do not exist in reality. As a result the simulated $d q$ currents have less variation.

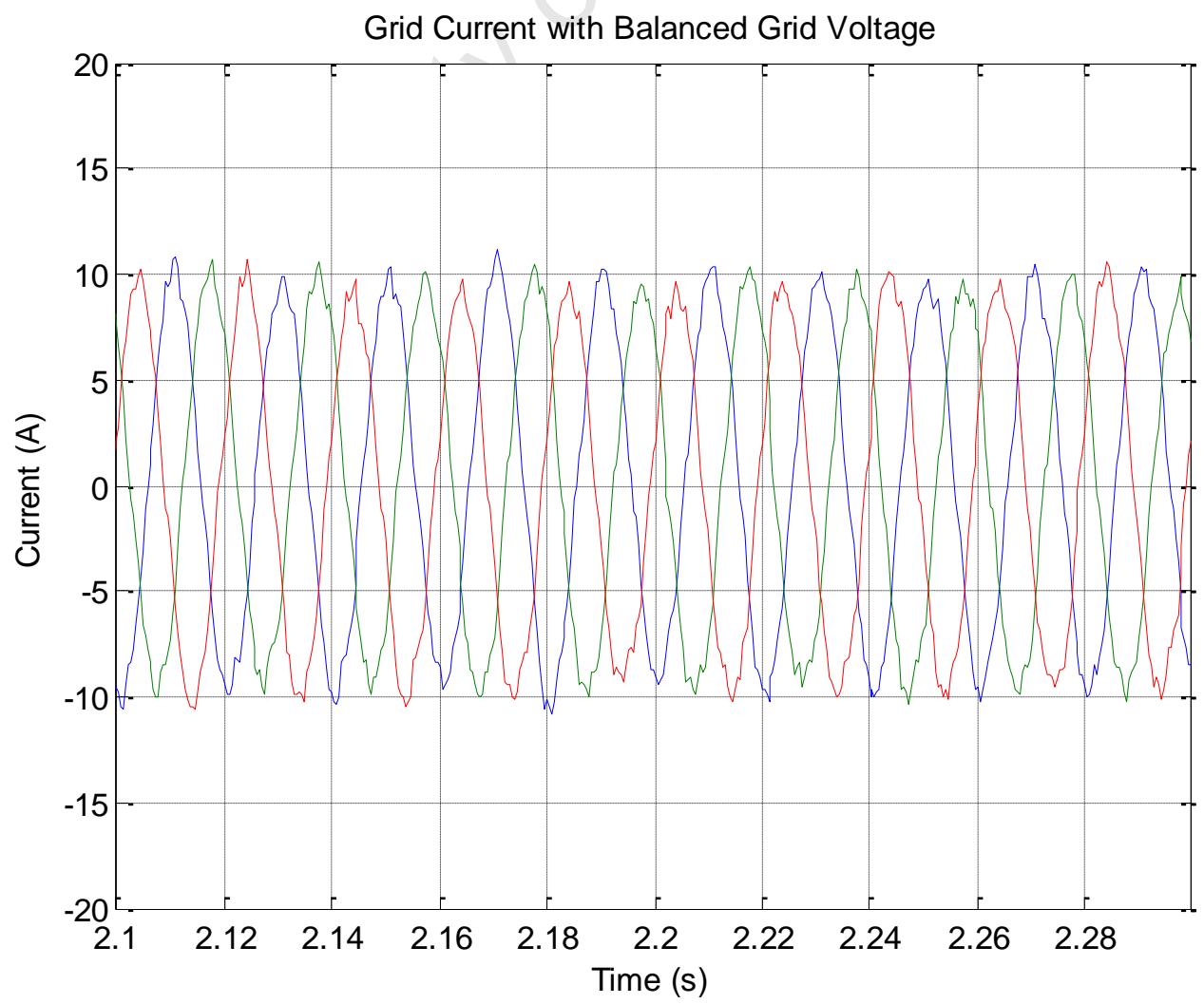

Figure 110: Experimental Grid Phase Currents Using Standard Vector Control with Balanced Grid Voltages 


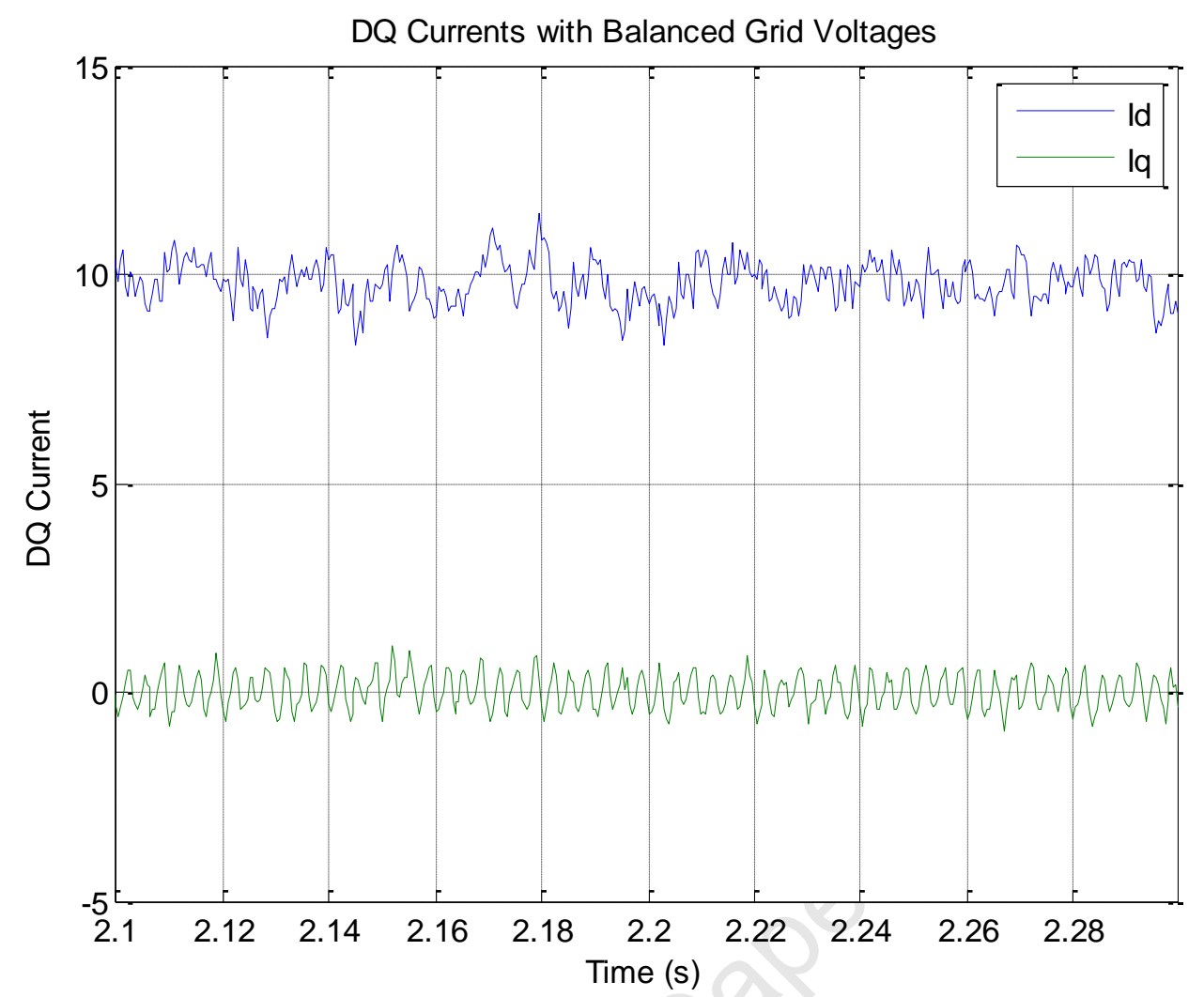

Figure 111: Experimental Grid DQ Currents Using Standard Vector Control with Balanced Grid Voltages

\subsubsection{Type B Dip}

The grid phase currents under a type B voltage dip as shown in Figure 109 are illustrated in Figure 112, whilst the corresponding $d q$ currents are illustrated in Figure 113. The grid currents are clearly unbalanced with one of the phases having a consistently higher magnitude than the other two phases. The q-axis current $I_{q}$ remains at zero but is expected to have a $100 \mathrm{~Hz}$ oscillation. $I_{d}$ has a $100 \mathrm{~Hz}$ component indicating that a negative sequence component is present. The presence of the $100 \mathrm{~Hz}$ component is expected and was discussed in section 3.5. These results correspond well with the simulated results in Figure 68 and Figure 69 although the same problems are present that were discussed in the previous section 7.6.1.1 leading to variation in the $d q$ currents. This causes the $100 \mathrm{~Hz}$ component to be masked slightly. 


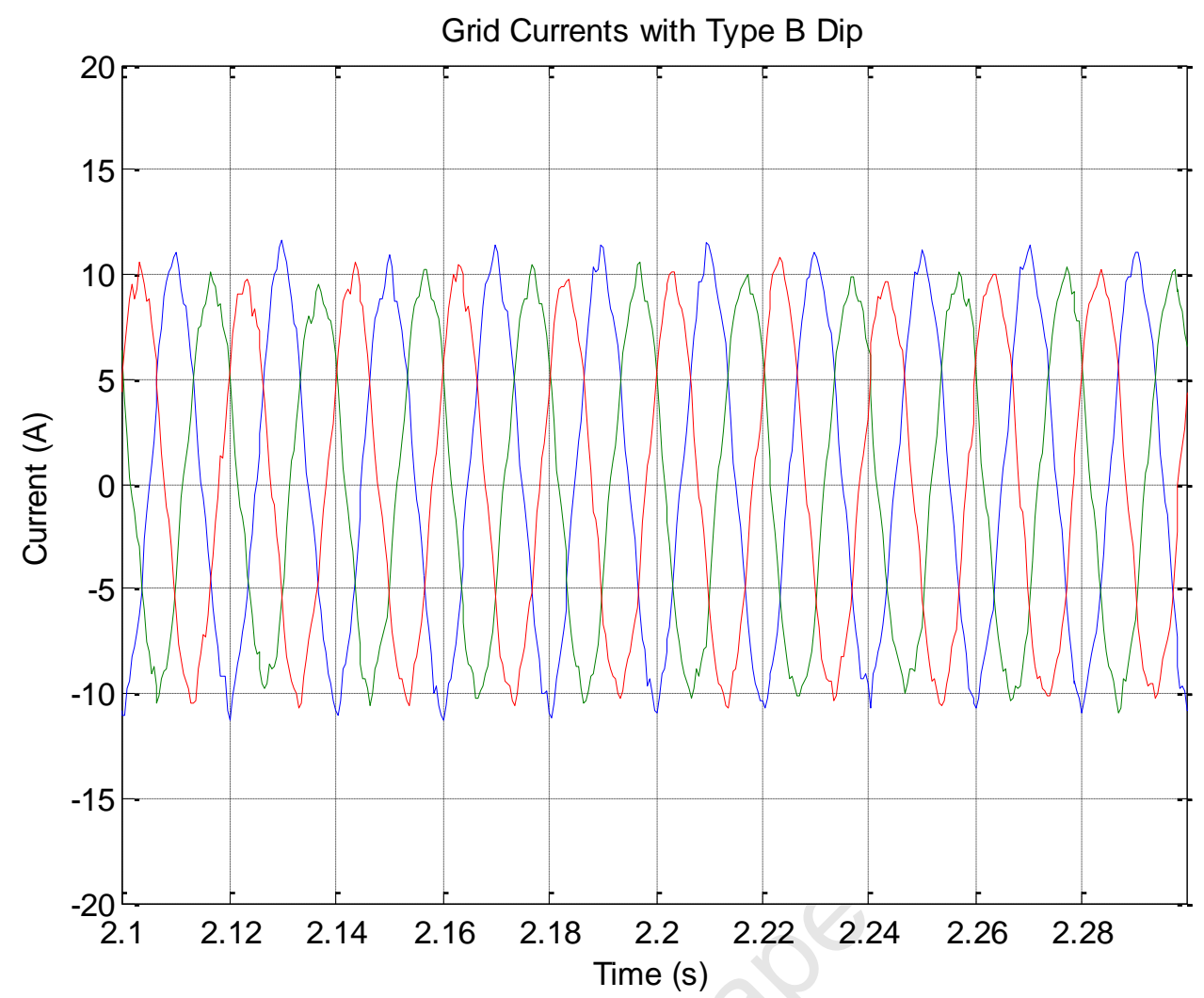

Figure 112: Experimental Grid Phase Currents Using Standard Vector Control with Type B Dip

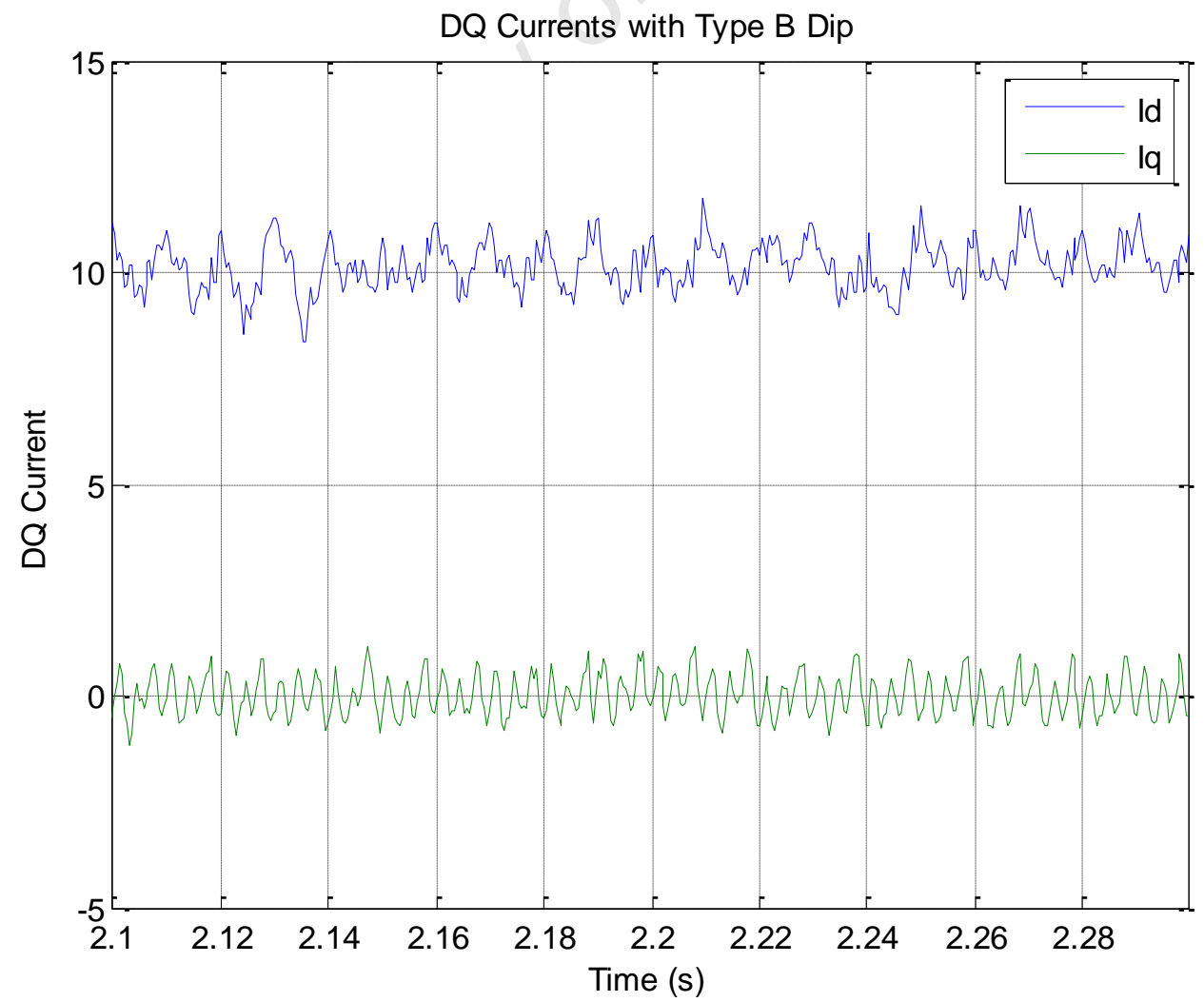

Figure 113: Experimental Grid DQ Currents Using Standard Vector Control with Type B Dip 


\subsubsection{Dual Vector Current Control}

The following set of results demonstrated the effects of voltage unbalance on dual vector current control. This type of control uses the positive and negative sequence $d q$ current components to control the DC link voltage, transferring any excess power to the grid. UPF is ensured by forcing the positive sequence q-axis current $I_{q+}$ to zero and using the positive sequence $\mathrm{d}$-axis current $I_{d+}$ to transfer the power. The negative sequence current components are forced to zero in to produce balanced grid phase currents under unbalanced supply voltage conditions. This control technique is discussed in section 3.5.

\subsubsection{Balanced Grid Voltages}

Figure 114 presents the grid phase currents under balanced grid voltages as shown in Figure 108. The currents remain fairly balanced compared to the standard vector control currents. A difference between the two control methods is noticed when inspecting the positive sequence direct-axis current $I_{d+}$ in Figure 115 which seems to have a more stable average value but has a fairly large, constant ripple. The ripple is due to the transformations from the grid currents to the positive and negative sequence components which involves differentiation, which amplifies any distortion in the current waveforms. Figure 116 shows the negative sequence components that are forced to zero by the control action in an attempt balance the currents. 


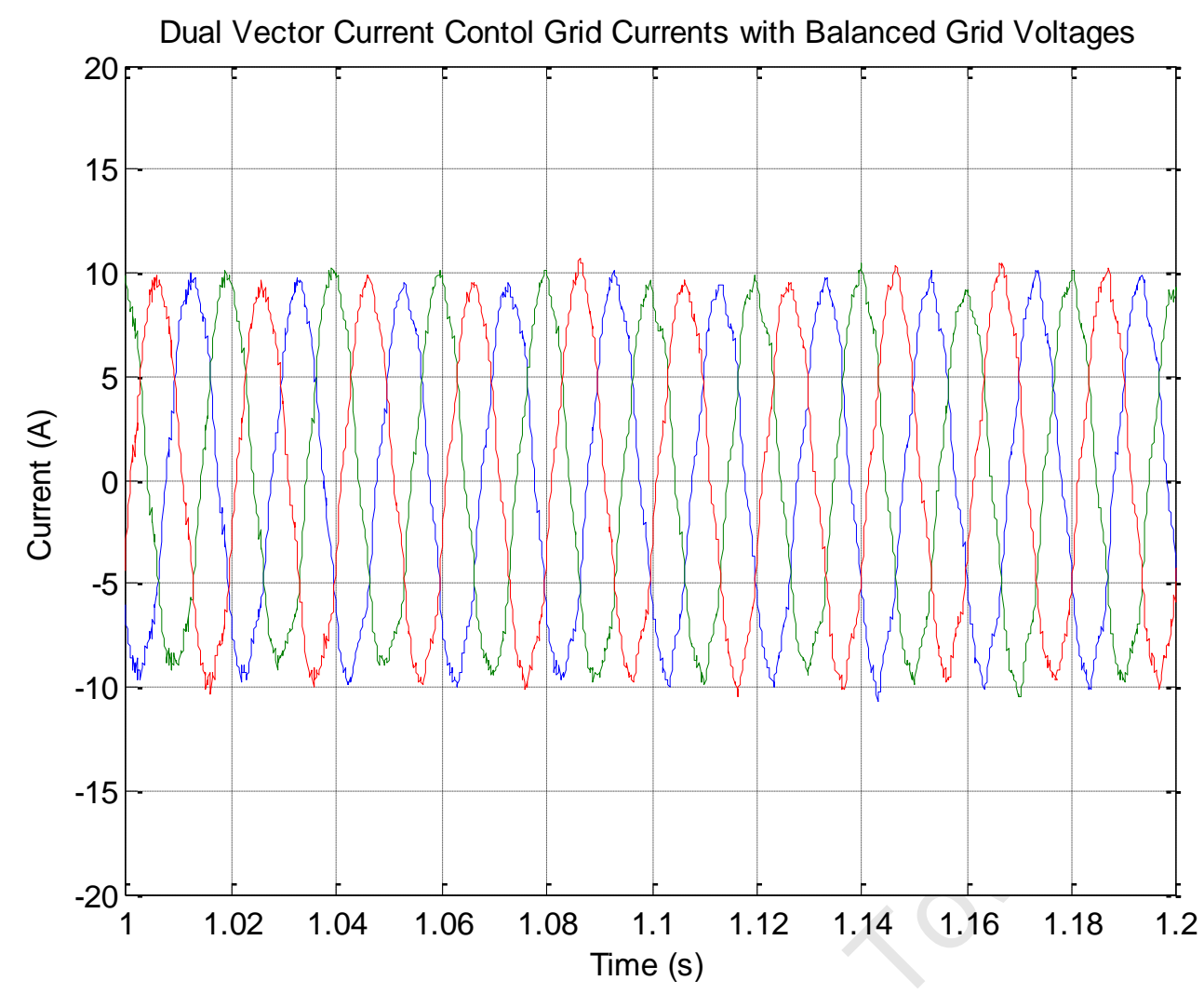

Figure 114: Dual Vector Current Control Grid Phase Currents with Balanced Grid Voltages

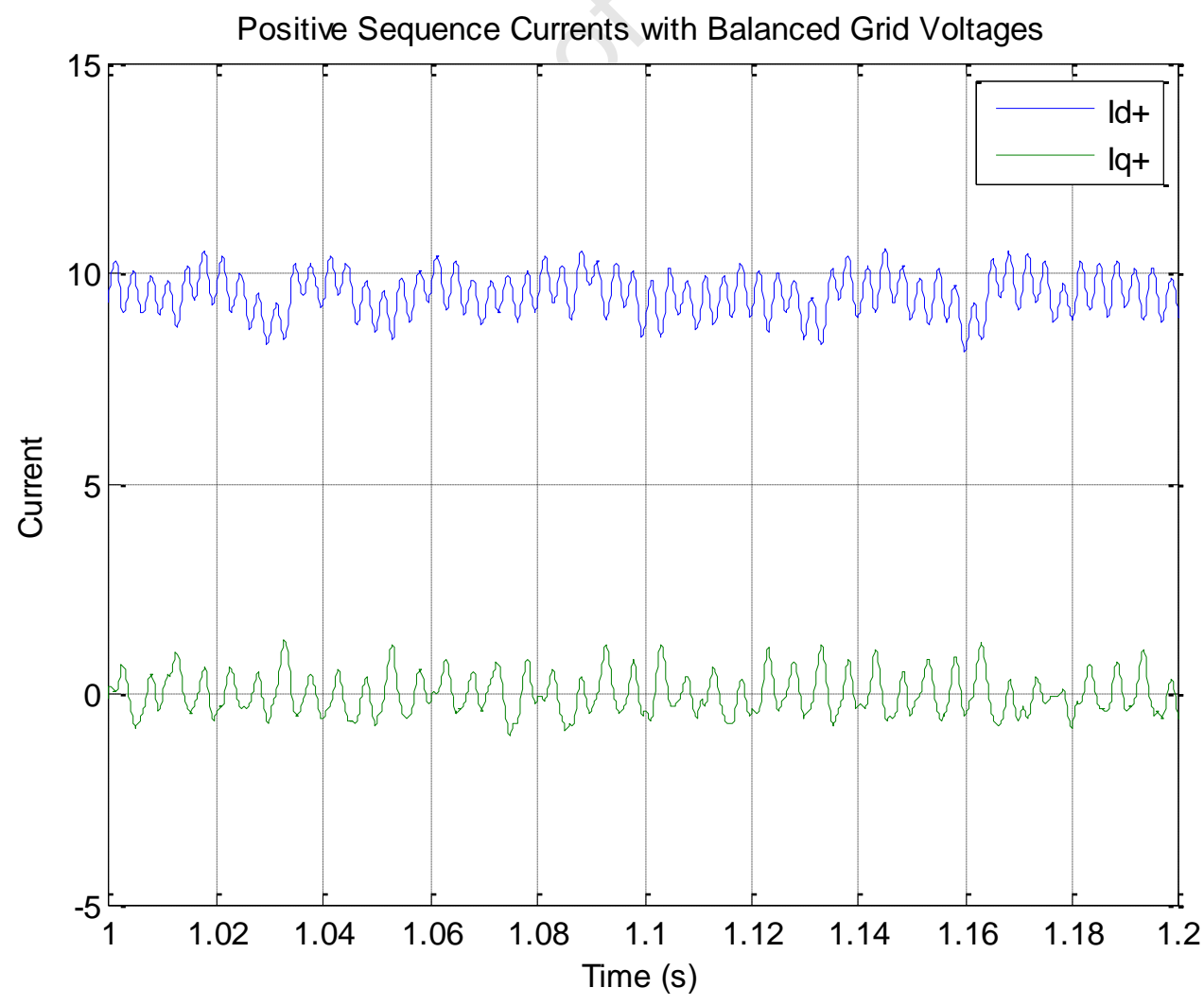

Figure 115: Dual Vector Current Control Positive Sequence DQ Currents with Balanced Grid Voltages 


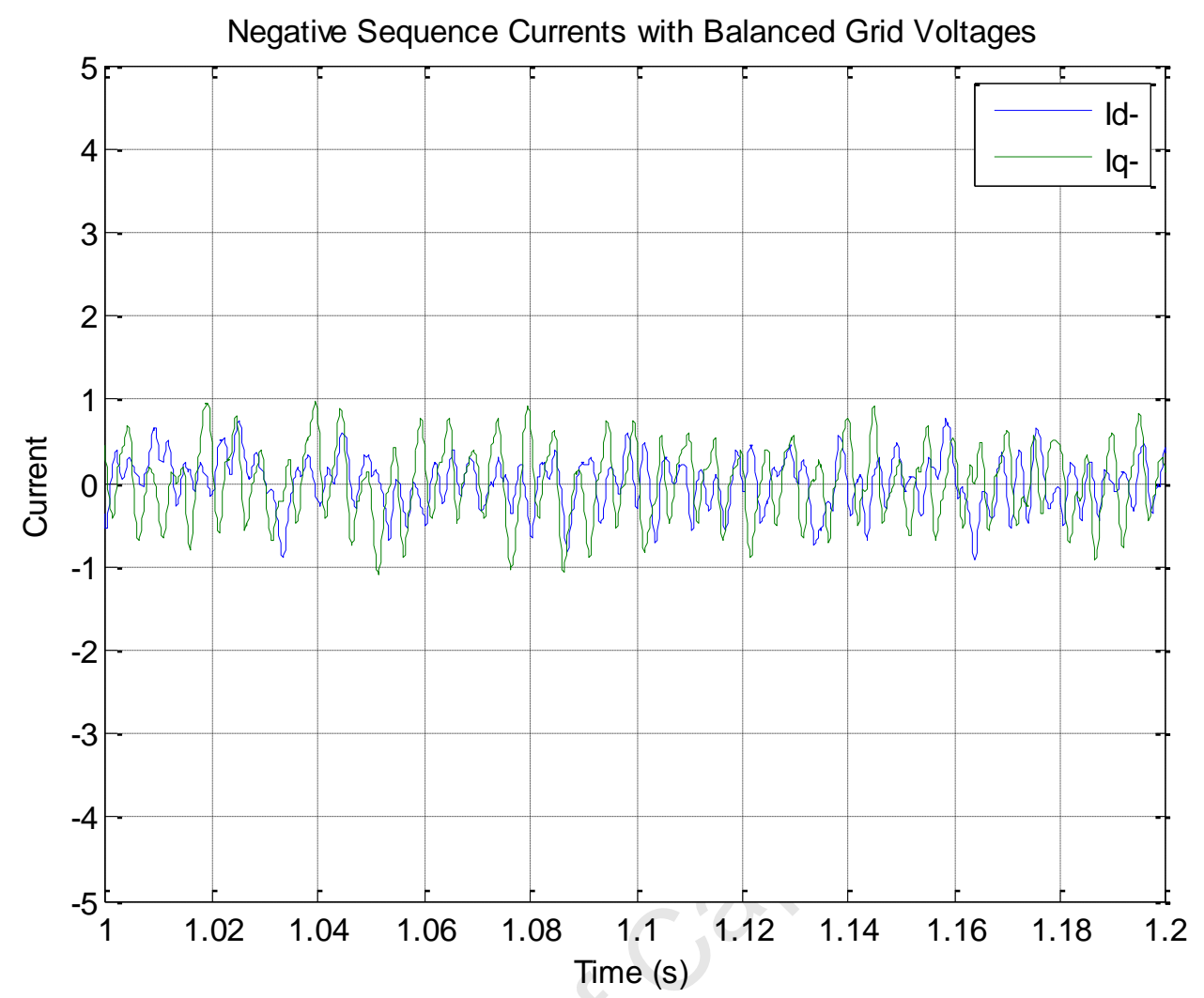

Figure 116: Dual Vector Current Control Negative Sequence DQ Currents with Balanced Grid Voltages

\subsubsection{Type B Dip}

Figure 117 shows the grid phase currents under a type B dip. The currents are balanced compared to the case where standard vector control was used. Figure 118 and Figure 119 present the positive and negative sequence components of the grid currents respectively. All of the sequence components are constant values with hardly any of the $100 \mathrm{~Hz}$ component present. These results are consistent with the simulations in section 5.5 where the dual vector current control was able to balance the phase currents under unbalanced supply voltage condition. 


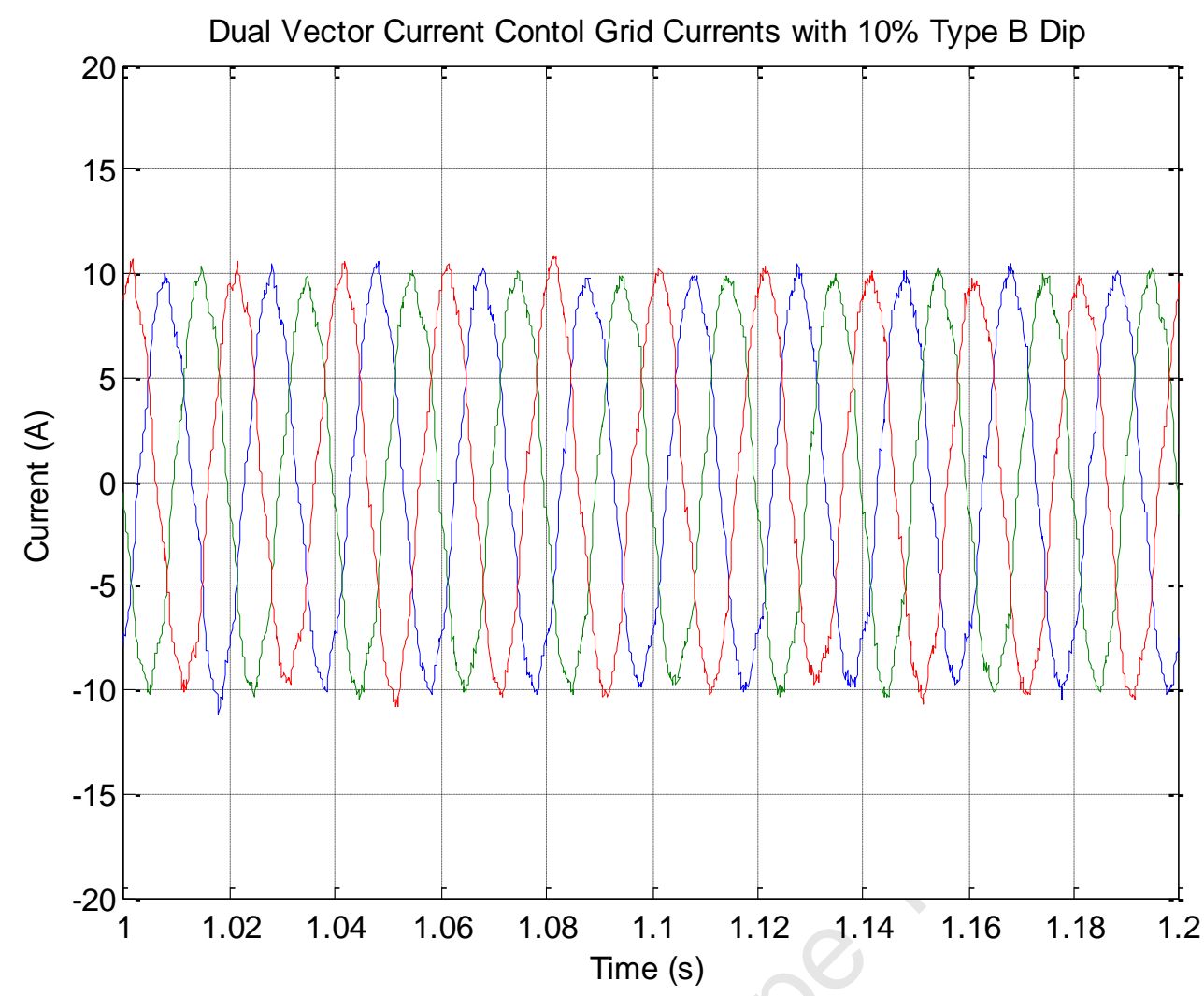

Figure 117: Dual Vector Current Control Grid Phase Currents with Type B Dip

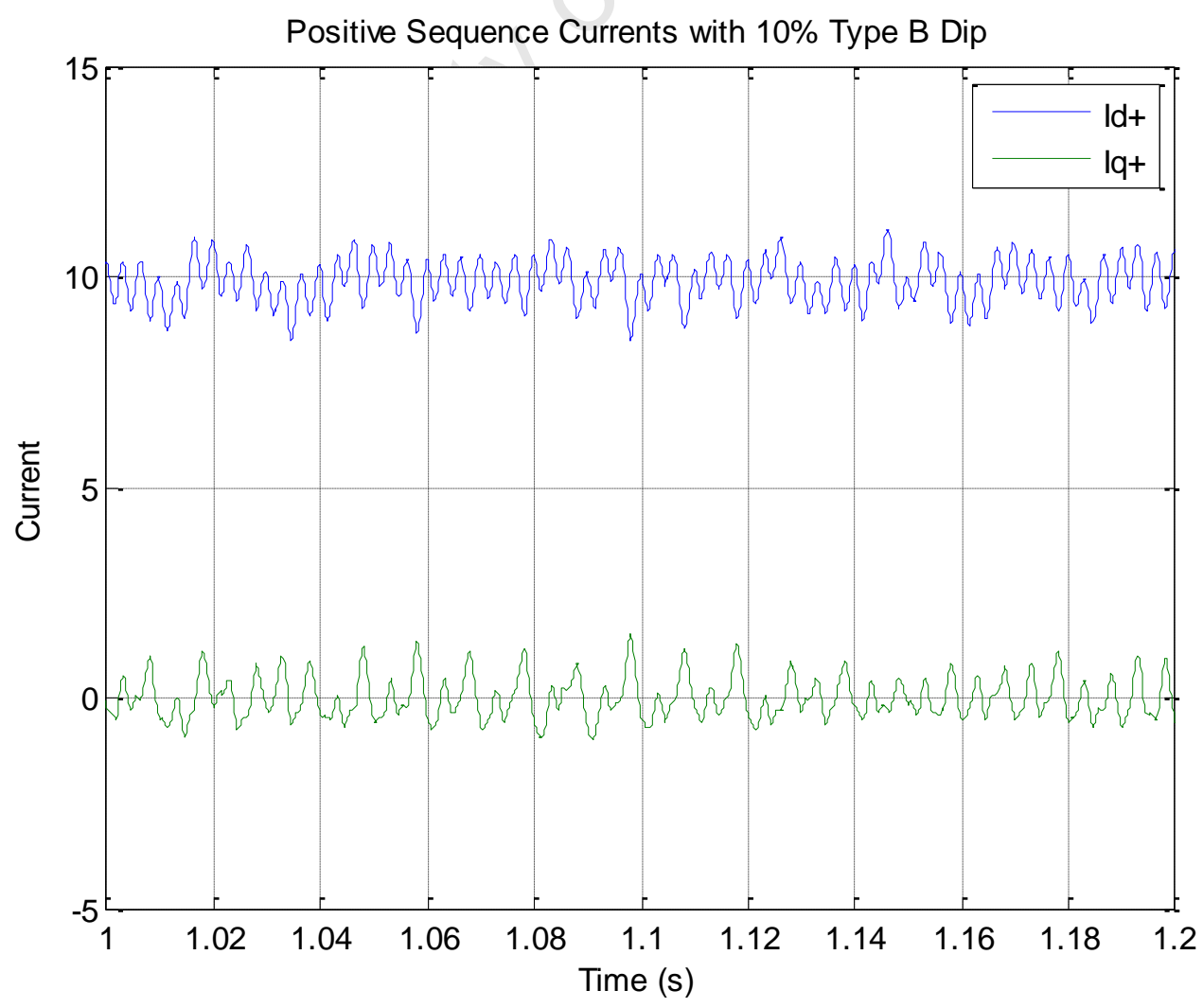

Figure 118: Dual Vector Current Control Positive Sequence DQ Currents with Type B Dip 


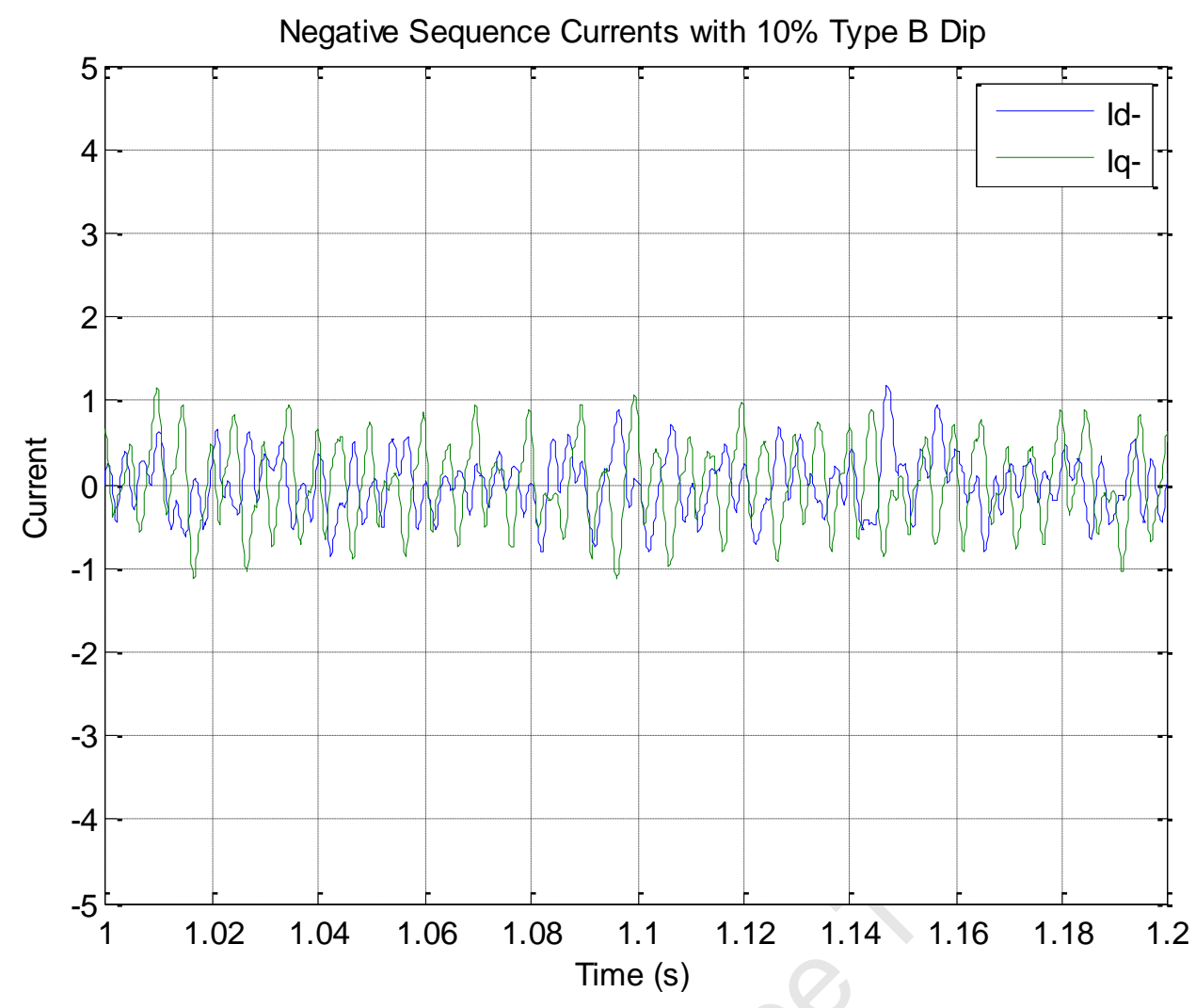

Figure 119: Dual Vector Current Control Negative Sequence DQ Currents with Type B Dip

\subsection{Full System Test}

A full system test was conducted using the turbulence model as well as all the turbine effects discussed in section 7.3. The goal of the experiment was to check that the system performed as expected under realistic conditions. Correct operation of the system using the two control strategies was proven in section 7.5 , therefore standard vector control was used with a balanced supply for simplicity.

Figure 120 presents the wind speed variations over a 120 second interval, with a nominal wind speed of $8 \mathrm{~m} / \mathrm{s}$. The turbulence characteristics modelled resemble that of a built up area and the wind speed varies rapidly between wind $5 \mathrm{~m} / \mathrm{s}$ to $10 \mathrm{~m} / \mathrm{s}$. 


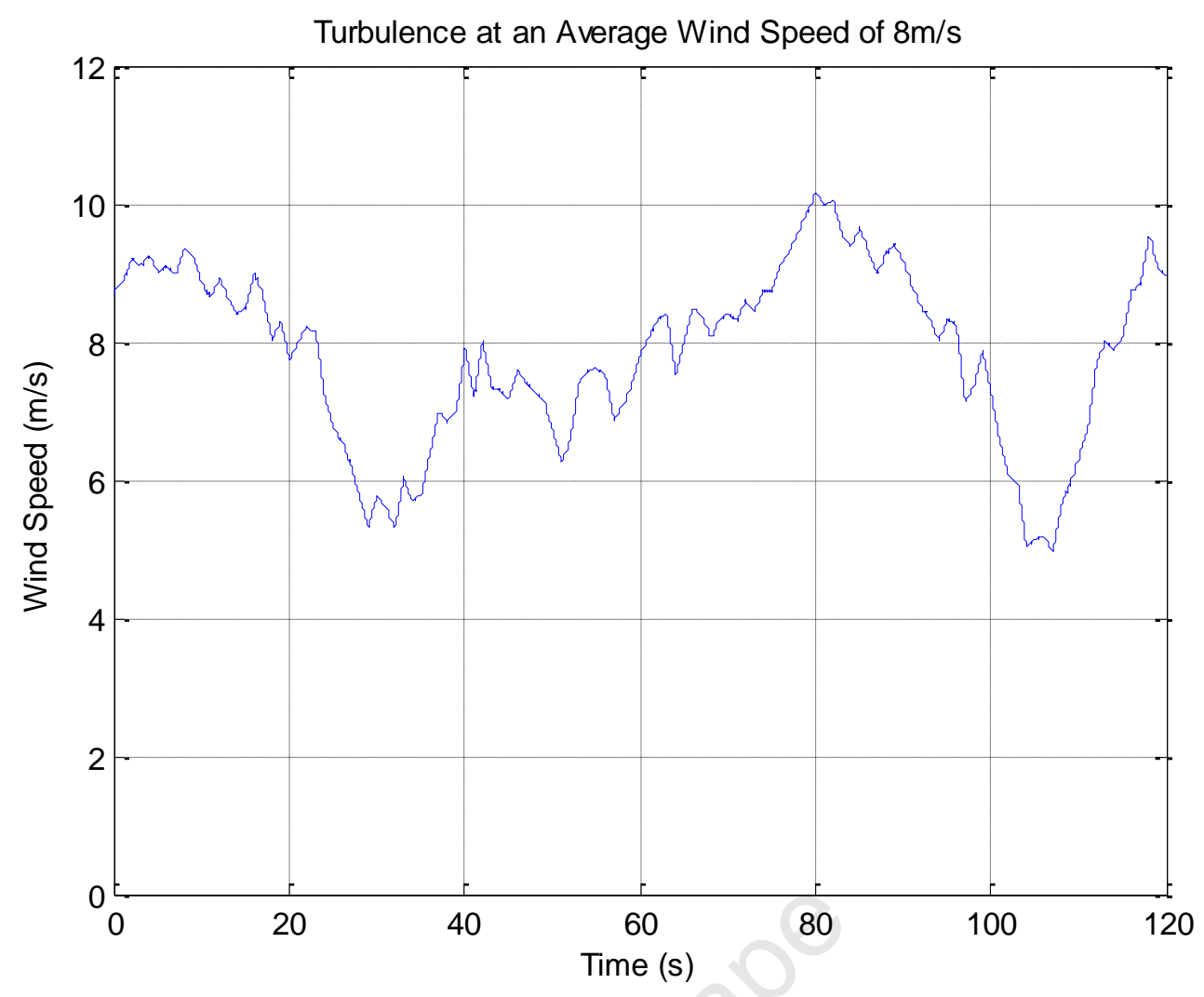

Figure 120: Experimental Wind Speed Under Turbulent Conditions

Figure 121 shows a comparison of the actual speed of the generator and its reference speed that is calculated by the MPPT algorithm. Figure 122 is zoomed in on a section of Figure 121. The results indicate that the generator's speed tracks the reference accurately under the turbulent wind conditions. 


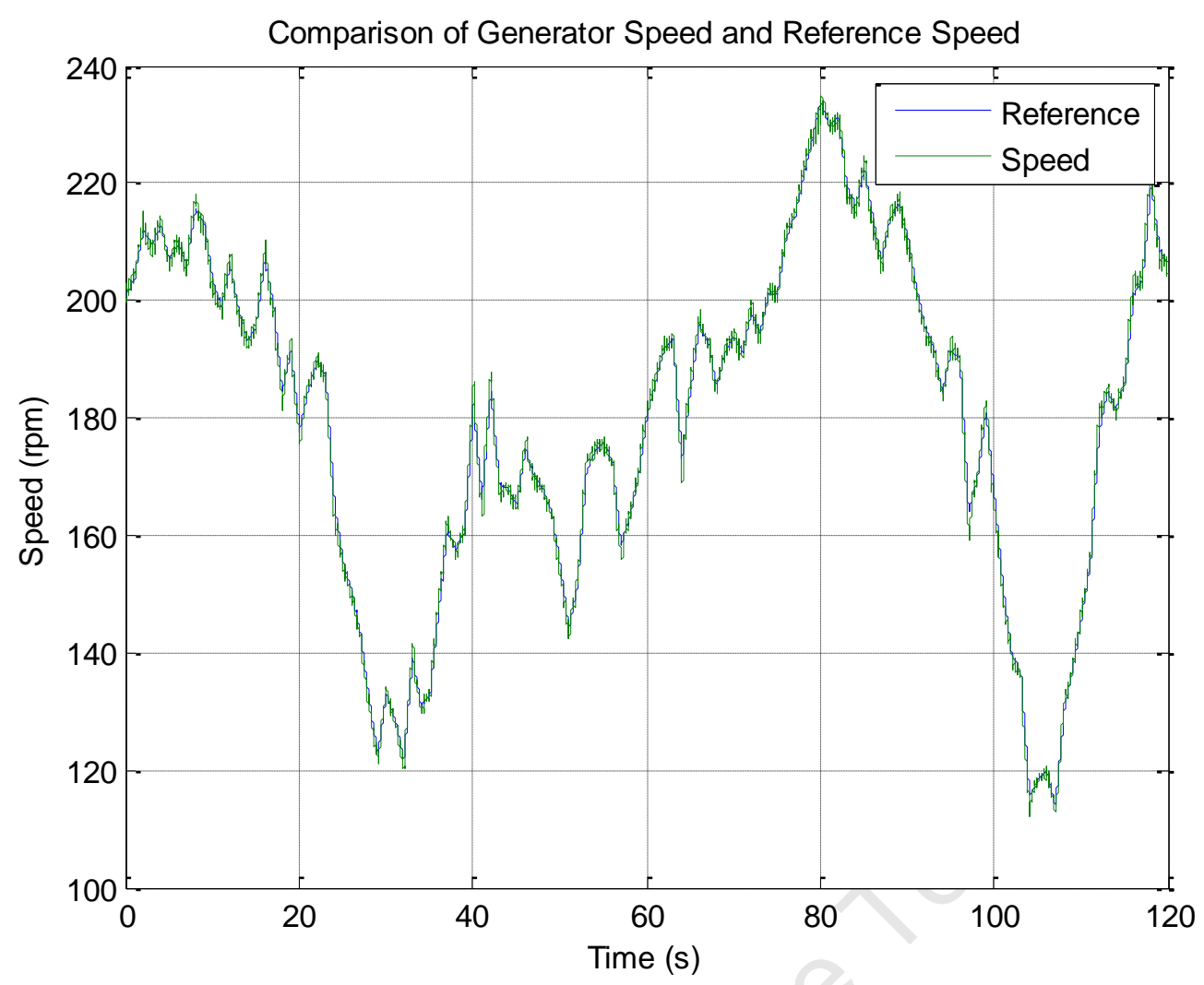

Figure 121: Comparison of Reference Speed with Actual Generator Speed

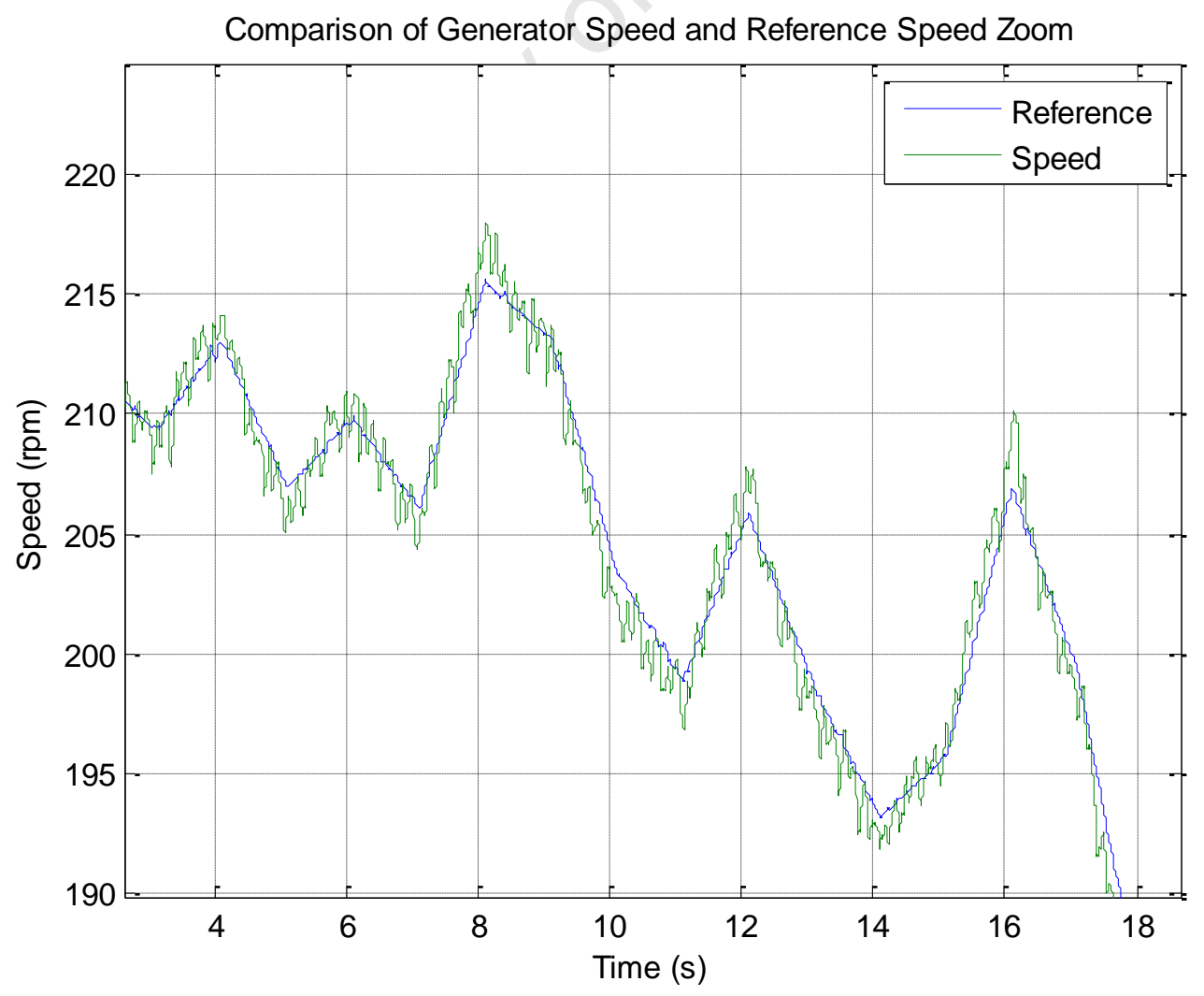

Figure 122: Close Up Section of Fig 116 
Further analysis of the effectiveness of the MPPT ability of the system can be conducted by inspecting the power coefficient results for the given time frame. Figure 123 indicates that $C_{p}$ has a maximum possible value of 0.4365 and the maximum deviation that occurs is 0.001 which means that $C_{p}$ is always within $0.23 \%$ of its maximum attainable value. The experimental result corresponds well to the simulated deviation of $0.17 \%$. Referring back to Figure 9 , a constant value of $C_{p}$ near its maximum value, indicates that the tip speed ratio is kept constant and at its optimum value.

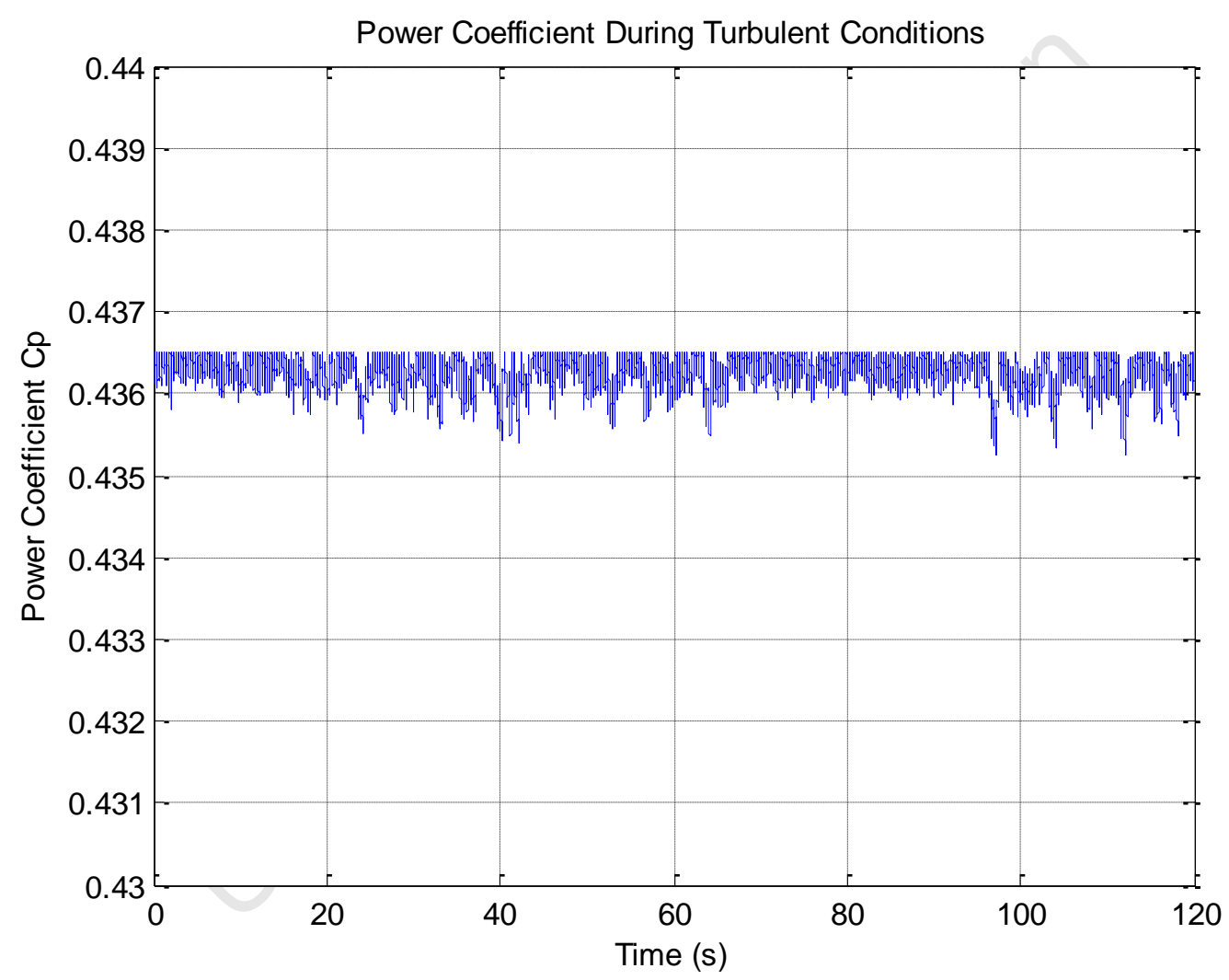

Figure 123: Experimental Power Coefficient Under Turbulent Conditions

Figure 124 shows the measured power at various stages of the system over the experiment time period. When the wind speed nears its rated value of $11 \mathrm{~m} / \mathrm{s}$ in Figure 120, the generator nears its rated speed of 240rpm in Figure 121, the shaft power approaches the rated value of $6 \mathrm{~kW}$. The generator power is always just below the shaft power because of the machine losses. The power transferred to the grid is in the region of $80 \%$ of the shaft 
power at all times. The rest of the losses are mainly incurred as follows: as switching losses in the back-to-back converter, across the discharging resistors on the DC Bus, and in the $\mathrm{LCL}$ filter components.

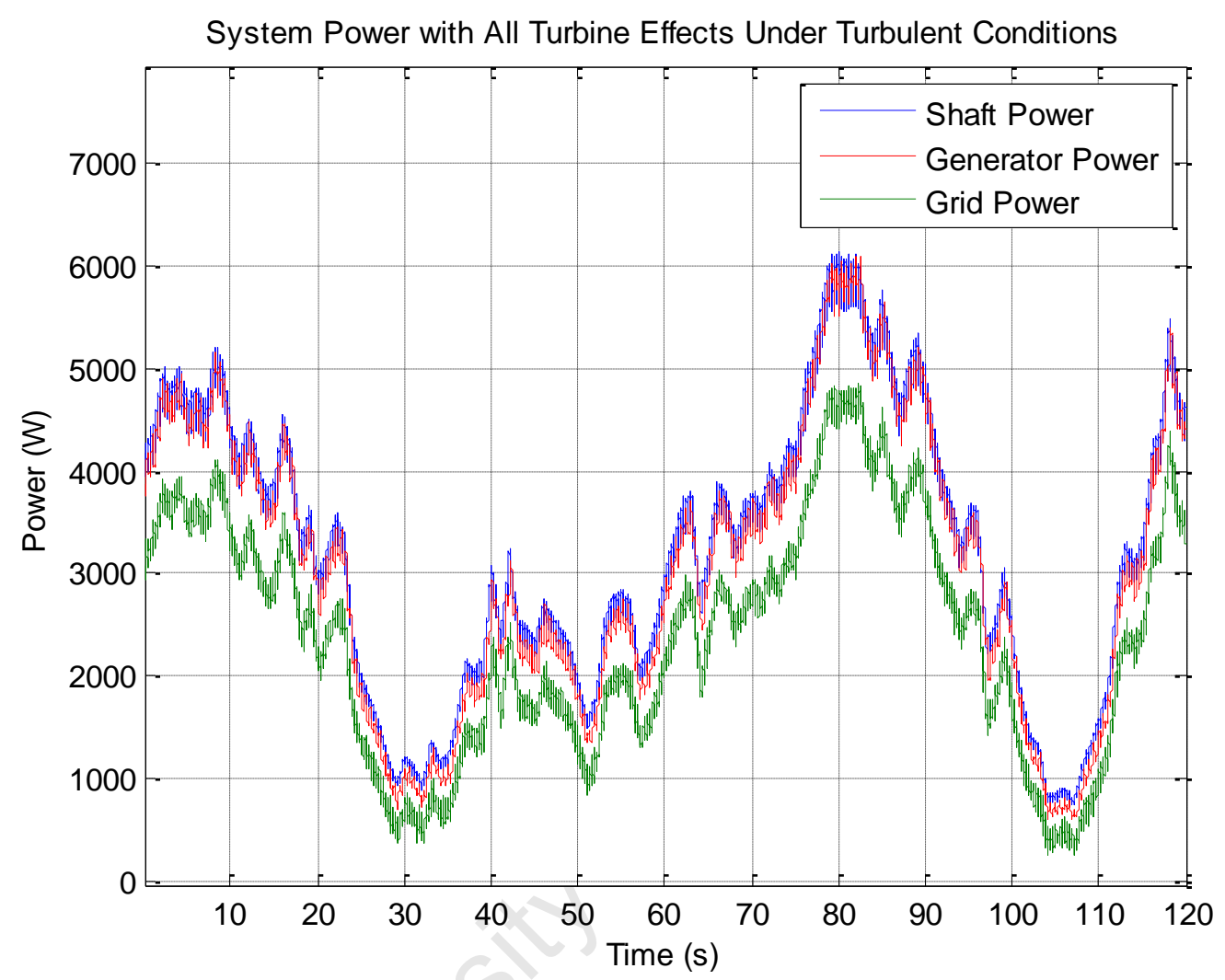

Figure 124: System Power at Various Stages During Turbulent Conditions

The DC link voltage is controlled by the grid side converter which is discussed in section 3.3. The time constant for the voltage step response in Figure 102 is less than that of the wind turbulence and the generator speed response. The control is therefore expected to keep the DC link voltage constant under all operating conditions. Figure 125 shows the DC link voltage under turbulent conditions with all the turbine effects included, and confirms that the control was able to keep the voltage at its reference value of $650 \mathrm{~V}$. 


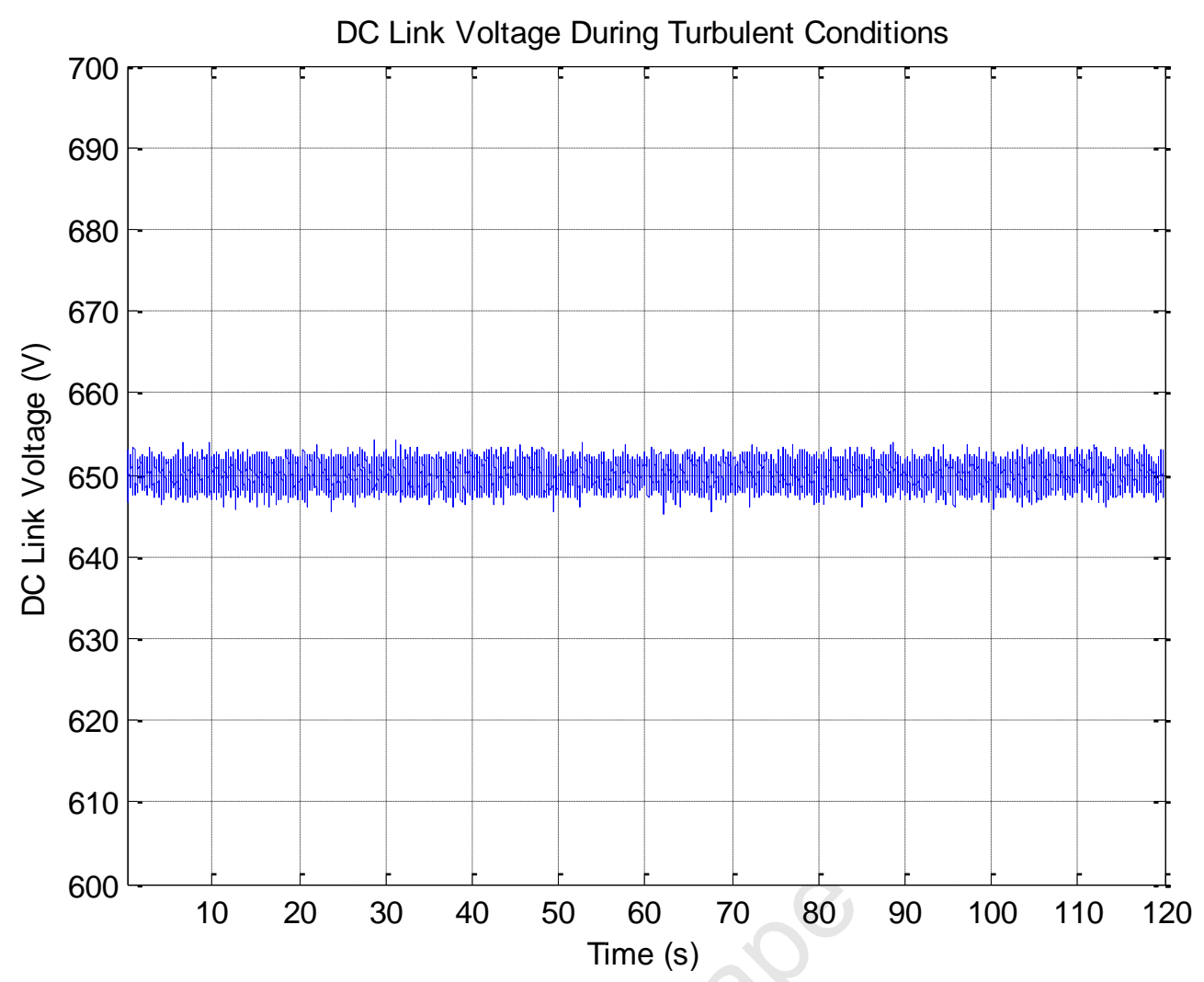

Figure 125: Experimental DC Link Voltage Under Turbulent Conditions

\subsection{Conclusion}

The different components of the system were tested experimentally in this chapter, in order to validate the systems performance. The experimental results validated the design and performance of the turbine emulator, generator and grid-side converters, as well as the MPPT algorithm. The grid-side converter was able to achieve balanced currents under unbalanced supply voltage conditions. It can be concluded that correct operation of the system was achieved with good correlation between experimental and simulated results. 


\section{CHAPTER 8}

\section{CONCLUSIONS AND RECOMMENDATIONS}

\subsection{Conclusions}

The design, analysis and testing of a grid-connected permanent magnet wind generation system was presented in this dissertation. The following conclusions can be drawn based on the findings presented in the dissertation:

- A model for emulating the turbine effects of wind shear and tower shadow was formulated. Additionally, a wind model of turbulence was also developed. The wind turbine emulator was simulated and implemented experimentally and the effects were validated through simulation and a set of experimental tests. The induction machine and VSD chosen for the wind turbine emulator were able to perform the required tasks without any problems. It can be concluded that the turbine emulator behaves as predicted in theory and has the expected effects on the operation of a WECS.

- A flexible test rig was designed and constructed in order to accommodate generators of varying sizes. The rig was able to hold the machine in place without any problems at the rated torque of $239 \mathrm{Nm}$.

- A model of the PMSG was developed in order to design the controllers for the generator control. The control of the machine has been discussed and investigated. The current and speed loop controllers were tested in simulation and experimentally. The results indicated that the model of the PMSG and control was accurate except for a varying delay that was produced by the encoder on the PMSG. It can be concluded that correct operation of the PMSG was achieved.

- A grid-connection filter was designed to eliminate the switching frequency current harmonics. The filter was included in the grid-side model used to design the controllers for the current and voltage control loops. The control loops were tested in simulation and experimentally. The results again indicate that the models are 
accurate but the noise that is introduced by the DSpace kits had to be taken into consideration when designing the voltage loop controller.

- The grid filter was found to introduce an oscillation at its resonant frequency in the grid currents. Various damping methods were investigated to eliminate the resonance and a sensorless band pass method was selected. The experimental results confirmed that the resonance was removed.

- The effect of grid unbalance on the system control was investigated. A different control strategy was adopted to deal with the current unbalance that was introduced into the system. It was tested in simulation as well as experimentally and was able to reduce the current unbalance.

- Turbulent wind conditions were tested in simulation and experimentally. It can be concluded from the results that the system control is able to operate effectively during such conditions.

\subsection{Recommendations}

- The system control should be implemented on an alternative DSP that has inputs and outputs that are isolated from earth to reduce the noise that is introduced into the system.

- A different method of determining the generator speed and position should be investigated that does not create a delay of a variable nature.

- The system should be connected to a grid emulator so that grid dips and swells can be introduced accurately on a shorter time scale.

- A control scheme that can operate under abnormal grid conditions should be implemented. 


\section{References}

[1] Yurika Ayukawa et al. Climate Solutions WWF's Vision for 2050. [Online]. assets.panda.org/downloads/climatesolutionweb.pdf

[2] (2010) Medium Term Risk Mitigation Plan (MTRM) for Electricity in South Africa - 2010 to 2016. [Online]. http://www.info.gov.za/view/DownloadFileAction?id=132959

[3] (2008) Electricity Pricing Policy (EPP) of the South African Electricty Supply Industry. [Online]. http://www.info.gov.za/view/DownloadFileAction?id=94201

[4] (2006) International Energy Agency Key World Energy Statistics. [Online]. http://www.iea.org/textbase/nppdf/free/2006/key2006.pdf

[5] (2010, October) Eskom Generations Plant Mix Revision. [Online]. http://www.eskom.co.za/content/GX 0001GenPlantMixRev11 1.pdf

[6] (2010) Executive Summary of the Draft Integrated Electricity Resource Plan for South Africa - 2010 to 2030 IRP 2010. [Online]. http://www.doeirp.co.za/content/Executive Summary Draft IRP2010 220ct2010.pdf

[7] K. Hagemann, "Mesoscale Wind Atlas of South Africa," University of Cape Town, Thesis presented for the degree of Doctor of Philosophy 2008.

[8] T.S. MOLINSKI D.L. HOFFMAN, "How New Technology Developments Will Lower Wind Energy Costs," in CIGRE/IEEE PES Joint Symposium Integration of Wide-Scale Renewable Resources Into the Power Delivery System, 2009.

[9] (2011) Special Report on renewable Energy Sources and Climate Change Mitigation. [Online]. http://srren.ipcc-wg3.de/

[10] Dr. Kurt Wiesegart et al. (2011) Options for the Establishment of a South African Wind Energy Centre (SAWEC) with Lessons Learnt from China and Germany. [Online]. 
http://green-cape.co.za/upload/SAWEC Report final draft June11.pdf

[11] Jay Rutovitz. (2010) South African Energy Sector Jobs to 2030. [Online]. http://www.greenpeace.org/africa/Global/africa/publications/climate/south-africanenergy-sector-jobs-to-2030.pdf

[12] Matthias Lange 1, KaiM“onnich, Hans-Peter Waldl, Hans Georg Beyer, Armin Luig Ulrich Focken. (2001) A statistical analysis of the reduction of the wind power prediction error by spatial smoothing effects. [Online]. http://www.mendeley.com/research/statisticalanalysis-reduction-wind-power-prediction-error-spatial-smoothing-effects/

[13] Syed Islam Kelvin Tan, "Optimum Control Strategies in Energy Conversion of PMSG Wind Turbine System without Mechanical Sensors," IEEE Transactions on Energy Conversion, vol. 19, no. 2, pp. 392 - $399,2004$.

[14] Shin-il Kang, Nyeon-Kun Hahm Seung-Ho Song, "Implementation and Control of Grid Connected AC-DC-AC Power Converter for Variable Speed Wind Energy Conversion System," in Eighteenth Annual IEEE Applied Power Electronics Conference and Exposition, 2003, pp. 154 - 158 vol.1.

[15] V. Dinavahi, A. M. Knight J. A. Baroudi, "A Review of Power Converter Topologies for Wind Generators," in IEEE International Conference on Electric Machines and Drives, 2005, pp. $458-465$.

[16] Gijs A.M. van Kuik. (2007) The Lanchester-Betz-Joukowsky Limit. [Online]. http://onlinelibrary.wiley.com/doi/10.1002/we.218/pdf

[17] Subbaraya Yuvarajan, Lingling Fan Xin Wang, "MPPT Control for a PMSG-Based GridTied Wind Generation System," in North American Power Symposium (NAPS), 2010, pp. $1-7$.

[18] S. Arnaltes, J.L. Rodriguez-Amenedo M. Chinchilla, "Laboratory set-up for Wind Turbine Emulation," in IEEE International Conference on Industrial Technology, 2004, pp. 553 557 Vol. 1. 
[19] Hiroki Goto, Hai-Jiao Guo, Osamu Ichinokura Kazmi Syed Muhammad Raza, "A Novel Algorithm for Fast and Efficient Maximum Power Point Tracking of Wind Energy Conversion Systems," in 18th International Conference on Electrical Machines, 2008, pp. $1-6$.

[20] Liuchen Chang Quincy Wang, "An Intelligent Maximum Power Extraction Algorithm for Inverter-Based Variable Speed Wind Turbine Systems," IEEE Transactions on Power Electronics, vol. 19, no. 5, pp. 1242 - $1249,2004$.

[21] Dragos Luca, Brayima Dakyo, Emil Ceanga Cristian Nichita, "Large Band Simulation of the Wind Speed for Real Time Wind Turbine Simulators," in IEEE Power Engineering Society General Meeting, 2002.

[22] P.W. Lehn Dale S.L. Dolan, "Simulation Model of Wind Turbine 3p Torque Oscillations due to Wind Shear and Tower Shadow," in IEEE PES Power Systems Conference and Exposition, 2006, pp. 2050 - 2057.

[23] Li Wang Tai-Her Yeh, "A Study on Generator Capacity for Wind Turbines Under Various Tower Heights and Rated Wind Speeds Using Weibull Distribution," IEEE Transactions on Energy Conversion, vol. 23, no. 2, pp. 592 - 602 , 2008.

[24] Grid Code Requirements For Wind Turbines Connected To Distribution Or Transmission $\begin{array}{llll}\text { Systems In South } & \text { Africa. }\end{array}$ http://www.nersa.org.za/Admin/Document/Editor/file/Electricity/TechnicalStandards/ RSA\%20Grid\%20Code\%20Connection\%20Requirements\%20for\%20Wind\%20Energy\%2 $\underline{\text { OFacilitie.pdf }}$

[25] Math H. Bollen, Understanding Power Quality Problems: Voltage Sags and Interruptions.: IEEE Press, 2000.

[26] P. Granjon, S. Bacha, F. Dumas V. Ignatova, "Classification and characterization of three phase voltage dips by space vector methodology," in International Conference on Future Power Systems, 2005. 
[27] Frede Blaabjerg, Steffan Hansen Marco Liserre, "Design and Control of an LCL-FilterBased Three-Phase Active Rectifier," IEEE Transactions On Industry Applications, vol. 41, no. 5, September/October 2005.

[28] Xiaoming Zha, Yan Zhou, Shanxu Duan, Fei Liu, "Design and Research on Parameter of LCL Filter in Three-Phase Grid-Connected Inverter," in Power Electronics and Motion Control Conference, 2009, pp. 2174 - 2177.

[29] Lan Xiao, Zhilei Yao, Chunying Gong, Xing Wei, "Design of LCL Filter for Wind Power Inverter," in World Non-Grid-Connected Wind Power and Energy Conference (WNWEC), 2010.

[30] Wu Xiaojie, Dai Peng, Zhou Juan, Sun Wei, "An Over View of Damping Methods for Three-phase PWM Rectifier," in IEEE International Conference on Industrial Technology, 2008.

[31] Pekik Argo Dahono, "A Control Method to Damp Oscillation in the Input LC Filter of ACDC PWM Converters," in IEEE 33rd Annual Power Electronics Specialists Conference, 2002.

[32] Anca Julean, "Active Damping Of LCL Filter Resonance In Grid Connected Applications," Aalborg University, MSc Thesis 2009.

[33] W. Szczygiel, M.P. Kazmierkowski, S. Bernet, M. Malinowski, "Sensorless Operation of Active Damping Methods for Three-Phase PWM Converters," in Proceedings of the IEEE International Symposium on Industrial Electronics, 2005, pp. 775 - 780.

[34] Antonio Dell'Aquila, Frede Blaabjerg, Marco Liserre, "Stability Improvements of an LCLfilter Based Three-phase Active Rectifier," in IEEE 33rd Annual Power Electronics Specialists Conference, 2002, pp. 1195 - 1201 vol.3.

[35] Chia-Tse Lee, Po-Tai Cheng, Che-Wei Hsu, "A Low Voltage Ride-Through Technique for Grid-Connected Converters of Distributed Energy Resources," in IEEE Energy Conversion Congress and Exposition (ECCE), 2010, pp. 3388 - 3395. 
[36] Jan Svensson, Giuseppe Saccomando, "Transient Operation of Grid-connected Voltage Source Converter Under Unbalanced Voltage Conditions," in IEEE Industry Applications Conference, 2001, pp. 2419 - 2424 vol.4.

[37] S. Busquets, J. Bordonau, J. Pontt, C. Silva, J. Rodríguez, S. Alepuz, "Fast On-Line Symmetrical Components Separation Method for Synchronization and Control Purposes in Three Phase Distributed Power Generation Systems," in European Conference on Power Electronics and Applications, 2007, pp. 1 - 10.

[38] K. Rafal, G. Abad, M. Jasinski, M. Bobrowska-Rafal, "Control of PWM rectifier under grid voltage dips," Bulletin Of the Polish Academy Of Sciences, vol. 57, no. 4, 2009.

[39] Araki M. CONTROL SYSTEMS, ROBOTICS, AND AUTOMATION - Vol. II - PID Control. [Online]. http://pdfebooks.studentbeatz.com/CONTROL-SYSTEMS,-ROBOTICS,-ANDAUTOMATION

[40] Marco Liserre, Pedro Rodríguez, Remus Teodorescu, Grid Converters for Photovoltaic and Wind Power Systems., 2011.

[41] Radu-Emil Precup, Stefan Preitl, "An extension of tuning relations after symmetrical optimum method for PI and PID controllers," Automatica 35, pp. 1731-1736, 1999.

[42] Martin Braae, Martin Machaba. Explicit Damping Factor Specification in Symmetrical $\begin{array}{lllll}\text { Optimum } & \text { Tuning } & \text { PI Online]. }\end{array}$ http://www.nt.ntnu.no/users/skoge/prost/proceedings/afcon03/Papers/050.pdf

[43] Ramu Krishnan, Electric motor drives: modeling, analysis, and control.: Prentice Hall, 2001.

[44] G.F Franklin, Feedback Control of Dynamic Systems, 5th ed.: Prentice Hall, 2009.

[45] A. helal, Y. Gabe,r H. A. Youssef, M. F. Moussa, "Unity Power Factor Control of Permanent Magnet Motor Drive System," in 12th International Middle-East Power System Conference, 2008, pp. 360 - 367. 
[46] Yi Tong, Yoji Takeda, Taka Hirasa, Shigeo Morimoto, "Loss Minimization Control of Permanent Magnet Synchronous Motor Drives," IEEE Transactions on Industrail Electronics, vol. 41, no. 5, October 1994.

[47] Stefan Sager, "Design and Implementation od a grid-connected variable-speed PM WECS," University of Cape Town, MSc Dissertation 2010. 


\section{APPENDIX A}

\section{Procedure 1:}

This procedure is used to align the index pulse of the encoder with the $d$ or $q$ axis of the rotor. Before the procedure is started, the encoder needs to be mounted on the shaft of the generator but the coupling grub screw must not be tight so that when the shaft is stationary, the encoder can still be rotated. When aligning the encoder, the Simulink program Encoder Align is used.

\section{Step 1:}

Turn the encoder so that the index is passed at least once. This orientates the encoder so that the index pulse is set as the zero count value.

Step 2:

Apply a DC voltage as indicated in figure () to align the rotor with either the $\mathrm{d}$ or $\mathrm{q}$ axis.

Step 3:

Position the encoder so that the count value is zero at this position and fasten the grub screw of the coupling to lock it in place. The encoder is now aligned to the chosen axis.

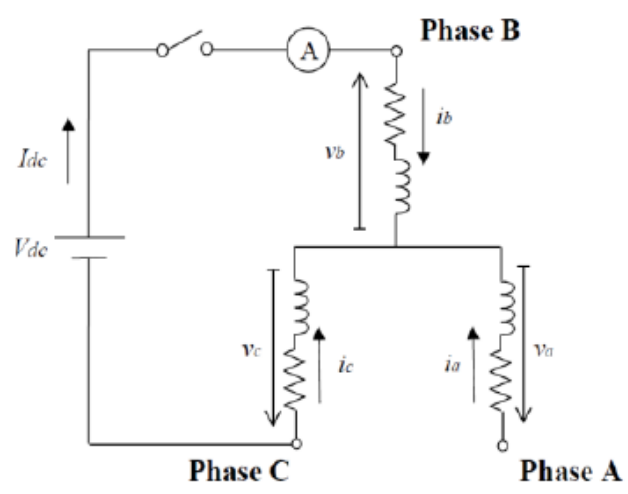

a)

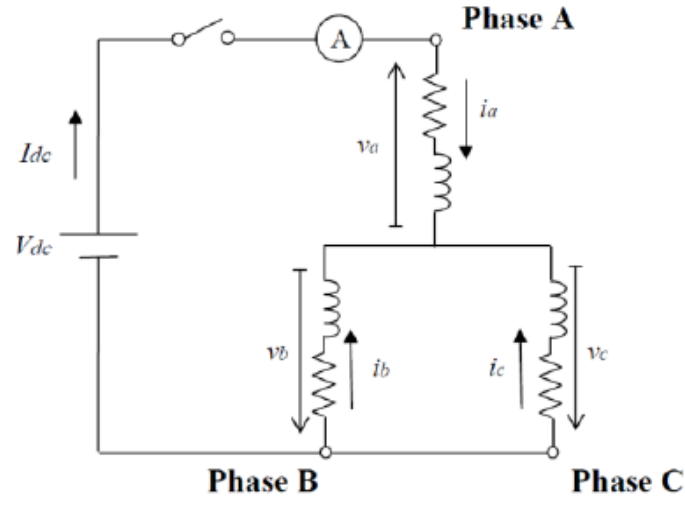

b)

Figure 126: Rotor alignment with d-axis (a) and q-axis (b) [47] 


\section{APPENDIX B}

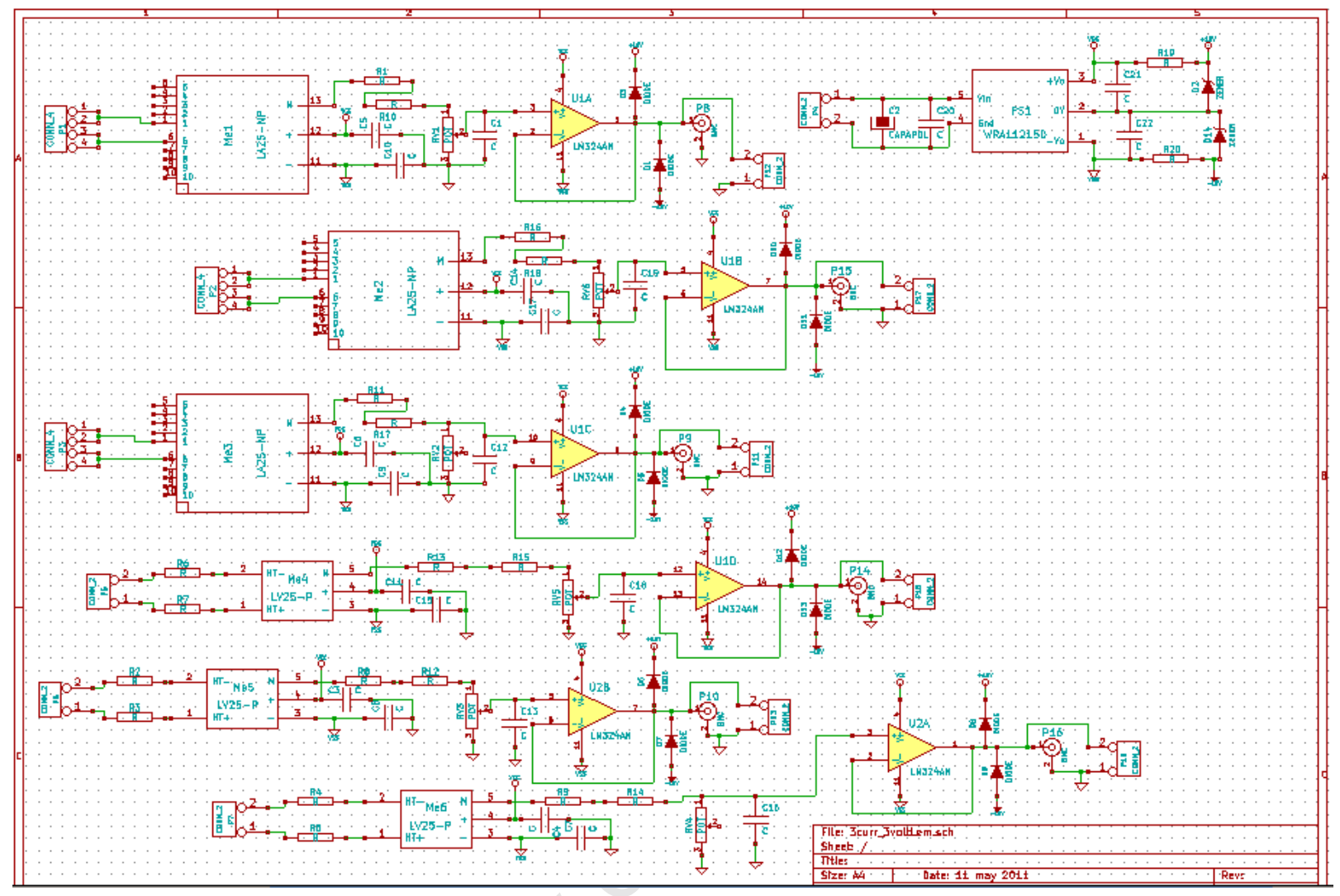

Figure 127: LEM Board Circuit Diagram 


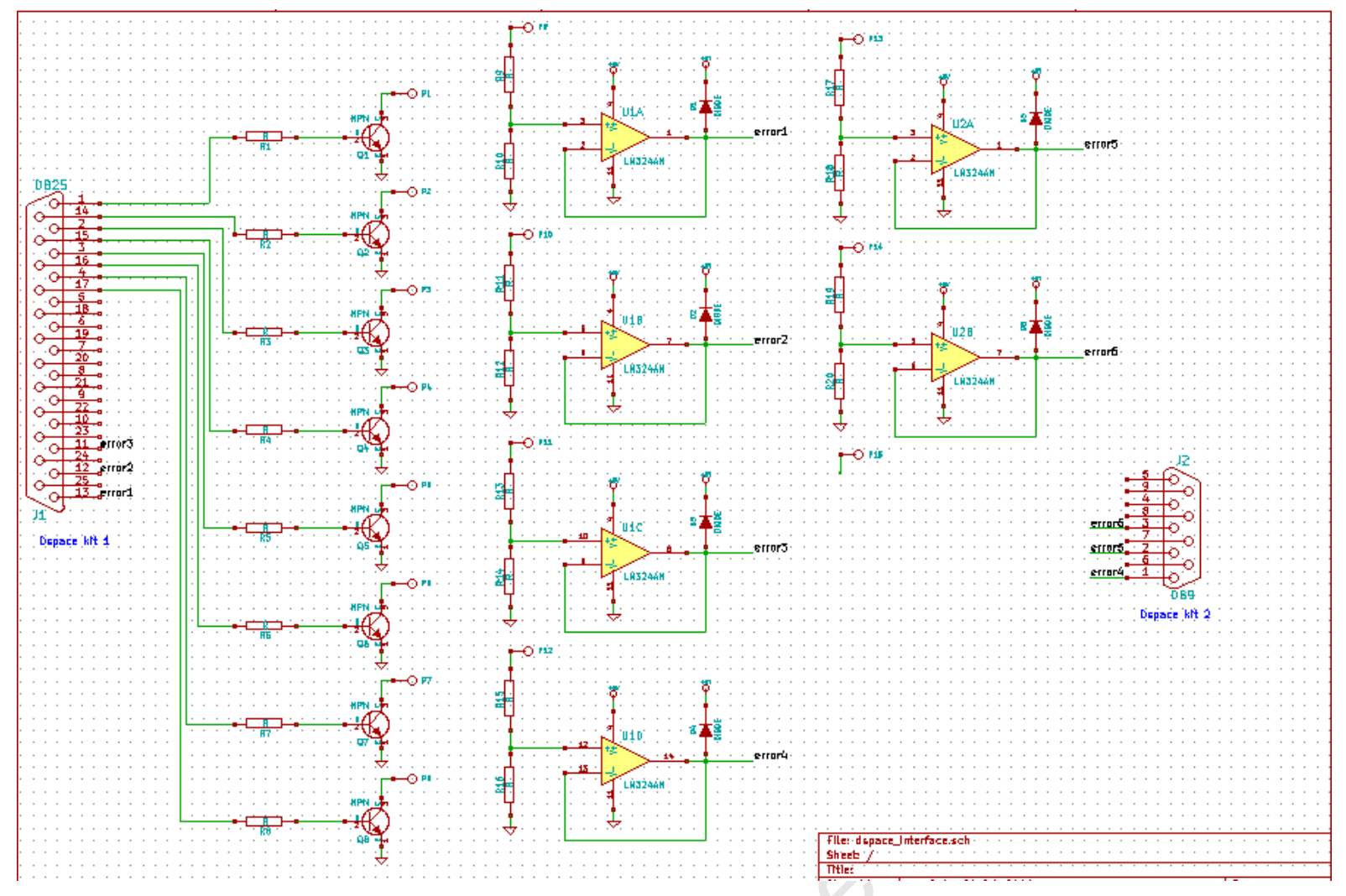

Figure 128: Relay and Error Signal Circuit Board 